\title{
The Application of Spectral Induced Polarization to Determination of Hydraulic Conductivity
}

\author{
By
}

Sheen Joseph

A thesis

submitted to the Victoria University of Wellington

in fulfilment of the requirements for the degree of

Doctor of Philosophy

Victoria University of Wellington 


\section{Abstract}

Spectral Induced Polarization (SIP) is a geophysical technique that measures the frequency dependence of the electrical conductivity of a material. This thesis is an attempt to investigate the potential of using SIP as a proxy to predict the hydraulic conductivity of New Zealand shallow coastal aquifers. SIP measurements were made on sand samples that are typical of New Zealand coastal aquifers with a custom built impedance spectrometer and sample holder allowing the measurement of a phase difference as small a milliradian.

Even though the relaxation time shows a small dependence on pore fluid conductivity, especially at lower pore fluid conductivities, this variation is not serious enough to affect the hydraulic conductivity estimation at the field scale, but could be significant in the investigation of mechanisms that cause polarization in porous media.

Measurements on sieved fractions of sand established that there is an excellent correlation between the Cole-Cole relaxation time constant and grain size. The Cole-Cole relaxation time constant is very sensitive to the grain size distribution. Hydraulic conductivity predictions were attempted using various existing models. While the results are encouraging, it looks like there may not be a single universal model to predict hydraulic conductivity using SIP response.

When a correction term in the form of a multiplication constant is used, all the tested models seem to make very good predictions. But the constants calculated by fitting to the measured data could be applicable only to the type of materials studied. The dependence of the existing models on quantities like counterion diffusion coefficient, electrical formation factor and porosity makes hydraulic conductivity prediction challenging as these quantities are difficult to measure accurately in a field setting. Nevertheless it is concluded that SIP can be successfully applied to study hydraulic conductivity of New Zealand shallow coastal aquifers. 


\section{Acknowledgements}

I would like to express my sincere gratitude to my supervisors, Dr. Malcolm Ingham and Dr. Gideon Gouws for their support and guidance throughout the Ph.D. project. A big thanks to Allan Rennie, Manu Poujaen-Blakiston and Nick Grinter for their help in constructing the sample holders. I am grateful to Peter Coard and Rodney Brown for their support in instrumentation. I thank the School of Geography, Environment and Earth Sciences for permitting me to use the sieve shaker and Jane Chewings for helping me with sieving sand. 


\section{Contents}

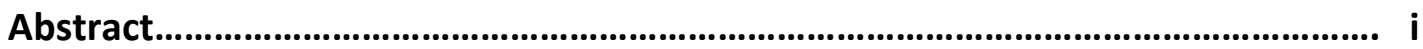

Acknowledgements................................................................................................................ iii

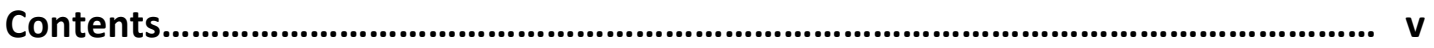

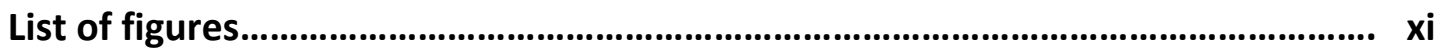

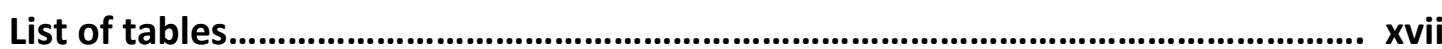

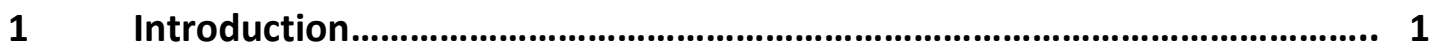

1.1 Geophysical techniques in hydrology.................................................... 3

$2 \quad$ Electrical methods in geophysics................................................................... 7

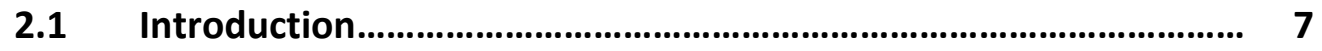

$2.2 \quad$ The self-potential method................................................................. 7

2.3 Direct Current (DC) resistivity methods......................................... 8

2.3.1 Principle of DC resistivity methods..................................... 8

2.3.2 DC resistivity survey configurations..................................... 13

2.3.2.1 Vertical Electrical soundings................................... 13

2.3.2.2 Horizontal Traversing or profiling........................... 14

2.3.3 Electrical resistivity tomography...................................... 14

2.3.4 3D and borehole techniques.............................................. 17

2.3.5 Data Interpretation............................................................... 18

2.3.6 Forward and inverse problem....................................... 20

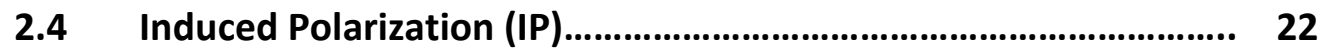

2.4.1 IP measurement systems.............................................. 22 
2.4.1.2 IP in the frequency domain

2.4.1.3 Spectral Induced Polarization (SIP).

2.5 History of SIP

2.6 Conclusion

3 SIP mechanisms and SIP spectra models

3.1 Introduction

3.2 IP mechanisms

3.2.1 Electrode polarization or overvoltage

3.2.2 Membrane or electrolytic polarization.

3.2.3 Maxwell-Wagner polarization.

3.2.4 Polarization of the diffuse layer 36

3.2.5 Polarization of the Stern layer. 38

3.3 The influence of the nature of the medium on SIP mechanism.... 39

3.4 SIP a mechanistic approach....................................................... 41

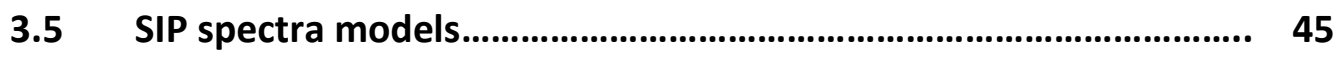

3.5.1 The Debye model........................................................... 45

3.5.2 The Cole-Cole model......................................................... 46

3.5.3 The Pelton model............................................................. 48

3.5.4 The Cole-Davidson model or generalised Cole-Cole model.. 48

3.5.5 The Cole-Cole model with multiple dispersion terms 49

3.5.6 The generalised Cole-Cole model with high frequency capacitive element. 
$4 \quad$ Instrumentation and Experimental Setup.

4.1 Introduction .53

4.2 Operation and the construction of the impedance spectrometer..53

4.2.1 The data acquisition device - NI-USB-6009. .55

4.2.2 The microcontroller - ATTiny84 .56

4.2.3 The signal generators .56

4.2.3.1 The Tektronix AFG 3102 .56

4.2.3.2 The custom made signal generator with direct digital synthesizer. .56

4.2.4 The operational amplifier .58

4.2.5 Electrode selection multiplexors .58

4.2.6 Current control relays .58

4.2.7 The electrodes .59

4.2.8 PC with interface software .59

4.3 Measurement of DC resistance. .60

4.4 Measurement of impedance. .61

4.4.1 Number of frequencies in the SIP spectra .63

4.4.2 The frequency of sampling and the number of samples 63

4.4.3 Testing of impedance spectrometer with an RC circuit........64

4.4.4 Testing of impedance spectrometer with water of various electrical conductivities. 
4.5 Hydraulic conductivity measurements..........................................68

4.5.1 The permeameter................................................................69

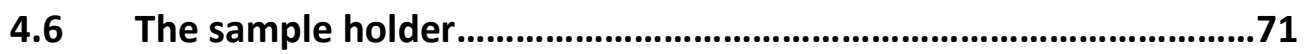

4.6.1 The current and potential electrodes....................................72

4.6.2 Construction of the sample holder........................................73

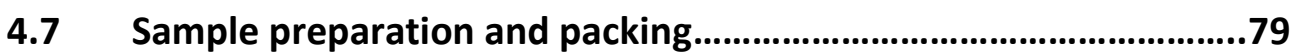

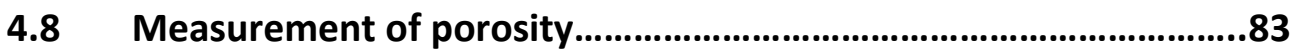

4.9 Determination of electrical formation factor...................................84

4.9.1 Experimental method to determine electrical

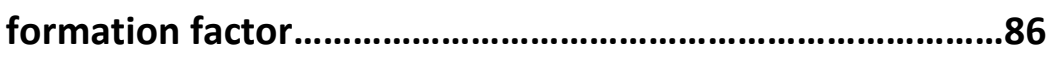

4.9.2 A method for estimating intrinsic formation factor

from a single apparent formation factor..............................86

4.10 Measurement of electrical conductivity of pore fluid....................87

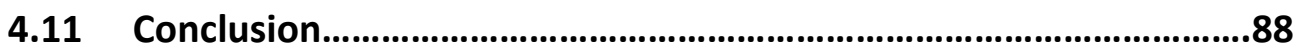

$5 \quad$ Dependence of SIP on pore fluid conductivity.............................................89

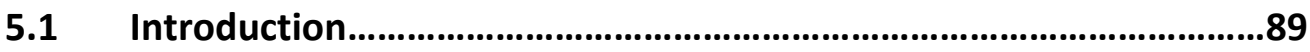

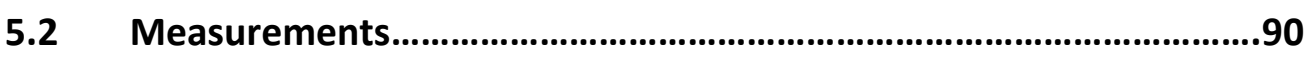

5.3 Cole-Cole modelling...................................................................94

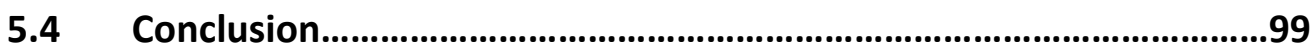

$6 \quad$ Variation of hydraulic conductivity with grain size.......................................101

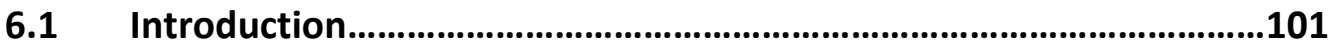

6.2 Permeability from grain size distribution.............................................101 
6.3 SIP measurements as a predictor of permeability

6.4 Sample preparation and measurement..............................................110

6.5 SIP and hydraulic conductivity measurements

on sieved sands

6.6 Correction to the formation factor for Series 1

Samples.

6.7 Relationship between $\tau_{C C}$ and grain size.

6.8 SIP and hydraulic conductivity measurements on

mixtures of grain sizes

6.9 Prediction of permeability from SIP for New Zealand

coastal aquifers

6.10 Dependence of SIP on pore fluid conductivity

- further observations.

6.11 Conclusion .134

7 Summary

Appendix 1. .139

Appendix 2 .141

References 


\section{List of figures}

Figure 2.1: Flow of current from a point source and resulting potential distribution..9

Figure 2.2: A conventional four electrode array 10

Figure 2.3: Current streamlines and equipotentials in a homogenous half space....10

Figure 2.4: Typical current and idealized voltage waveforms for field

DC resistivity Surveys .12

Figure 2.5: Measurement of time-domain induced polarization .13

Figure 2.6: Example of Schlumberger array sounding curve for VES survey. .14

Figure 2.7: Example of horizontal traversing

Figure 2.8: The arrangement of electrode for 2-D electrical survey and sequence of measurements to build up a pseudosection .15

Figure 2.9: The use of roll-along method to extend the area covered by an ERT array 16

Figure 2.10: An example of Wenner pseudosection.

Figure 2.11: Measurement of ERT apparent resistivity pseudosection, the calculated apparent pseudosection, and the inverse model 18

Figure 2.12: Using the roll-along method to survey a 10 by 10 grid with 50 electrode syatem .19

Figure 2.13: Two layer apparent master curves for the Wenner array......... .20

Figure 2.14: Definition of electrical forward and inverse problems .21

Figure 2.15: Transient decay of electric field strength in a rock sample and transient decay of voltage in an $\mathrm{R}-\mathrm{C}$ circuit.

Figure 2.16: Current and voltage waveforms in SIP measurements showing a phase difference.

Figure 3.1: Schematic of the geometry of current passages in sandy-clay sediments .32

Figure 3.2: Various types of pores in rocks .33

Figure 3.3: Illustration of elements of electrode polarization. .34 
Figure 3.4: Various types of fluid filled pores in a rock

Figure 3.5: Development of membrane polarization due to constriction within a channel and that due to presence of clay particles. .37

Figure 3.6: Formation of Stern and diffuse layer around a mineral grain .38

Figure 3.7: Sketch of membrane polarization of a pore sandwiched between two grains 39

Figure 3.8: Three types of porous materials characterised by different polarization mechanisms

Figure 3.9: Phase angle spectra fitted with a Cole-Cole model that has two relaxation terms. .50

Figure 4.1: Block diagram of the impedance spectrometer used for SIP measurements .54

Figure 4.2: Photograph of the impedance spectrometer used for SIP

Measurements. .55

Figure 4.3: Tektronix AFG 3102 used initially for generating low frequency signals .57

Figure 4.4: A screen shot of the front panel of the LabVIEW control software. .60

Figure 4.5: Typical IP effect displayed by a sand sample .61

Figure 4.6: Circuit diagram for the R-C Circuit used to check accuracy of measurement of phase by the impedance spectrometer. .65

Figure 4.7: Calculated and measured phase for the R-C circuit for measurements made over 1 cycle at each frequency .65

Figure 4.8: Calculated and measured phase for the R-C circuit for measurements made over 2 cycles at each frequency... 66

Figure 4.9: Calculated and measured phase for the R-C circuit for measurements made over 3 cycle at each frequency.

Figure 4.10: Measurements of phase made on water samples of different conductivities.

Figure 4.11: SIP phase measurements made on pure silica sand .68

Figure 4.12: Photograph for the set up for permeability measurement .70 
Figure 4.13: Schematic of the sample holder and measurement system used for SIP Measurements.

Figure 4.14: PVC pipe cut into three equal pieces that forms the three sections of the sample holder

Figure 4.15: The ring electrodes made up of stainless steel rod that are used as potential electrodes

Figure 4.16: The closing lids made up of end caps.

Figure 4.17: The current electrode made by soldering brass mesh onto brass electrode .75

Figure 4.18: The mesh electrode placed inside the end cap. .76

Figure 4.19: The straight connector with potential electrode joined to one of the PVC pipes. .76

Figure 4.20: Straight connectors inserted onto both sides of the PVC pipe that forms the middle part of the sample holder

Figure 4.21: The two pieces of PVC pipes connected at the ends of the straight connector.

Figure 4.22: The completed sample holder. .78

Figure 4.23: Map of north island of New Zealand showing locations of sample collection

Figure 4.24: The comparative measurements on BAM reference material over three years at six different German institutions

Figure 4.25: Variation of apparent formation factor with fluid specific conductance of the well sorted, clay-free, medium grain sand .85

Figure 4.26: Method of calculation of intrinsic formation factor from the plot of conductivity of pore water against conductivity of the sample.....

Figure 4.27: The YSI model 3100 conductivity meter used for electrical conductivity measurements

Figure 5.1: Schematic measured grain size distribution for the natural sand used in Samples 1 and 2 .90

Figure 5.2: Conductivity magnitude and phase of measurements on sample 1 for different fluid conductivities .92 
Figure 5.3: Conductivity magnitude and phase of measurements

on sample 2 for different fluid conductivities

Figure 5.4: Variation of Cole-Cole parameter DC resistivity $\left(\sigma_{0}\right)$

with fluid conductivity .96

Figure 5.5: Variation of Cole-Cole parameter relaxation time $\left(\tau_{C C}\right)$ with fluid conductivity. .96

Figure 5.6: Variation of Cole-Cole parameter chargeability (m)

with fluid conductivity.

Figure 5.7: Variation of Cole-Cole exponent (c) with fluid conductivity......

Figure 5.8: Variation with fluid conductivity of $\left(\sigma_{0}\right)$ of the in-phase $\left(\sigma_{\text {surf }}^{\prime}\right)$

and quadrature-phase parts of the surface conductivity

Figure 6.1: Measured permeability plotted against grain size for

Series 1 samples.

Figure 6.2: Measured permeability and permeability predicted using Kozeny-Karman equation plotted against grain size 104

Figure 6.3: Flow chart summarising efforts to predict hydraulic conductivity from petrophysical relationships established for electrical measurements.....105

Figure 6.4: Typical example of SIP phase spectrum showing a peak in the phase of the complex conductivity at low frequency 106.

Figure 6.5: SIP phase measured on sands of specified grain sizes- Series 1 and Series 2

Figure 6.6: Fit of Cole-Cole model to SIP amplitude and phase for $\varphi=1.5$.

Figure 6.7: Measured permeability plotted against predicted permeability

for Series 1 and Series 2

Figure 6.8: Relaxation time derived from Cole-Cole modelling plotted against mesh size for Series 1 and Series 2 .

Figure 6.9: SIP phase spectra measured on mixtures of saturated sands with different proportions of grain sizes

Figure 6.10: Variation in measured porosity, formation factor, Cole-Cole relaxation time and measured permeability for mixtures of sands with different 
proportions of grain sizes. .122

Figure 6.11: Measured permeability plotted against predicted permeability for Series 1, Series 2 and mixtures of grains.

Figure 6.12: Measured permeability plotted against predicted permeability using equations corrected with a multiplicative constant for Series 1, Series 2 and mixtures of grains. .126

Figure 6.13: Measured permeability plotted against predicted permeability for equations used by Weller et al., (2015) .128

Figure 6.14: Measured permeability plotted against predicted permeability comparison with data from Koch et al, (2012)

Figure 6.15 Plot of Cole-Cole relaxation time constant with grain size - comparison with data from Koch et al. (2012). 130

Figure 6.16 Measured permeability plotted against permeability predicted by equation used in Weller et al., (2015)- comparison with a data from Weller et al. (2015). .131

Figure 6.17: Cole-Cole relaxation time constant plotted against DC electrical resistivity 132

Figure 6.18: Measured and estimated values of Cole-Cole relaxation time constant plotted against DC electrical resistivity. 


\section{List of Tables}

Table 2.1: Commonly used electrode configurations four electrode electrical Measurements.

Table 3.1: Models, equivalent circuits and complex resistivities

proposed for SIP .46

Table 4.1: Values of current that can be selected in the impedance spectrometer and the corresponding maximum value of load resistance. 61

Table 4.2: The time taken for one complete set of measurement for three

different mumber of frequencies per decade and three different duration of sample collection expressed in terms of the cycle of the respective frequencies.

Table 5.1: Cole-Cole parameters recovered from fitting the measured SIP spectra for samples with different fluid conductivities . .95

Table 6.1: The porosity, formation factor, cementation exponent and measured values of permeability for Series 1 and Series 2 samples.

Table 6.2: Results of Cole-Cole fitting to SIP spectra of Series 1 and

Series 2 samples 114

Table 6.3: Porosity, formation factor, cementation exponent, measured permeability and Cole-Cole relaxation time for mixture of grains .121 


\section{Introduction}

Water is the elixir of life. There is no life without water. The movement of water between land, oceans and the atmosphere, called the hydrologic cycle, ensures the continuous availability of freshwater to all forms of life on land. Water is essential for agriculture and all forms of economic activity. It is so important to us that many early civilizations e.g. Babylonian, Egyptian, Chinese, Mexican and Indus Valley evolved around water sources that supplied freshwater in abundance. The survival of early civilizations depended on their ability to manage water resources. Water is plentiful on our planet but the difficulty is that enough water may not be available at places that are suitable for human inhabitation. For a long time the main source of freshwater was surface water which is available in the form of rivers, lakes and wetlands. But such natural sources alone were never sufficient to supply enough water for increasing populations. Hence ancient civilizations developed elaborate water supply systems like aqueducts and canal systems to harvest surface water. Qanats, series of gently sloping underground tunnels that carry water from a 'mother' well at a higher elevation to low-lying neighbouring areas, were used to access groundwater in southwest Asia, North Africa and the Middle East (Cech, 2003). Like the ancient civilizations we continue the process of managing our water resources through storage, redistribution and regulation to satisfy the need for drinking water, irrigation and flood protection.

Dramatic increase in population over the past two centuries has increased the demand for freshwater. As surface water resources are already utilised to maximum capacity the focus has been on groundwater. This is natural because about $95 \%$ of accessible freshwater exists as groundwater (Chilton, 1992). The term groundwater is used to indicate subsurface water that is present below the water table. It is the only form of freshwater available in many areas. Groundwater has the advantages that it does not require a storage reservoir. Unlike surface water reservoirs it is free from loss caused by evaporation and protected from contaminants by the soil cover. Groundwater has contributed significantly to the growth of irrigated areas benefiting millions of farmers in the past 50 years.

Until recently the dependence on groundwater was limited to shallow wells, springs and mountainside tunnels. The advancement in drilling technology and pumping systems 
have made possible the production of deep wells and low cost submersible pumps. Government subsidies have made the use of groundwater even more attractive for farmers in many countries. Initially aquifers were considered as having a capacity to supply unlimited amounts of water and not much attention was given either to the rate of usage or to protecting them. Over time the intensive use of aquifers has led to either depletion or degradation in their quality. This has made people realise that groundwater cannot be taken for granted.

The increased use of groundwater for both irrigation and domestic purposes has, in many cases, led to abstraction of groundwater at a rate greater than its recharge. This is referred as 'intensive use of groundwater'. The intensive use of groundwater can cause a multitude of negative effects which are threatening to the prosperity generated by the use of groundwater. The first negative effect is that the intensive use of groundwater may cause a decrease in the groundwater levels. This makes extraction more costly; sometimes levels can be so low that the existing wells are rendered useless. As the pressure drops there is a decrease in spring discharge, river base flow and the surface area of wetlands (Custodio, 2002). Intensive use of groundwater can also cause geological hazards like land subsidence, earth fissures and surface faults, sinkholes, seawater intrusion and some less common hazards such as earthquakes and landslides (Angelos, 2011).

Contamination of groundwater by various anthropogenic activities is another threat to aquifers, in addition to the over use of groundwater. The sources of these contaminants may be domestic waste, sewage, industrial waste and effluents like hydrocarbon products, fertilisers and pesticides, saline water intrusion etc. Once the contaminants get into the subsurface they keep spreading affecting the quality of groundwater and the ecosystem. So depletion and contamination of aquifers are definitely important challenges ahead. Decontamination and remediation is very difficult and expensive.

Quality freshwater is a necessity for domestic, agricultural and industrial purposes. Human consumption of freshwater is increasing. This puts greater stress on freshwater resources. The increasing population and depleting freshwater resources provide a formidable challenge for governments. This calls for management of groundwater resources in terms of identifying new locations for high yielding aquifers, deciding the sustainable usage pattern of an aquifer, and enhancing its recharge or artificial recharge. 
Steps have to be taken for the prevention of contamination from various effluents both industrial and domestic. Governments have the responsibility to come up with necessary water management policy and guidelines.

\subsection{Geophysical techniques in hydrology}

Sustainable management of groundwater sources demands a good understanding of hydrological systems and the transport properties of the shallow subsurface of the earth. We need tools to characterize, monitor and investigate hydrogeological parameters and processes. This calls for new and improved hydrogeological characterisation and monitoring methods. The emerging discipline of hydrogeophysics has the aim of applying geophysical methods for mapping subsurface features, estimating properties, and monitoring processes that are important to hydrological studies such as those associated with water resources, contaminant transport, and ecological and climate investigations (Rubin and Hubbard, 2005).

The extent and resolution of the subsurface structure that is required varies according to the type of application, and may vary from a global to a laboratory scale. In particular, detailed high resolution data are required for contamination-related studies. Traditionally, various hydrogeological methods like bore holes etc. have been used for the study of the subsurface. But invasive approaches like this have many drawbacks. It is well known that there is extensive heterogeneity in the distribution of transport properties both vertically and horizontally. There is no way we can be certain that the properties that we estimate by invasive methods represent other than a local picture of the subsurface. More than that, an invasive method does not guarantee an accurate measurement as it disturbs the medium during the process of drilling. Another factor is that invasive methods are costly and time consuming and not practical at many locations because of terrain features and inaccessibility for vehicles.

Geophysical methods on the other hand are either surface methods or require only a few bore holes to cover a large area. They can give high resolution information of hydrogeological properties though they can be time consuming and labour intensive and hence may be more expensive. But the biggest challenge or drawback of geophysical methods is that there is not necessarily a direct relationship between the measured physical properties and the transport properties of the medium. Although the measured 
physical properties can be related to transport properties through some petrophysical relationship, in many cases there is no clarity on how exactly the measured physical properties are related to the transport properties. What also makes the application of geophysical methods more challenging is that the geophysical response of the subsurface is not unique i.e. different transport properties can lead to the same geophysical response. This is especially so for geoelectrical methods. Geoelectrical responses are controlled by a combination of factors such as pore fluid chemistry, temperature, saturation, the presence of metallic minerals etc.

Even with all these challenges in the past two decades there have been tremendous improvements in the application of geophysical methods to subsurface transport property estimation using electrical, electromagnetic, magnetic, ground penetrating radar (GPR), nuclear magnetic resonance (NMR) and seismic methods. This has been accelerated by the advancements in data acquisition and data processing hardware and software. One important concept that has been developed is combining data collected from various geophysical and hydrogeological methods to improve estimates. While hydrogeological methods provide a direct high resolution measurement, geophysical methods gather data over wide area with a decreasing resolution. Hydrogeological data act as some sort of constraint in modelling the transport properties using geophysical methods.

The aim of this PhD study is to test the viability of using Spectral Induced Polarization (SIP) measurements for the determination of hydraulic conductivity, with the specific aim of applying SIP technique to study the hydraulic properties of shallow coastal aquifers of New Zealand. SIP laboratory measurements were undertaken as a precursor to field studies. The study began with adapting a custom-made impedance spectrometer to make high resolution SIP measurements. This was followed by developing the control software that performs data acquisition and sophisticated data analysis and forms first part of this study. SIP Measurements were carried out on samples that are typical of New Zealand coastal aquifers.

As the pore fluid electrical conductivity is a factor that varies considerably in the field setting, investigations were carried out that explore the influence of pore fluid electrical conductivity on SIP spectra. SIP measurements on very well sieved sand fractions were used to study the relationship between the Cole-Cole relaxation time, a parameter 
recovered from SIP response, and the grain size and hydraulic conductivity which forms the basis on which SIP is used to predict permeability. Further measurements on samples made up of a mixture of two different grain size were helpful in proving the sensitivity of Cole-Cole relaxation time to grain sizes distribution. With these new data, various models were tested for their ability to predict hydraulic conductivity.

The structure of the thesis is as follows. The first chapter contains the introduction and the role of hydrogeophysics in groundwater studies. The second chapter is on the electrical methods in geophysics. It goes on to look at the history of electrical methods in geophysics and briefly explains the fundamentals and advancements in the technique over the past century. In the third chapter the phenomenon of Spectral Induced Polarization (SIP) is discussed in detail. It covers the different mechanisms that possibly give an SIP response, and a mechanistic model that explains the origin of the SIP response in unconsolidated media. The fourth chapter gives the details of the instrumentation and measurement procedures adopted for this project. It consists of the design and operational details of the impedance spectrometer and the permeameter set up. It also describes the measurement of the electrical formation factor, porosity, electrical conductivity of pore water, and sample preparation methods. The fifth chapter presents the results of the investigation of pore water electrical conductivity on the SIP parameters. The sixth deals with the relationship between the SIP parameters, grain size and the hydraulic conductivity. This chapter looks at various models used to predict hydraulic conductivity from SIP measurements. The summary is presented in chapter seven. 


\section{Electrical methods in geophysics}

\subsection{Introduction}

Electrical prospecting involves the detection of surface effects produced by electric current flow in the ground [Telford et al., 1976]. The use of electricity to study the subsurface began in 1830 with the work of R.W. Fox in the mines of Cornwall, UK [Fox, 1830]. He studied the natural electric currents associated with sulphide ore deposits. Many scientists followed up the work of Fox and contributed to the development of electrical prospecting (see Van Nostrand and Cook, 1966 for the detailed history of early developments in electrical prospecting). The enormous variation of electrical properties across different geological materials has contributed to the development of a wide variety of electrical methods. Rust Jr. (1938) summarises the developments in the application of electrical methods in geophysics in the first 100 years. The electrical methods commonly used in hydrogeophysics include electromagnetic induction (EMI), ground penetrating radar (GPR), time-domain reflectometry (TDR), self-potential method (SP), electrical resistivity(ER), induced polarization (IP) and spectral induced polarization (SIP). The last four techniques are explained here as they have many things in common and have the advantage that they are non-intrusive, or minimally intrusive, making them especially suitable for hydrogeophysical applications.

\subsection{The self-potential method}

The self-potential method is the oldest (about 185 years) of the electrical methods. The self-potential method is a passive method i.e. unlike the other three electrical methods it does not require any external current source. The self-potential method involves measuring the electrical potential distribution with respect to a reference electrode and then creating a map that describes the distribution of the potential difference over the area of interest. The manifestation of this potential difference between the reference electrode and the electrode at a given location is caused by the existence of electric current in the ground. There are a number of sources for these currents. These include the formation of 'geobatteries', streaming current due to flow of water, and the diffusion currents associated with gradients in the chemical potential of charge carriers (Revil et al., 2012b). Self-potential mapping helps to locate areas of anomalous conductivity and groundwater flow. Hence this method has been used widely to locate 
mineral deposits. It is the simplest of the electrical methods, but the presence of noise, such as telluric currents from electromagnetic induction by the geomagnetic field, makes it difficult to interpret. Details about the theory and applications of the selfpotential method to hydrological problems can be found in Revil et al. (2006).

\subsection{Direct Current (DC) resistivity methods}

DC electric resistivity is a method by which subsurface electrical resistivity is measured using an array of electrodes normally placed on the surface of the ground. Initial surveys were to check for a variation in electrical resistivity which might indicate the presence of materials that have a higher conductivity because of the presence of metallic ores, the presence of water, or an increased concentration of salts in the water. The search for mineral deposits using electrical methods was in place as early as 1883. Two important personalities in the use of electricity for prospecting were Frank Wenner from United States and Conrad Schlumberger from France who worked independently during the same period around 1912. As there is a lot in common, such as survey configurations, between DC resistivity methods and Spectral Induced Polarization methods it is useful to explore the principles of DC electric resistivity methods in detail.

\subsubsection{Principle of DC resistivity methods}

In the DC resistivity method an electric field is set up in the ground using an external source of current. This external current causes an electric potential distribution in the ground. For a 3-D isotropic electrical conductivity distribution $\sigma(\boldsymbol{r})$ the electric potential $V(\boldsymbol{r})$ at a point due to a single current electrode idealized as point source at the origin with strength $I$ is defined by the Poisson equation (Binley and Kemna, 2005).

$\nabla \cdot(\sigma \nabla V)=-I \delta(\boldsymbol{r})$

subject to condition

$\frac{\partial V}{\partial n}=0$

at the ground surface and condition

$V=0$ 
at other infinite boundaries; with $\delta$ the Dirac delta function and $n$ the outward normal. A single current source as explained above creates an electric potential distribution as shown in Figure 2.1. The electric potential at any point is given by

$V=\frac{\rho I}{2 \pi r}$

where $\rho$ is the resistivity and $r$ the distance from the electrode. Usually in a resistivity survey the current is injected into the ground using two electrodes (called current electrodes C1 and C2) and the potential is measured using two other electrodes (called potential electrodes $\mathrm{P} 1$ and $\mathrm{P} 2$ ) as shown in Figure 2.2. Such an array of current sources produces a potential distribution as shown in Figure 2.3. The blue dotted lines in Figure 2.3 indicate the equipotentials. The potential difference between the electrodes P1 and P2 as shown in Figure 2.2 can be calculated using equation (2.4) as

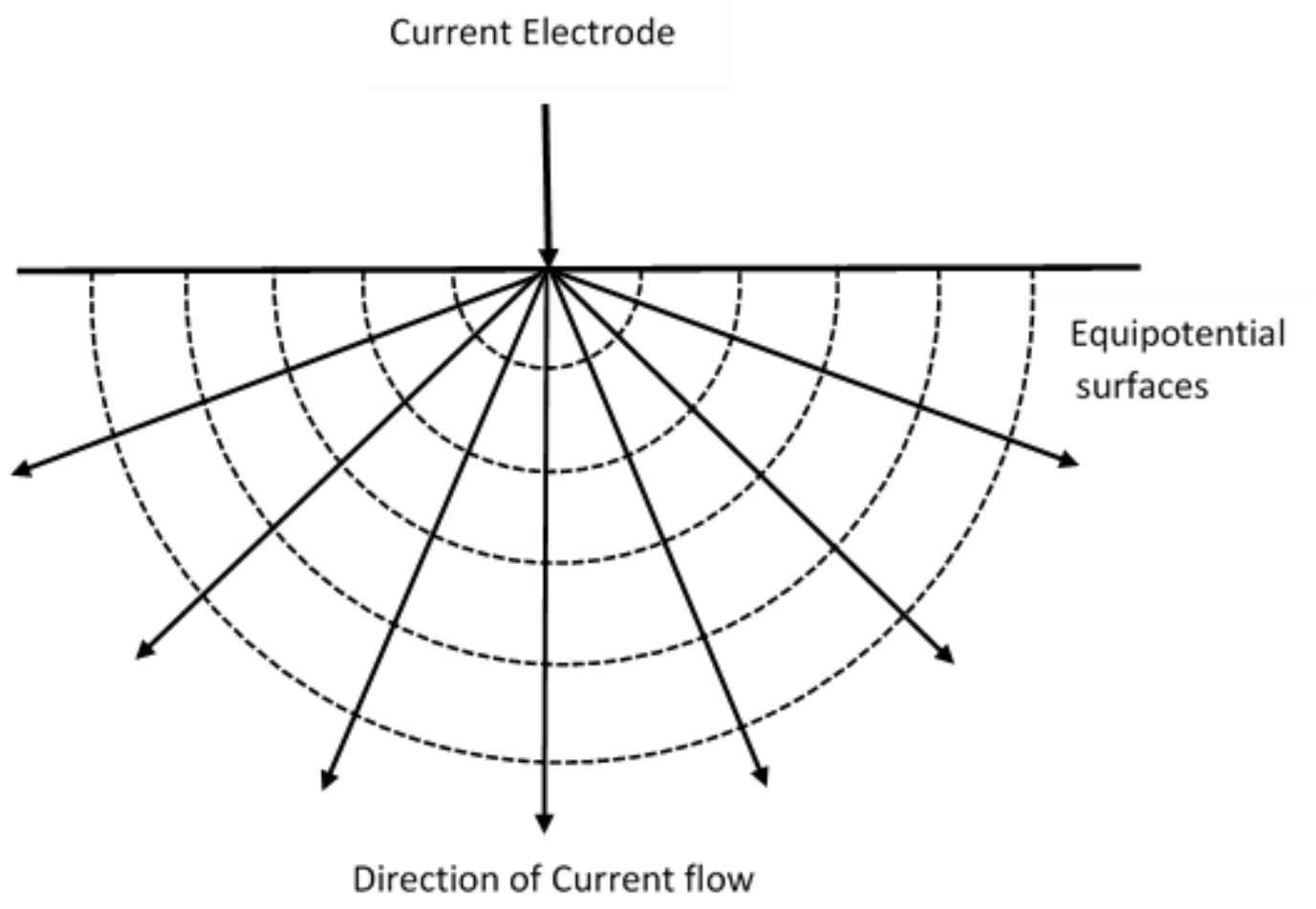

Figure 2.1: The flow of current into a homogeneous half-space from a point current source and the resulting potential distribution. 


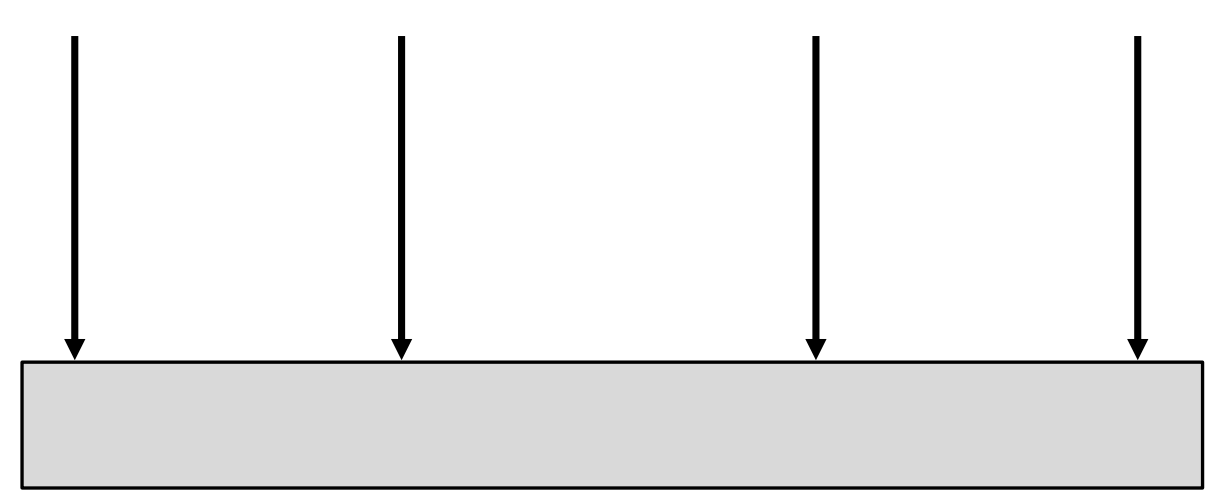

Figure 2.2: A conventional four-electrode array.

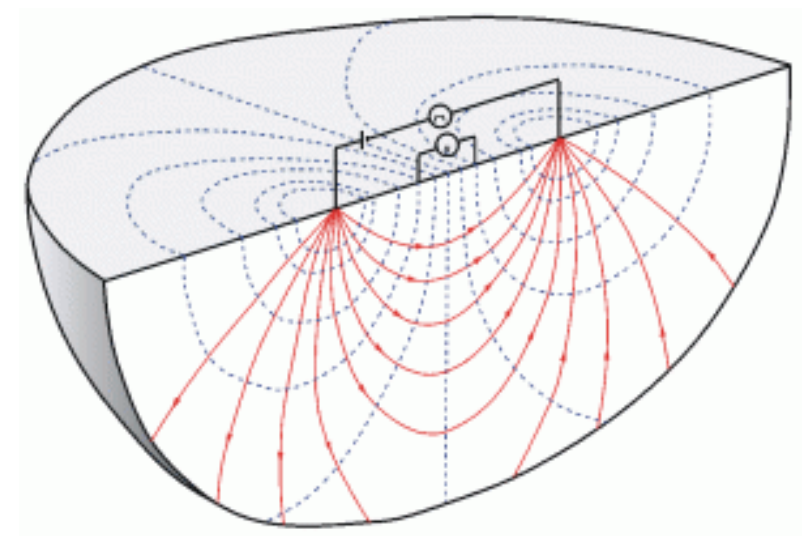

Figure 2.3: Current streamlines (red) and equipotentials (blue) in a homogenous half space. (http://www.tricon-online.de/html/body_geoelektrik.html)

$\Delta V=\frac{\rho I}{2 \pi}\left(\frac{1}{r_{C 1 P 1}}-\frac{1}{r_{C 2 P 1}}-\frac{1}{r_{C 1 P 2}}+\frac{1}{r_{C 2 P 2}}\right)$

In the case of a uniform resistivity distribution the resistivity of the homogenous half space can be written as

$\rho=k \frac{\Delta V}{I}$

where $k=\frac{2 \pi}{\left(\frac{1}{r_{C 1 P 1}}-\frac{1}{r_{C 2 P 1}}-\frac{1}{r_{C 1 P 2}}+\frac{1}{r_{C 2 P 2}}\right)}$

The parameter $k$ is called the geometric factor or configuration factor; the value of which depends upon the type of electrode array. A list of commonly used four electrode arrays and the respective geometric factors is given in Table 2.1. 


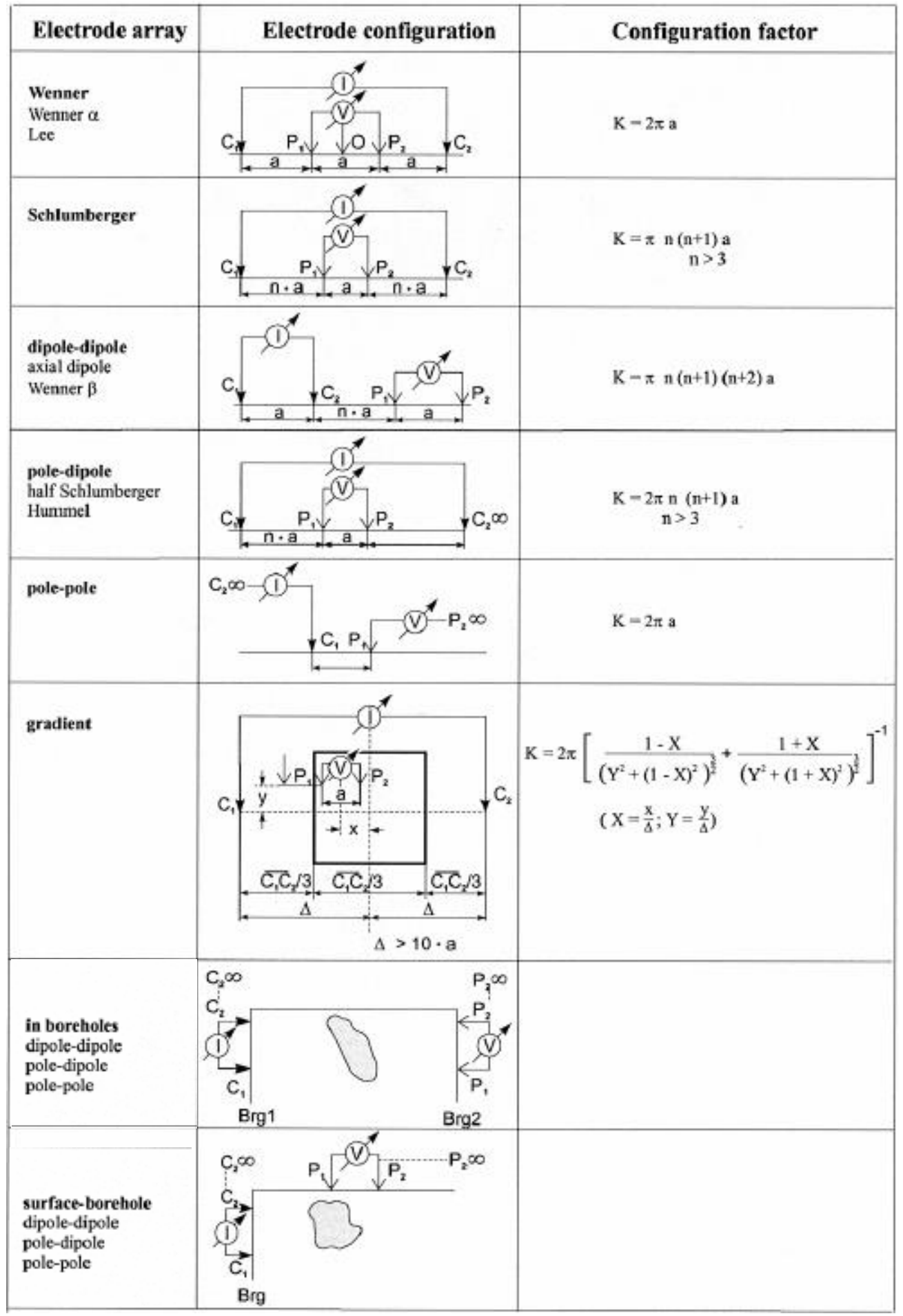

Table 2.1: Electrode configurations. (Seidel and Lange, 2007).

Each of these configurations has its advantages and disadvantages with regard to depth of investigation, resolution of horizontal and vertical structures, and sensitivity to lateral changes in resistivity and inhomogeneity (Seidel and Lange, 2007). A suitable configuration is selected based on the requirements of the survey. Further details of 
electrode configurations can be found in (Szalai and Szarka, 2008), (Whiteley, 1973) and (Ward, 1990).

In all practical cases the surface under investigation will not have a uniform resistivity distribution. Hence in the case of an inhomogeneous resistivity distribution the resistivity as calculated by equation (2.6) is not a true resistivity i.e. it does not represent the resistivity of the subsurface under investigation, nor the resistivity of any layer/portion of the subsurface. It is not even some sort of average of the true resistivities. The resistivity as calculated in a DC resistivity survey is called the "apparent" resistivity. The apparent resistivity is the resistivity of a homogenous ground that will give the same resistivity value for the same electrode arrangement. True resistivity values can be estimated from apparent resistivity values.

DC resistivity instruments usually use a switched square wave current signal like the one showed in Figure 2.4. This helps to reduce the polarization at the electrodes. An ideal measured voltage waveform should be similar to the applied current waveform. But in reality the measured voltage wave differs from the current signal because of background self potential and the capacitance of the ground as shown in Figure 2.4 and Figure 2.5 respectively
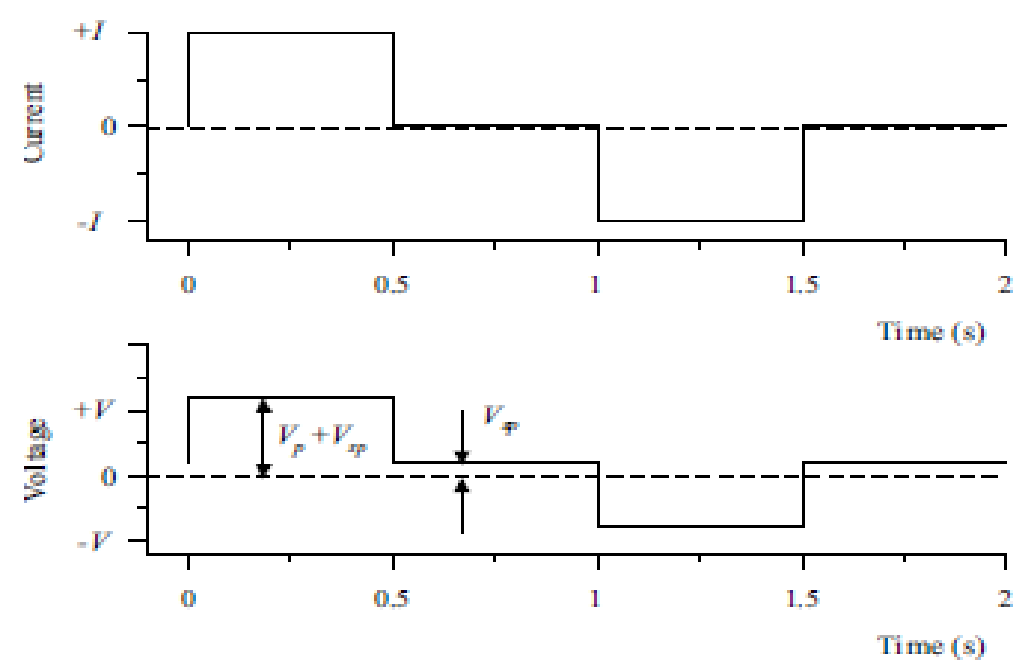

Figure 2.4: Typical current and idealized voltage waveforms for field DC resistivity surveys. $V_{p}$ is the primary voltage, $V_{s p}$ is the observed self potential voltage. (Binley and Kemna, 2005). 


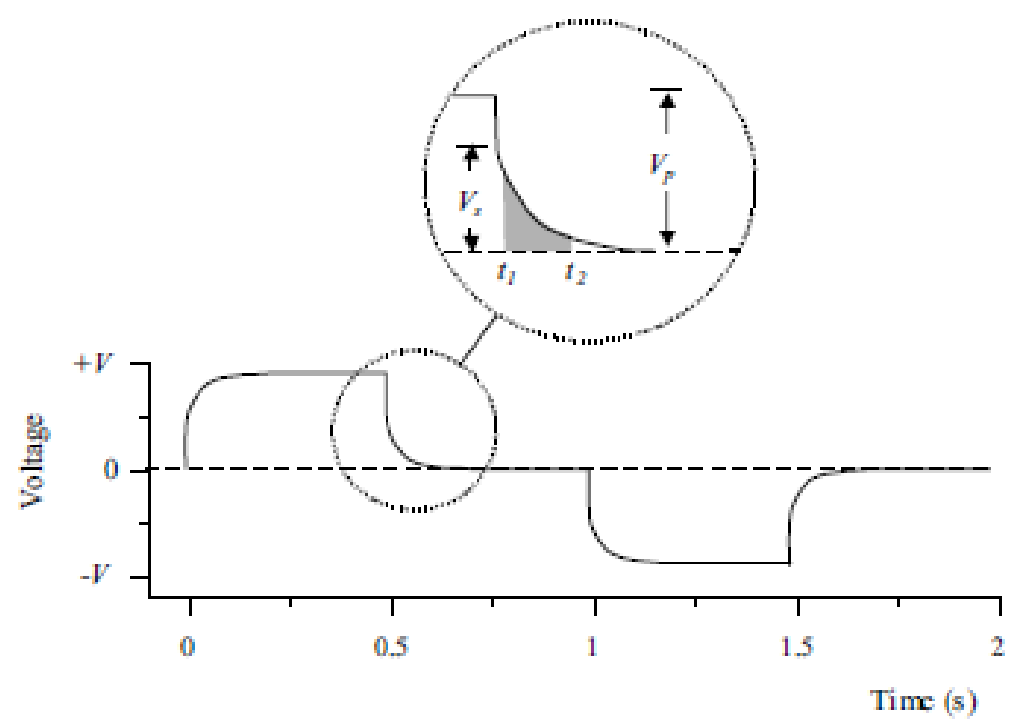

Figure 2.5: Measurement of time-domain induced polarization. (Binley and Kemna, 2005).

\subsubsection{DC resistivity survey configurations}

The method of conducting a DC resistivity survey is not unique. The survey configurations differ from each other in the manner in which the electrode placements are changed during the measurements. These different types, of electrode placement methods are called DC resistivity survey configurations, or modes of deployment. Each type of survey configuration provides resistivity data about a different region of the subsurface under study. The choice of survey type depends on various factors like the existing information about the geology, time frame, equipment used etc. The survey configurations explained below can be used for DC resistivity, IP and SIP methods.

\subsubsection{Vertical electrical soundings}

A vertical electrical sounding (VES) is carried out by making a series of resistivity measurements such that while electrode separation is increased in each subsequent measurement the centre of the electrode array remains at the same point. This means that a VES effectively measures the apparent resistivity at different depths. This method is best suited when the subsurface can be considered as a series of horizontal layers, each with uniform but different resistivity. The variation of apparent resistivity with depth is a function of the resistivity and thickness of each layer. VES can provide a good picture of the vertical distribution of resistivity. The result of a VES is vertical sounding curve which is a plot of the measured apparent resistivity against a measure of depth of 
penetration of electrical current. Figure 2.6 shows a sounding curve and the interpreted resistivity values for a Schlumberger array sounding.
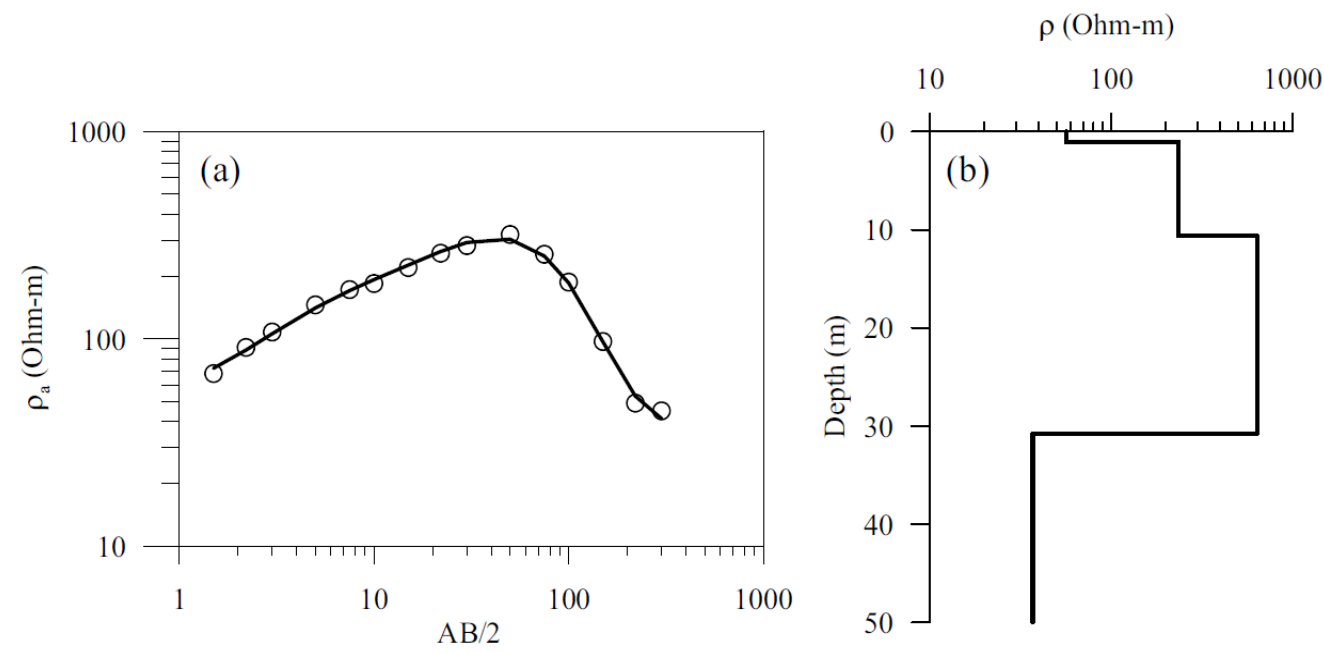

Figure 2.6: (a) Example of a Schlumberger array sounding curve from a VES survey. Circles indicate the measured data and the solid line shows the modelled curve. $A B / 2$ (in $m$ ) is the half distance between the current electrodes. (b) Interpreted model of resistivity as a function of depth at a single location. (Binley and Kemna, 2005).

\subsubsection{Horizontal traversing or profiling}

Horizontal Traversing or Constant Separation Traversing (CST), also called surface profiling, is a technique for detecting lateral variations in resistivity along a straight line. The measurements involve moving the electrode array along a straight line for consecutive measurements while maintaining constant electrode separations. This provides the resistivity at a particular depth along the profile. The depth at which the resistivity is measured depends on the electrode separation. Figure 2.7 shows a constant separation traverse using a Wenner array with $10 \mathrm{~m}$ electrode spacing over a clay filled feature(position arrowed) in limestone [Reynolds, 2011].

\subsubsection{Electrical resistivity tomography}

Electrical Resistivity Tomography (ERT) which is also known as Subsurface Imaging (SSI) effectively combines both vertical electrical sounding and horizontal traversing. ERT measurements are made with modern field systems that use multicore cables and computer/microprocessor controlled 'smart' electrodes. Multicore cables come with 25 


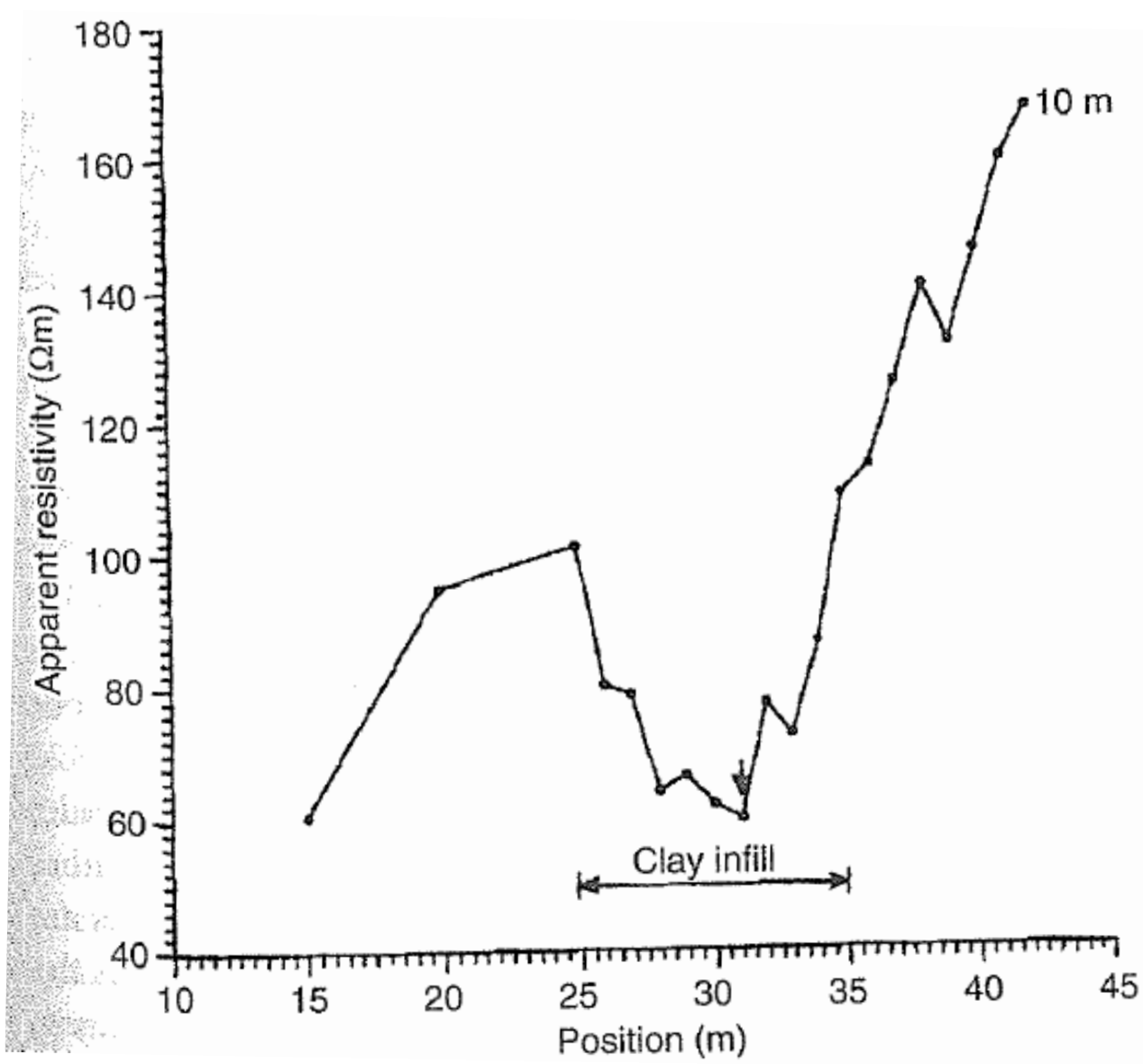

Figure 2.7: Example of horizontal traversing (Reynolds,2011).

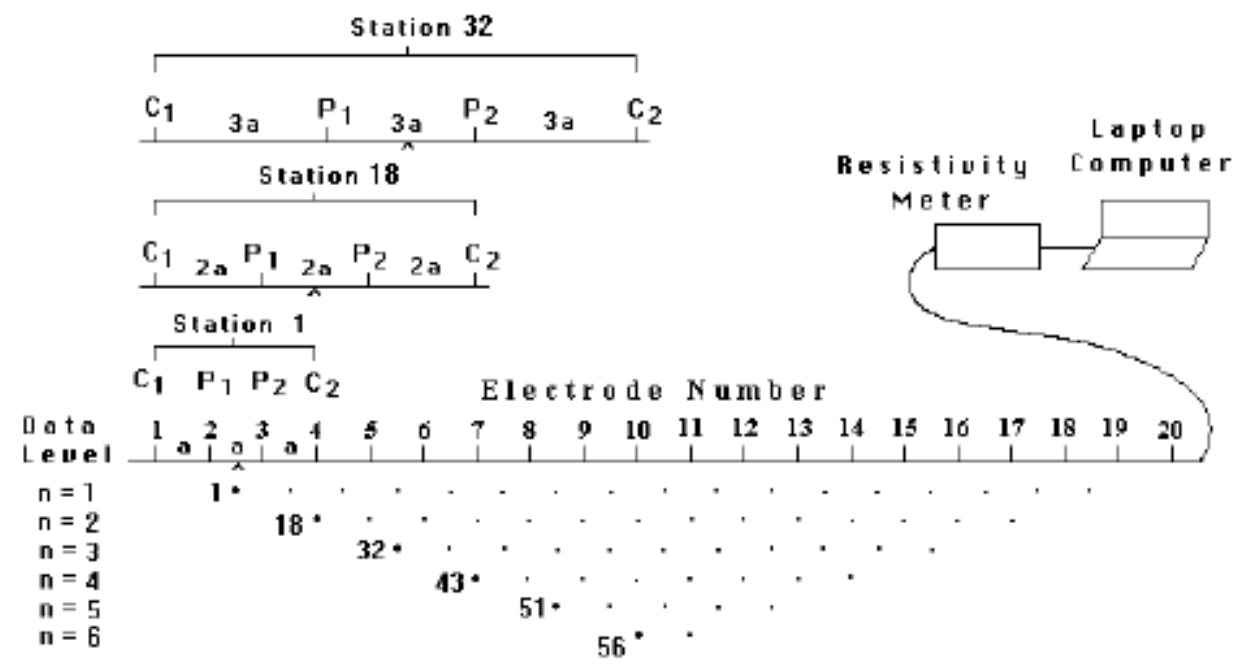

Figure 2.8: The arrangement of electrodes for a 2-D electrical survey and the sequence of measurements used to build up a pseudosection (Loke, 2004). 
or more takeouts for connecting electrodes spaced at constant separation like $5 \mathrm{~m}$ or $10 \mathrm{~m}$ and may also have the provision to connect a number of rolls one after the other. A four electrode resistivity measurement is possible with any of these 'smart' electrodes as a current electrode or potential electrode. Figure 2.8 shows the sequence of measurements to produce an ERT profile using a Wenner array of electrodes.

At first all possible measurements are made with an electrode separation $a$. The next set of measurements will be for all possible combination of electrodes for a separation of $2 a$. In the successive steps the electrode separation is increased as $3 a, 4 a, 5 a$ and so on. This process is repeated until the electrode separation reaches the maximum possible value. After completing all possible measurements with the available multicore cable rolls the measurement can be extended in the horizontal direction by the rollalong method. In the roll-along method the first roll of cable is removed and connected at the other end of the cables as shown in Figure 2.9.

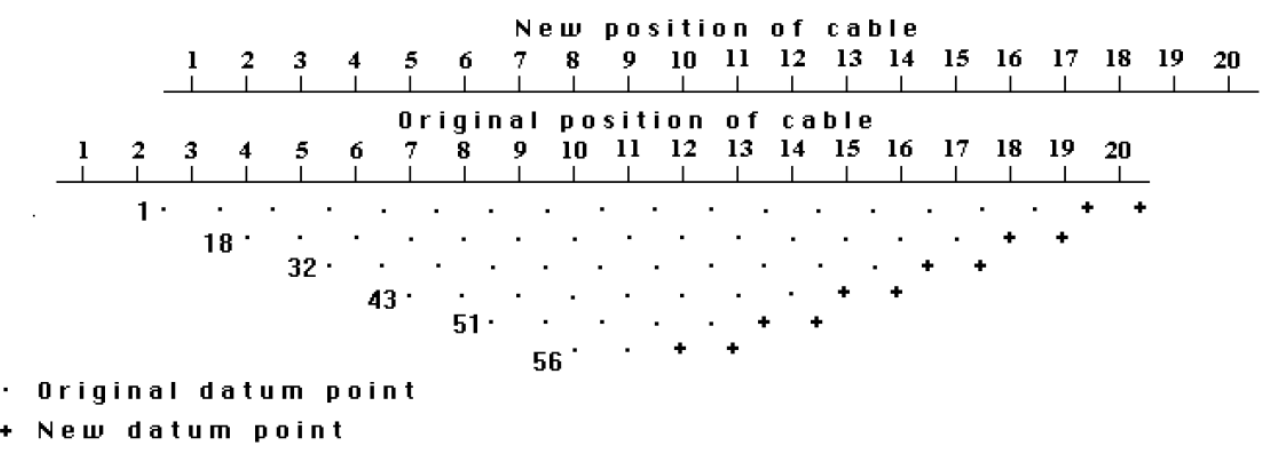

Figure 2.9: The use of roll-along method to extend the area covered by an ERT survey (Loke, 2004).

The measurements are then repeated as explained previously. Using these resistivity measurements a pseudosection image is generated by assigning certain locations for each of the resistivity measurements. The horizontal position of resistivity measurement on the pseudosection is selected as the midpoint of the electrodes and the vertical position is taken as a distance proportional to the separation between the electrodes (Loke, 2004). A contoured pseudosection like the one in Figure 2.10 provides some general information about the subsurface even though it is a distorted representation of true resistivity and true depth. 
The real power of ERT comes from the fact that by using a form of processing known as inversion the pseudosection can be converted into an image that represents the true depth and true resistivities (Barker, 2007). Figure 2.11 shows the images of a pseudosection, the respective computer modelled pseudosection and the ERT image produced after inversion.

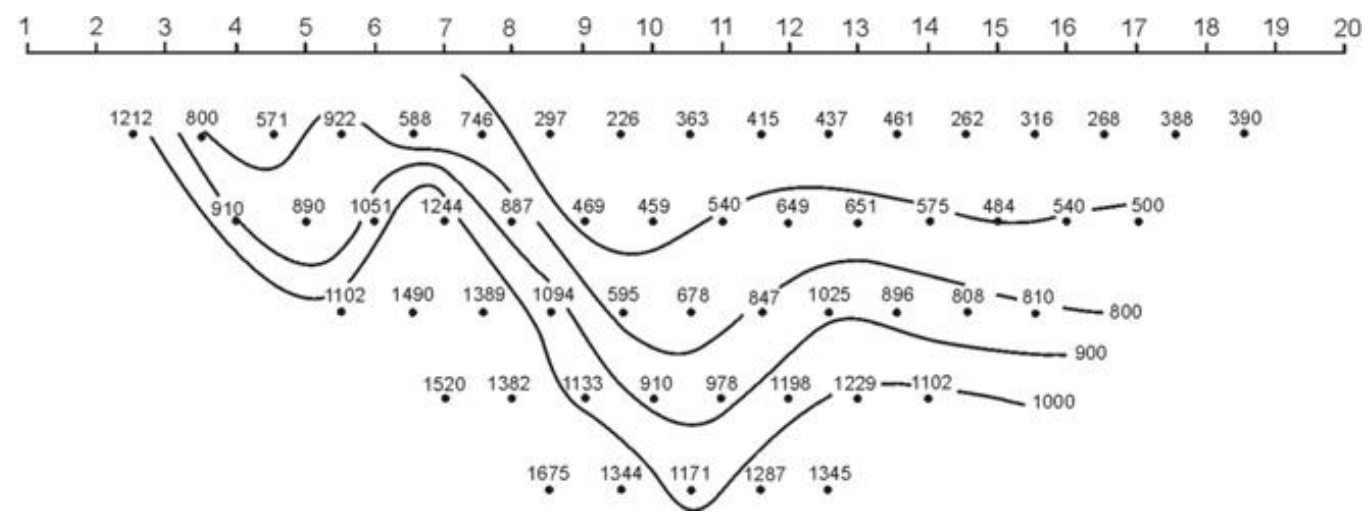

Figure 2.10: An example of a Wenner pseudosection(Barker, 2007).

\subsubsection{D and borehole techniques}

3D ERT images can be produced by either merging a number of parallel 2D ERT images or by making 3D measurements by arranging the electrodes in the form of a grid as shown in Figure 2.12 (Loke and Barker, 1996); (Loke, 1999). The first method is referred to as the quasi-3D technique (Dahlin and Loke, 1997). The measurements can be extended in a given direction by using the roll-along method as used in 2D imaging. 3D surveys are not very common because of the longer time involved in gathering data, the higher cost, and lack of necessary instrumentation and imaging software.

The spatial resolution of surface resistivity methods decreases with depth. A possible solution for this problem is to place the electrodes in boreholes. Placing electrodes in boreholes is convenient when it is not possible to place electrodes on the surface such as when there are buildings covering the surface. Common methods using boreholes are cross borehole ERT imaging, surface-to-hole configurations (Tsourlos et al., 2011) and mise-à-la-masse method(Rubin and Hubbard, 2005). Cross borehole measurements involve placing all the electrodes in the boreholes. Imaging using borehole electrodes is a straightforward extension of surface electrical imaging (Binley and Kemna, 2005). 

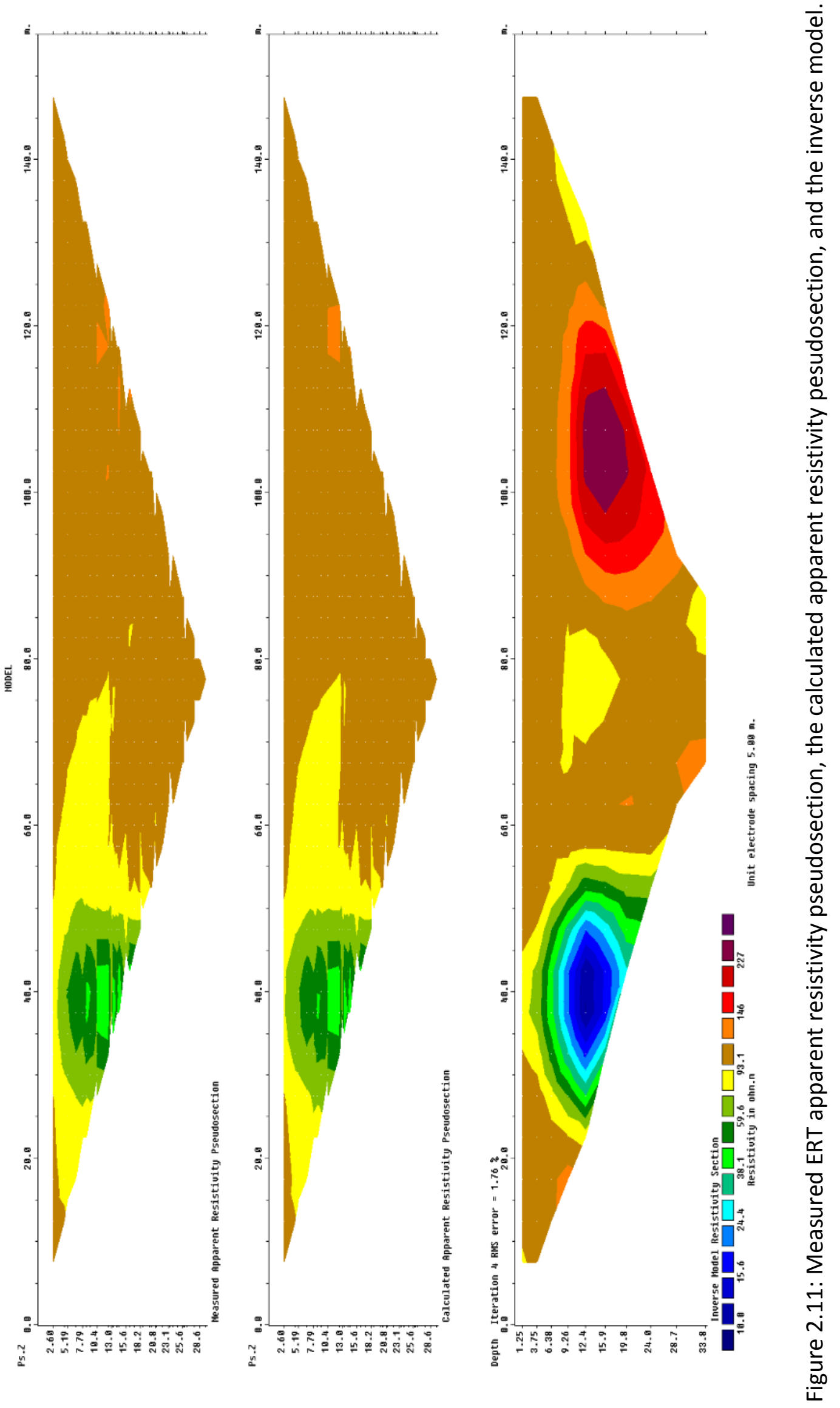
a)
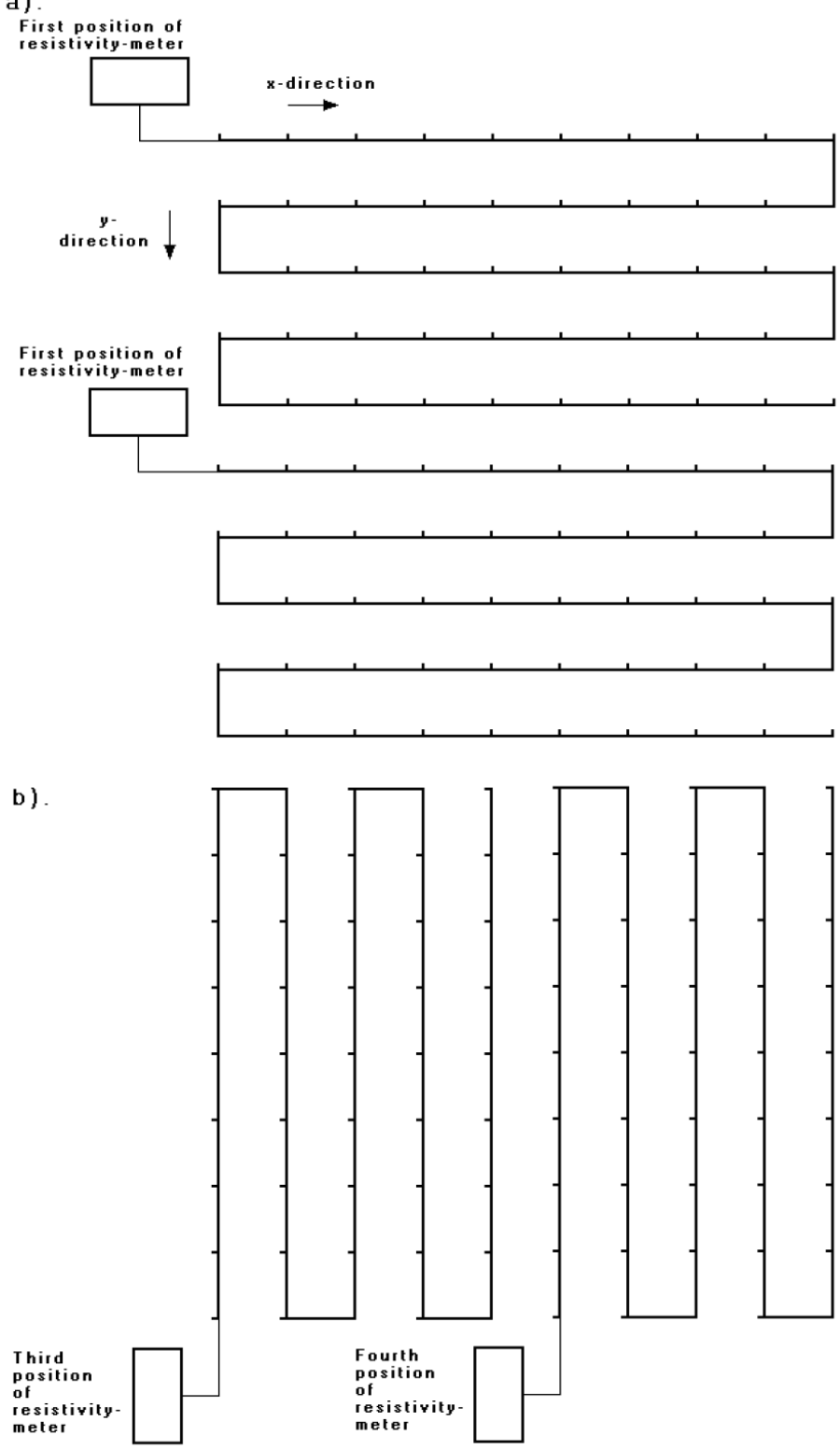

Figure 2.12: Using the roll-along method to survey a 10 by 10 grid with a 50 electrode system (Loke, 1999).

\subsubsection{Data interpretation}

In the early stages of using the DC resistivity method data interpretation was done by empirical methods, qualitative approaches, and the use of master curves. Some of the empirical methods adopted are the Moore cumulative resistivity method and the Barnes layer method (Burger et al., 2006). However, these methods are applicable only in the case of very simple resistivity distributions and they are not very reliable. In using a qualitative approach the shape of the sounding curve indicates the approximate number of layers present, and the relative values of the resistivity layers. The turning points in a 
sounding curve indicate the presence of subsurface layering. Therefore the number of layers is considered to be one more than the number of turning points in a sounding curve (Reynolds, 2011). An early quantitative approach to data interpretation was the use of master curves. The master curves are theoretically calculated sounding curves for a range of relative resistivity values which are expressed in terms of the reflection coefficients $k$. Figure 2.13 is a master curve for a two-layer model. As the number of layers increase master curves become cumbersome and hence are practical only up to four-layer models. In curve matching using master curves field data are plotted on a loglog graph on transparent paper. This paper is then placed on top of a set of master curves and moved to match a particular master curve and the respective $k$ value of the curve is noted.

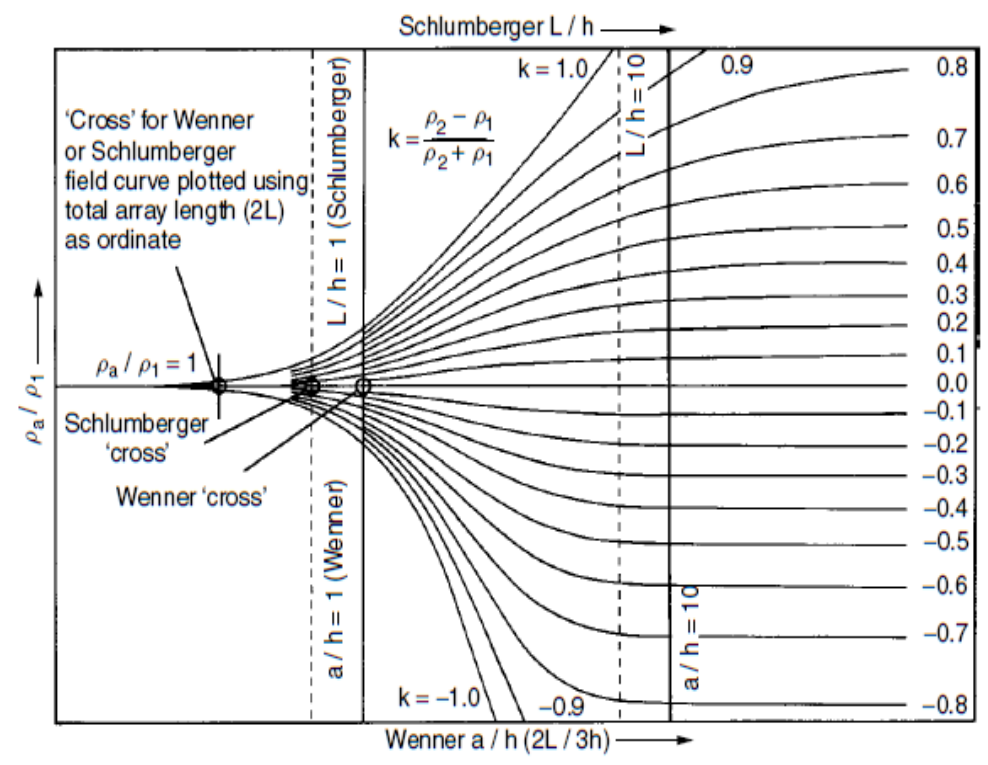

Figure 2.13: Two-layer apparent resistivity master curves for the Wenner array.

When matched to a field curve obtained over a two-layer earth, the line $a / h=1$ points to the depth of the interface and the line $\rho_{a} / \rho_{1}=1$ points to the resistivity of the upper layer. The value of $k$ giving the best fit to the field curve allows the value $\rho_{2}$ of the lower layer resistivity to be calculated (Reynold, 2011).

From 1970s computer programs have been used for curve matching. The computer program generates an apparent resistivity profile for a given set of resistivity values and layer thickneses. These computer generated models are then compared with field data and the difference between the modelled and field data is reduced by adjusting the layer thickness and resistivity values. There are various software packages that display some sort of statistical measure (for example the RMS error) that explains how well the 
modelled data correlate with the field data. The user can set this criteria and the program executes a number of iterations to match the criteria. For example in Figure 2.11 the RMS error is $1.76 \%$ and the number of iterations is 4 .

\subsubsection{Forward and inverse problem}

DC resistivity surveys aim to delineate the subsurface resistivity distribution from resistivity measurements. This process is not straightforward as the DC resistivity measurements give only the apparent resistivities. The theoretical outcome of an electrical resistivity survey can be mathematically determined or modelled by solving equation (2.1), subject to appropriate boundary conditions (Binley and Kemna, 2005). This process is called the "forward problem". Forward modelling involves estimation of apparent resistivities as measured by a DC resistivity survey when the resistivity distribution is known. The main methods used for forward modelling are (i) analytical methods (ii) boundary element methods and (iii) finite difference and finite element methods (Loke, 2004). Similarly from the data set from a DC resistivity survey the subsurface resistivity distribution can be directly derived; and this is called the "inverse problem" (Figure 2.14). Both forward and inverse modelling are difficult tasks because of non-uniqueness i.e. there exist a large number of models that will produce the same response. This is further complicated by limitations in data, and uncertainties involved in the measurements. Nowadays inversion is done by sophisticated software which use different approaches to solve the problem. The inversion software runs through a number of iterations of forward and inverse modelling until a satisfactory solution is obtained.

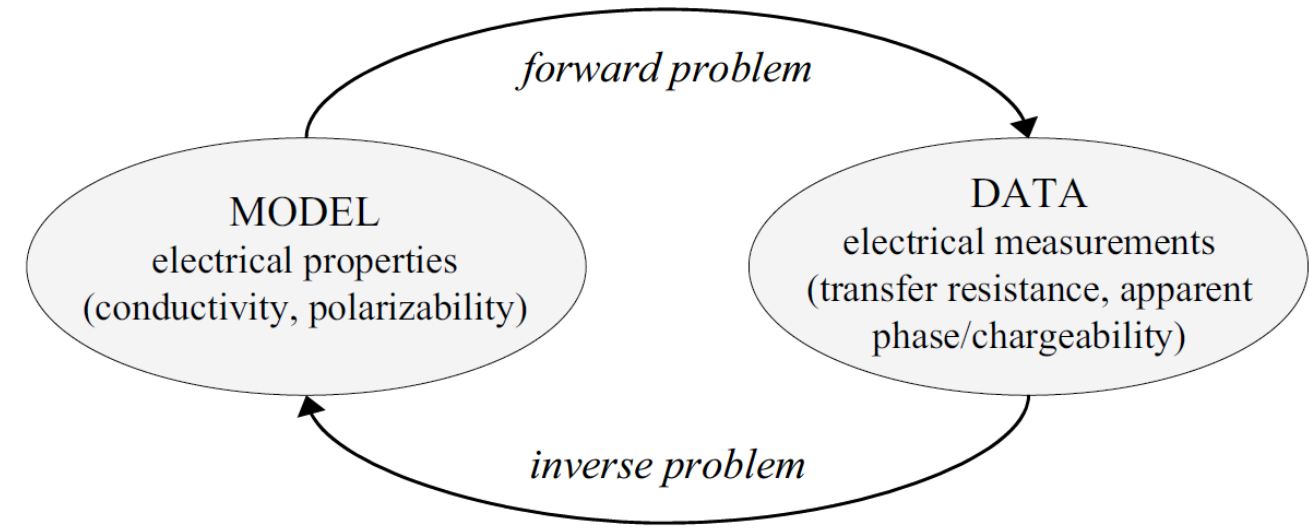

Figure 2.14: Definition of electrical forward and inverse problems. (Kemna et al. 2012) 


\subsection{Induced Polarization (IP)}

During DC resistivity measurements we have seen that the waveform of the voltage signal differs from that of the applied current signal as illustrated in Figure 2.5. The voltage between the potential electrodes does not become zero immediately when the exciting current source is switched off. Also, when the current source is switched on it takes a finite time to reach the maximum value of voltage. This behaviour is somewhat similar to that of a capacitor. The persistence of voltage between the potential electrodes, even after the current source is switched off, is because of the ability of the ground to polarize. This phenomenon is called the induced polarization (IP) effect. But the IP effect is not the same as the behaviour of an electrical circuit with a resistor and capacitor in parallel. In an RC circuit the voltage gradually decreases to zero from the maximum value. In the IP effect the voltage suddenly drops to a certain value and then decreases to zero as shown in Figure 2.15.

The credit for discovering the IP effect goes to Conrad Schlumberger who first observed the IP effect in 1912 (Sumner, 1976). IP is similar to the electrode polarization that occurs at the anode and cathode of an electrochemical cell when an electric current is passed through the cell (Bleil, 1953). The resistivity measured using the DC resistivity method is mainly controlled by ionic conduction through the pore fluids. This makes delineation of geological materials using resistivity alone a difficult task. It is possible that two different materials have the same resistivity because of variations in electrical conductivity of pore fluids. Usually such cases are resolved using DC resistivity in combination with other geophysical techniques. However, the sensitivity of the IP effect to the fluid-grain interface has the potential to improve the ability for delineation of geological materials. This has been used successfully to distinguish between different geological materials that have same resistivity (Slater and Lesmes, 2002). The low frequency capacitance of porous materials that causes the IP effect depends on their surface chemical properties. The slow decay of the voltage indicates the accumulation of charges at the grain fluid interfaces.

\subsubsection{Induced Polarization Measurement Systems}

Geophysical zinvestigations using the IP effect can be carried out by three different methods. These differ from one another in terms of the parameter used to measure the IP effect and to relate it to the geological properties, and in the instrumentation used. 
The commonly used measures of polarization are the chargeability (M), percentage frequency effect (PFE), and the phase angle $(\Phi)$. Chargeability is measured in the time domain while the other two are measured in the frequency domain.

(a)

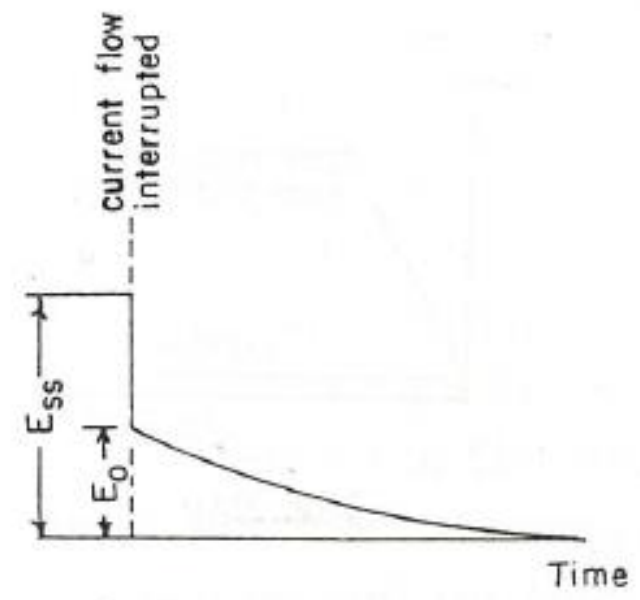

(b)

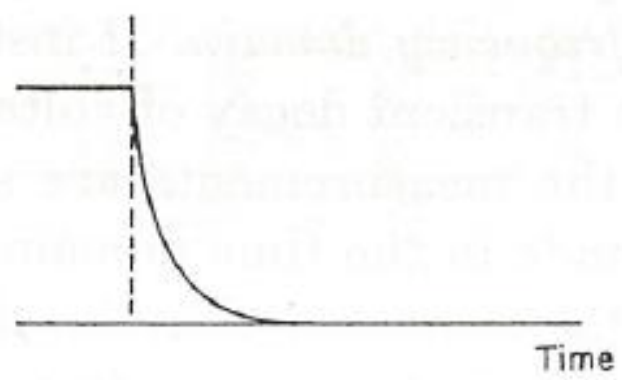

Figure 2.15: (a) Transient decay of electric field strength in a rock sample. (b) Transient decay of voltage in an RC circuit. (Keller and Frischknecht, 1966).

\subsubsection{IP in the time-domain}

In time-domain IP a steady current is applied as in the case of DC resistivity. When the current is switched off there will be a slow decay of voltage at the potential electrode as shown in Figure 2.15. The voltage measured while the current is flowing is referred to as the primary voltage $\left(\mathrm{V}_{p}\right)$. As soon as the current is switched off, the voltage suddenly drops to a value referred to as the secondary voltage $\left(V_{s}\right)$ and then slowly decays to zero as in the case of a discharging capacitor. The ratio of the secondary voltage to the primary voltage is a measure of the chargeability, called the apparent chargeability $M_{a}$ ， given by Siegel (1959) as

$M_{a}=\frac{V_{s}}{V_{p}}$ 
As it is difficult to measure the secondary voltage accurately in the field (Binley and Kemna, 2005), chargeability is often calculated using the relationship

$M_{a}=\frac{1}{\left(t_{2}-t_{1}\right)} \frac{1}{V_{p}} \int_{t_{1}}^{t_{2}} V(t) d t$

where $t_{1}$ and $t_{2}$ are as shown in Figure 5 . Here the chargeability is given by the integrated amplitude of the decay curve after the excitation current is switched off, normalized with respect to the excitation voltage. The unit of chargeability is $\mathrm{mV}^{-1} \mathrm{~V}^{-1}$. In modern instruments the voltage decay is recorded using a very high sampling rate and hence the frequency domain properties can also be determined using Fourier transformation. Chargeability is a useful parameter for distinguishing between different kinds of materials.

\subsubsection{IP in the frequency-domain}

In the frequency-domain, subsurface apparent resistivity is measured at different frequencies using the same electrode array as in the case of time domain IP and DC resistivity measurements. Induced polarization causes a variation of apparent resistivity as the frequency of the excitation current varies. The apparent resistivity measured at high frequencies is smaller compared to that at lower frequencies. A measure of the IP effect in the frequency-domain is the percentage frequency effect (PFE) given by

$P F E=\frac{\rho_{a 0}-\rho_{a 1}}{\rho_{a 1}} X 100$

where $\rho_{a 0}$ and $\rho_{a 1}$ are apparent resistivities at low and higher frequencies respectively. It is found that a frequency of $0.05 \mathrm{~Hz}$ for lower frequency and $10 \mathrm{~Hz}$ for higher frequency is a good choice to measure the frequency effect. But in practice frequencies higher than $0.05 \mathrm{~Hz}$ and lower than $10 \mathrm{~Hz}$ are used for lower and higher frequencies respectively because of time constraints of the survey (Beck, 1981).

\subsubsection{Spectral Induced Polarization (SIP)}

IP surveys that provide the apparent chargeability $\left(M_{a}\right)$ and percentage frequency effect (PFE) are mainly used for identifying metallic minerals. More detailed information about the nature of the porous medium that has potential to provide important hydrological information can be obtained by measuring the complex conductivity of the porous medium (or, alternatively, its impedance or admittance). Here the term complex is used to indicate that the measured quantity has both a real and imaginary part. This technique, known generally as spectral induced polarization (SIP), involves measurement of complex electrical conductivity at a very large number of frequencies 
spanning from about $1 \mathrm{mHz}$ to $1 \mathrm{kHz}$. Often the selected frequencies are ten logarithmically equally spaced frequencies per decade.

In complex conductivity measurements, a sinusoidal current is injected compared to the square waves used in time-domain and frequency-domain IP. The capacitive nature of the porous medium introduces a phase difference between the injected current and the measured voltage waveform between the potential electrodes as shown in Figure 2.16. The instrumentation used in SIP measurements is much more sophisticated than for other electrical methods. In SIP measurements the instrument stores both the injected current and measured voltage waveforms in digital form. By analysing the digitised waveforms the impedance is calculated in terms of its modulus and phase. The real and imaginary part of the complex conductivity are then calculated from the knowledge of the geometric factor; which depends on the type of electrode array and spacing between the electrodes.

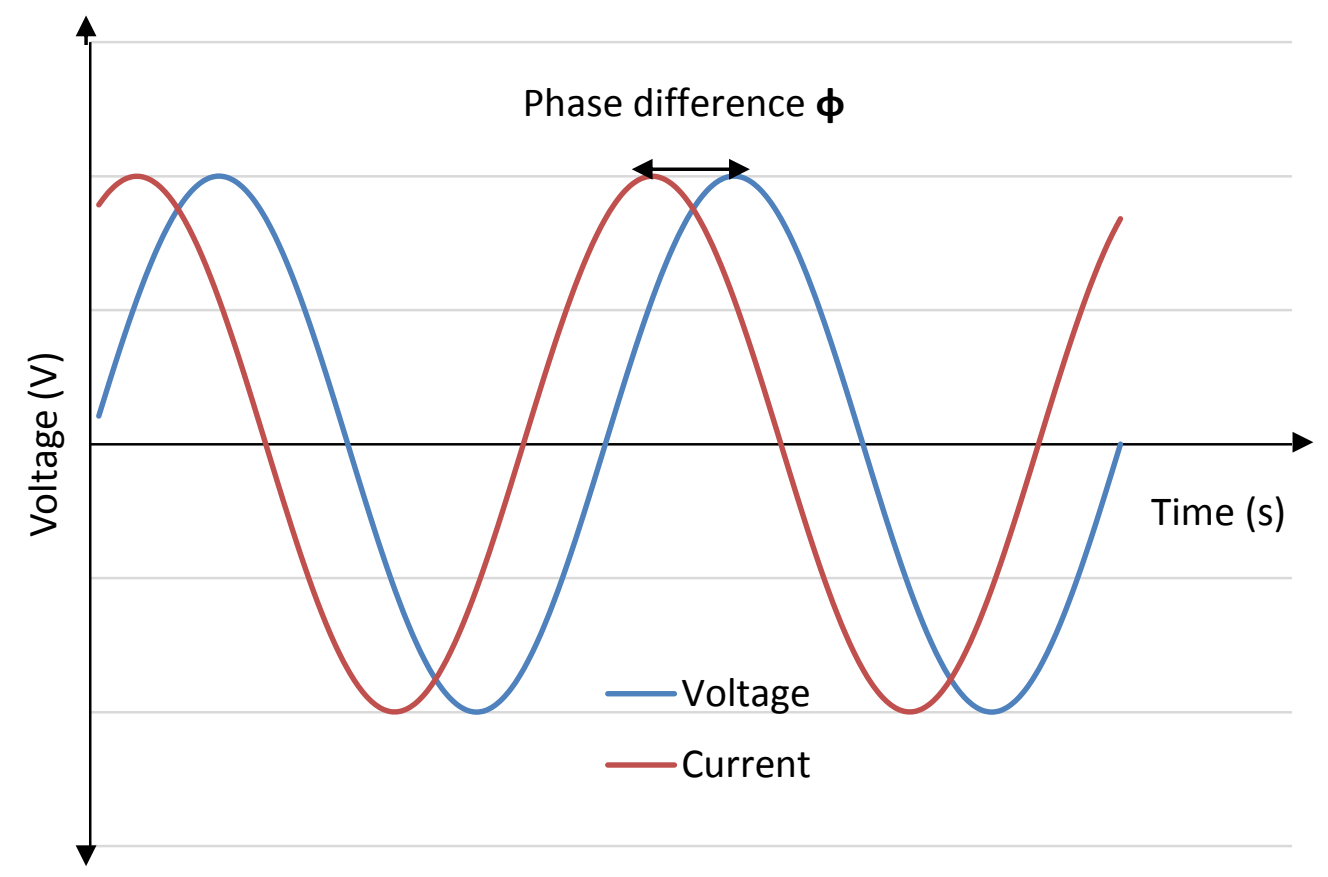

Figure 2.16: Current and voltage waveforms in SIP measurements showing a phase difference.

\subsection{History of Spectral Induced Polarization}

The very first observation of the IP effect is attributed to Conrad Schlumberger. He noticed it as early as 1912 and correctly identified it as an electrochemical effect. After 
some initial trials Schlumberger gave up attempts to use IP for mineral prospecting as he noticed that both mineralized and unmineralized rocks exhibited the IP effect. Thereafter, the IP phenomenon, similar to that observed by Schlumberger, was noted by other workers who were possibly unaware of his original work. Hence the development of IP for geophysical prospecting has a number of relatively independent roots. These developments took place mainly in the USSR, in the US Naval Ordnance Laboratory, in mining companies, and in universities in the United States. Siegel et al. (2007) give a detailed description of the early development of the induced polarization method.

IP related research in USSR started with the observations of D.M. Murashov in 1927. Scientists who followed up the work of Murashov investigated the dependence of the IP effect on the density of polarizing current, the time of establishment, the composition of the sulphides, and the nature of electrolyte in contact with the minerals. IP effects of non-sulphide rocks and sandy clays were also studied. V. A. Komarov and Y. S. Ryss were able to develop a method to distinguish between mineralized and unmineralized rocks from their IP response; the problem that earlier forced Schlumberger to abandon IP. With continued support from theoretical and experimental research, by the mid-1960s IP became one of the prominent tools for exploration of sulphide related minerals in USSR.

It was the 1942 US navy project to develop a mine locator using IP that created renewed interests in IP in North America. The scientists who worked on the mine locator project continued their research in IP after they left the US Naval Ordnance Laboratory. This led to spreading and adaptation of IP techniques for geophysical applications. Though the mine detector project was top secret one of the scientists involved, David Bleil was permitted to complete a PhD in the subject area. Bleil investigated the possibility of using IP for locating buried minerals. His work clearly established the capability of IP as a tool for geophysical prospecting. The summary of his PhD thesis named "Induced Polarization, a method of geophysical prospecting" (Bleil, 1953), which was published in the journal Geophysics, inspired many to further develop IP method; and is considered as the first publication about IP in North America. It was Bleil who coined the term "Induced Polarization". 
In an attempt to develop new techniques for mining, the New York based Newmont Mining Corporation came across the IP phenomenon. They developed necessary instrumentation for applying IP for field work. The PhD work of Harold Siegel, in association with Newmont Mining Corporation, was an important milestone in the application of IP for prospecting. His work identified the contributions to IP from nonmineralized sources also. Newmont Mining Corporation established a team of scientists and engineers who successfully developed IP for commercial applications. The research work at Newmont is summarised in the book "Overvoltage Research and Geophysical Applications" edited by James R. Wait (Wait, 1959). The Newmont team were the first to make an attempt at Spectral Induced Polarization even though they did not pursue it further.

Like Newmont, another mining company Anaconda Copper Company was also interested in using IP for prospecting. Under Ed McAlister, who was also part of the team that developed the mine locator for US Naval Ordnance Laboratory, Anaconda developed necessary instruments and field techniques in IP. They were the first to use a telluric cancelling system to improve the signal/noise ratio.

Inspired by the work of David Bleil, and with support from the U.S. Atomic Energy Commission, Ted Madden of Massachusetts Institute of Technology formed a team of students that conducted research in IP. Madden pioneered the use of sine wave current forms. It was Ted Madden's team that first identified other potential mechanisms of IP like membrane polarization. They introduced the terms "percent frequency effect" and "metal factor".

The main aim of the IP works discussed above was mineral discrimination. IP was most successful in the prospecting of porphyry copper deposits which were not responsive to other geophysical techniques like electrical, electromagnetic, gravity or magnetics (Siegel et al., 2007). Even though the possibility of frequency domain measurement was known, it did not get much attention mainly because of the complexities in instrumentation. The potential for the application of IP to detect groundwater was investigated by a group at New Mexico Institute of Mining and Technology under Victor Vacquier (Vacquier et al., 1957). It was the work of Kenneth Zonge and W. H. Pelton that pioneered the development of Spectral IP. Zonge developed instruments for complex resistivity measurements, and found a practical general solution to the problem of EM 
coupling. He went on to establish the Zonge Engineering and Research Organization. The seminal paper "Mineral Discrimination and Removal of Inductive Coupling with Multifrequency IP" (Pelton et al., 1978) by Pelton, and his use of the Cole-Cole model made Spectral Induced Polarization measurements popular for mineral discrimination. Research and development in the application of IP continued till the late 1970s. But the crash of copper prices in 1983, followed by decline in oil prices in 1985 (Mathews and Zonge, 2003), caused a decline in IP research activities. Irrespective of the loss of commercial importance, there was a steady increase in the capability of SIP instrumentation; fuelled by the advancements in microprocessor and computing technology.

SIP is now used increasingly for hydrogeophysical, environmental and biogeophysical applications. In the early years, however there was hardly any attempt for using SIP for groundwater studies other than the work of Vacquier et al. (1957). More recently Frank D. Börner (Börner, 1992) used IP for a number geophysical investigations starting in the early 1990s.

\subsection{Conclusion}

This chapter introduces four electrical methods widely used in geophysics, the selfpotential method, the DC resistivity method, the Induced Polarisation method and the Spectral Induced Polarisation method. The DC resistivity method has a lot in common with the SIP method. The electrode configurations, survey configurations, tomographic techniques, and borehole techniques are all also applicable in principle for SIP surveys. An SIP survey provides all the details that a DC resistivity survey provides and some additional information about the surface properties of the medium. The difference between DC resistivity and an SIP survey is that in an SIP survey the measurements between two electrodes are repeated at a number of frequencies compared to a single measurement in the case of DC resistivity. The DC resistivity instruments use a low frequency square wave to record the amplitude of the signal while an SIP instrument uses a sine wave signal and records both the amplitude and phase difference or the quadrature component of resistivity. It is this quadrature component of resistivity that is ,believed to be controlled by the surface properties of the porous medium, that provides additional information about the medium which can act as a proxy for hydraulic 
properties like hydraulic conductivity. This chapter end with a note on a short history of the development of SIP. 


\section{SIP Mechanisms and SIP spectra models}

\subsection{Introduction}

The Induced Polarization phenomenon is understood in terms of the transport and distribution of charge carriers around the mineral surfaces in porous media under the influence of an external electric field. Even though the phenomenon of IP has been around for about 100 years, there are no clear models that explain the origins of spectral induced polarization. One thing that makes the interpretation of observed phenomenon of IP challenging is that there are many competing mechanisms. These include electrode polarization, membrane polarization, electrical double layer polarization and MaxwellWagner polarization that might be acting either alone or in parallel. It is difficult to segregate the effects of each of these different mechanisms.

Various international workshops have reiterated the necessity to develop a mechanistic model for describing Induced Polarisation. Ward et al., (1995) summarise the recommendations of the John S. Sumner Memorial International Workshop on Induced Polarization in Mining and the Environment held 17-19 October 1994 in Tucson, Arizona. They say that "more theoretically-based physiochemical models are needed to relate directly the measured complex electrical properties of rocks to their specific microgeometrical and surface chemical properties. Progress in this direction may also lead to better understanding of the Cole-Cole empirical parameters in terms of the physiochemical properties of rocks". Although a lot of work has been done on this since 1995 , there is no comprehensive mechanistic model that describes the IP mechanism.

\subsection{Induced Polarization mechanisms}

The frequency dependent variation of impedance in SIP cannot be explained in terms of the atomic or molecular structure of the material, but appears to depend on the texture or microstructure of the porous medium. The microstructure of a porous medium is defined by the grain size, grain shape, pore size, and the pore throats as illustrated in Figures 3.1 and 3.2. Such a microstructure where there may be grains of different types of minerals responds to the flow of charges through the medium resulting in charge accumulation and separation at the interfaces. This polarization of charges influences the impedance of the medium. Hence the exact mechanism that gives rise to SIP may depend on the size and nature, size and shape of the grains, the pore size and the pore 
throats.

Kemna et al. (2012) have identified the following five main mechanisms that contribute to the SIP response;

(1) Electrode polarization,

(2) membrane polarization; the

(3) Maxwell-Wagner Polarization;

(4) polarization of the diffuse layer, i.e., the outer part of the electrical double layer (EDL); and

(5) polarization of the Stern layer, i.e., the inner part of the EDL at the interface between minerals and water. These will be defined and elaborated in the next section.
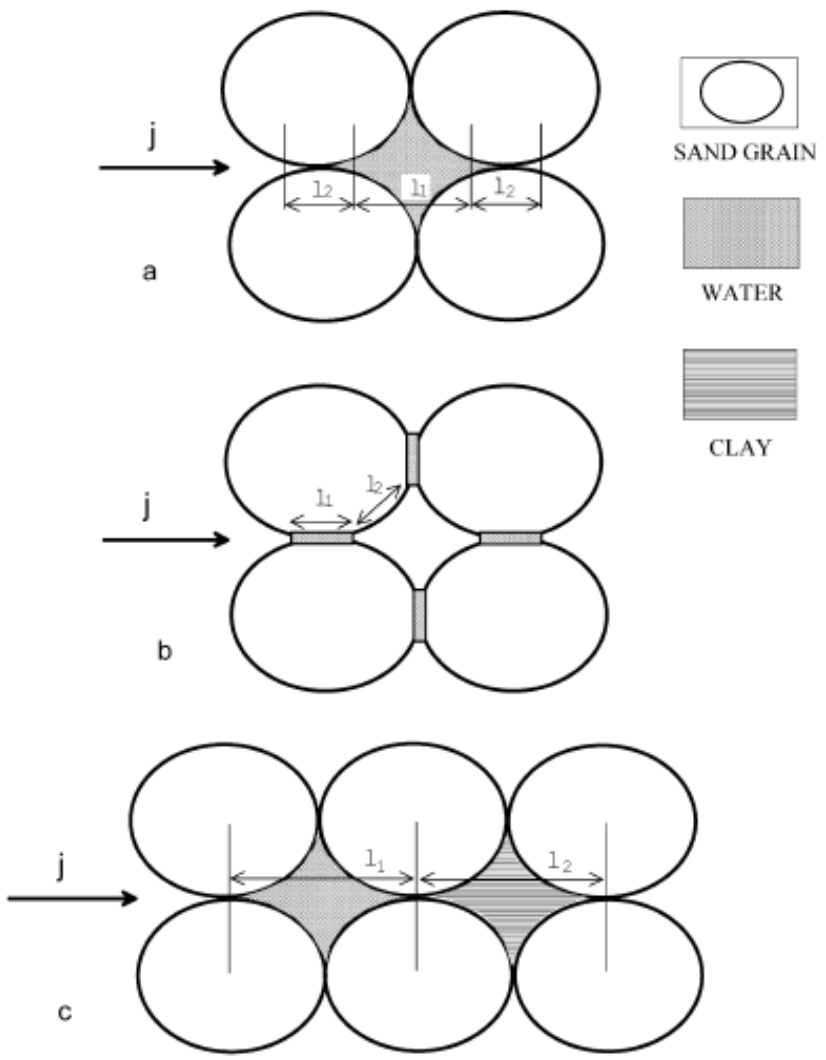

Figure 3.1: Schematic of the geometry of current passages in sandy-clay sediments. (a) Pure water-saturated sand, (b) pure unsaturated sand, (c) saturated sandy-clay sediments. In the saturated sand, water filled spaces can be considered as large pores of length $l_{1}$. Areas of grain contact where the water is in a closer bond on the grain surface, can be considered as narrow pores of length $I_{2}$. In the unsaturated sand the water film at the surface of moistened grains can be considered as narrow capillaries of length $I_{2}$, while areas of grain contact can be considered as large capillaries of length $I_{1}$. In the sandy-clay saturated sediments the large passages are water filled pores between the grains, while the narrow pores are filled with clay particles (Titov et al. 2002). 


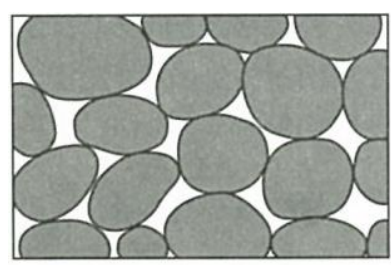

(a)

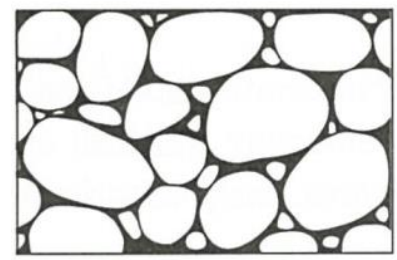

(d)

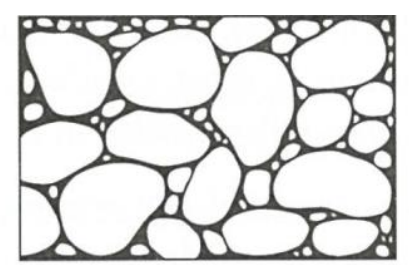

(b)

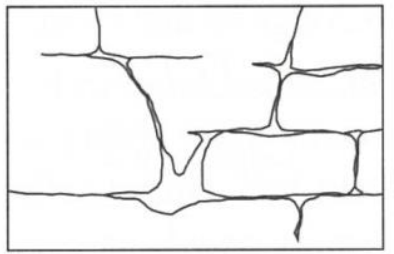

(e)

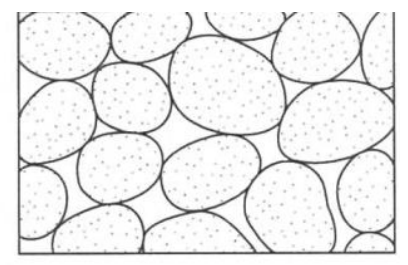

(c)

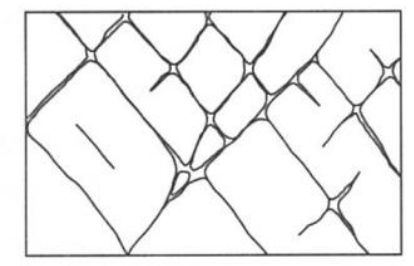

(f)

Figure 3.2: Examples of rock interstices and the relation of rock texture to porosity.

(a) Well-sorted sedimentary deposit having high porosity. (b) Poorly sorted sedimentary deposit having low porosity. (c) Well sorted sedimentary deposit consisting of pebbles that are themselves porous, so that the deposit as a whole has very high porosity. (d) Well sorted sedimentary deposit whose porosity has been diminished by the deposition of mineral matter in the interstices (e) Rock

\subsubsection{Electrode polarization or overvoltage}

When a metal electrode is immersed in an electrolyte, separation of charges surrounding the metal takes place as shown in Figure 3.3. This separation of charges leads to the establishment of a potential difference between the metal and the electrolyte. The charge distribution has the form of an electrical double layer. The electrical double layer consists of an adsorbed layer and a diffuse layer of ions. The net potential difference between the electrolyte and the solution is called the Nernst potential, and the contribution from the adsorbed layer is called the zeta potential. Application of an external voltage can disturb the existing charge distribution and cause a current flow. When the external voltage is removed, the charge distribution returns to the initial state by the diffusion of ions.

The presence of metallic mineral grains in a geological material can create centres of electrode polarization as explained above. The most common example of this is when disseminated metallic sulphides are present in rocks. The majority of the electrical conduction in such a rock is ionic in nature, and takes place by the movement of ions in the electrolyte that fills the pores in the rock. But it is possible that such flow paths of 
ions can be blocked by the presence of metallic grain as shown in Figure 3.4. This causes accumulation of charges, which in turn increases the potential difference between the metallic particle and the electrolyte. As the 'pile-up' of charges leads to an increased potential difference between the grain and electrolyte, the phenomenon of electrode polarization is also called the overvoltage (Beck, 1981). Upon the removal of an applied external voltage, the ions diffuse back to initial state and the overvoltage disappears. This slow diffusion of ions give rise to the characteristic overvoltage decay recorded in a time-domain IP measurement. The factors affecting the rate at which the ionic balance is restored are extremely complex and may depend upon the pore shape and size, rock structure, permeability, electrolytic conductivity, ionic concentration and the electronic conductivity of the mineral grain (Reynolds, 2011).

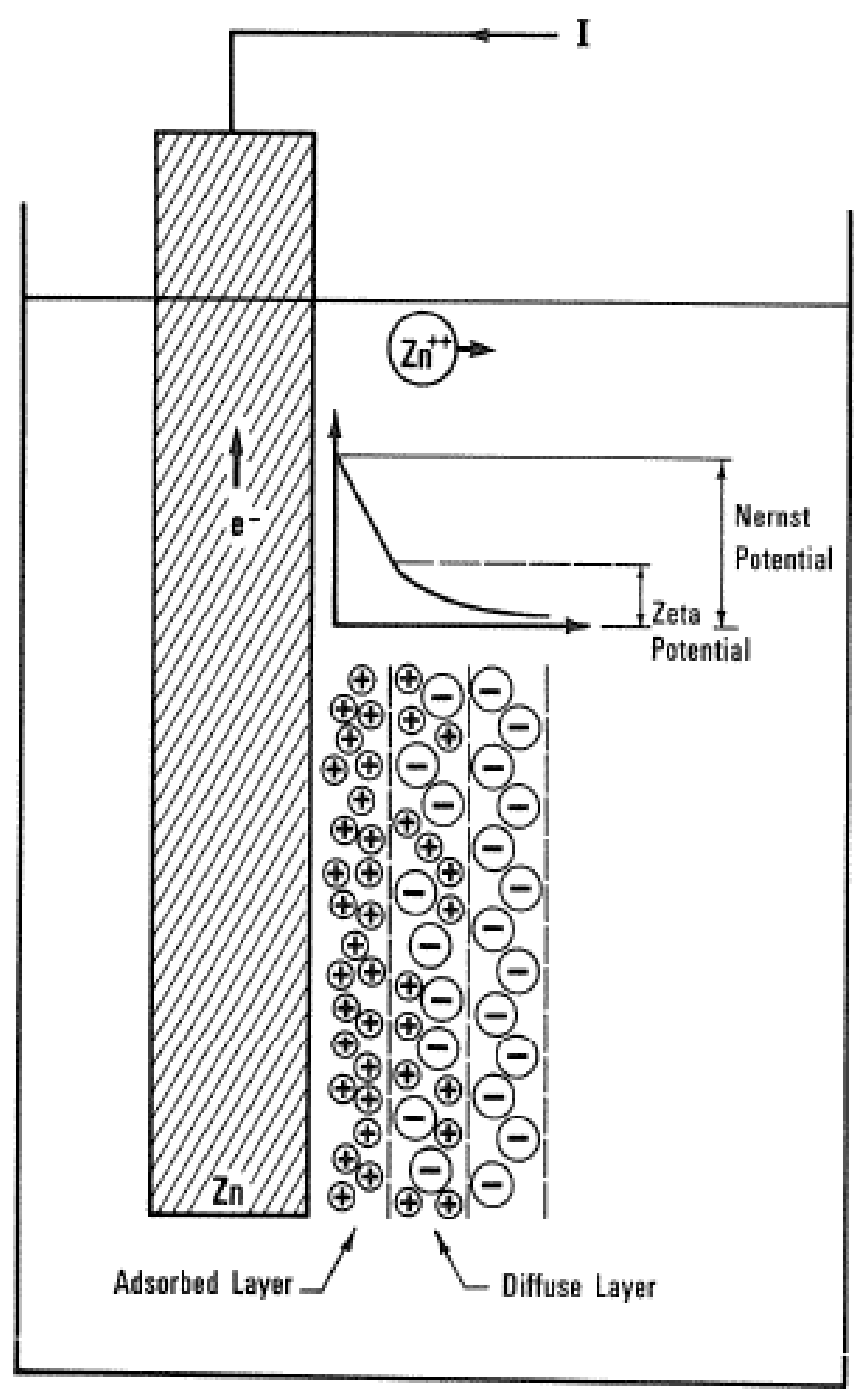

Figure 3.3: Illustration of elements of electrode polarization (Beck, 1981). 


\subsubsection{Membrane or electrolytic polarization}

The second type of polarization mechanism is membrane polarization. There are two possible causes for membrane polarization; the constrictions in pore channels, and the presence of clay in pore channels. Mineral surfaces have a tendency to develop a negative charge. This causes the accumulation of positive charges on mineral surfaces as shown in Figure 3.5(A). This concentration of positive ions can extend up to $100 \mu \mathrm{m}$

(A)

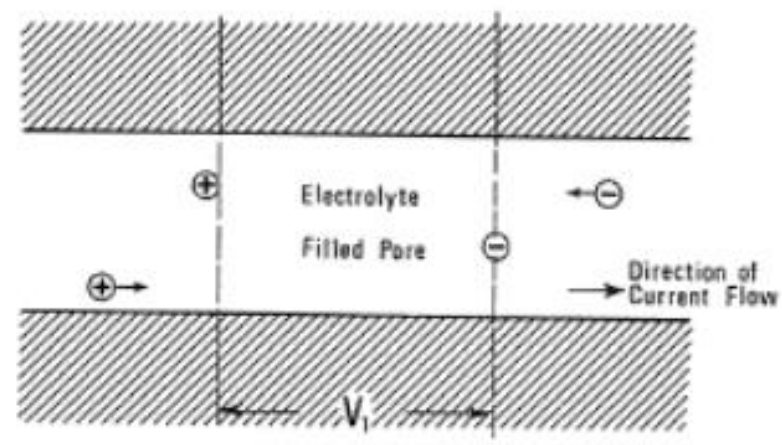

(B)

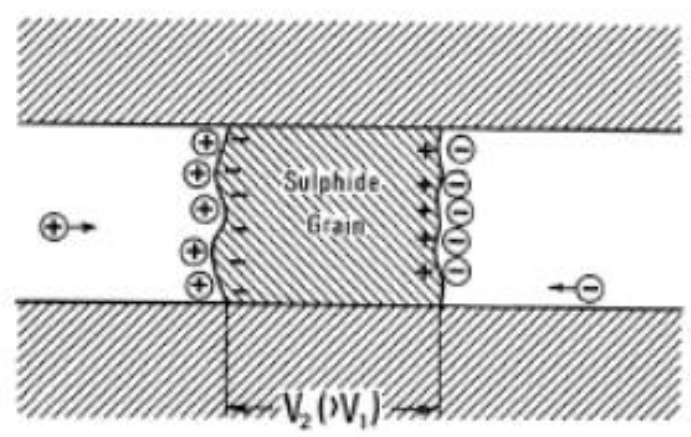

(C)

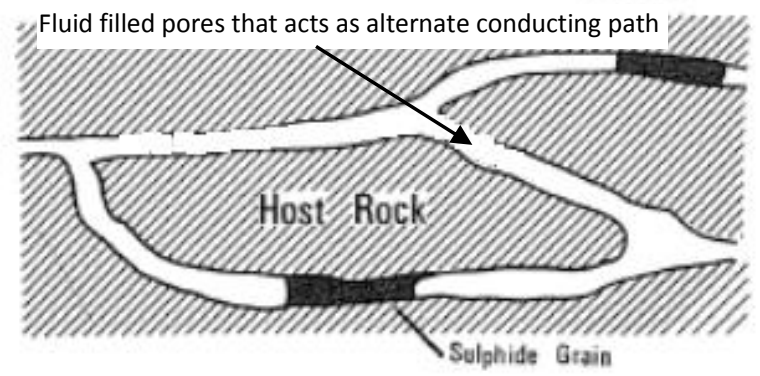

Figure 3.4: (A) Fluid filled pore in a rock indicating potential, $V_{1}$, which could be measured between two planes in conditions of free ionic motion. (B) Same pore as in $(A)$ but blocked by a sulphide particle between the planes in (A). Ionic charges build up, a new potential, $V_{2}\left(>V_{1}\right)$, would be measured, and "overvoltage" develops. (C) General scheme for alternate ionic paths when some pores are blocked by sulphide particles (Beck, 1981). 
into the pore fluid from the mineral surface. If the sizes of pore throats are the same size as the positive charge layer this can prevent the movement of ions when a voltage is applied. As a result, negative and positive ions build up on either side of the pore producing a potential difference across the blockage as shown in Figure 3.5(A). Once the external voltage is switched off the charge distribution returns to its initial state by diffusion which produces the measured IP response (Reynolds, 2011).

The presence of clay particles or filaments of fibrous minerals in the pore channel can cause an accumulation of negative charges (Figure 3.5(B)). These negative charges in turn attract positively charged ions creating a positively charged cloud surrounding the negatively charged particles. When an external voltage is applied the positive charges can move between the similarly charged clouds but the negatively charged ions are blocked and this produces a difference in ionic concentration. When the external voltage is switched off, the ions diffuse back to attain the initial state which give rise to a measurable IP response.

\subsubsection{Maxwell-Wagner Polarization}

The third type of polarization mechanism is called Maxwell-Wagner Polarization. Maxwell-Wagner Polarization is an interfacial polarization caused by the discontinuity of displacement currents in a multiphase system with discontinuities of dielectric permittivity and/or electrical conductivity at the interphase between the different phases. This mechanism is controlled by the tortuosity of the different phases, their volume fractions and the conductivity and permittivity of the different phases. MaxwellWagner Polarization is mainly responsible for polarization phenomena at the upper end of the considered frequency spectrum (typically above $1 \mathrm{kHz}$ (Kemna et al., 2012).

\subsubsection{Polarization of diffuse layer}

In unconsolidated sediments the polarization is mainly associated with the electrical double layer surrounding the mineral grains. Hence the mechanism of polarization is slightly different. Figure 3.6 shows the formation of an electrical double layer around a mineral grain. When a mineral grain is immersed in water, the chemical reaction between water and certain functional groups like silanol and aluminol (Revil and Florsch, 2010) leads to the formation of a net surface charge on the grain. This net surface charge 


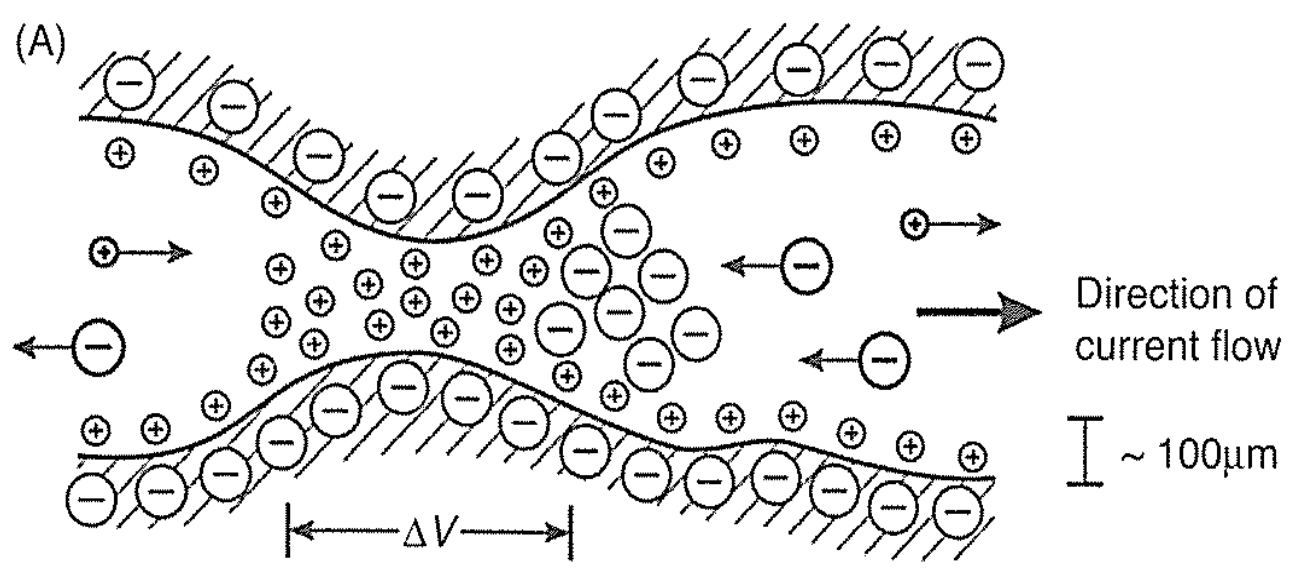

(B)

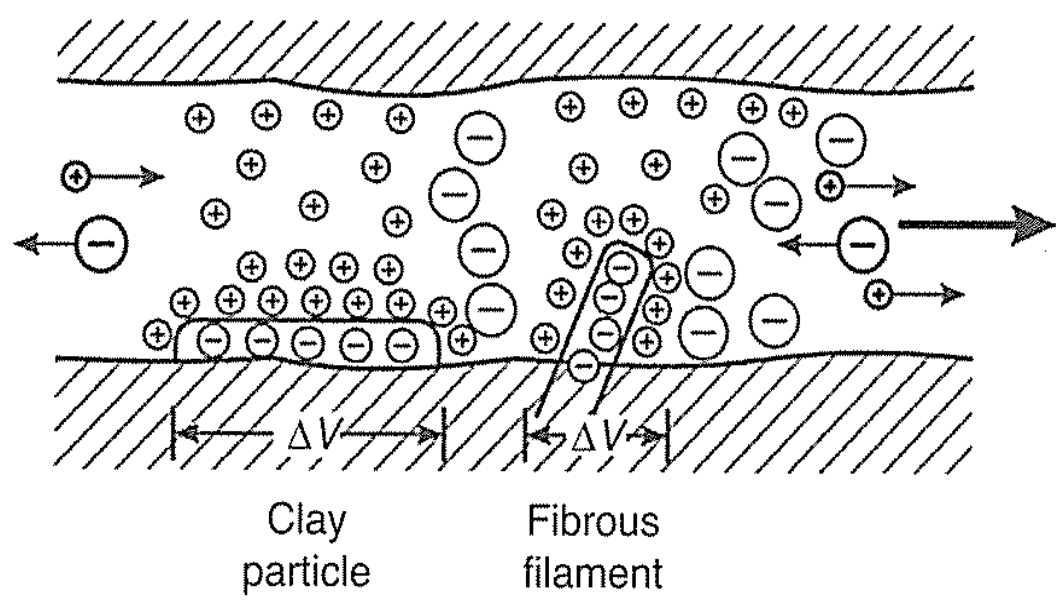

Figure 3.5: Development of membrane polarization associated with (A) a construction within a channel between mineral grains, and (B) negatively charged clay particles and fibrous elements on the sides of a channel (Reynolds, 2011).

on the grain causes the formation of a layer of counter ions (counter ions are ions that have the opposite charge to the mineral surface) that surrounds the mineral grain. This layer is called the Stern layer. But the Stern layer alone does not balance the net surface charge on the grain. This leads to the formation of another layer surrounding the Stern layer, called the diffuse layer. Unlike the Stern layer, coions (ions that have the same surface charge as the mineral surface) are also present in the diffuse layer though in a minority. This distribution of charges surrounding a grain is called the electrical double layer. According to Revil and Florsch, (2010) the condition for polarization of the diffuse layer is that the grains are far enough apart that the diffuse layer does not form a 
continuum. Thus when the grain density is small the polarization of the diffuse layer can take place. Polarization of the electrical diffuse layer can cause an increase of the salinity on one side of the grain and a decrease of the salinity on the other side of the grain as shown in Figure 3.7. (See Revil and Florsch (2010) for the detailed explanation of diffuse layer polarization). This difference in salinity causes a back diffusion through the pore space and this can give rise to an IP response. This sort of polarization of the diffuse layer can lead to membrane polarization as discussed in section 3.2.2 (Revil and Florsch, 2010).

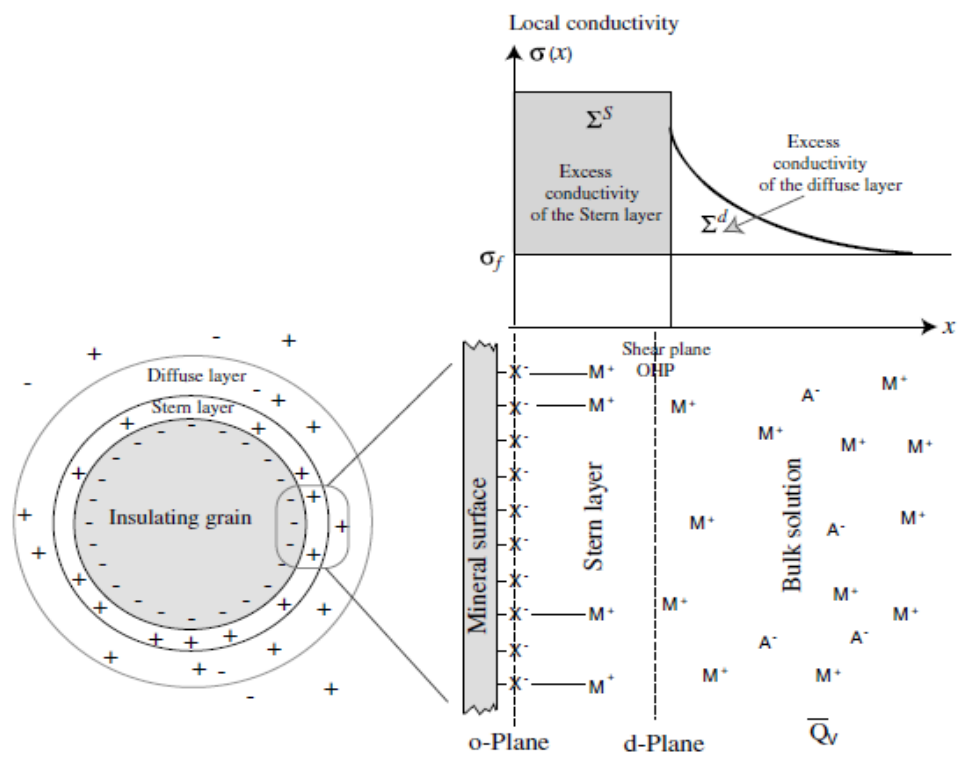

Figure 3.6: Sketch of ionic species in the pore space of a charged porous medium at equilibrium. The pore water is characterised by a volumetric charge density $\bar{Q}_{v}$ corresponding to the charge of the diffuse layer per unit pore volume (in $\mathrm{Cm}^{-3}$ ). The Stern layer is responsible for the excess surface conductivity $\Sigma^{S}$ (in S) with respect to the conductivity of the pore water $\sigma_{f}$ while the diffuse layer is responsible for the excess surface conductivity $\Sigma^{d}$. The Stern layer lies between the o-plane (mineral surface) and the d-plane, which is the inner surface of the electrical diffuse layer. The diffuse layer extends from the $d$-plane to the pores (Revil and Florsch, 2010).

\subsubsection{Polarization of the Stern layer}

The counterions in the Stern layer are held firmly to the mineral surface by the electrostatic force of attraction; but they are free to move in a direction tangential to the surface of the mineral grain. So when an external electric field is applied, the Stern 


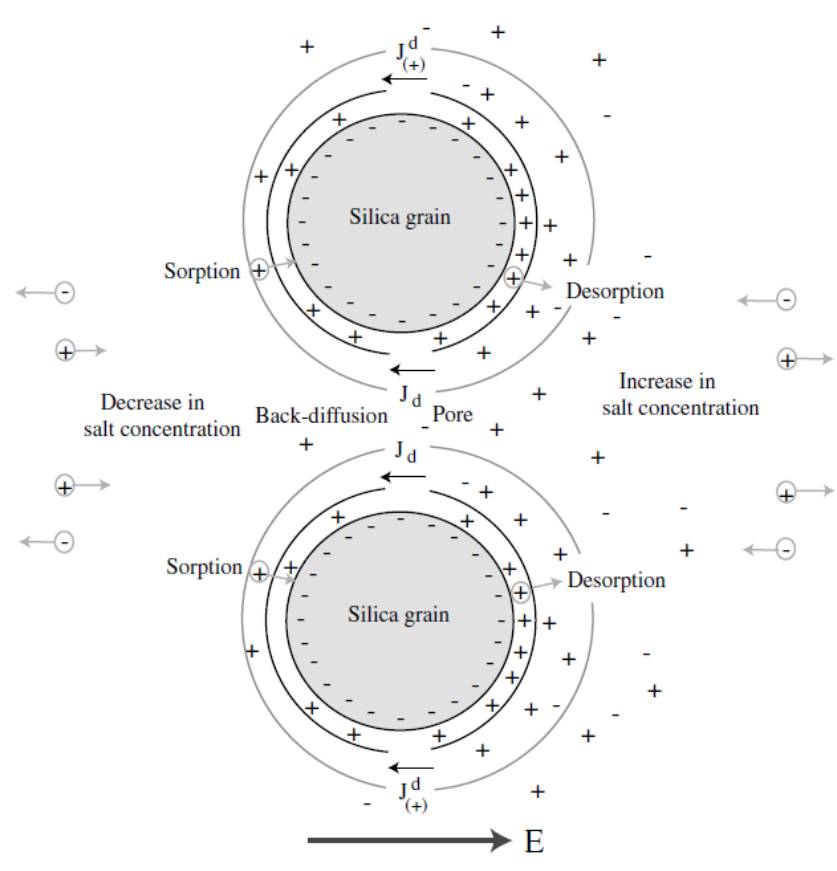

Figure 3.7: Sketch of membrane polarization of a pore sandwiched between two grains. The back-diffusion can occur both in the electrical double layer for the counterions and in the bulk pore water for the coions. The salt accumulated on one side of the pore diffuses back with a diffusion coefficient that is equal to the mutual diffusion coefficient of the salt (Revil and Florsch, 2010).

layer becomes polarized. However, there is a possibility of back diffusion in the Sternlayer also (Revil and Florsch, 2010). This back-diffusion of counter ions in the Stern layer can also generate an IP response.

\subsection{The influence of the nature of the medium on the SIP mechanism}

The predominant mechanism of induced polarization also depends to some extent on the nature of the porous medium. Figure 3.8 describes the three different scenarios where the leading IP mechanism is different because of the pore structure of the medium under consideration. As shown in Figure 3.8, polarization of the diffuse layer is an important contributing mechanism in colloidal suspensions; while it is the Stern layer polarization that is predominant in a dense collection of mineral grains. When it comes to a consolidated porous medium like rock diffuse and layer Stern layer polarization are not that relevant, and membrane polarization dominates the SIP response. 
Type A. Colloidal suspension

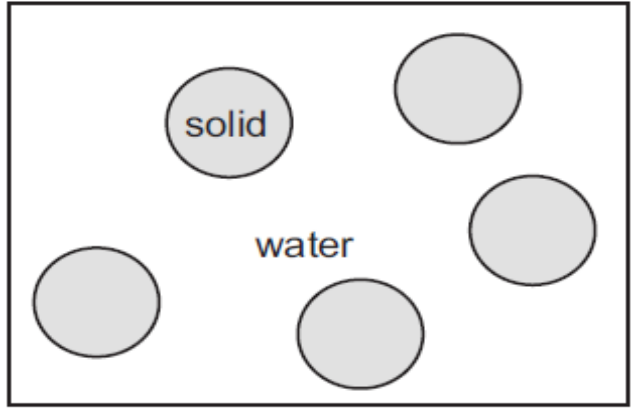

Type B. Granular porous medium

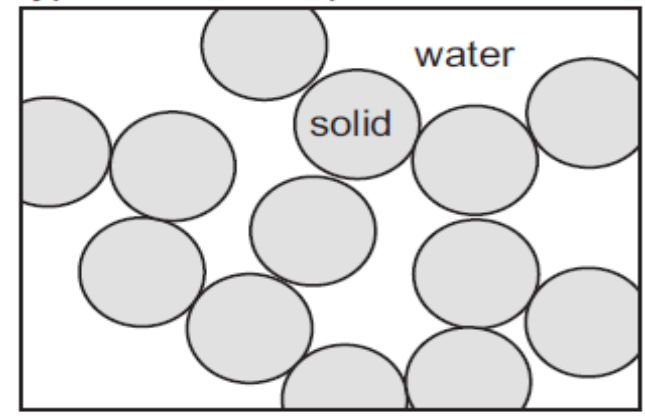

Type C. Continuous solid phase

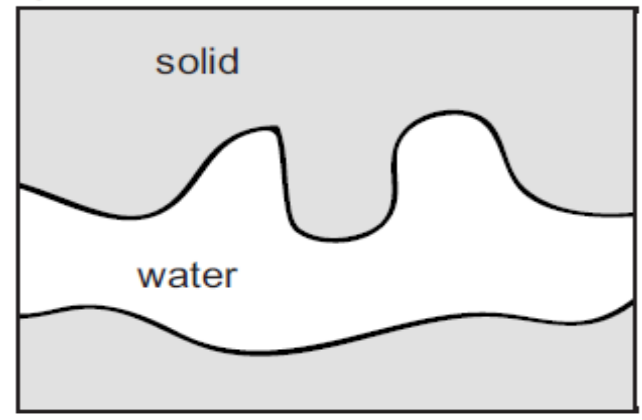

Figure 3.8: Three types of porous materials characterised by different polarization mechanisms. Type A corresponds to colloidal suspensions for which four polarization mechanisms coexist (polarization of the Stern and diffuse layers, membrane polarization, Maxwell-Wagner polarization). For dense granular media (Type B), the diffuse layer is continuous and does not polarize. In case of type $C$, the Stern and diffuse layers are continuous at the scale of representative elementary volume and therefor do not polarize. Only the membrane polarization and the Maxwell-Wagner polarization coexist and are influenced by the electrical conductivity in the electrical double layer (Revil and Cosenza, 2010). 


\subsection{Spectral Induced Polarization a mechanistic approach}

Revil et al., (2012b) give a summary description of the developments so far in terms of understanding the polarization phenomenon in porous media. The approach to understanding the IP mechanism is as follows. First, we look at the behaviour of a single grain in an alternating electric field and try to predict the outcome. Then, in the next step, an up scaling methodology is used to predict the result for bulk materials. In this approach the two steps are considered independent of each other. Two potential tools for the upscaling are a volume averaging approach, and a differential effective medium approach.

Attempts to describe the IP phenomenon in terms of polarization of the electric charges around a mineral grain begin with Schwarz (1962). Many people have since worked on this and proposed various models. The main discussion is around the nature of polarization of the electrical double layer. The focal point of these discussion is where the polarization takes place; whether it is only in the Stern layer or diffuse layer or if both of them polarize. Additionally, does the electrochemical environment and/or the pore size have an influence on the polarization or relative contribution from these two layers? Important contributions were made by Schurr (1964), Dukhin and Shilov (1974), Fixman (1980), Razilov and Dukhin (1995) and Zukoski and Saville (1986 a, b).

The work of Leroy et al. (2008), Revil and Florsch (2010), Schmutz et al. (2010), Vaudelet at al. (2011 a, b) and Revil and Skold (2011) favour the polarization of the Stern layer as the major source of the IP in the frequency range of $1 \mathrm{mHz}$ to $100 \mathrm{~Hz}$. These works resulted in the development of a mechanistic model that explains the SIP response as a result of Stern layer polarization and thereby relates the grain size to the Cole-Cole relaxation time constant. The Cole-Cole relaxation time constant is one of the parameters used to explain a SIP spectra. However, recently Revil et al. (2012) suggest that it is the characteristic pore size that controls the SIP response rather than the grain size of the porous medium. In this section an attempt is made to understand and explain the IP mechanism in terms of the polarization of the Stern layer and it is considered that other mechanisms are not significant in the frequency range of $1 \mathrm{mHz}$ to $1 \mathrm{kHz}$. The rationale is that in the case of unconsolidated sediments that are made of well-sorted grains the characteristic length scale that controls both the SIP response and the 
hydraulic conductivity of the porous medium is the same; irrespective of whether it is due to the grain size or the pore size/ pore throat size.

Revil and Florsch(2010) argue that the electrical conductivity of a porous medium (e. g. rock) consisting of grains with an electrical double layer as described above can be represented as

$\sigma_{0}=\frac{1}{F}\left(\sigma_{f}+\beta_{s} Q_{v}\right)$

where $\sigma_{0}$ is the DC electrical conductivity of the porous medium, $\sigma_{f}$ is the conductivity of the pore water, $\beta_{S}$ is the apparent mobility of counter ions, $Q_{v}$ is the total charge density of counter ions per pore volume and $F$ is the electrical formation factor of the medium.

In SIP we are interested in the variation of complex conductivity of the porous medium with frequency. A complex surface conductivity model is given by de Lima\& Sharma (1992); Leroy et al. (2008); Leroy and Revil (2009) and Revil and Florsch (2010) as

$\sigma_{S}=\frac{4}{d_{o}}\left(\Sigma^{d}+\Sigma^{S}\right)-\frac{4}{d_{0}} \frac{\Sigma^{S}}{1+i \omega \tau_{C C_{0}}}$

$\tau_{C C_{0}}=\frac{d_{0}^{2}}{8 D_{i}}$

where $\Sigma^{d}$ is the specific surface conductivity associated with the diffuse layer, $\Sigma^{s}$ is the specific surface conductivity associated with the Stern layer at high frequencies $(\omega \gg>$ $\left.\frac{1}{\tau_{C C_{0}}}\right)$, and $D_{i}$ is the diffusion coefficient of the counter ions in the fluid

Revil and Florsch (2010) explain both membrane polarisation and the Stern layer polarisation by analysing the behaviour of the electrical double layer under an applied electrical field. In the analysis, it is assumed that the polarization of the diffuse layer happens only if it does not overlap with the diffuse layer of the neighbouring grain. The current caused by the applied electric field flows through the electrolyte, and also through the electrical double layer surrounding the grain (Figure 3.7). In the electrical double layer, the current density is greater in the Stern layer as it contains most of the counter ions. In the electrolyte the current resulting from counterions and coions is proportional to their concentrations. As far as the current in the electrical double layer 
is concerned the higher concentration of counterions prevents the flow of coions through the electrical double layer. This leads to an accumulation of coions on one side of the grain, and thereby an increase in concentration of salt. The difference in concentration of salt levels is then balanced by a back diffusion through the pore space surrounding the mineral grain. This is nothing but membrane polarization.

The polarization of the Stern layer can be considered as the mechanism that gives rise to the frequency dependent variation of surface conductivity under the following assumptions. (1) The double layer is thin with respect to the radius of the grains. (2) The charges of the Stern layer can move only tangentially along the mineral surface. Backdiffusion in the Stern layer is responsible for the decrease of electrical conductivity with decrease in frequency. (3) The polarization of the diffuse layer is minimized by the fact that the diffuse layer is above a percolation threshold at the scale of a representative elementary volume. Therefore, there is no or only small deformation of the diffuse layer in response to an electric field as illustrated in Figure 3.7.

Given sufficient time the back-diffusion of counterions in the Stern layer can nullify the dipole moment around the mineral grain. The time taken for this is represented by the relaxation time constant $\tau_{C C_{0}}$. The relaxation time constant $\tau_{C C_{0}}$ is given by eqn (3.3) and is proportional to the square of the grain size. An equation for the variation of complex surface conductivity caused by the polarization of the Stern layer can be written as

$\sigma_{s}=\sigma_{s}^{\infty}+\frac{\sigma_{s}^{0}-\sigma_{s}^{\infty}}{1+i \omega \tau_{C C_{0}}}$

where

$\sigma_{s}^{0}=\frac{4}{d_{o}} \Sigma^{d}$

and

$\sigma_{s}^{\infty}=\frac{4}{d_{o}}\left(\Sigma^{d}+\Sigma^{s}\right)$

are the low frequency and high frequency asymptotic surface conductivities as can be seen from letting $\omega \rightarrow 0$ and $\omega \rightarrow \infty$ in equation (3.2). We can expect equation (3.4) to describe the variation of complex surface electrical conductivity of a porous medium only if the medium consists of identical grains. In reality all porous media will have a grain size distribution and hence a range of relaxation time constants rather than a single relaxation time constant. 
Revil and Florsch, (2010) suggest an appropriate expression to replace the single relaxation time with a distribution of relaxation times is

$\sigma_{s}=\sigma_{s}^{\infty}+\left(\sigma_{s}^{0}-\sigma_{s}^{\infty}\right) \int_{0}^{\infty} \frac{g\left(\tau_{C C}\right)}{1+i \omega \tau_{C C}} d \tau_{C C}$

where

$\int_{0}^{\infty} g\left(\tau_{C C}\right) d \tau_{C C}=1$

Equation (3.7) is a generalization of equation (3.4) and gives a distribution of relaxation times rather than a single relaxation time $\tau_{C C_{0}}$. This means that the both low and high frequency contributions to surface conductivities are also convolved by the grain size distribution. The next step is to replace the function $\int_{0}^{\infty} \frac{g\left(\tau_{C C}\right)}{1+i \omega \tau_{C C}} d \tau_{C C}$ that describes the distribution of relaxation times with a function that represents the distribution of grain sizes that in turn causes the distribution of relaxation times rather than a single relaxation time. Revil and Florsch, (2010) go on to show why Cole-Cole and log-normal probability distribution are successful in fitting the observed polarization spectra at low frequencies. If the distribution of relaxation times obeys a Cole-Cole distribution, then the surface conductivity is given by

$\sigma_{s}=\sigma_{S}^{\infty}+\left(\sigma_{s}^{0}-\sigma_{s}^{\infty}\right)\left[\frac{1}{1+\left(i \omega \tau_{C C}\right)^{c}}\right]$

where $c$ is the Cole-Cole exponent. The complex bulk conductivity of a porous medium $\left(\sigma^{*}\right)$ can be considered as the sum of ionic conductivity through the pore fluid and the complex surface conductivity. Revil and Florsch, (2010) suggest the following linear model.

$\sigma^{*}=\frac{1}{F}\left[\sigma_{f}+(F-1) \sigma_{s}\right]$

The overall conductivity model then becomes

$\sigma^{*}=\sigma_{\infty}+\frac{\sigma_{0}-\sigma_{\infty}}{1+\left(i \omega \tau_{C C}\right)^{c}}$

where

$\sigma_{0}=\frac{1}{F}\left[\sigma_{f}+(F-1) \sigma_{s}^{0}\right]$

$\sigma_{\infty}=\frac{1}{F}\left[\sigma_{f}+(F-1) \sigma_{s}^{\infty}\right]$

The above discussion gives a theoretical foundation for analysing the complex conductivity spectra in terms of a relaxation mechanism. In its simplest form, i.e. when all the mineral grains in the media are perfect spheres, the SIP spectra can be fitted by a Debye relaxation model. In reality, the grain size distribution, and the fact that probably none of the grains will be a perfect sphere, suggest that the experimental 
spectrum is going to be better described by a model that accounts for a distribution of relaxation times rather than a single relaxation time constant. This has led to the use of a number of empirical models to fit the experimental complex conductivity/resistivity spectra. All these models are based on the Cole-Cole dielectric dispersion model (Cole and Cole, 1941).

\subsection{SIP spectra models}

The purpose of analysis of the complex impedance spectra in this thesis is to estimate the so called SIP parameters which in turn can be used to predict hydraulic properties of the porous media. It can be seen from the literature that the lack of a single mechanistic model has led to the use of a wide variety of phenomenological models. Table 3.1 from Dias (2000) lists the commonly used SIP spectra models. This is not surprising because of the wide variety in the nature of the interfaces present in various porous media and their respective SIP spectra which show tremendous variation from one another. It is interesting to note that certain phenomenological models fit a particular set of data well but fail to sufficiently fit all the data found in the literature. Also the relationship between the SIP parameters and bulk properties of the medium differs according to the phenomenological model used. This makes it difficult to make meaningful comparison of results in the literature. Some of most commonly used phenomenological models are discussed below.

\subsubsection{The Debye model}

The very first attempt to use a phenomenological model to explain a spectrum can be traced back to the work of Debye for explaining the variation with frequency of the dielectric constant of a polar liquid with frequency (Debye, 1929). Debye suggested the dispersion of dielectric constant can be described by the following equation

$\varepsilon^{*}=\varepsilon_{\infty}+\frac{\left(\varepsilon_{0}-\varepsilon_{\infty}\right)}{\left(1+i \omega \tau_{C C_{0}}\right)}$

where $\varepsilon^{*}$ is the complex dielectric conductivity, $\varepsilon_{\infty}$ and $\varepsilon_{0}$ are the limiting values (real) of $\varepsilon^{*}$ at very high and very low frequencies, $\tau_{C C_{0}}$ is the relaxation time and $\omega$ is the angular frequency. The subscript CC is used to differentiate between the relaxation time used with two different models, the Cole-Cole model and the Pelton model.

Equation (3.4) is the equivalent of equation (3.14) in terms of electrical conductivity. As explained in the previous section, the Debye model may explain the SIP spectra of a 
porous medium consisting of identical grains. Some models consider the SIP spectra as a sum or product of a number of Debye relaxations.

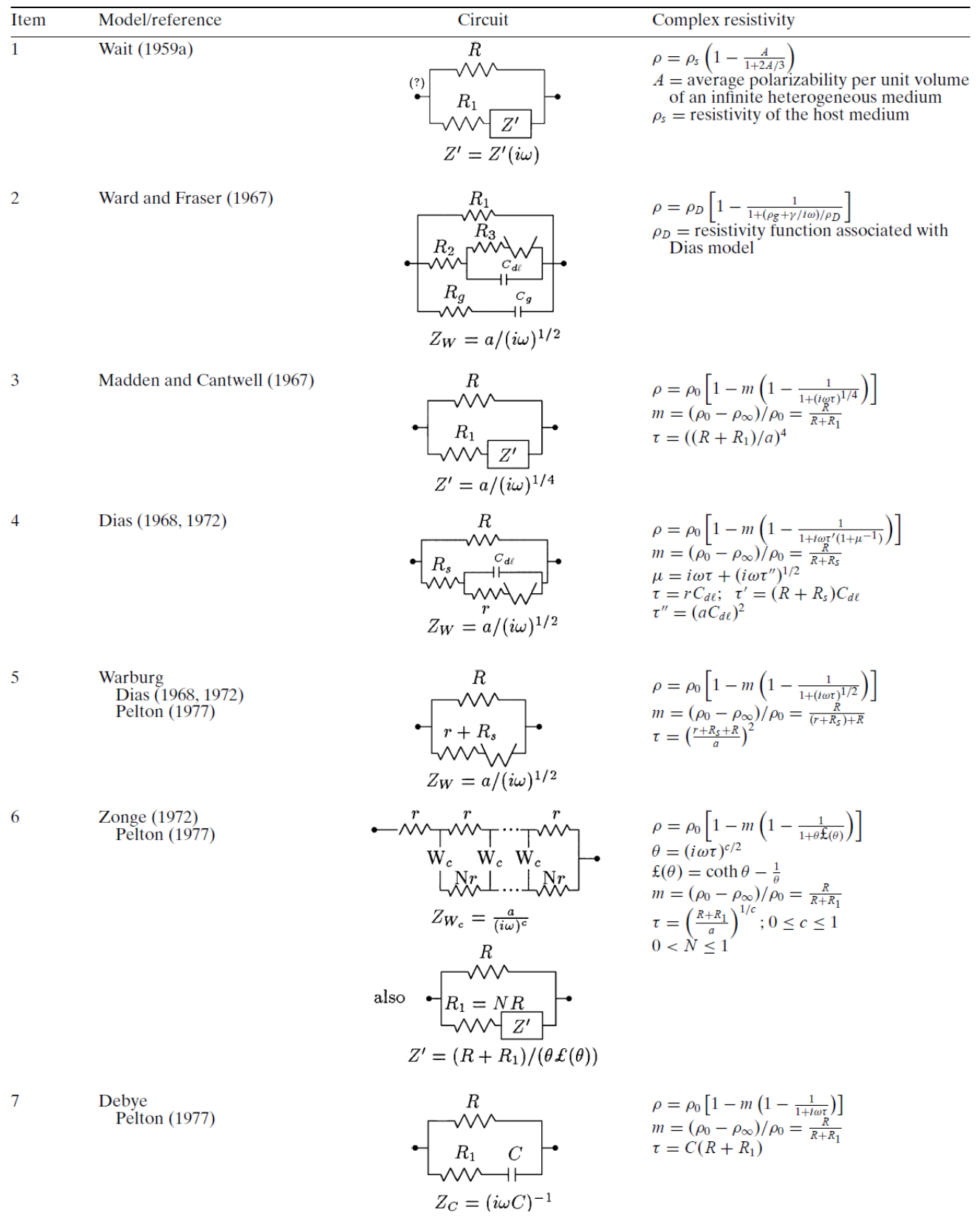

Table 3.1: Models, equivalent circuits and complex resistivities proposed for SIP (in chronological order). From Dias (2000).

\subsubsection{The Cole-Cole model}

Cole and Cole (1941) suggested modifications to the Debye model. They suggested the following empirical formula to describe the dielectric polarization.

$\varepsilon^{*}=\varepsilon_{\infty}+\frac{\left(\varepsilon_{0}-\varepsilon_{\infty}\right)}{\left[1+\left(i \omega \tau_{C C_{0}}\right)^{(1-\alpha)}\right]}$

Equation (3.15) differs from equation (3.14) by an additional term $(1-\alpha)$ appearing as the power of the term $\left(i \omega \tau_{C C}\right)$. This is sometimes represented by $c$, commonly known 


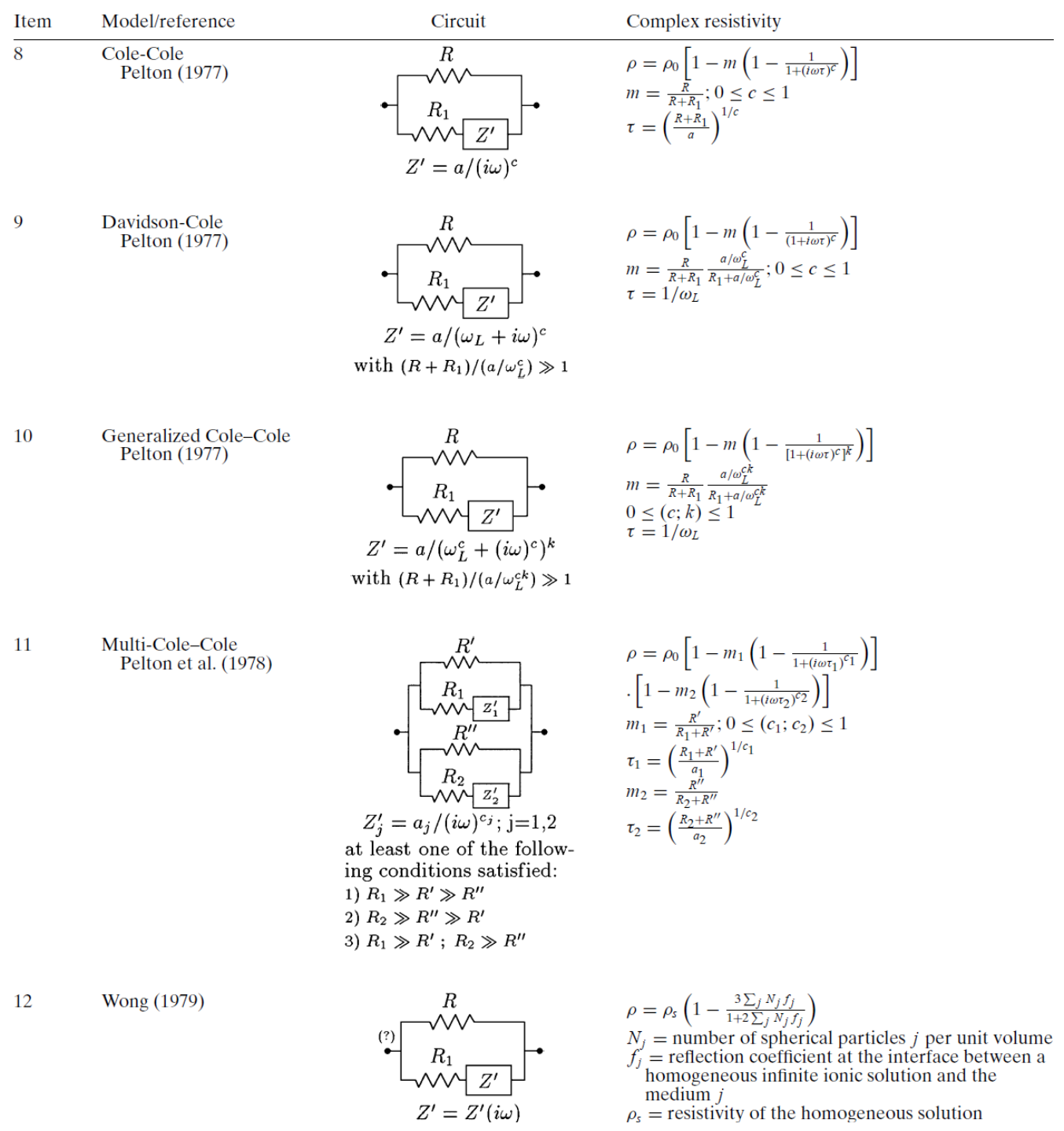

Table 3.1 continued.

as the Cole-Cole exponent. The introduction of the term $(1-\alpha)$ is mainly to account for the distribution of relaxation times. The Cole-Cole equation has been used to describe the complex electrical conductivity by substituting the complex dielectric constant with the complex electrical conductivity as follows (Tarasov and Titov, 2013)

$\sigma^{*}=\sigma_{\infty}+\frac{\sigma_{0}-\sigma_{\infty}}{1+\left(i \omega \tau_{C C}\right)^{c}}$

where $\sigma^{*}$ is the complex electrical conductivity, $\sigma_{\infty}$ and $\sigma_{0}$ are high frequency and low frequency values of electrical conductivity. Equation (3.16) can also be expressed as

$\sigma^{*}=\sigma_{0}\left\{1+\frac{M}{1-M}\left(1-\frac{1}{1+\left(i \omega \tau_{C C}\right)^{c}}\right)\right\}$

where $M$ is the chargeability given as

$M=\frac{\left(\sigma_{\infty}-\sigma_{0}\right)}{\sigma_{\infty}}$ 
It is common to express the SIP spectra in terms of complex resistivity and the Cole-Cole equation can also be written in terms of complex resistivity as follows

$\rho^{*}=\rho_{\infty}+\frac{\rho_{0}-\rho_{\infty}}{1+\left(i \omega \tau_{P M}\right)^{c}}$

where $\rho^{*}$ is the complex electrical resistivity, $\rho_{\infty}$, and $\rho_{0}$ are the high frequency and low frequency values of electrical resistivity. Here the subscript $P M$ stands for 'Pelton Model' Equation (3.17) which can also be expressed as

$\rho^{*}=\rho_{0}\left\{1-M\left(1-\frac{1}{\left(1+\left(i \omega \tau_{0}\right)^{c}\right)}\right)\right\}$

where $M$ is the chargeability given as

$M=\frac{\left(\rho_{0}-\rho_{\infty}\right)}{\rho_{0}}$

Both equations (3.17) and (3.20) are used for fitting SIP spectra. But it should be noted that they are not equivalent (Tarasov and Titov, 2013). This is highlighted by the use of two different subscripts for the relaxation time constant. Considering $\sigma^{*}$ as the reciprocal of $\rho^{*}$ rewriting equation (3.20) leads to the following equation

$\sigma^{*}=\sigma_{0}\left\{1+\frac{M}{1-M}\left(1-\frac{1}{1+\left(i \omega \tau_{P M}\right)^{c}(1-M)}\right)\right\}$

Equation (3.22) reduces to equation (3.17) if

$\tau_{P M}=\frac{\tau_{C C}}{(1-M)^{1 / c}}$

As there are two forms of the Cole-Cole model, the hereafter the subscript $C C$ or $P M$ is used with the relaxation time constant to indicate this difference.

\subsubsection{The Pelton model}

The most successful application of the so called Cole-Cole phenomenological model was by Pelton et al. (1978). They assumed that the dispersive nature of the resistivity is similar to that of the dielectric dispersion as explained by equation (3.15). Hence the Cole-Cole equation can be used to describe the SIP spectra by replacing the dielectric permittivity terms with resistivity terms, and they arrived at equation (3.20) as explained in section 3.5.2. It should be noted that there is no theoretical support for the use of the Cole-Cole model in explaining the dispersion of resistivity/conductivity. Still Pelton et al. (1978) were successful in analysing the spectra of mineralized porous rock.

\subsubsection{The Cole-Davidson model or Generalised Cole-Cole model}

Cole-Cole models like equation (3.16) fail when there is a considerable dispersion at lower and higher frequencies. Davidson and Cole (1951) modified the original Cole-Cole 
model, to account for these types of dispersion. If adopted like the Cole-Cole model the Cole-Davidson model can explain impedance spectra that are not fitted well with ColeCole models. A generalized model that can fit well on a wide range of experimental data suggested by Pelton et al., (1983) is

$\rho_{(\omega)}^{*}=\rho_{0}\left[1-M\left(1-\frac{1}{\left(1+\left(i \omega \tau_{P M_{G C C}}\right)^{c}\right)^{a}}\right)\right]$

Equation (3.24) is called the generalized Cole-Cole model. In equation (3.24) when $a=1$ it reduces to the Cole-Cole model. Again if $c=1$ then it becomes the Debye model.

\subsubsection{Cole-Cole model with multiple dispersion terms}

Further variations of Cole-Cole models, with more than one term for the relaxation process, have been successfully used for interpretation of measured data. In some models the resultant spectrum is considered as a product of the relaxation terms, while some consider it as the sum of the relaxation terms. Pelton et al. (1978) used the following equation with two relaxation terms to fit data with a higher frequency dispersion as shown in Figure 3.9

$Z_{(\omega)}=R_{0}\left\{1-\frac{1}{1+\left(i \omega \tau_{P M_{1}}\right)^{c_{1}}}\right\}\left\{\frac{1}{1+\left(i \omega \tau_{P M_{2}}\right)^{c_{2}}}\right\}$

Where $Z_{(\omega)}$ is the impedance $R_{0}$ is the DC electrical resistance $\tau_{P M_{1}}$ and $c_{1}$ are the relaxation time constant and Cole-Cole exponent for the relaxation taking place at the lower frequency while $\tau_{P M_{2}}$ and $c_{2}$ are the same for the relaxation at higher frequency.

Kemna (2000) used a modified form of the Cole-Cole equation. He considered that the SIP spectra may consist of one or more Cole-Cole dispersion terms. These additional terms can be SIP responses with multiple Cole-Cole relaxations, or can represent electromagnetic coupling terms

$\rho_{(\omega)}^{*}=\rho_{0}\left[1-\sum_{l=1}^{L} M_{l}\left(1-\frac{1}{1+\left(i \omega \tau_{P M_{l}}\right)^{c_{l}}}\right)\right]$

where $\rho_{(\omega)}^{*}$ is complex resistivity $\rho_{0}$ is DC resistivity, $M_{l}$ is chargeability, $\tau_{P M_{l}}$ is ColeCole relaxation time constant $c_{l}$ is the Cole-Cole exponent and $l$ is the number of ColeCole relaxations. 


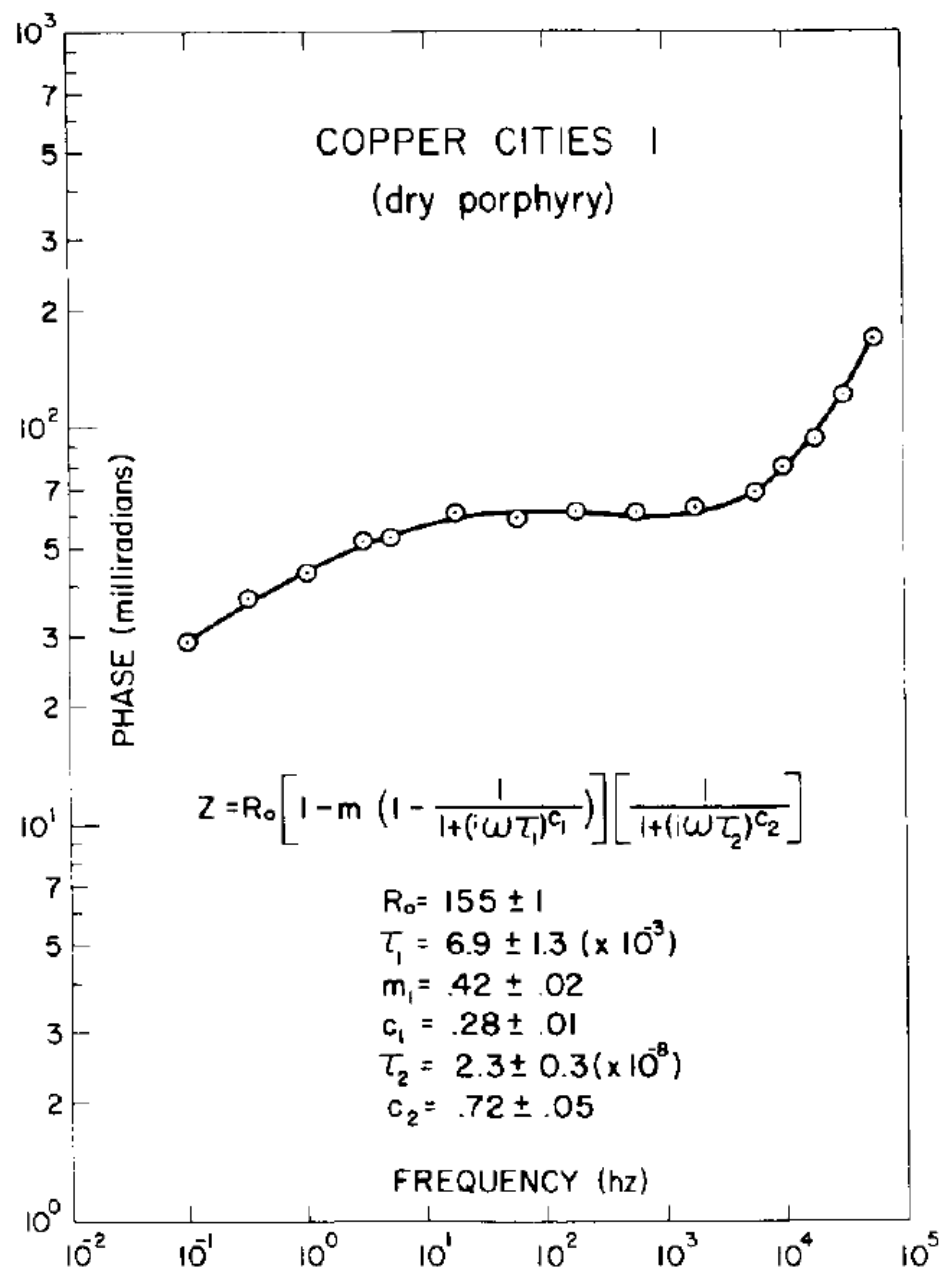

Figure 3.9: Phase angle spectra fitted with a Cole-Cole model that has two relaxation terms (Pelton et al., 1978).

\subsubsection{The Generalised Cole-Cole model with high-frequency capacitive element}

Kruschwitz et al., (2010) suggest that the high frequency dispersion can be very well fitted by adding a high-frequency capacitive element $\varepsilon_{\infty}$ in parallel with the generalized Cole-Cole relaxation model. They used the following equation

$$
\sigma_{(\omega)}^{*}=\frac{\sigma_{0}}{1-m\left(1-\left(\frac{1}{\left(1+i \omega\left(\tau_{P M_{G C C}}\right)^{c}\right)^{a}}\right)\right)}+i \omega \varepsilon_{\infty}
$$

\subsubsection{The Debye decomposition model.}

The fundamental mechanism of polarization of charges, whether it is in the Stern layer or in the diffuse layer, is described in terms of the Debye relaxation as described by equation (3.14). The presence of a range of such relaxation times may be a function of 
the variation in grain size and/or the fractal nature of the rough surface of grains that necessitates the use of a Cole-Cole model for the description of low frequency impedance spectra. So it can be argued that the resultant spectrum is a superposition of a large number of Debye relaxations. The Debye decomposition model was put forward by Nordsiek and Weller (2008). They consider both the spectra of resistivity amplitude and phase as a superposition of a number of different Debye spectra. These spectra are each characterised by a specific chargeability $M_{k}$ and relaxation time $\tau_{P M_{k}}$; considering that the Debye formula represents the frequency analogue of an exponential decay function in the time domain. According to Nordsiek and Weller (2008) the frequency dependant impedance spectrum is described by the equation

$Z_{(\omega)}=R_{0}\left(1-\sum_{k=1}^{n} M_{k}\left(1-\frac{1}{1+i \omega \tau_{P M_{k}}}\right)\right)$

The $n$ pairs of relaxation time $\tau_{P M_{k}}$ and chargeability $M_{k}$, and the value of DC resistance $R_{0}$, are the resulting parameters of this model. The DC resistance $R_{0}$ is approximated by extrapolating the impedance amplitude to lower frequencies. In an additional step, after the determination of all other parameters, the value of $R_{0}$ is adjusted using only the amplitude spectrum. In order to determine the parameters of this model, the impedance is normalized and then separated into real and imaginary parts and expressed in a matrix form. This over determined system of linear equations is solved under the constraint that no negative chargeability value $M_{k}$ is allowed. This can be easily implemented in MATLAB using the "Isqnonneg" function.

The result of Debye-decomposition analysis is expressed in terms of the following four integrating parameters

(1) The total chargeability $\sum M$ which sums up all chargeability values $M_{k}$ determined as solution from the system of linear equations

(2) The mean relaxation time $\overline{\tau_{P M}}$ describes the logarithmic average value of the relaxation times weighed by their chargeability. It is calculated using the formula

$\overline{\tau_{P M}}=\exp \left(\frac{\sum_{k} M_{k} \ln \tau_{P M_{k}}}{\sum_{k} M_{k}}\right)$

(3) Now use a non-uniformity parameter $U_{\tau_{P M}}$ defined in analogy to the degree of nonuniformity of a grain-size distribution curve and is calculated using the formula

$U_{\tau_{P M}}=\frac{\tau_{P M_{60}}}{\tau_{P M_{10}}}$ 
Expressions $\tau_{P M_{10}}$ and $\tau_{P M_{60}}$ mark those relaxation times whereby in a cumulative curve $10 \%$ and $60 \%$ of the total chargeability is reached. The parameter $U_{\tau_{P M}}$ characterises the width of the relaxation time distribution which reflects the different scale lengths of structures involved in the relaxation process.

\subsection{Conclusion}

This chapter explains five main mechanisms that give rise to the SIP response and various models used to explain the observed SIP spectra. Among these five mechanisms the predominant mechanism depends on the nature of the porous medium. The mechanistic approach by Revil and Florsch (2010) is able to give an explanation of why the Cole-Cole model is able to fit the observed SIP spectra and also tries to relate the distribution of relaxation times to the distribution of grain sizes in the medium. The presence and ability of various SIP spectra models is an indication of the complexity of the phenomenon of Spectral Induced Polarization. There is no single model yet that is able to completely explain the SIP spectra. 


\section{Instrumentation and Experimental Setup}

\subsection{Introduction}

This chapter gives the details of the instrumentation, experimental setup, and the procedures adopted for sample preparation and measurement of hydraulic conductivity, porosity, formation factor and pore fluid electrical conductivity. The details include the working and the design of the custom made impedance spectrometer, the setup of the permeameter used to measure hydraulic conductivity, and the sample holder design. Good quality SIP measurements demand taking care of a number of issues. Kemna et al., (2012) identify some of the issues involved in the study of unconsolidated sediments using SIP and suggest the best practices. Both the procedure for the measurements and the experimental set up used in this work continued to evolve throughout the project. Excellent quality, reproducible measurements of unconsolidated sediments are now possible.

\subsection{Operation and construction of the impedance spectrometer}

The SIP measurements on unconsolidated sediments involve measuring the impedance at a number of frequencies between $1 \mathrm{mHz}$ and $1 \mathrm{kHz}$. The impedance is measured in the form of the modulus of impedance and phase. For further interpretation the measured impedance can be expressed either in terms of complex resistivity or complex conductivity. The impedance measurements were carried out using a custom made impedance spectrometer. The impedance spectrometer was originally developed for insitu measurements of sea ice (Ingham et al., 2012). Figure 4.1 shows the block diagram and Figure 4.2 a photograph of the impedance spectrometer.

Some major changes were carried out to make the original instrument suitable for SIP measurements. They were: (1) Changing the signal generator to achieve low frequency sine wave signals; (2) Changes to the method of signal analysis to increase the accuracy of phase measurements; and, (3) Changes to the user interface and a number of other requirements like DC resistivity measurement and temperature measurement of the sample. This continued improvement of the software made the measurements more accurate and the software easier to use. The impedance spectrometer consists of a data acquisition device, a microcontroller, sine-wave generator, amplifier, electrode 
selection multiplexers, current control relays, electrodes, and the PC with control software. These are explained in detail below.

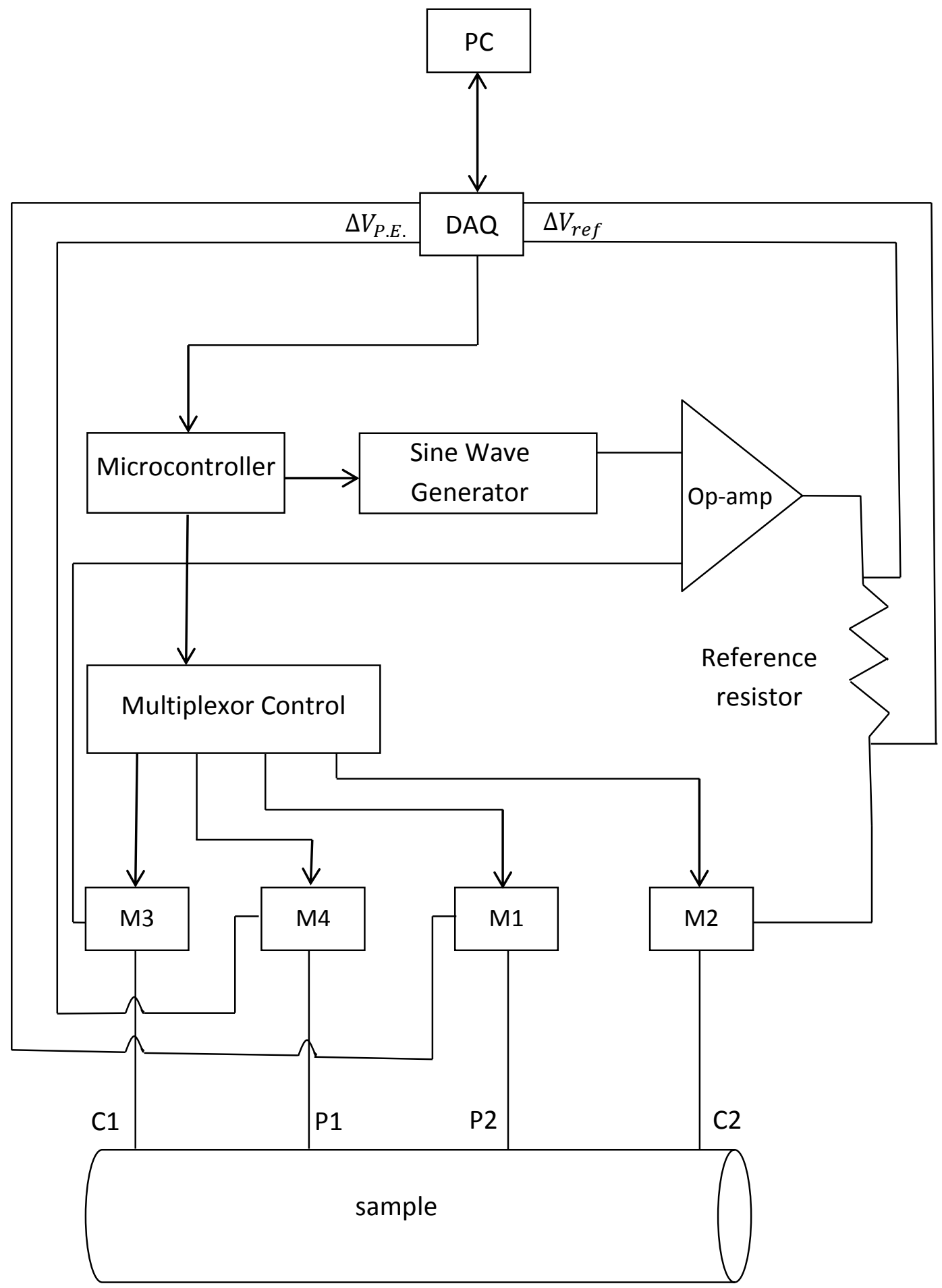

Figure 4.1: Block diagram of the impedance spectrometer used for SIP measurements. See text for definition of symbols. 


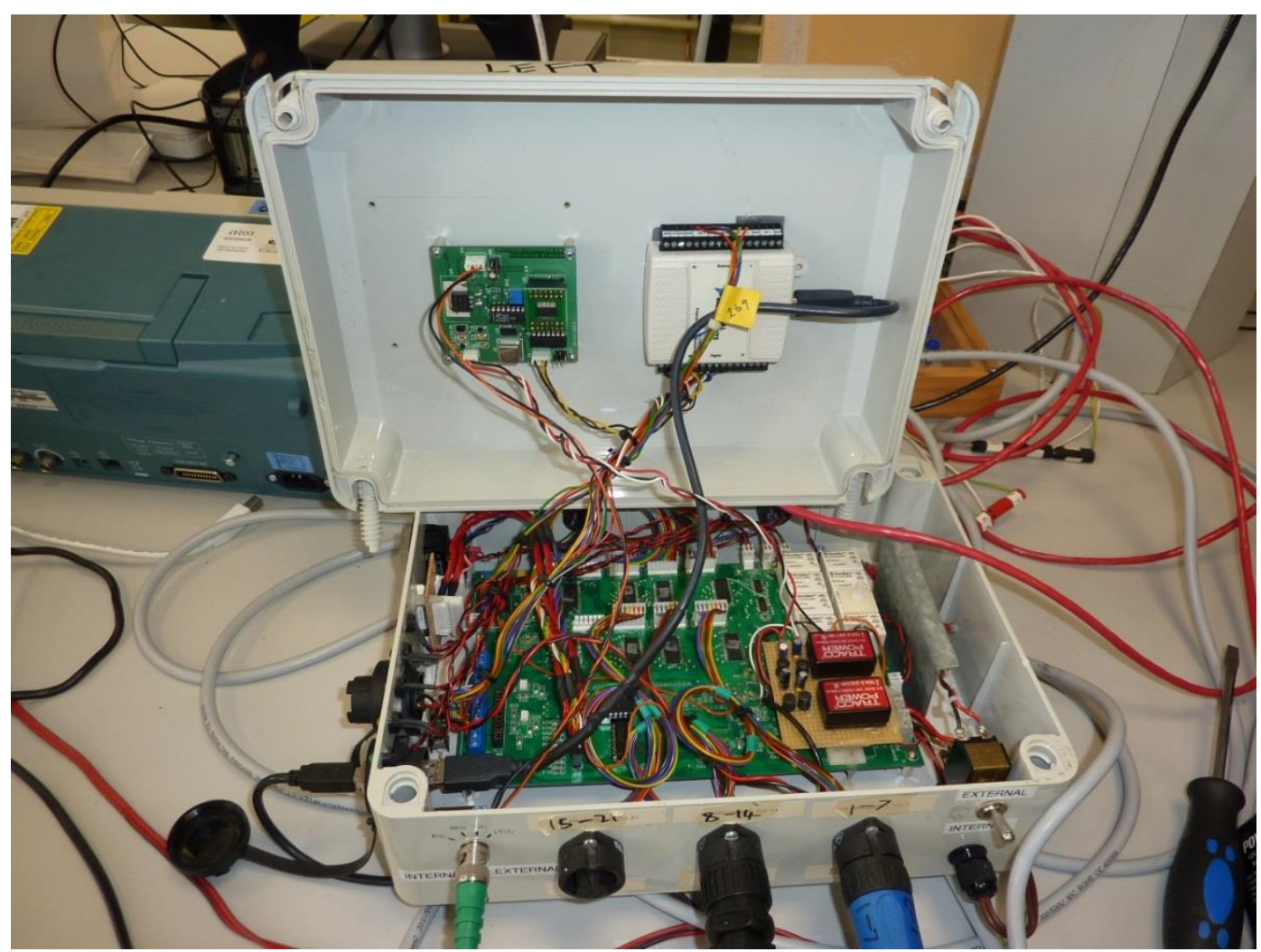

Figure 4.2: Impedance spectrometer used for SIP measurements.

\subsubsection{The data acquisition device - NI-USB-6009.}

The data acquisition (DAQ) device, NI-USB-6009 from National Instruments, plays a major role in the functioning of the impedance spectrometer. The DAQ device serves two functions: (1) It acts as the intermediary for all the communications between the PC and the microcontroller on board the impedance spectrometer; (2) It records voltage signals across the sample under study and across the reference resistance which are then used to determine the impedance. The DAQ device used in the impedance spectrometer is an NI-USB-6009.

The NI-USB-6009 is a low cost multifunction DAQ device ideal for data logging operations. It has 8 analog input channels with 13/14 bit resolution for single ended and differential inputs respectively. The maximum sampling rate is 48,000 Samples/s (S/s). This maximum sampling rate is divided among channels that are in use at any instant. This means that the maximum sampling rate of each channel will be $48 \mathrm{k} \mathrm{S} / \mathrm{s}$ divided by number of channels being used. They can be used as maximum of 8 single ended inputs or 4 differential inputs or a combination of single ended and differential inputs. The input voltage range for single ended operations is $\pm 10 \mathrm{~V}$. For differential input operation the input voltage ranges are $\pm 20 \mathrm{~V}, \pm 10 \mathrm{~V}, \pm 5 \mathrm{~V}, \pm 4 \mathrm{~V}, \pm 2.5 \mathrm{~V}, \pm 2 \mathrm{~V}, \pm 1.25 \mathrm{~V}$ and $\pm 1 \mathrm{~V}$. The 
DAQ comes with ready to run data logging software and NI-DAQmx Base measurement services driver software for programming the device in LabVIEW or C.

\subsubsection{The microcontroller - ATTiny84.}

The various units like the DDS sine-wave generator, current control relays and multiplexers are controlled using the on board microcontroller ATTiny84, from Atmel Corporation. Communication between the microcontroller and the controlling software is established through the digital outputs of the NI-USB-6009. These communications include the signals for selecting the desired current and the correct combination of potential and current electrodes.

\subsubsection{The signal generators.}

The signal used in the measurement of impedance is a sine wave. The original impedance meter was designed to generate 13 discrete frequencies between $10 \mathrm{~Hz}$ and $100 \mathrm{kHz}$, which covered the range of frequencies of interest in the case of sea ice studies. For good quality spectra of unconsolidated samples the required frequency range is 1 $\mathrm{mHz}$ to $1 \mathrm{kHz}$ with at least 10 logarithmically separated frequencies per decade. This necessitated finding a suitable replacement for the sine wave generator. During the course of this project two different sources were used for generating the sine wave.

\subsubsection{The Tektronix AFG 3102}

The low frequency signals were first achieved using a Tektronix AFG 3102 signal generator. The availability of a USB interface made the interfacing of the generator straightforward. Necessary changes were made to the software to control the Tektronix generator through the USB interface. The updated software enables the selection of any frequency between $1 \mu \mathrm{Hz}$ to $100 \mathrm{MHz}$ with a resolution of $1 \mu \mathrm{Hz}$. It is also possible to select either a sine wave or a square wave. The $1 \mu \mathrm{Hz}$ resolution of the signal generator is very helpful in optimizing the number of frequencies per decade for the complex resistivity spectrum. Figure 4.3 shows the Tektronix AFG 3102 function generator.

\subsubsection{The custom made signal generator with direct digital synthesizer}

At a later time during the project a new custom made sine wave generator was introduced. The main reason for this was that the bulky Tektronix AFG 3102 is 


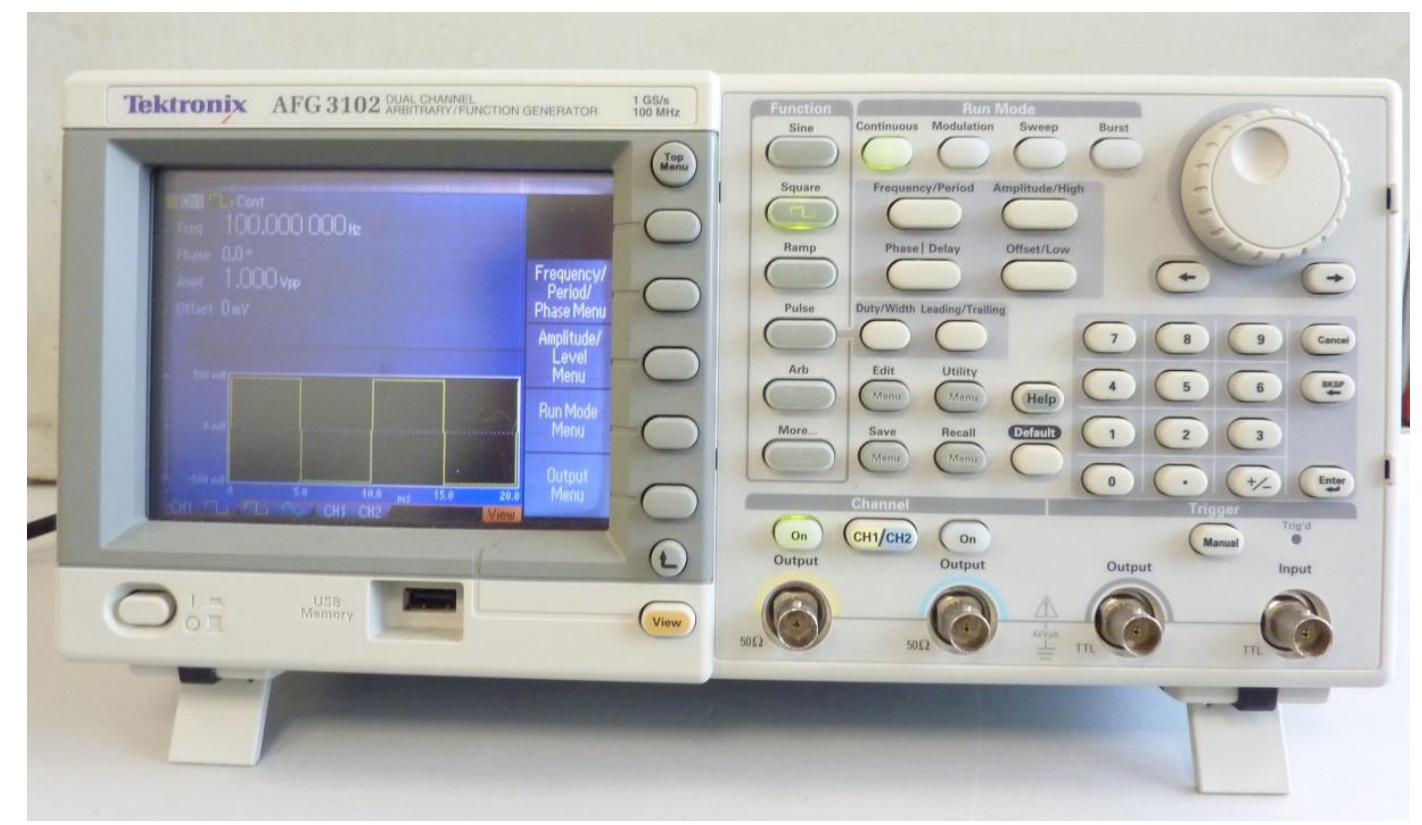

Figure 4.3: Tektronix AFG 3102 used initially for generating low frequency signals.

inconvenient for field measurements. It also consumes a large amount of power which is not a desirable feature when day-long field measurements are made in a remote area using battery power. A direct digital synthesizer (DDS) based sine wave generator is very compact and could be easily fixed inside the existing chassis of the impedance spectrometer. The power consumption of this new generator is about $1 \mathrm{~W}$ while that of the Tektronix AFG 3102 is $35 \mathrm{~W}$. However, the resolution of this new signal generator is $1.164 \mathrm{mHz}$ and the upper limit is $5 \mathrm{MHz}$, which has the effect of reducing the number of possible frequencies in the decade $1 \mathrm{mHz}$ to $10 \mathrm{mHz}$ from 10 to 7 . As the compactness of soil in locations where field measurements are generally made means that the range of frequencies at which relaxation occurs is much higher, this is unlikely to be a significant impediment. It does not affect the measured complex spectrum and the overall accuracy of the SIP parameters. However, the Tektronix AFG 3102 was used for all the laboratory measurements as it has better resolution, and neither size nor power consumption is an impediment for laboratory measurements.

The heart of the new signal generator is the AD9851 Direct Digital Synthesizer from Analog Devices. When referenced to an accurate clock source, the AD9851 generates a stable frequency and phase programmable digitized analog output sine wave. The frequency of the output from the DDS synthesiser is controlled by a 32-bit frequency 
'tuning-word'. The relationship between the output frequency, system clock, and the 'tuning-word' of the AD9851 is determined by the expression:

$f_{\text {out }}=\frac{(\Delta \text { Phase X System Clock })}{2^{32}}$

where

$\Delta$ Phase $=$ decimal value of 32-bit frequency tuning word

System Clock $=$ direct input reference clock (in $\mathrm{MHz})$

$f_{\text {out }}=$ frequency of the output signal in $\mathrm{MHz}$.

\subsubsection{The operational amplifier}

The sine wave signal generated by the Direct Digital Synthesizer is amplified in the next stage. The amplification is performed using an OPA827 JFET operational amplifier. After the amplification stage the resulting signal is $12 V_{p p}$ and can deliver up to a maximum of $20 m A_{p p}$.

\subsubsection{Electrode selection multiplexers}

A number of multiplexers are used to select the required pair of current and potential electrodes from among the available 21 pairs of electrodes. The multiplexor is the 16Ch/Dual 8-Ch CMOS Analog Multiplexer DG406. This is a 16 channel single-ended analog multiplexer designed to connect one of sixteen inputs to a common output as determined by a 4-bit binary address.

The combination of four multiplexers in the impedance spectrometer has the ability to connect 21 different pairs of electrodes. Any two of these 21 electrode pairs can be selected as a current or potential electrodes. The purpose of the multiplexer was to automatically scan through an array of electrodes for measurements on sea ice. This is not a required feature for SIP measurements for this project, but the multiplexers do give the impedance meter the ability to make measurements for complex resistivity tomography.

\subsubsection{Current control relays}

The microcontroller drives a number of relays to change the current through the sample holder. The available options of currents are $20 m A_{p p}, 10 m A_{p p}, 5 m A_{p p}, 2 m A_{p p}, 1 m A_{p p}$, $500 \mu A_{p p}, 200 \mu A_{p p}$ and $100 \mu A_{p p}$. The ability to change the current is important for two 
reasons. The first is to obtain a sufficiently high voltage signal, which is necessary to maintain the phase resolution. This is particularly true in the case of a sample with low impedance. The second reason is that the current has to be sufficiently low in the case of high impedance samples as the maximum available voltage of the signal is only $12 \mathrm{~V}_{\mathrm{pp}}$. This is a crucial feature because during salinity dependency the impedance of the samples varies from a few ohms to more than $10^{5} \Omega$ studies where the electrical conductivity varies from $5 \mu \mathrm{Scm}^{-1}$ to $1000 \mu \mathrm{Scm}^{-1}$ or more.

\subsubsection{The electrodes}

The potential electrodes are rings made of stainless steel. The current electrodes are either $15 \mu \mathrm{m}$ stainless steel mesh or $50 \mu \mathrm{m}$ brass mesh. Electrodes are explained in detail in section 4.5.1.

\subsubsection{PC with interface software.}

The measurements using the impedance spectrometer are controlled from the PC using custom made interface software. The software is developed in LabVIEW. A screen shot of the interface software is shown in Figure 4.4. The software provides an option to load a frequency configuration file that contains the values of frequencies at which the impedance is to be measured, and the sampling rate and number of samples for each of these frequencies. Depending on the resistance of the sample, there is an upper limit for the current that the impedance spectrometer can drive through the sample. Table 4.1 gives the maximum value of sample resistance corresponding to the 8 different currents available. The main factor that determines the resistance of the sample is the pore water electrical conductivity. If the impedance spectrometer cannot drive the selected current, then it will display a message of overload. Once an overload is detected, it either automatically steps down to the next current, or waits for the user to manually change the current; depending upon which option was selected by the user. After measurement at each frequency the software analyses the signal and displays the detected frequency of the signal, and the impedance in amplitude and phase form. A graph of the voltages across the reference resistor and the sample is provided. There is an option to save the raw signals for further analysis later. With an additional NI-USB- 
6009 and thermocouple the temperature of sample can be recorded for monitoring purposes.

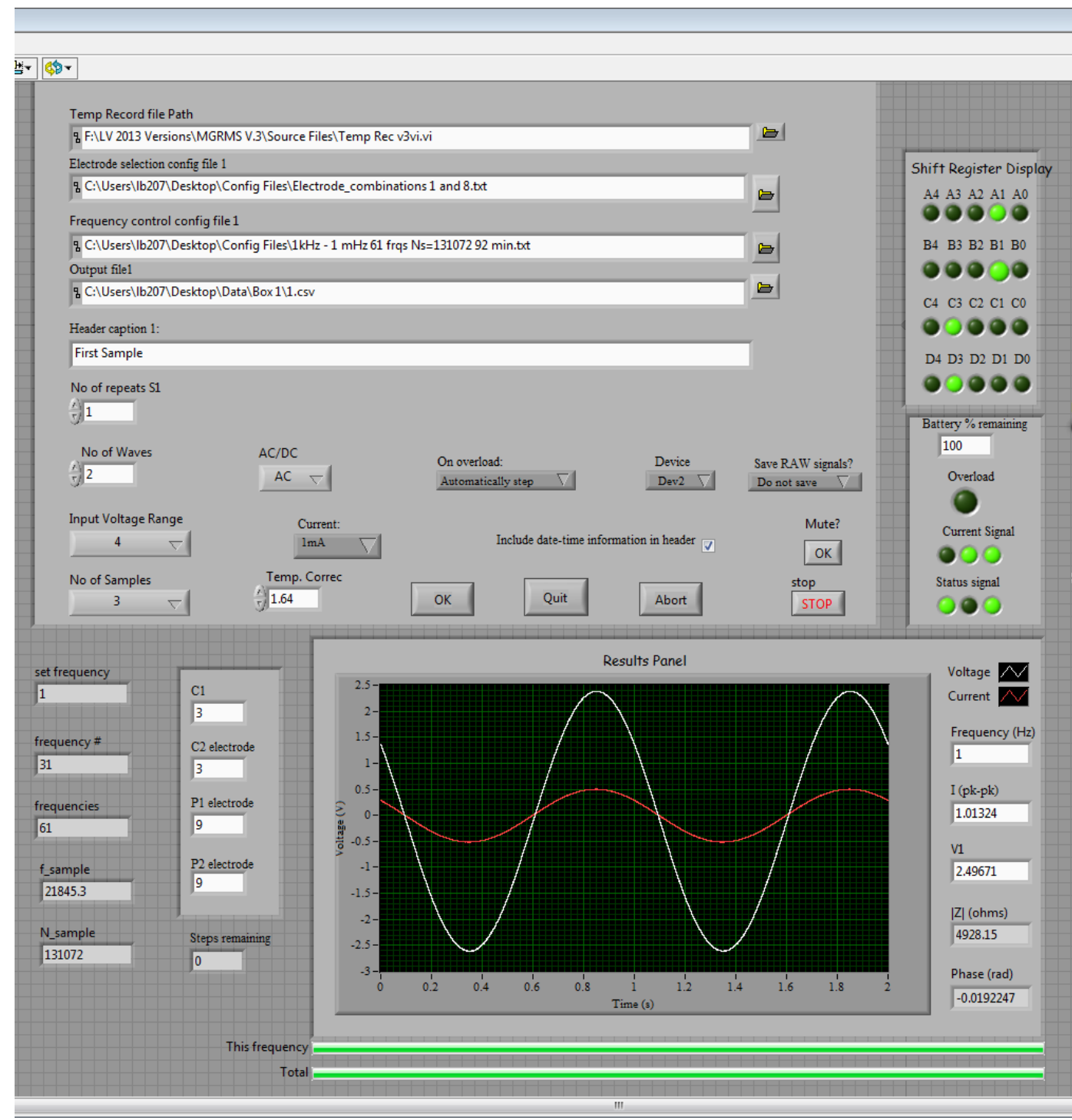

Figure 4.4: A screen shot of the front panel of the LabVIEW control software

\subsection{Measurement of DC resistance}

The electrical formation factor of the sample is calculated from the DC resistance of the sample measured at different values of pore water electrical conductivity. The DC resistivity measurements use a square wave instead of the sine wave that is used for the SIP spectra measurements. This change from sine wave to square wave is easy as the Tektronix AFG 3102 also generates square waves. The electrical conductivity of pore water used for DC resistivity measurements is in the range of $20 \mu \mathrm{Scm}^{-1}$ to $500 \mu \mathrm{Scm}^{-1}$. The IP effect exhibited by the samples increases with decrease in electrical conductivity; hence a square wave of sufficiently long period has to be used. Figure 4.5 shows the IP 
effect displayed by a sand sample. This IP effect, which is significant at low electrical conductivities of pore water, necessitates the use of a sufficiently long period of the square wave; otherwise it may lead to the underestimation of DC resistivity. The time period of the square wave used to determine the DC resistivity is $128 \mathrm{~s}$.

\begin{tabular}{|c|c|}
\hline $\begin{array}{l}\text { Current } \\
\text { in } \mathrm{mA}_{\mathrm{pp}}\end{array}$ & $\begin{array}{l}\text { Maximum } \\
\text { resistance of } \\
\text { the sample in } \Omega\end{array}$ \\
\hline 20 & 100 \\
\hline 10 & 200 \\
\hline 5 & 400 \\
\hline 2 & 1000 \\
\hline 1 & 2000 \\
\hline 0.5 & 4000 \\
\hline 0.2 & 10000 \\
\hline 0.1 & 20000 \\
\hline
\end{tabular}

Table 4.1: Values of current that can be selected in the impedance spectrometer (Peak to peak) and the corresponding maximum value of load resistance.

\subsection{Measurement of impedance}

Like any other SIP device, the custom built impedance spectrometer also measures the complex resistivity as two parts. The first part is the modulus or amplitude of the

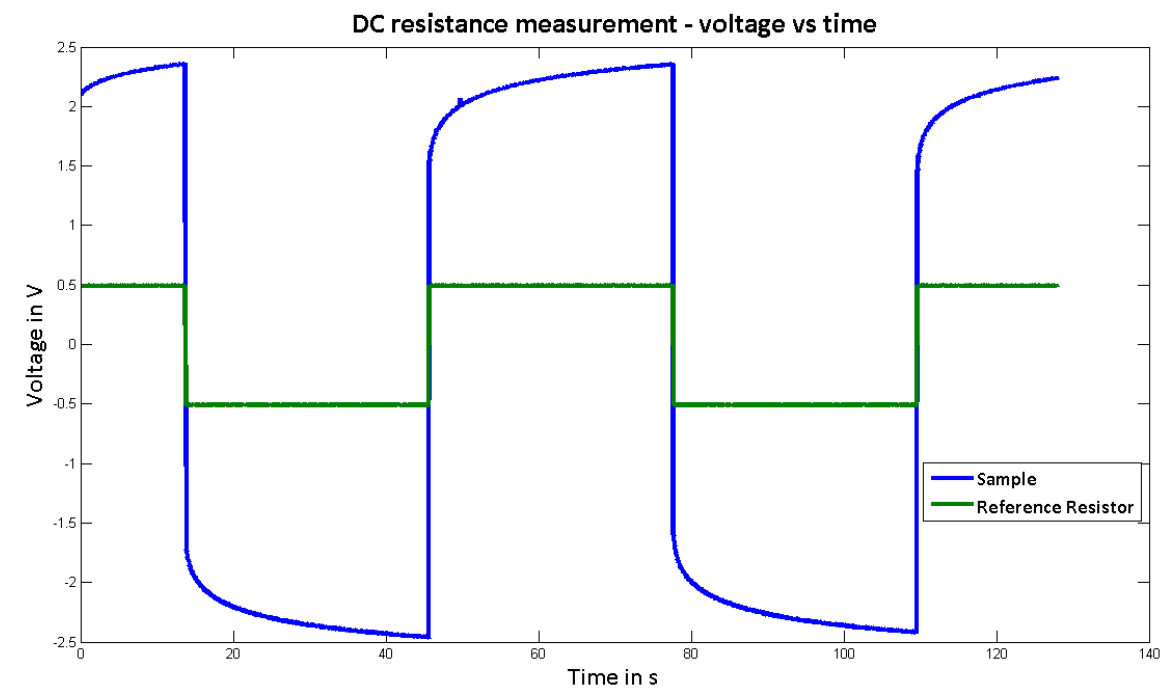

Figure 4.5: Typical IP effect displayed by a sand sample. 
impedance, and the second part is the phase difference between the applied current signal and the measured voltage between the potential electrodes. In order to measure the actual current supplied, the voltage across a reference resistor in series with the sample is measured.

The modulus of impedance is given by

$|Z|=\frac{\Delta V_{P . E .}}{\Delta V_{\text {ref }}} R_{\text {ref }}$

where $|Z|$ is the modulus of the impedance, $\Delta V_{P . E \text {. }}$ and $\Delta V_{\text {ref }}$ are respectively the voltage between the potential electrode and the voltage across the reference resistor respectively and $R_{r e f}$ is the value of standard resistance which is connected in series with the sample as shown in Figure 4.1.

The $D C$ resistance and impedance are determined by the analysis of the digitized voltage waveforms across the potential electrode and the reference resistor. The digitization of the voltage signals is performed by the Data Acquisition (DAQ) device NI-USB-6009. All DAQ devices digitize an analogue signal by measuring the signal with a certain frequency. The average number of samples measured per second is called the sampling rate or sampling frequency $\left(f_{s}\right)$. The digital to analogue conversion is done by the analogue-to-digital converters (ADC). The NI-USB-6009 has only one ADC and hence it is not a simultaneous sampling device. This means that it records the two voltage signals alternately, with a time delay dependent on the sampling rate or frequency of sampling. This alternation between the two channels that are connected to the voltage sources gives rise to an additional phase difference between the two recorded signals. This introduced phase difference depends on the sampling frequency. It is negligible at higher frequencies but becomes significant at low frequencies. This added phase difference caused by the non-simultaneous sampling of the voltage signals is corrected using a mathematical function implemented in the LabVIEW program. There a number of factors that affect the accuracy of the final SIP parameters estimated from the digitized voltage signals. These are discussed below 


\subsubsection{Number of frequencies in the SIP spectra.}

SIP parameters are estimated by fitting the measured spectra to the Cole-Cole model. In Cole-Cole modelling the accuracy of the estimates depends to some extent on the number of frequencies at which the spectra are recorded. Usually the frequencies are selected such that they are equally spaced in the logarithmic scale, so there can be 5,10 or 20 frequencies per decade. In this project measurements were made using the Tektronix AFG 3102 at ten logarithmically equally spaced frequencies per decade; with the frequency range of measurements covering six decades i.e. $1 \mathrm{mHz}-1 \mathrm{kHz}$. This means that the complex conductivity was measured at 61 different frequencies for most the measurements. Although increasing the number of frequencies at which measurements are made increases the accuracy of estimation of the SIP parameters, increasing the number of frequencies also increases the time required for completion of recording one complete spectrum. This is very significant as it is the frequencies below $1 \mathrm{~Hz}$ that are critical to this study. It is found that ten logarithmically equally spaced frequencies give a good compromise between accuracy and overall time. Table 4.2 gives the details of time duration for one set of measurement for a number of different frequencies in a decade.

\begin{tabular}{|c|c|c|c|}
\hline \multirow{2}{*}{$\begin{array}{c}\text { Number of } \\
\text { frequencies } \\
\text { per decade }\end{array}$} & \multicolumn{3}{|c|}{$\begin{array}{c}\text { Duration of Sampling in } \\
\text { hours }\end{array}$} \\
\cline { 2 - 4 } & 1 Cycle & 2 Cycles & 3 Cycles \\
\hline 5 & 1 & 2 & 3 \\
\hline 10 & 1.5 & 3 & 4.5 \\
\hline 20 & 2.7 & 5.4 & 8.1 \\
\hline
\end{tabular}

Table 4.2: The time taken for one complete set of measurement for three different numbers of frequencies per decade and three different durations of sample collection expressed in terms of the cycle of the respective frequencies

\subsubsection{The frequency of sampling and the number of samples.}

The amplitude and phase of the impedance is calculated using LabVIEW VIs. The abbreviation VI stands for Virtual Instrument, a term used in LabVIEW to refer to a program or function that does a specific task. The sampling rate or frequency of 
sampling $\left(f_{s}\right)$ and the number of samples collected $\left(N_{s}\right)$ affects the resolution of phase measurement. The resolution of the phase increases with increase in the values of $f_{s}$ and $N_{s}$; but both these values are limited by the capability the DAQ device. Appendix 1 gives the values of $f_{S}$ that were found to be optimum for measurements with NI-USB6009 when used with the custom made impedance spectrometer. The maximum possible value of $f_{s}$ with the NI-USB-6009 when two channels are being used is 24,000 samples per second $(S / s)$. As the highest frequency at which the impedance is measured is only $1000 \mathrm{~Hz}$ at each frequency a maximum sampling rate of $24,000 \mathrm{~S} / \mathrm{s}$ is much greater than the Nyquist rate. The Nyquist rate is the minimum sampling frequency required to avoid aliasing. The sampling frequencies listed in Appendix 1 give excellent phase resolution.

The phase resolution also improves with the duration of sample collection. For a given sampling rate, the duration of sample collection is determined by the number of samples collected $\left(\mathrm{N}_{s}\right)$. As the number of samples is increased at a given sampling rate the time required for the completion of measurement also increases. This means that there is a trade off between the phase resolution and the time taken to complete a set of SIP measurements.

Table 4.2 gives the time taken for measurements for different durations of sample collection, expressed in terms of the number of cycles. The number of samples collected is chosen based on two criteria; first, they are a power of 2; and, secondly the total time for collection of all these samples is equal to an integral multiple of the time period of the frequency at which the measurements are made. In other words, the samples are collected for an integral number of cycles. This makes sense as the estimation of phase is done by a Fast Fourier Transform.

\subsubsection{Testing of the impedance spectrometer with an RC circuit.}

An easy way to check the accuracy and resolution of the impedance spectrometer is to measure the impedance of an RC circuit. Figure 4.6 shows the diagram of the RC circuit used for the testing of the impedance spectrometer. Figures $4.7,4.8$ and 4.9 show the comparison between the theoretically predicted phase values of the RC circuit and the measured values of phase. The measurements were made for different number of samples as explained. It can be noted that there is slight deviation from theoretical 
values at very low frequencies when the duration of collection is only one cycle. The mismatch between the calculated and measured phase close to $1 \mathrm{kHz}$ is due to instrumentation related effects.

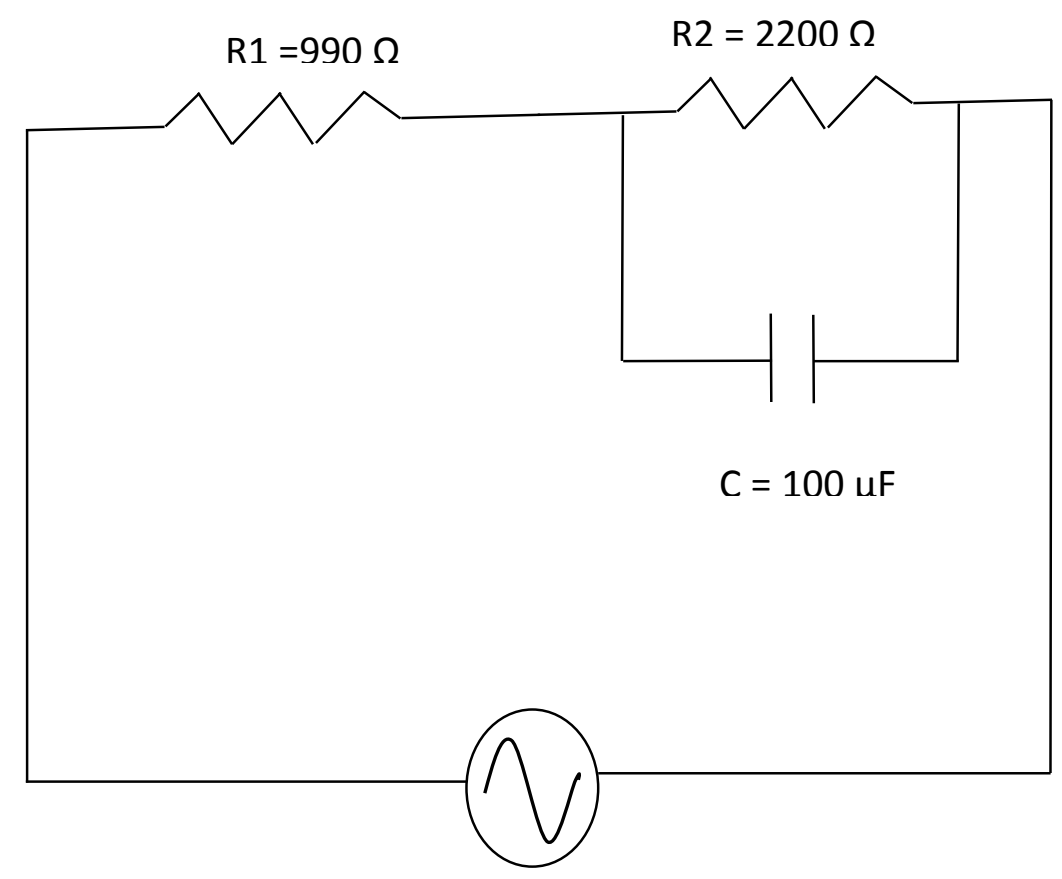

Figure 4.6: Circuit diagram of the RC circuit used to check measurement of phase by the impedance spectrometer.

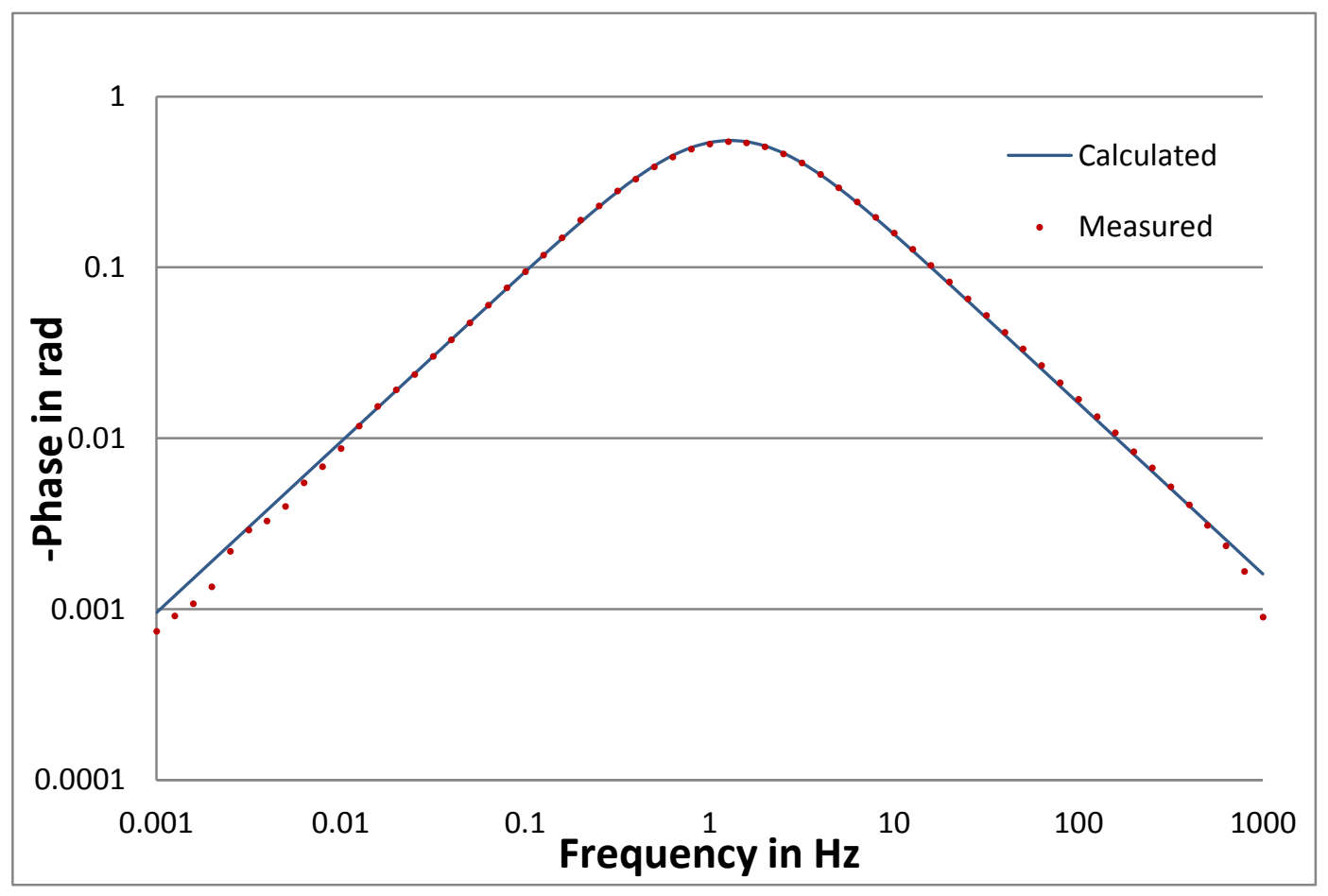

Figure 4.7: Calculated and measured phase for the circuit in Figure 4.6 for measurements made over 1 cylce at each frequency. 


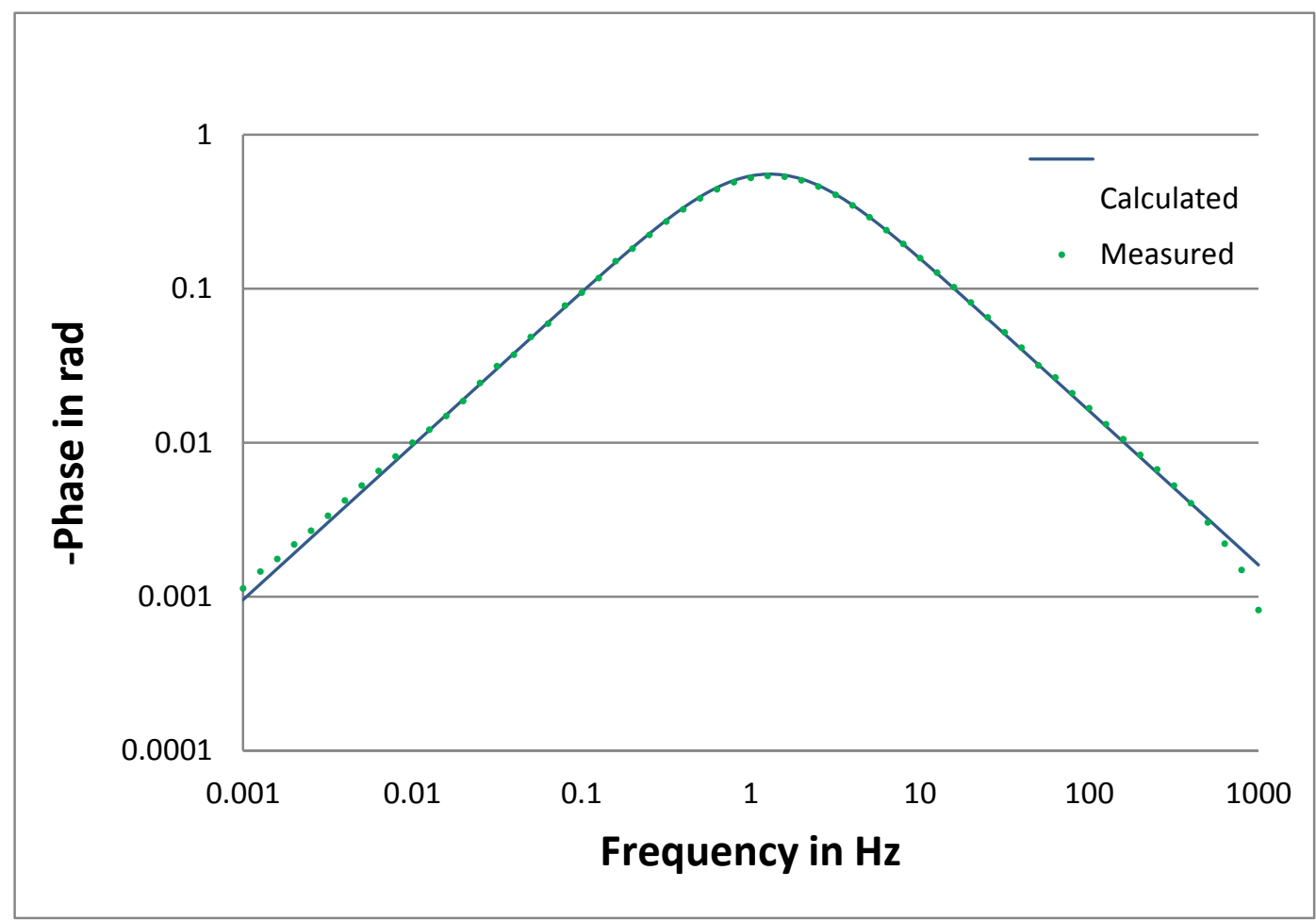

Figure 4.8: Calculated and measured phase for the circuit in Figure 4.6 for measurements made over 2 cycles at each frequency.

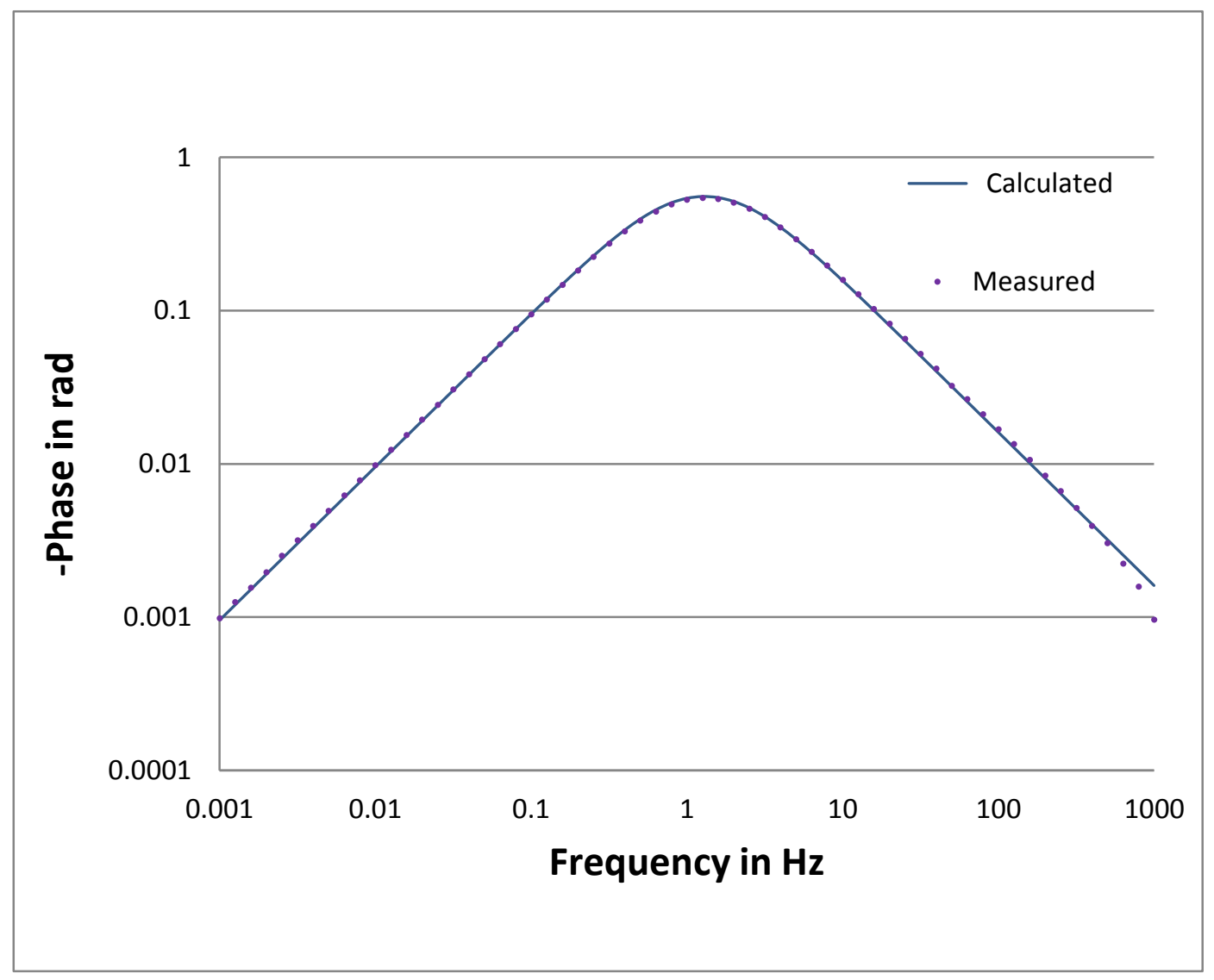

Figure 4.9: Calculated and measured phase for the circuit in Figure 4.6 for measurements made over 3 cycles at each frequency. 


\subsubsection{Testing of the impedance spectrometer with water of various electrical conductivities.}

The impedance of water depends on its electrical conductivity. As the electrical conductivity decreases the phase of the impedance of water increases (Lasne et al., 2008). Figure 4.10 shows the phase vs frequency plot of water at different electrical conductivities. Measurements were made at six different electrical conductivities in the range of $5 \mu \mathrm{Scm}^{-1}$ to $1000 \mu \mathrm{Scm}^{-1}$. The very small phases measured at low frequencies are an indication that there are no significant electrode polarization effects resulting from the use of stainless steel potential electrodes.

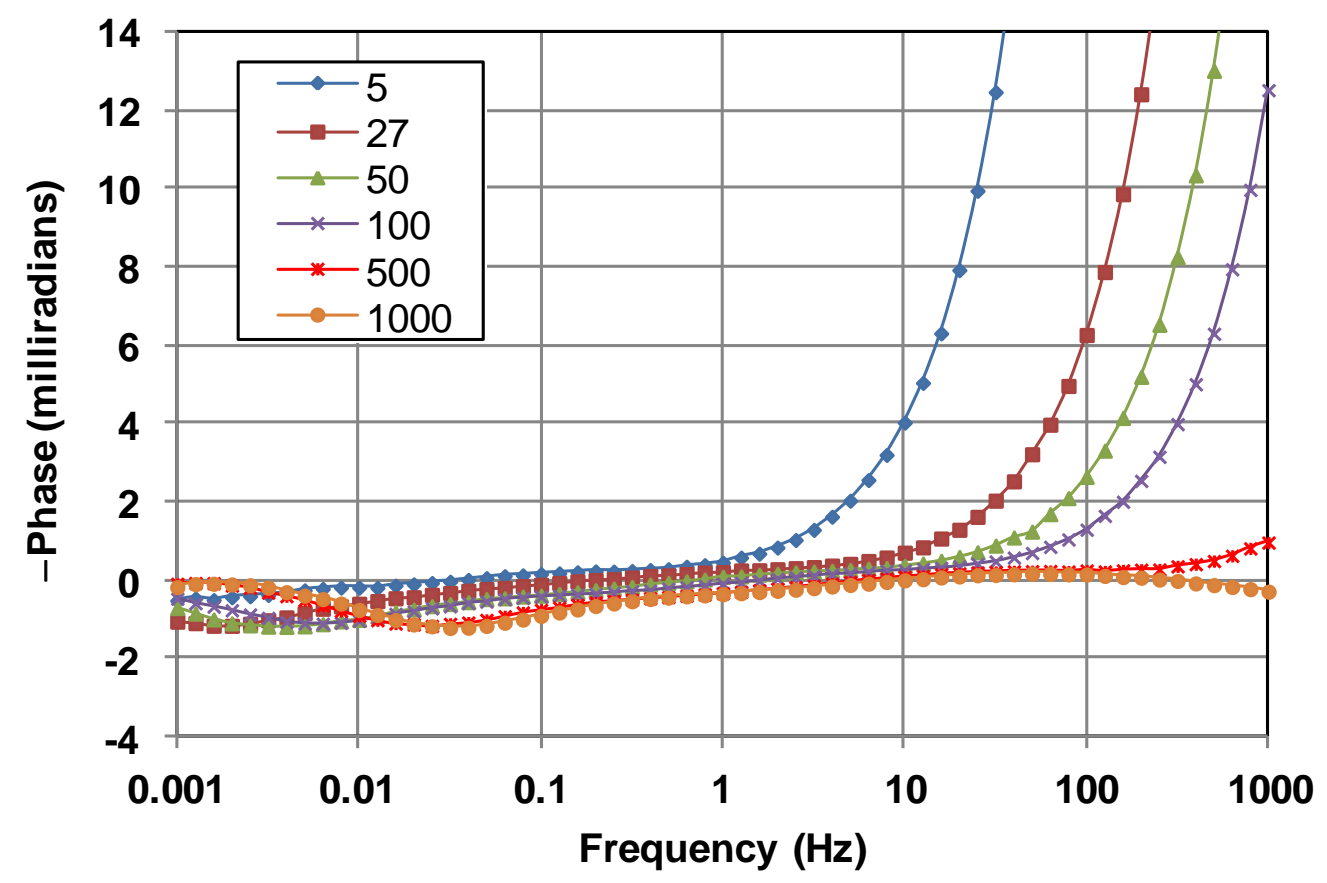

Figure 4.10: Measurements of phase made on water samples of different conductivities (in $\mu \mathrm{S} . \mathrm{cm}^{-1}$ ).

\subsubsection{Measurements on low polarizing sand}

Measurements on pure silica sand which has a very low polarizability are shown in Figure 4.11. This further confirms that spurious phase effects, resulting for example from electrode polarization, are minimal over the range of $1 \mathrm{mHz}$ to $1 \mathrm{~Hz}$. 


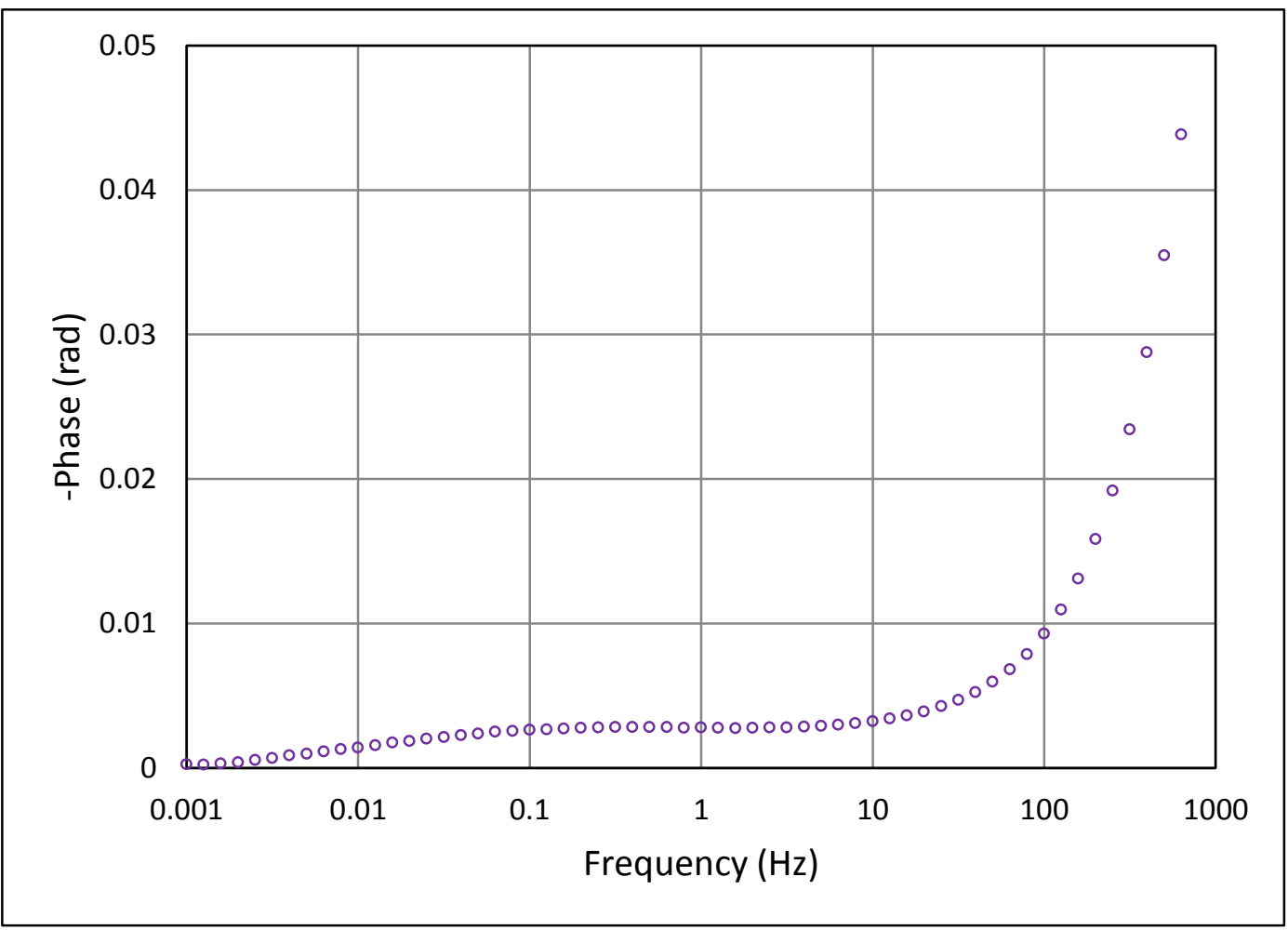

Figure: 4.11: SIP phase measurements made on pure silica sand

\subsection{Hydraulic conductivity measurements.}

Hydraulic conductivity, measured in $\mathrm{ms}^{-1}$, represents the ease with which water flows through a porous medium such as soil or rock. This ability of a porous material to allow water flow can also be expressed in terms of permeability. Permeability is an intrinsic property of the material from which the rate of flow of any fluid can be calculated from the knowledge of the dynamic viscosity and density of the fluid. The relationship between hydraulic conductivity and permeability is given below

$k=K \frac{\mu}{\rho g}$

where

$k$ is the permeability in $\mathrm{m}^{2}, K$ is the hydraulic conductivity in $\mathrm{ms}^{-1}, \mu$ is the dynamic viscosity of the fluid in $\mathrm{kgm}^{-1} \mathrm{~s}^{-1}, \rho$ is the density of the fluid in $\mathrm{kgm}^{-3}$, and $g$ is the acceleration due to gravity in $\mathrm{ms}^{-2}$.

While permeability is an intrinsic quantity the hydraulic conductivity is not, as it depends on the density and viscosity which in turn depend on the temperature, pressure and the nature and concentration of solutes in water. From equation (4.3) it can be seen that for 
a given fluid the permeability and hydraulic conductivity are directly proportional. The success of efforts to find a link between SIP and hydraulic conductivity depends on the accuracy with which the SIP parameters and the hydraulic conductivity are measured. There are two potential laboratory methods for determination of hydraulic conductivity. The first is called the constant head method and the second is the falling head method. The choice of method depends on the hydraulic conductivity of the material. The falling head method is generally applicable for materials with hydraulic conductivities ranging from $10^{-9}$ to $10^{-5} \mathrm{~ms}^{-1}$, while the constant head method is generally applicable to materials with hydraulic conductivity ranging from $10^{-5}$ to $10^{-3} \mathrm{~ms}^{-1}$ (Nielsen, 1991). The hydraulic conductivity of all the samples dealt with during this project is between $10^{-5}$ to $10^{-3} \mathrm{~ms}^{-1}$ hence the constant head method was used throughout.

\subsubsection{The permeameter}

The permeameter consists of a cylindrical column with inlet and outlet for entry and exit of water. The water flows through the cylindrical column from the high pressure region to the low pressure region. The sample holder design enables it to also be used as the cylindrical column for the permeameter (Figures 4.12 and 4.13). The set up to measure hydraulic conductivity consists of a specially designed wooden 'stand' as shown in the Figure 4.12. On top of the stand a large funnel is fixed. This funnel is provided with an overflow. Water is continuously poured into the funnel from a large tank that is kept above the funnel. The funnel and overflow act as the constant pressure head for the permeameter. The sample holder is mounted on the wooden stand with the help of two wooden holders. It is possible to move the sample holder along the wooden stand so that it can be fixed at any point on the wooden stand. This allows a variation of pressure head to be applied to the sample. The hydraulic conductivity is calculated using Darcy's law as follows.

$K=\frac{Q L}{A H}$

Where $K$ is the hydraulic conductivity in $\mathrm{ms}^{-1}, Q$ is the volume of water collected in unit time in $\mathrm{m}^{3} \mathrm{~s}^{-1}, A$ is the cross-sectional area of the sample perpendicular to the flow direction in $\mathrm{m}^{2}$, and $H$ is the hydraulic head difference between the ends of the sample in $\mathrm{m}$. 


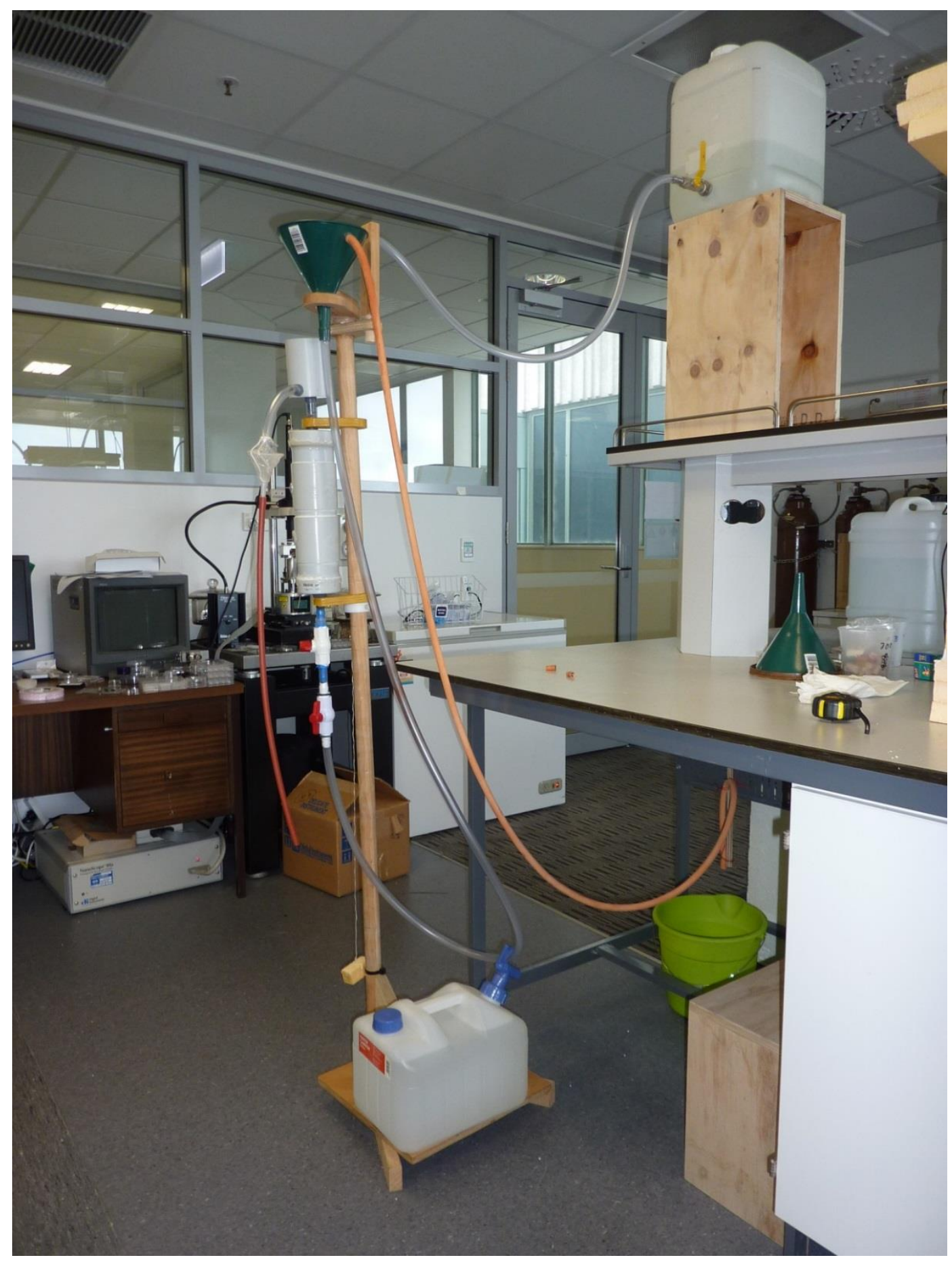

Figure 4.12: The set up for of hydraulic conductivity measurement.

For Darcy's law to be valid, certain conditions have to be met. One of the conditions is that the fluid has to be in laminar flow (Bear, 1972). Hence, very high water flow speeds are not desirable for hydraulic conductivity estimation. To avoid any unwanted effects which may invalidate the use of Darcy's law, the pressure head is varied according to the hydraulic conductivity of the sample. 
A stopwatch is used to measure the time for which water is collected and the mass of the water collected is determined with an electronic scale. Hence

$$
Q=\frac{V}{t}=\frac{m}{\rho t}
$$

where $m$ is the mass of water collected during an interval of $t$ and $\rho$ is the density of water.

The water used for the hydraulic conductivity measurement was distilled and the electrical conductivity was carefully controlled by adding table salt. The water that flows out of the outlet of the permeameter is re-used for maintaining the constant water supply as the capacity of the water tank is only 20 litre.It is not viable o obtain a continuous supply of distilled water. Before re-use the water was filtered using a $50 \mu \mathrm{m}$ filter to remove any sediment which might settle on the current electrode. It was found in some early measurements that some biological mass (most probably algae) did settle on the current electrode and tended to cause a successively reducing value of hydraulic conductivity. The electrical conductivity also was checked and corrected before the water was re-used.

\subsection{The sample holder}

When a sample holder is filled with an unconsolidated material like sand, the sand forms a certain structure inside the sample holder. This structure controls the quantities like porosity, formation factor, pore size, hydraulic conductivity; and therefore also the ColeCole relaxation time constant derived from an SIP spectrum and used to predict the hydraulic conductivity. This structure is influenced by the method adopted for filling the sample holder, and there is a degree of uncertainty with which the structure can be replicated for successive tests. Any transfer from one sample holder to another, or even movement of the sample in the sample holder itself, can change the structure of the porous medium. Therefore hydraulic conductivity, formation factor and SIP measurements were carried one after the other without changing sample holders or disturbing the sample. The materials used in constructing the sample holder were easily available materials like PVC pipe and fitting parts that are commonly used in plumbing; such as a straight couplers and end caps. No metal parts were used except for the electrodes as they can influence the SIP spectra. 


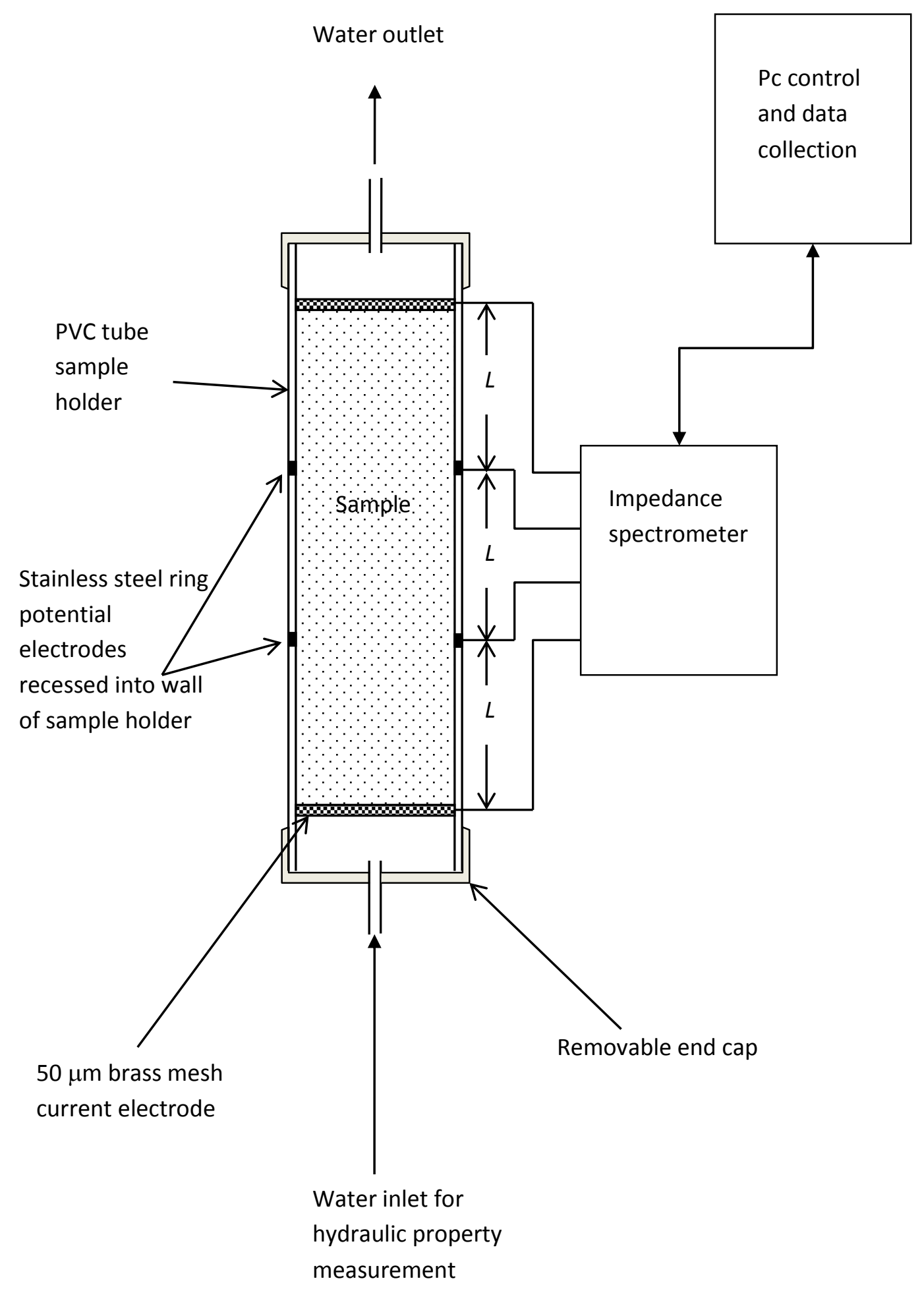

Figure 4.13: Schematic of the sample holder and measurement system used for SIP measurements.

\subsubsection{The current and potential electrodes}

The low polarization of the samples demands the use of a four-electrode method for the measurement of impedance (Kemna et al., 2012). The phase component of a typical SIP response is of the order, at most, a few tens of milli-radians; hence, it is important to 
minimize all other factors than can affect the impedance. In the case of laboratory measurements, the factor that is most likely to affect the impedance measurement is electrode polarization; discussed in section 3.2.1. One solution to avoid electrode polarization is the use of non-polarizing electrodes. Various types of non-polarizing electrodes are used for SIP measurements including water electrodes. Such nonpolarizing electrodes have the disadvantage that they make contact with only one point in the sample. For impedance spectrometers with high input impedances, non-polarizing electrodes are not a strict requirement (Kemna et al., 2012). Stainless steel is a commonly used electrode material both in the laboratory and field measurements. Other metals like copper, and alloys like brass and bronze, can also be used. The metal electrode can be a ring electrode or a point electrode. One solution to reduce electrode polarization in potential electrodes is to remove the metal potential electrode from the current path by placing it in contact with the sample at the edge of the current flow (Vineagar and Waxman, 1984). This can be achieved by using ring electrodes as illustrated in Figure 4.13.

\subsubsection{Construction of the sample holder}

During this project a number of sample holders were tried and one with the following dimensions was finally selected. However, some of the earlier measurements were made with sample holders with slightly different dimensions. The sample holder is made up of a PVC pipe of radius $0.038 \mathrm{~m}$ and a total length of $0.3 \mathrm{~m}$. The size of the sample holder was finalized by considering the fact that a larger sample holder would require a larger quantity of sample and also may lead to inhomogeneous packing (Kemna et al., 2012). Figure 4.13 shows a schematic of the sample holder. For hydraulic conductivity measurements the sample holder is mounted on the stand in such a way that after hydraulic conductivity and formation factor measurements it can be removed from the stand without disturbing the sand inside the sample holder. The design of the stand and the sample holder is such that there is no scope of losing water from the sample holder at any point during the measurements.

The main parts of the sample holder are: (1) the three PVC pipe pieces which are of length $100 \mathrm{~mm}$ and inner radius $38 \mathrm{~mm}$ each as shown in Figure 4.14; (2) two straight connectors with the potential electrodes fitted in them; and (3) two end caps with the current electrodes inserted in them. The last two components are assembled separately 
and then all three parts are put together to complete the construction of the sample holder.

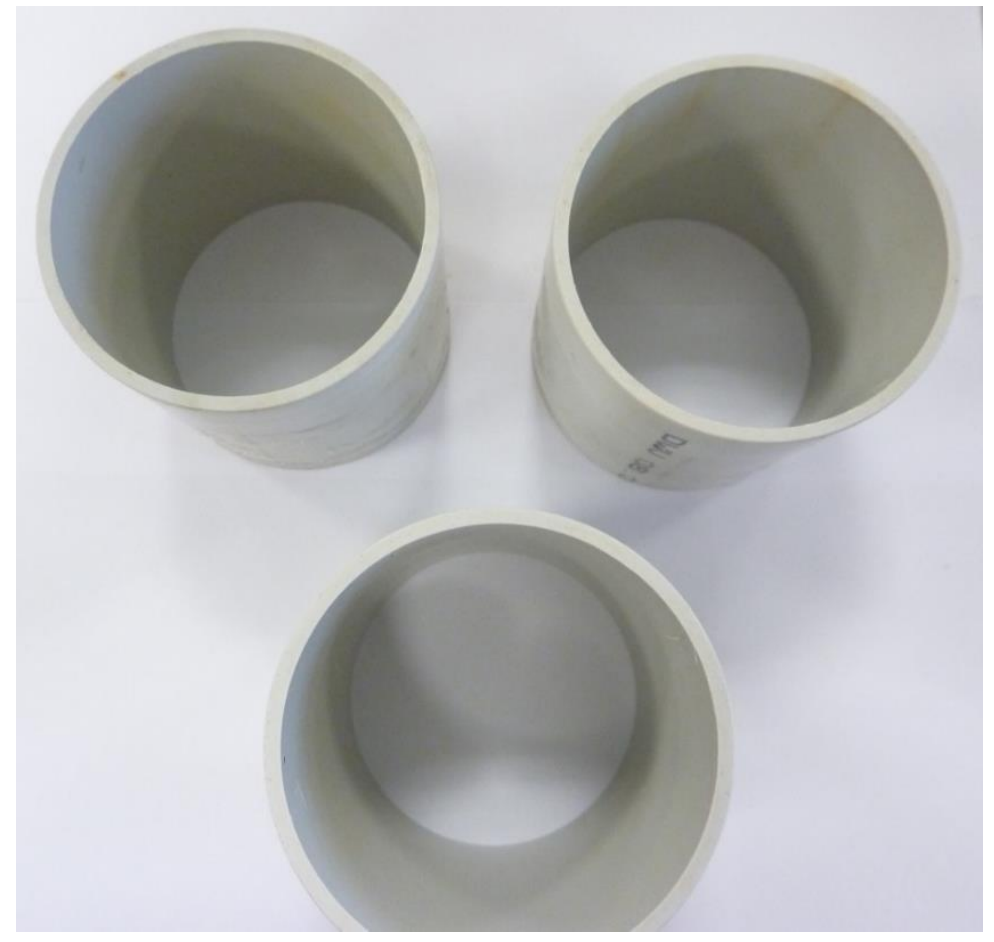

Figure 4.14: PVC pipes used in construction of the sample holder.

Figure 4.15(a) shows the rings used as the potential electrodes. They are made of 316 grade stainless steel rod of diameter $1 \mathrm{~mm}$. The diameter of the ring is such that it fits tightly inside the straight connector. The ring is provided with an extension which passes through a hole in the straight connector. This is used to make electrical connections to the potential electrode. Figure 15(b) shows the potential electrodes fitted inside the straight connector.

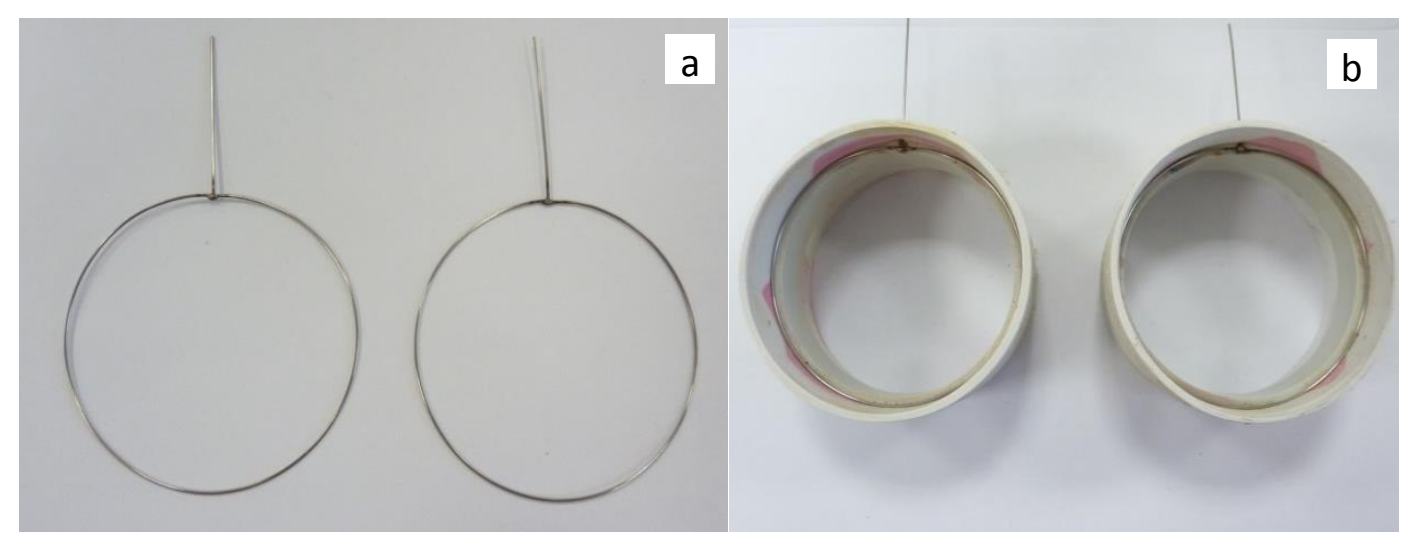

Figure 4.15: (a) Stainless steel rings used as potential electrodes; (b) after insertion into PVC pipes. 


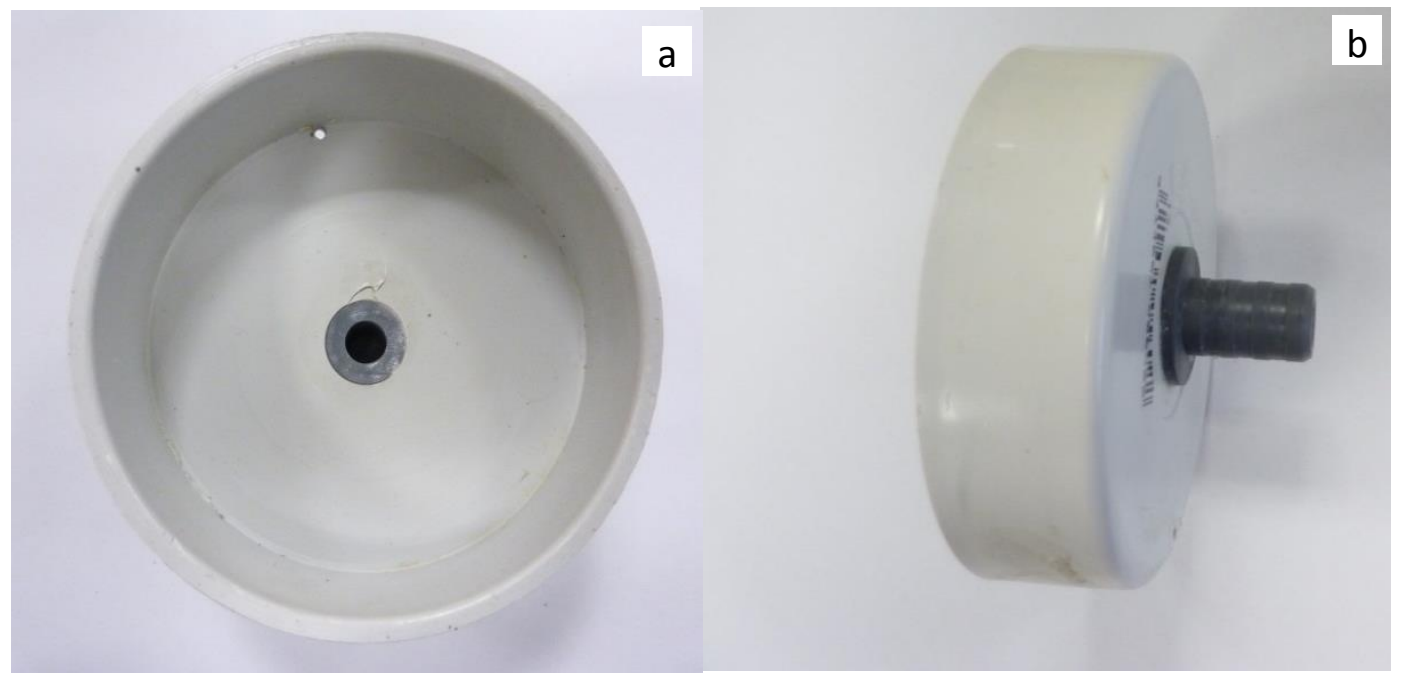

Figure 4.16: End (a) and side (b) views of the end caps into which the current electrodes are inserted.

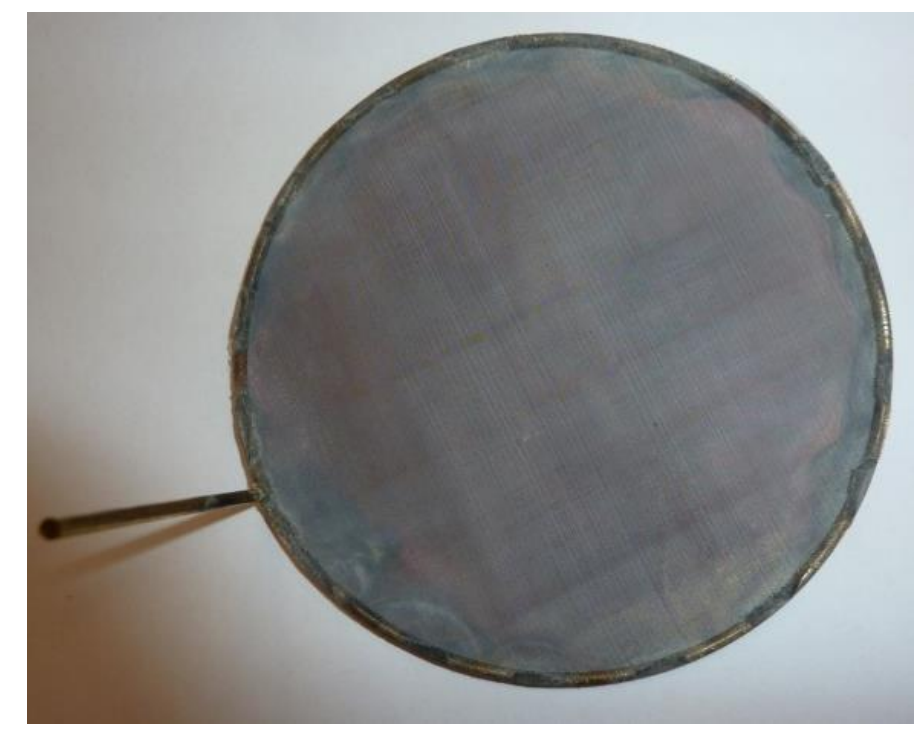

Figure 4.17: A mesh current electrode.

Figures 4.16(a) and (b) respectively show the front and the side view of the end cap prepared to fix the current electrode. A hose tail is fixed at the centre of the end cap for connecting water inlet/outlet pipes. The end cap has a small hole through which a brass rod soldered onto the current electrode can pass through for making electrical connections.

Figure 4.17 shows the current electrode. It is made by soldering 50 micron brass mesh onto a ring made of brass rod of diameter $1.6 \mathrm{~mm}$. A short brass rod is soldered onto the brass ring perpendicular to the plane of the brass mesh for making electrical connections. 
The current electrode is then inserted into the end cap. Figures 4.18(a) and (b) respectively show the front and side view of the end cap with the current electrode in place.

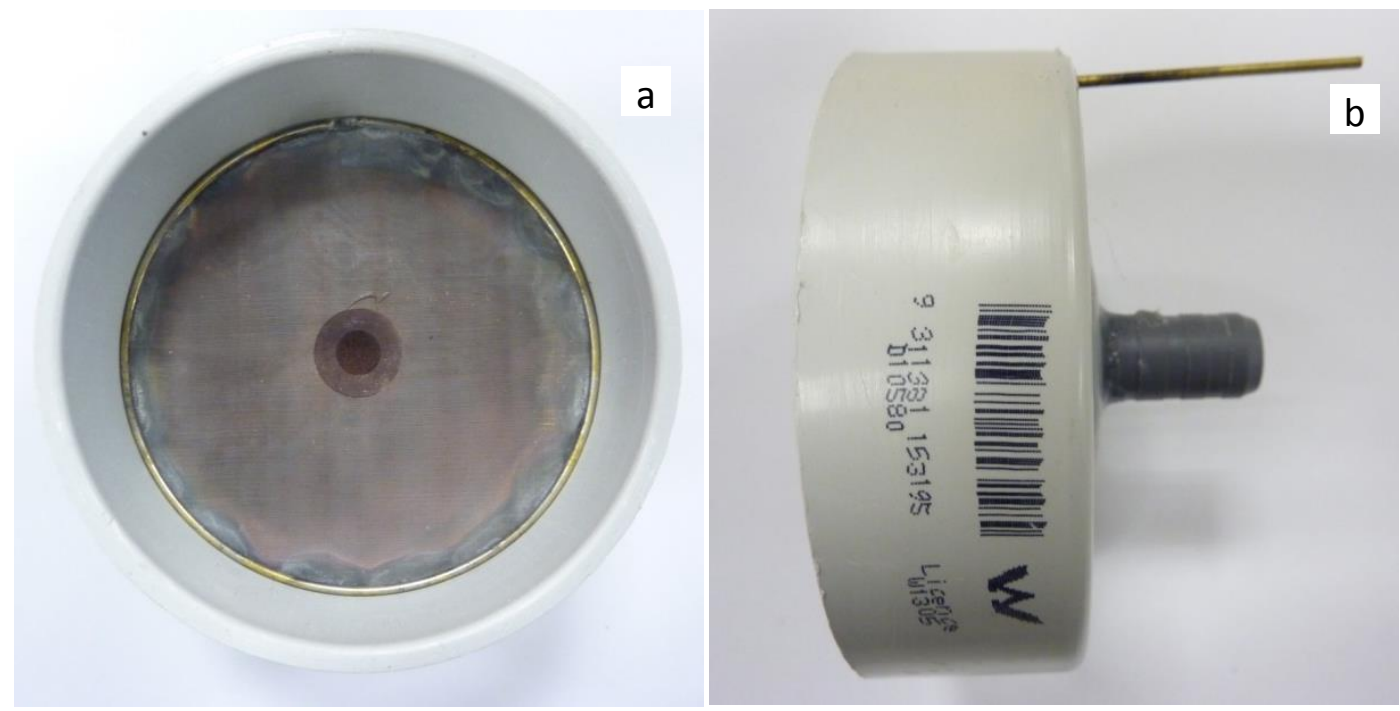

Figure 4.18: End (a) and side (b) views of the end caps after insertion of the current electrode.

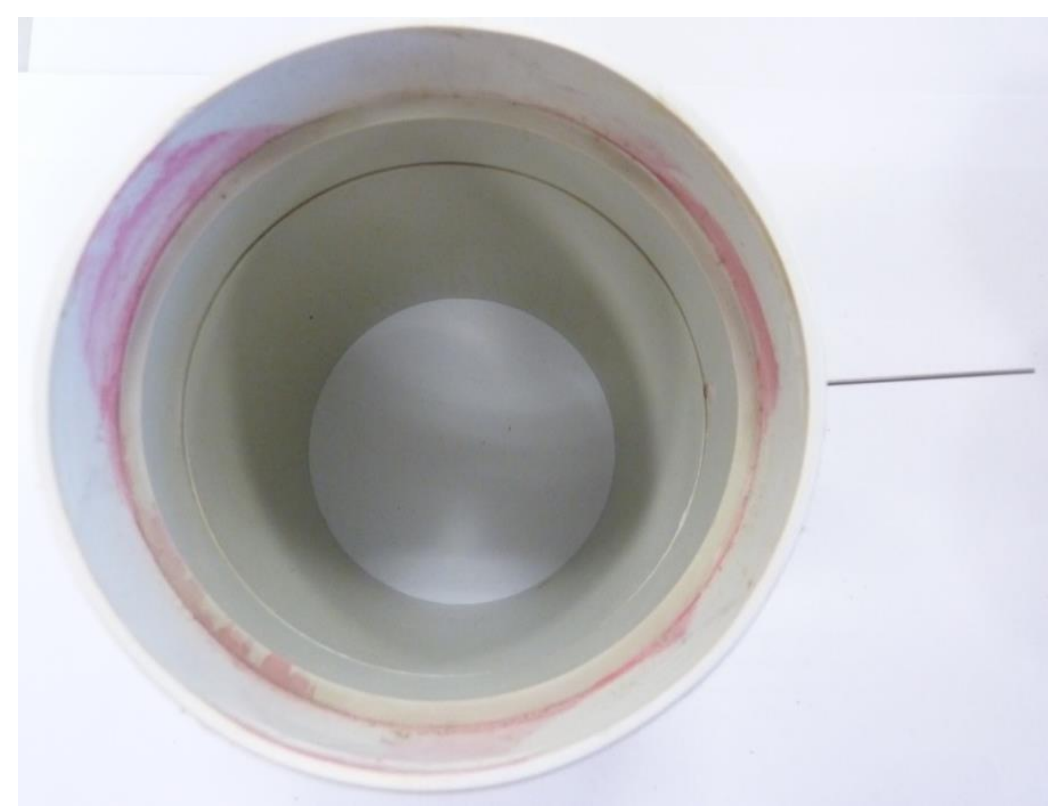

Figure 4.19: PVC pipe and straight connector joined together so that the ring potential electrode sits in the groove between them. 
At this stage the three components are ready to be joined together. First the two straight connectors are fitted on the two ends of the one of the three PVC pipes. Note that when the straight connector is connected to the PVC pipe the ring electrode sits inside the groove formed between the PVC pipe and the straight connector as shown in Figure 4.19. As the potential electrodes are situated inside the groves, they do not enter the path of current flowing in the sample. This reduces any electrode polarization effects.

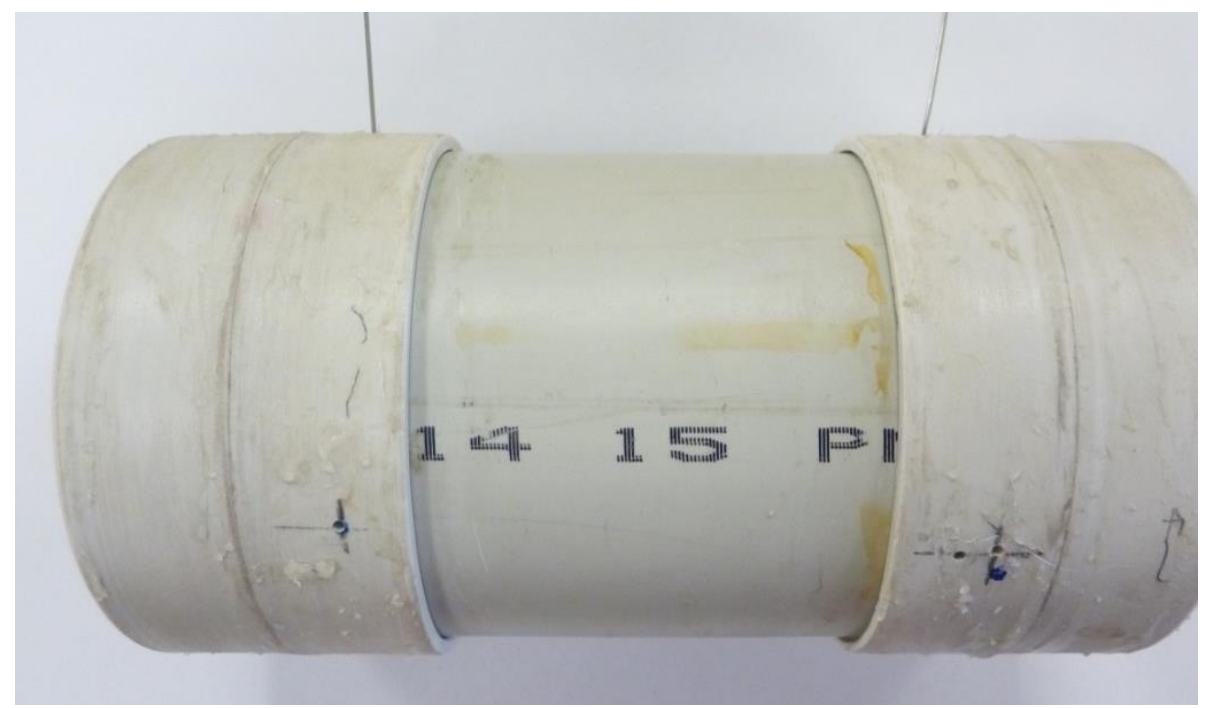

Figure 4.20: Sample holder with potential electrodes in place.

Figure 4.20 shows the sample holder after both straight connectors, with potential electrodes, inserted in the PVC pipe. The remaining two pipes are connected as shown in figure 4.21, and in the last stage the sample holder is closed at both ends with the end caps into which the current electrodes are attached. The end cap at one end of the sample holder is sealed with a sealant while the other end can be conveniently opened

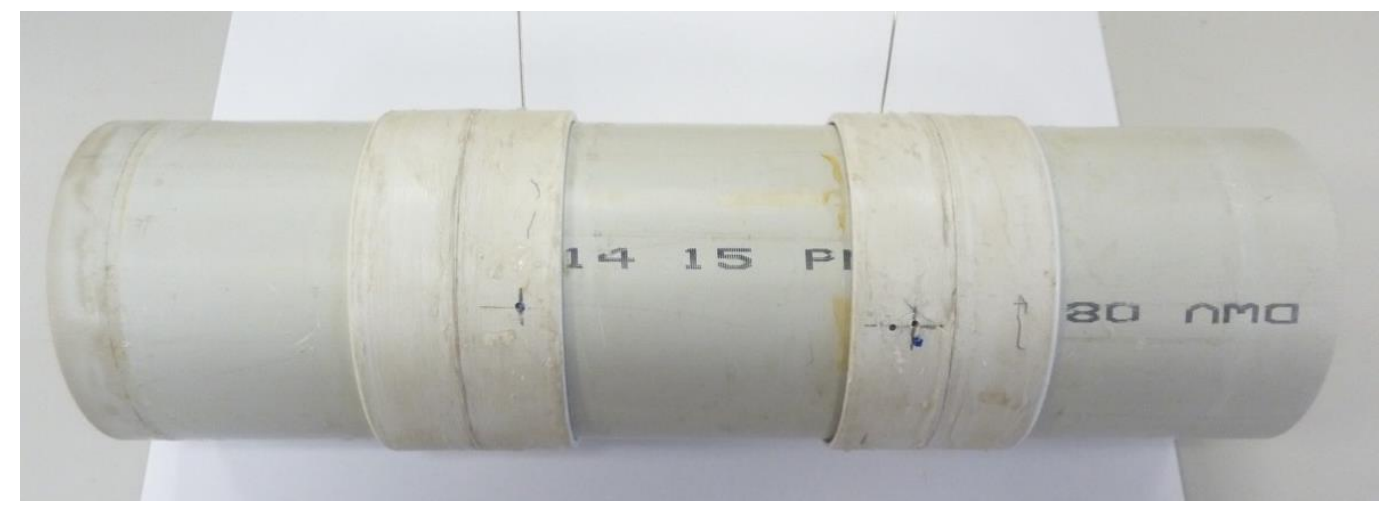

Figure 4.21: The connected straight pieces with the potential electrodes in place. 
and closed. After filling with the sample the end cap is sealed using hot melt glue. The completed sample holder is shown in Figure 4.22.

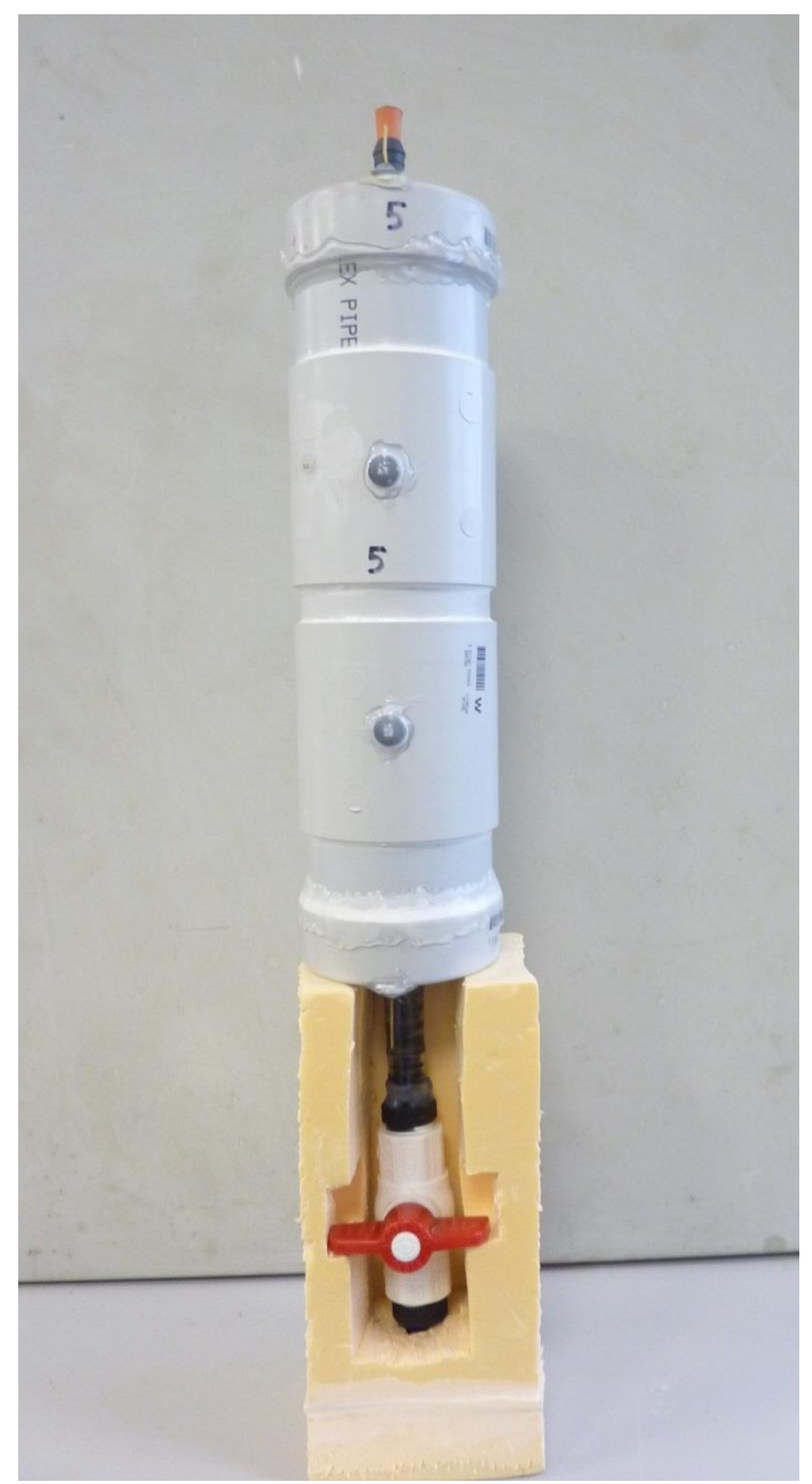

Figure 4.22: The completed sample holder. 


\subsection{Sample preparation and packing}

Samples were collected from five different locations in the lower North Island. Most measurements were made on sand collected from the beds of the Hutt and Rangitikei Rivers. Other sources of samples were Waitarere Beach, and the Koputaroa sand dunes. Figure 4.23 shows the locations of sample collection.

The sample collected is first cleaned of biomass. This is to prevent blocking of the current electrode mesh. The sample is then repeatedly washed in tap water. Exceptional care is taken while washing to avoid losing the finer grains. Beach sand requires a more thorough washing compared to river sand as it contains salt. The washed sand is dried in an oven at $110^{\circ} \mathrm{C}$ for two hours. Samples used in the study of variation of hydraulic conductivity with grain size are prepared by sieving the natural sample into different fractions. The sizes of the sieve meshes used differ successively by $0.25 \varphi$ as defined by the Krumbein Phi $(\varphi)$ scale (Krumbein and Aberdeen, 1937). The Krumbein Phi scale is a logarithmic scale computed by the expression

$\varphi=\log _{2}\left(\frac{D}{D_{0}}\right)$

where $\varphi$ is the Krumbein Phi, $D$ is the diameter of the particle, and $D_{0}$ is a reference diameter equal to $1 \mathrm{~mm}$. Table 4.3 gives the size of the sieves used in Krumbein Phi scale and the range of diameters of sand collected on each of the meshes.

Sample packing is one of the biggest challenges in laboratory SIP measurements. There is a suggestion that the SIP effect is governed by the pore/pore throat size rather than by the grain size (Revil et al., 2012). Obviously the pore/pore throat size will depend on the method of sample packing; for example, if the sand was packed wet or dry, or it was compacted by some means etc. Hence the sample preparation method can have a significant impact on the resulting SIP spectrum. Figure 4.24 from (Kemna et al., 2012) demonstrates the effect on SIP spectra resulting from changes in packing and saturation.

Even though sample preparation is critical, it appears to have been largely ignored in the literature. Reproducible measurements are necessary to compare results, especially of different working groups and experimenters, and to find universally valid statements for unconsolidated sediments. 


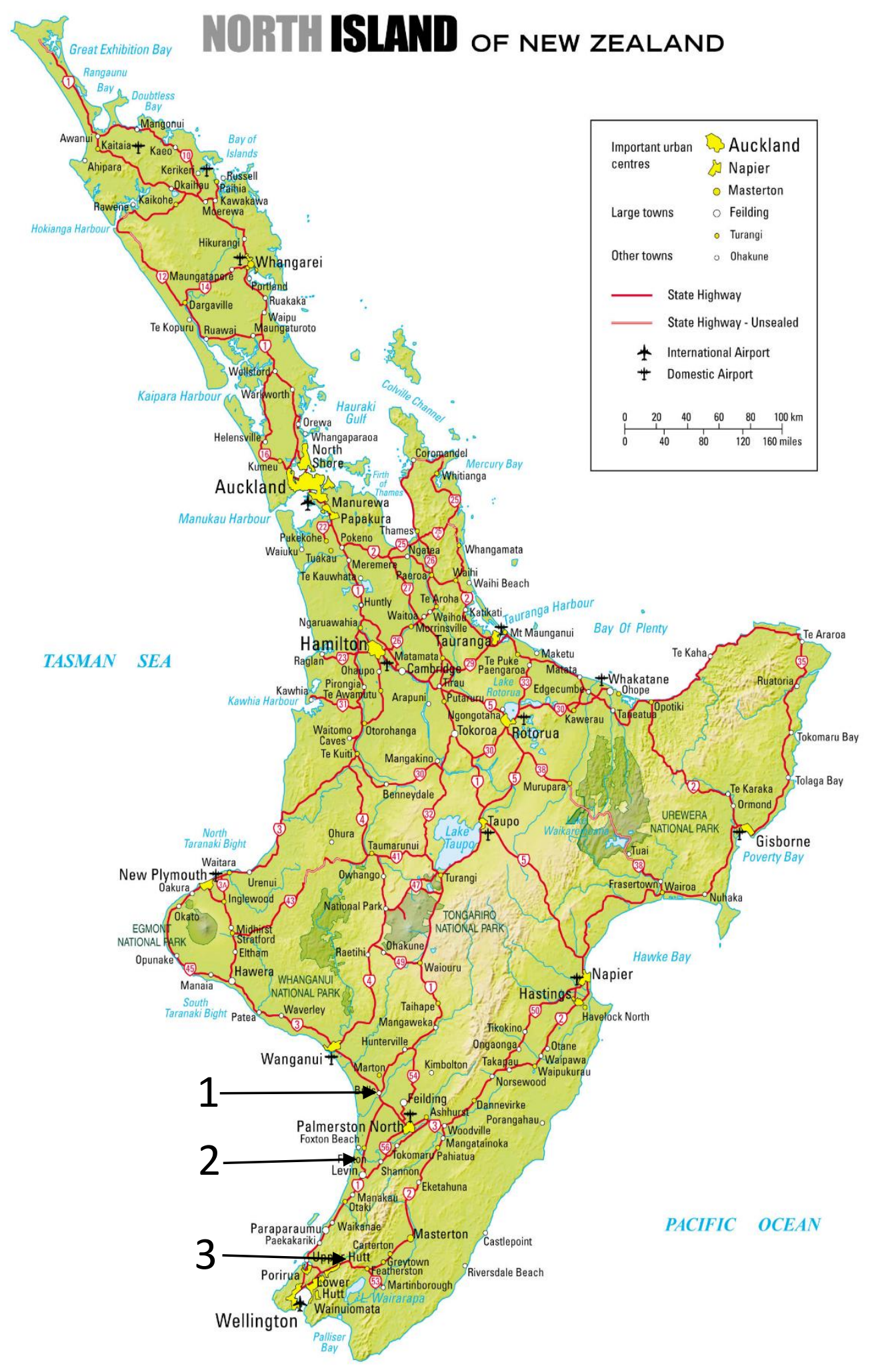

Figure 4.23: Locations from where samples were collected. (1) Rangitikei River. (2) Waiterere beach. (3) Hutt River (http://newzealandatitsbest.com/node/55). 
Although this is an essential issue of laboratory IP-measurements (Kemna et al., 2012) ; (Slater and Glaser, 2003), studies concerning the influence of the preparation method are sparse (Bairlein et al., 2014). The reproducibility of SIP measurements of sand posed a challenge for the sample preparation in this project also.

\begin{tabular}{|c|c|c|c|}
\hline $\begin{array}{c}\text { Mesh size in } \\
\text { Krumbein } \varphi \\
\text { Scale }\end{array}$ & $\begin{array}{c}\text { Mesh size } \\
\text { in } \mu \mathrm{m}\end{array}$ & $\begin{array}{c}\text { Range of grain } \\
\text { diameter in } \mu \mathrm{m}\end{array}$ & $\begin{array}{c}\text { Median grain } \\
\text { diameter in } \mu \mathrm{m}\end{array}$ \\
\hline 0 & 1000 & $1189.21-1000.00$ & 1094.61 \\
\hline 0.25 & 840.9 & $1000.00-840.90$ & 920.45 \\
\hline 0.5 & 707.11 & $840.90-707.11$ & 774 \\
\hline 0.75 & 594.6 & $707.11-594.60$ & 650.86 \\
\hline 1 & 500 & $594.60-500.00$ & 547.3 \\
\hline 1.25 & 420.45 & $500.00-420.45$ & 460.22 \\
\hline 1.5 & 353.55 & $420.45-353.55$ & 387 \\
\hline 1.75 & 297.3 & $353.55-297.30$ & 325.43 \\
\hline 2 & 250 & $297.30-250.00$ & 273.65 \\
\hline 2.25 & 210.22 & $250.00-210.22$ & 230.11 \\
\hline 2.5 & 176.78 & $210.22-176.78$ & 193.5 \\
\hline 2.75 & 148.65 & $176.78-148.65$ & 162.71 \\
\hline 3 & 125 & $148.65-125.00$ & 136.83 \\
\hline
\end{tabular}

Table 4.3: Mesh sizes and corresponding ranges of sample grain diameters.

Initially a sample was prepared by filling water saturated sand into the sample holder. But this method has a drawback as it is difficult to control the porosity of the sample prepared. A decrease in porosity causes an increase in formation factor and a decrease in the hydraulic conductivity. As a result, the relaxation time constant also tends to decrease. This variation in porosity poses a problem when it comes to the investigation of variation of hydraulic conductivity with grain size. The variations in the hydraulic conductivity caused by variations in porosities of the respective samples itself is found to be greater than the expected variation from the grain size. Therefore the experiment demands a sample preparation method that is capable of controlling the porosity much more precisely.

The second method of sample preparation involves filling the sample holder with dry sand. This has the advantage that the porosity can be precisely controlled by varying the 
amount of dry sand filled into the sample holder. After a number of trials the following method was adopted for filling the sample holder. The sieved sand is dried in the oven at $100^{\circ} \mathrm{C}$ for 2 hours to completely remove moisture. It takes about $2.1-2.2 \mathrm{~kg}$ of sample to fill the sample holder to produce a sample that has a porosity of $40 \pm 1 \%$. About $50 \mathrm{~g}$ of dry sand is poured into the sample holder and, after closing the sample holder, it is shaken well. While shaking the sample holder is overturned at regular intervals. This is to avoid vertical sorting effects i.e. the bigger grains moving towards the surface and smaller grains accumulating at the bottom (Revil et al., 2012a). This is repeated 4 times i.e. after each filling of $50 \mathrm{~g}$ of sand the sample holder is shaken about 40 times, turning it upside down after 5 shakes. The shaking involves vertically lifting and lowering the sample at a high velocity.
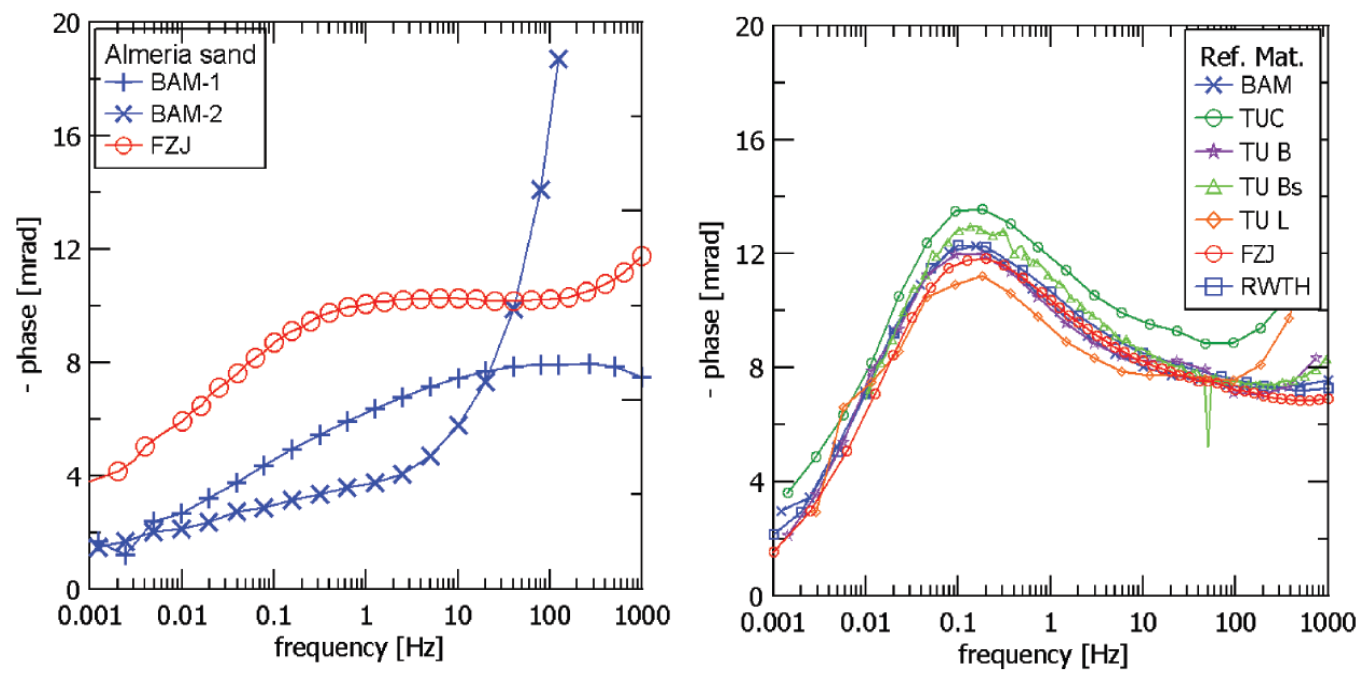

Figure 4.24: (Left) SIP spectra of a sand sample (from an aquifer in Almeria, Spain) acquired intentionally with different sample preparation methods (FZJ: sample highly compacted, BAM-1: sample loosely compacted, BAM-2: sample loosely compacted and use of non-optimized sample holder). (Right) Comparative measurements on BAM reference material over 3 years at six German institutions. BAM: Federal Institute for Materials Research and Testing, Berlin; TU C: Clausthal Technical University; TU B: Berlin Technical University; TU Bs: Braunschweig Technical University; TU L: Leipzig University; FZJ: Jülich Research Centre; RWTH: Aachen Technical University (Kemna et al., 2012).

After the sample holder is almost completely filled, it is shaken a few more times to make sure that the sand is tightly packed. To obtain an accurate value of hydraulic 
conductivity a number of repeat measurements are required. If the sample holder is not tightly packed there will be compaction while water flows through the sample. If the packing of the sand is not tight enough there can therefore be a continuous decrease in the measured hydraulic conductivity from the increasing compaction or settling of the sample during the flow of water. This compaction will also affect the value of electrical formation factor. The above mentioned procedure ensures that variations in porosity, formation factor, and hydraulic conductivity do not happen when water flows through the sample. It is found that the maximum porosity value that does not lead to a variation in packing during the measurement is around $40 \%$. This porosity is the minimum value that can be achieved with reasonable effort in the lab. This is further justified when we consider the fact that in the field

\subsection{Measurement of porosity}

The porosity is a measure of void/empty space in a material. It is defined as the ratio of void space to the total volume of the material. Hence its value varies from 0 to 1 .

$\Phi=\frac{V_{V}}{V_{T}}$

Here $\Phi$ is the porosity, $V_{V}$ is the void space, and $V_{T}$ is the total volume of the material.

The pore volume $V_{V}$ of the filled sample holder is equal to the volume of water that is used to saturate the sample and the total volume $V_{T}$ is equal to volume of the sample holder itself. The volume of water required to fully saturate the sample can be calculated by weighing the sample holder before and after saturation. If $W_{d}$ and $W_{s}$ are the masses of the sample holder before and after saturation, then the porosity of the sample is given by

$\Phi=\frac{W_{s}-W_{d}}{\rho \pi r^{2} l}$

where $\rho$ is the density of the fluid, $r$ is the inner radius of the sample holder, and $l$ is the length of the sample holder as shown in Figure 4.13. The error in measurement of porosity is around $6 \%$. 


\subsection{Determination of the electrical formation factor}

The electrical resistivity of a saturated rock or sediment is a function of the porosity, the electrical resistivity of the saturating fluid, the resistivity of the solid rock or soil, the surface conductance of the rock or soil, and the tortuosity of the fluid and electrical path [Huntley, 1986]. The ratio of total measured resistivity of the porous material to the resistivity of the fluid that fills the pore space of the porous medium is called the electrical formation factor, and it is an important quantity in geophysics. At very low resistivities of the pore fluid, the entire current is conducted through the pore fluid. In such cases the electrical formation factor does not vary with the electrical resistivity of the pore fluid and is called the intrinsic formation factor. But at high resistivity values of pore fluid conductivity, the contribution from surface conductivity becomes significant. In such cases a parallel resistor model is used to accommodate the electrical conductance other than that through the fluid (Huntley, 1986). This can be expressed as follows

$\frac{1}{R_{t}}=\frac{1}{R_{w}}+\frac{1}{R_{g}}+\frac{1}{R_{S}}$

where $R_{t}$ is the bulk measured resistance of the sample, $R_{w}$ is the resistance of the path through the pore fluid, $R_{g}$ is the resistance of the grains or rock and $R_{S}$ is the resistance along the surface of grains or rock due to surface conductance effects referred to as matrix conduction. This matrix conduction has significance for the determination of electrical formation factor. For a medium with significant matrix conduction the formation factor measured at higher fluid resistivities will be less than the intrinsic formation factor and it is called the 'apparent' formation factor $\left(F_{a}\right)$. Figure 4.25 shows the variation of apparent formation factor with resistivity of the pore fluid. The magnitude of the electrical resistance $\left(R_{g}\right)$ of the rock or grains is large compared to other contributions and hence it can be neglected, then the equation (4.9) becomes

$\frac{1}{R_{t}}=\frac{1}{R_{w}}+\frac{1}{R_{S}}$

Expressing the resistances in terms of the corresponding electrical resistivities the above equation can be written as

$\frac{1}{\rho_{t}}=\frac{1}{\rho_{w} F}+\frac{1}{\rho_{s}}$ 
where $\rho_{t}$ is the measured bulk resistivity, and the resistivity of the path through the pore fluid has been written in terms of the actual resistivity $\left(\rho_{w}\right)$ of the pore fluid and $F$ the intrinsic electrical formation factor, $\rho_{S}$ is the matrix resistivity. Expressing this in terms of respective electrical conductivties equation (4.11) becomes

$\sigma_{t}=\frac{\sigma_{w}}{F}+\sigma_{s}$

the above equation can also be written as

$\sigma_{w}=\sigma_{t} F-\sigma_{s} F$

and

$F_{a}=\frac{F \sigma_{w}}{\sigma_{s} F+\sigma_{w}}$

From equation (4.13) it can be seen that the gradient of a plot of $\sigma_{w}$ vs $\sigma_{t}$ will give the true/intrinsic formation factor $F$. An equation similar to (4.14) was used by Weller et al. (2013) to describe the dependence of apparent formation facton on pore fluid conductivity.

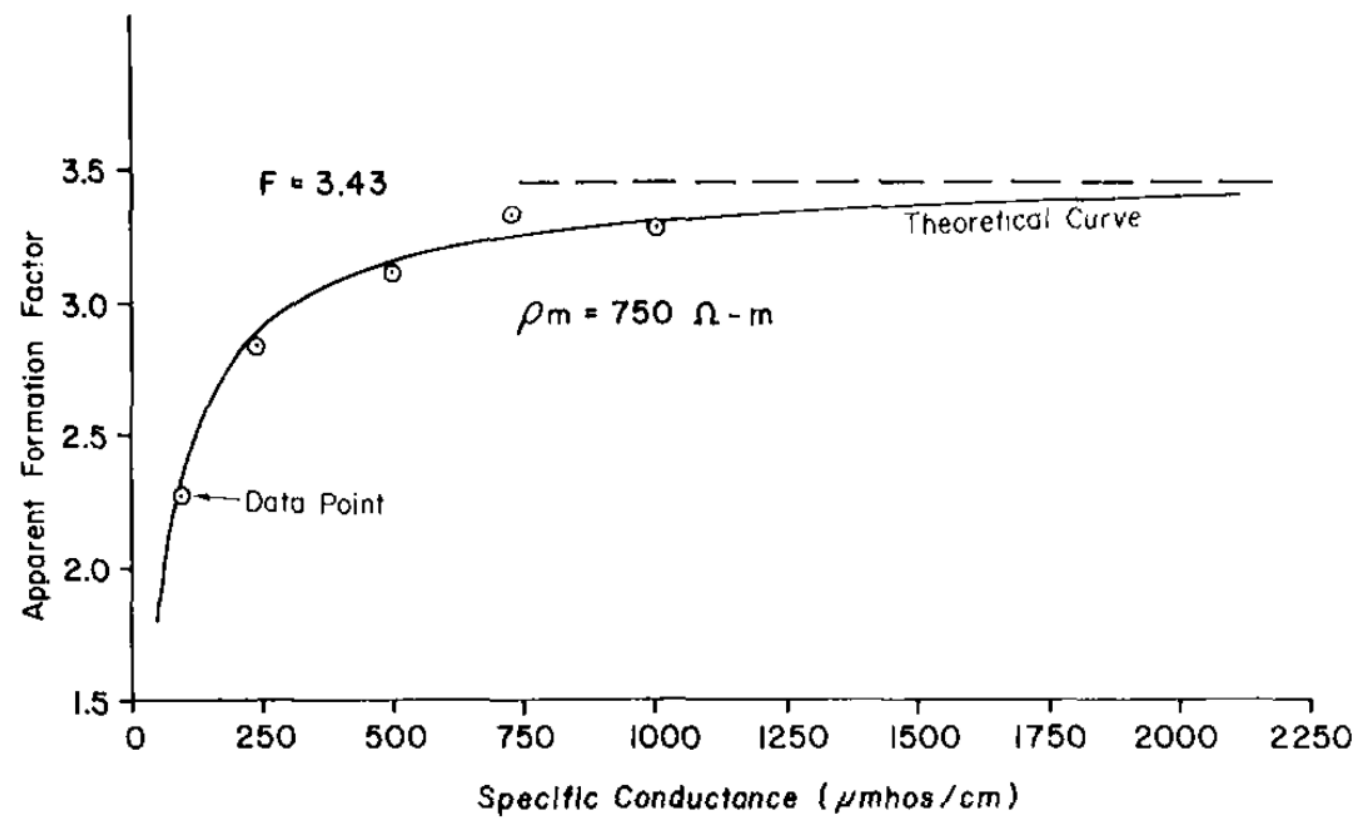

Figure 4.25: Variation of apparent formation factor with fluid specific conductance for well-sorted, clay-free, medium grained sand (Huntley, 1986). 


\subsubsection{Experimental method to determine electrical formation factor}

From equation (4.13) it can be seen that the gradient of the plot of pore fluid conductivity against the measured bulk conductivity gives the intrinsic formation factor. Such a plot can used to determine the electrical formation factor as shown in Figure 4.26. In order to determine the electrical formation of the samples studied in this project the electrical conductivity of the sample was measured at three different electrical conductivity of the pore fluid. Then the intrinsic formation factor was determined from the values of pore fluid conductivity and bulk conductivity of the sample. This was done in Microsoft Excel using the LINEST() function. The formation factor of unconsolidated sediments varies according to the porosity/compaction. In the case of clay free sand samples with porosity around $40 \%$ the formation factor is about 3.8 .

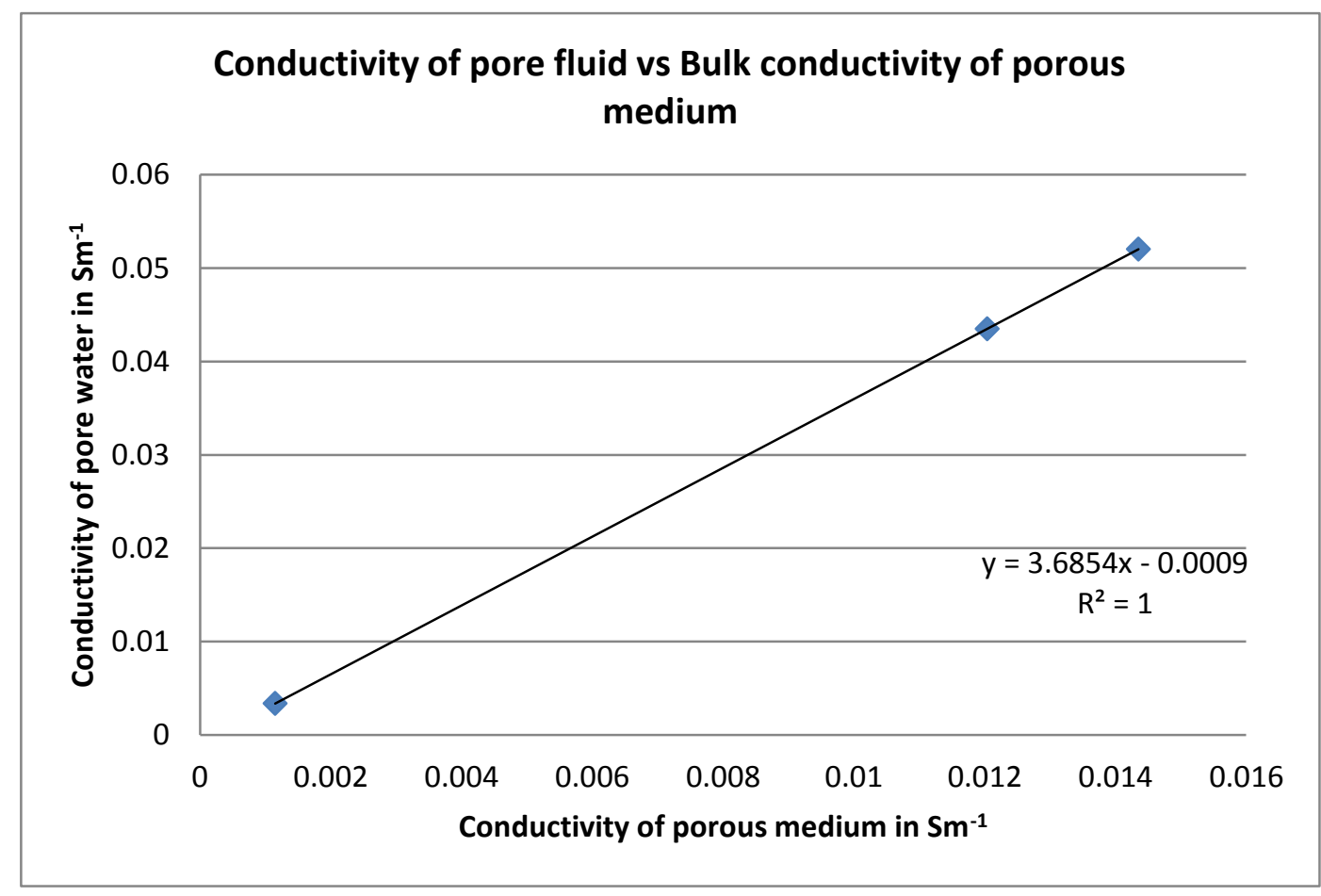

Figure 4.26: Determination of the intrinsic formation factor.

\subsubsection{A method for estimating intrinsic formation factor from a single apparent formation factor}

For some of the initial samples the electrical resistivity was measured only at a single electrical conductivity of the pore water that too at a relatively low electrical conductivity. Hence as discussed earlier, the formation factor calculated from a single 
electrical conductivity of pore fluid will be an underestimate of the intrinsic formation factor, and hence it requires a correction. A method for estimating intrinsic formation factor is given by (Weller et al., 2013). According to them the apparent formation factor is

$F_{a}=\frac{F \sigma_{w}}{a+\sigma_{w}}$

where $F_{a}$ is the apparent formation factor, $F$ is the intrinsic formation factor, $\sigma_{w}$ is the electrical conductivity of pore water, and $a$ is a free parameter that correspond to the product of $F \sigma_{\text {surf }}^{\prime}$. Note that this equation is equivalent to (4.14) above with $a=\sigma_{s} F$. If the value of $a$ is known, and the apparent formation factor is measured at a known single fluid conductivity, the intrinsic formation factor can be calculated.

\subsection{Measurement of electrical conductivity of pore fluid.}

The electrical conductivity of the pore fluid was measured using the YSI Model 3100 conductivity meter. The pore fluid conductivity is measured by dipping the conductivity cell into the fluid. The YSI Model 3100 is a microprocessor based instrument designed to perform laboratory measurements of conductivity, salinity and temperature. The Model 3100's microprocessor allows the system to be calibrated with the press of few

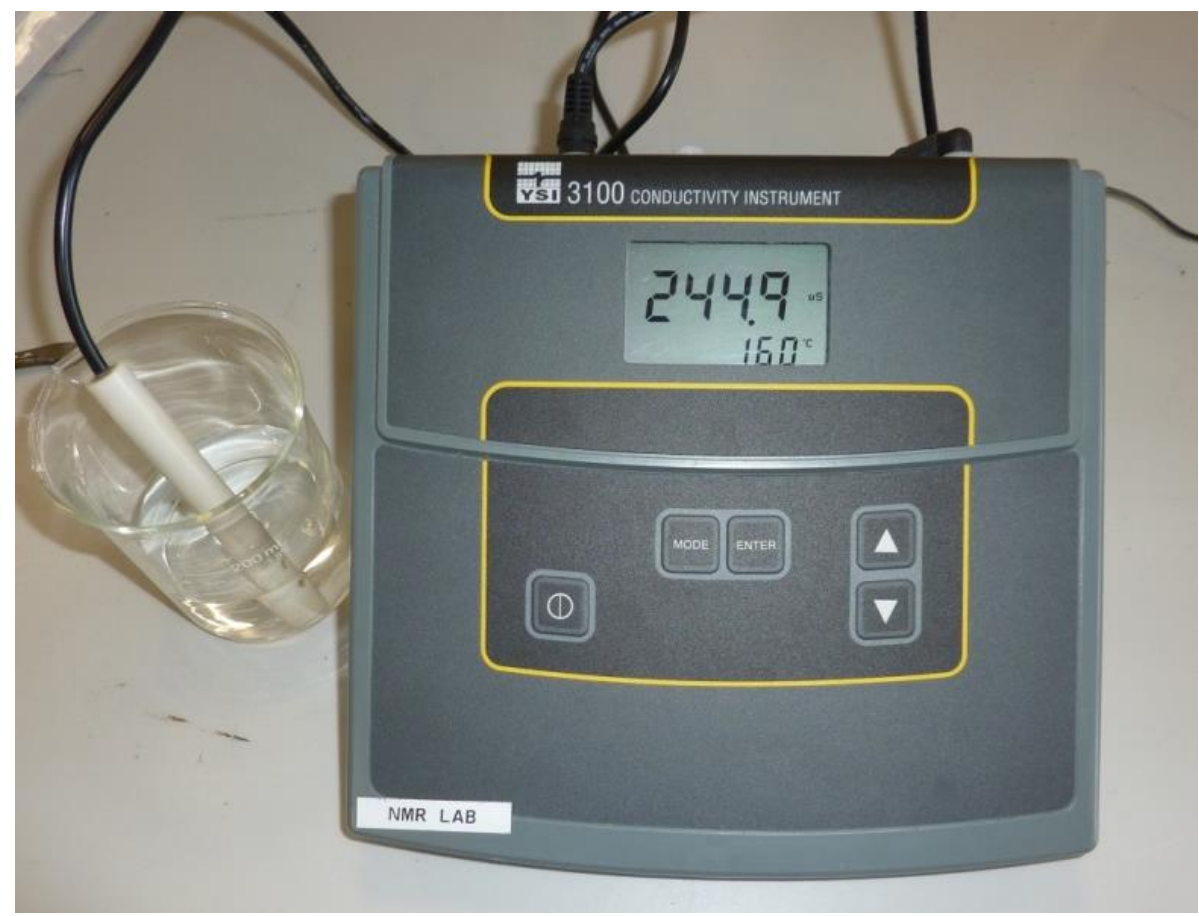

Figure 4.27: The YSI Model 3100 conductivity meter. 
keys. Additionally, the microprocessor performs a self-diagnostic routine each time the instrument is turned on. The self-diagnostic routine provides useful information about the cell constant, the function of the instrument circuitry, and the quality of the readings. The system simultaneously displays the temperature (in ${ }^{\circ} \mathrm{C}$ ) along with one of the following parameters: conductivity (in $\mu \mathrm{Scm}^{-1}$ or $\mathrm{mScm}^{-1}$ ), temperature compensated conductivity (in $\mu \mathrm{Scm}^{-1}$ or $\mathrm{mScm}^{-1}$ ), or salinity (in parts per thousand, [ppt]). The user can easily switch back and forth between these modes using the [MODE] key. Figure 4.27 shows a photograph of the conductivity meter.

\subsection{Conclusion}

This chapter details the necessary instrumentation and procedure for laboratory SIP and hydraulic conductivity measurements. The instrument used is a custom made impedance spectrometer which is able to make accurate phase measurements of 1 milli radian. The sample holder design permits both SIP and hydraulic conductivity measurements to be made without disturbing or moving the sample which is the most important requirement for a study like this. The procedure developed for sample preparation ensures uniformity of samples and hence also ensures that the difference in SIP signal is only due to the variation in grain size and not due to packing effects. The high quality instrumentation, experimental set-up, and the procedure was able to also give accurate measurements of formation factor and porosity. This chapter also explains a how the intrinsic formation factor can be estimated from the apparent formation factor. 


\section{Dependence of SIP on Pore Fluid Conductivity}

\subsection{Introduction}

This chapter reports on the variation of SIP spectra with the conductivity of the pore fluid, and is based on Joseph et al., (2015).

As discussed in Chapter 3.4 the measured bulk conductivity of an unconsolidated material may be expressed in terms of a parallel combination of ionic conductivity through the pore fluid $\left(\sigma_{\text {ionic }}\right)$ and a complex surface conductivity $\left(\sigma_{\text {surf }}\right)$ (Keller and Frischknecht, 1966)

$$
\sigma^{*}=\sigma_{\text {ionic }}+\sigma_{\text {surf }}
$$

In a saturated material, the ionic conductivity may be related directly to the conductivity of the pore fluid $\left(\sigma_{w}\right)$. As discussed in Chapter 4.9, for high pore fluid conductivity, this relationship is through the formation factor $(F)$ (Archie, 1942)

$$
\frac{\sigma_{w}}{\sigma_{\text {ionic }}}=F=\Phi^{-m}
$$

where $\Phi$ is the porosity of the material and $m$ is referred to as the cementation exponent. Combining equations (5.1) and (5.2) leads to

$$
\sigma^{*}=\frac{1}{F} \sigma_{W}+\sigma_{\text {surf }}^{\prime}+\sigma_{\text {surf }}^{\prime \prime}
$$

in which $\sigma_{\text {surf }}^{\prime}$ and $\sigma_{\text {surf }}^{\prime \prime}$ are the in-phase and quadrature-phase parts of the surface conductivity. As outlined in Chapter $3, \sigma_{\text {surf }}^{\prime}$ represents conduction along the surface of grains and pore spaces which is in phase with an applied electric field while $\sigma_{\text {surf }}^{\prime \prime}$ is the result of diffusive polarization effects on the surface. The surface conductivity is frequency dependent and when the bulk conductivity of a saturated material is measured as a function of frequency. Not only does the magnitude of the conductivity vary with frequency but there is also a phase dependence on frequency. Typical phase values are of the order of not more than a few tens of milliradians, reflecting the fact that $\sigma_{\text {ionic }}$ is much greater than either $\sigma_{\text {surf }}^{\prime}$ or $\sigma_{\text {surf }}^{\prime \prime}$.

If useful correlations between SIP and hydraulic parameters are to be derived, it is first necessary to fully understand the effect of other variables on the SIP response of 
materials. One such variable is the conductivity of the pore fluid. Although for most practical situations the variation of the ionic conductivity with $\sigma_{W}$ is well described by equation (5.2), the manner in which the real and quadrature parts of the surface conductivity vary with pore fluid conductivity is less certain.

\subsection{Measurements}

The two separate samples used to investigate the dependence of SIP spectra on fluid conductivity were sourced from a coastal location on the west coast of the lower North Island of New Zealand. These were of naturally occurring fine sand typical of that found in the shallow coastal aquifers in New Zealand. Particle size analysis showed a grain size distribution with a mean grain diameter of $168 \mu \mathrm{m}$, and a standard deviation of $40 \mu \mathrm{m}$ as shown in Figure 5.1. Sands from further north have a very high titanomagnetite concentration (Nicholson and Fyfe, 1958). Coastal processes mean that sands further south also show considerable magnetic content; with up to $40 \%$ of grains carrying

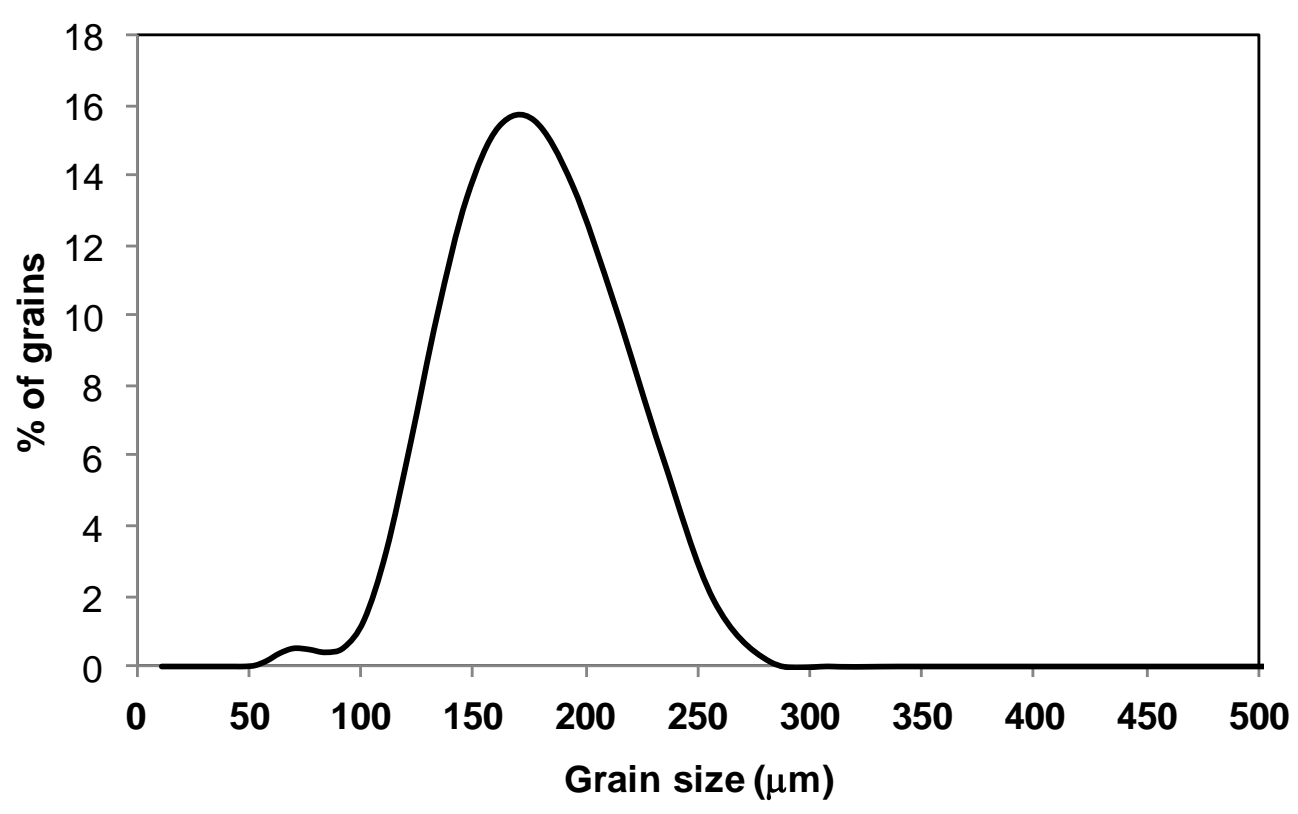

Figure 5.1: Measured grain size distribution for the natural sand used in Samples 1 and 2.

significant magnetic susceptibility (Hawke and McConchie, 2005,2006). Measurements of the mass susceptibility of the sand samples yielded a value of $(1.22 \pm 0.03) \times 10^{-6} \mathrm{~m}^{3} \mathrm{~kg}$ ${ }^{1}$. In comparison clean quartz sand typically has a mass susceptibility of less than $0.6 \mathrm{x}$ $10^{-8} \mathrm{~m}^{3} \mathrm{~kg}^{-1}$ (Hunt et al., 1995). Previous studies (Mansoor and Slater, 2007); Nordsiek and Weller, 2008); (Florsch et al., 2011) have shown that magnetic content in sands/soils leads to an enhanced SIP signal. 
Two separate sample holders of differing internal diameters (49 $\mathrm{mm}$ for Sample 1 and $76 \mathrm{~mm}$ for Sample 2) were used in the measurements. The design of the sample holders was as discussed in Chapter 4. Impedance measurements were made for a range of pore fluid conductivities $\left(\sigma_{W}\right)$ between 12 and $900 \mu \mathrm{S} / \mathrm{cm}$. This covers the range from fresh water to a level of salinity just below the guideline given by the World Health Organization above which water becomes non-potable (WHO, 1996). The conductivity of the fluid was varied by adding $\mathrm{KCl}$ to distilled water. Prior to each measurements fluid of a given conductivity was passed through the sample until the conductivity of the fluid leaving the sample holder stabilized. Each set of measurements (i.e. using a particular sample) started with fluid of the lowest conductivity. The resulting SIP measurements for the two samples, both the magnitude of the bulk conductivity in $\mu \mathrm{S} / \mathrm{cm}$ and the phase difference between current and potential in milliradians, are shown in Figures 5.2 and 5.3.

The SIP measurements show features typical of those observed by previous authors. For a given fluid conductivity, the magnitude of the conductivity shows a slight increase with increasing frequency. This results from the frequency dependence of the surface conductivity. For measurements on both samples, the phase response shows a characteristic shape. At high frequency (approximately $>10 \mathrm{~Hz}$ ) the rapid rise in phase is the result of a combination of instrumentation effects (Breede et al., 2012) and Maxwell-Wagner polarization (Chelidze and Gueguen, 1999). The rise in phase with increasing frequency is also dependent on the magnitude of the current applied to the sample (e.g. the cross-over of phase curves for Sample 2 for fluid conductivities of 80 and $100 \mu \mathrm{S} / \mathrm{cm})$. However, at lower frequencies the phase is found to be independent of current. Below $10 \mathrm{~Hz}$ a maximum in the phase is observed. The magnitude of this is dependent on the pore fluid conductivity with the phase maximum decreasing as the salinity increases. This is largely a result of the increase in ionic conductivity associated with increasing salinity. Apart from for the lowest $(12 \mu \mathrm{S} / \mathrm{cm}$ for Sample 2) and highest (800-900 $\mathrm{\mu S} / \mathrm{cm}$ ) fluid conductivities the phase peak occurs at an approximately constant frequency of about $0.1 \mathrm{~Hz}$. 

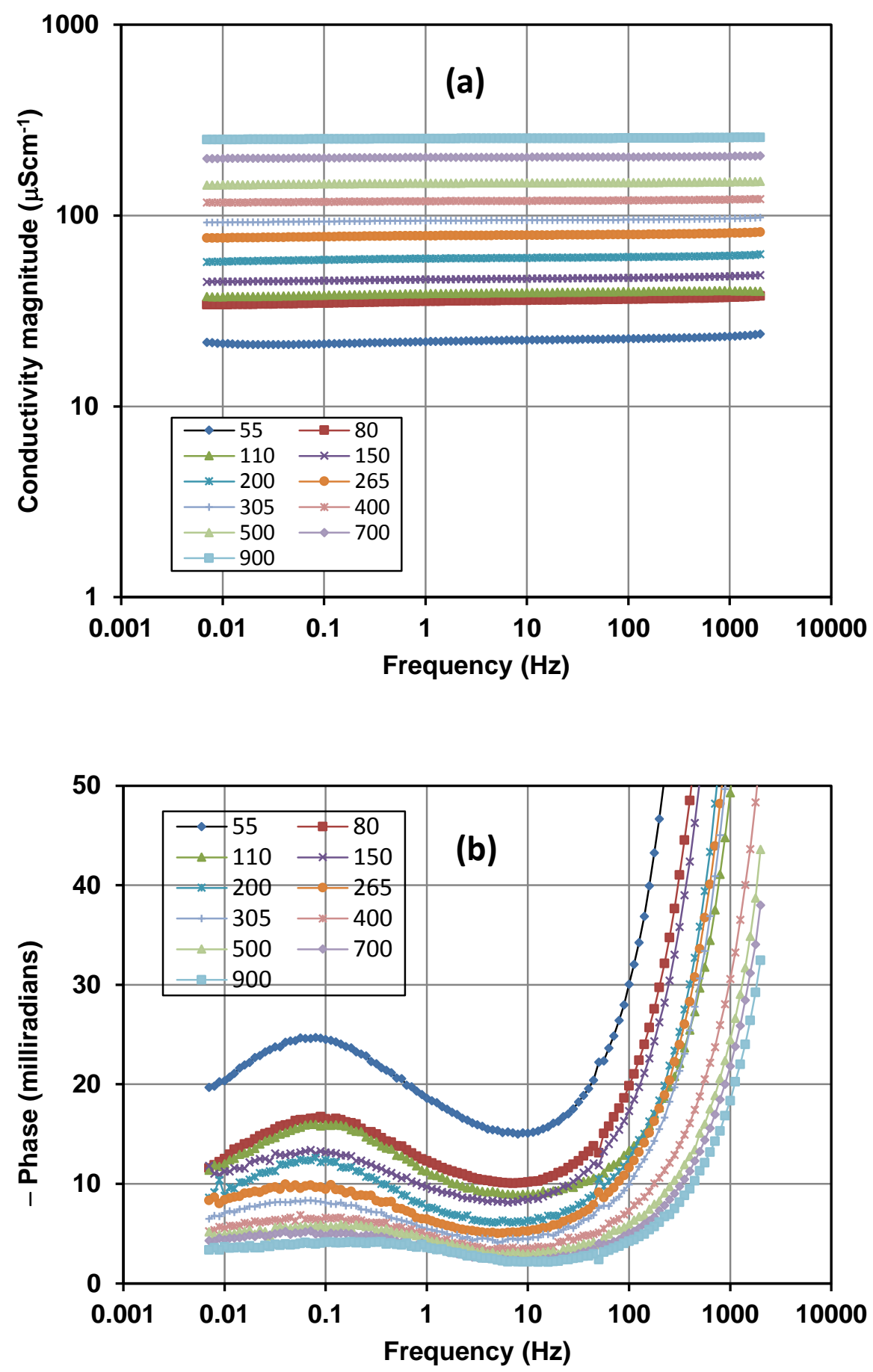

Figure 5.2: SIP measurements on sample 1 for different fluid conductivities (in $\mu$ S $\mathrm{cm}^{-1}$ ) (a) conductivity magnitude, (b) phase. 

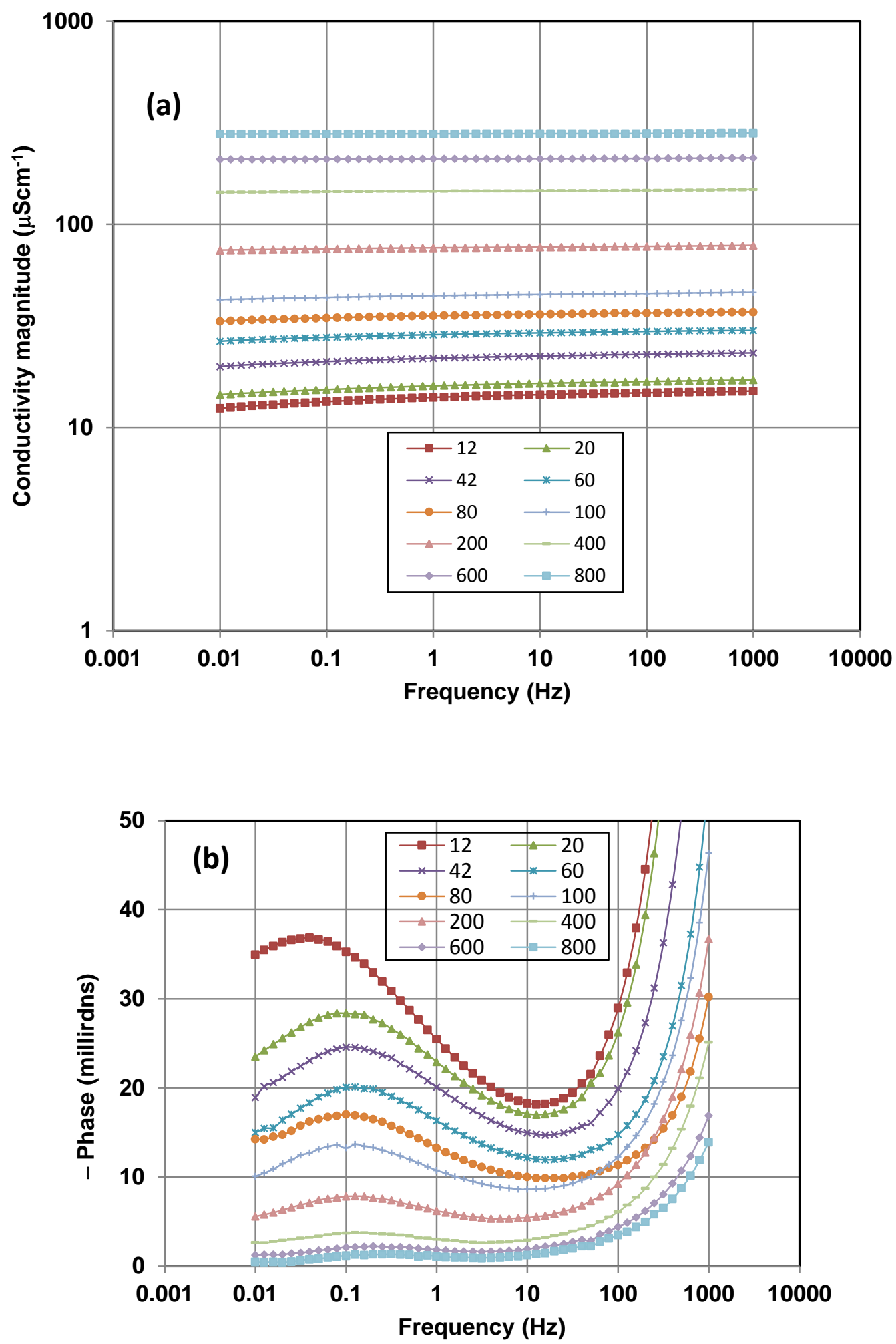

Figure 5.3: SIP measurements on Sample 2 for different fluid conductivities (in $\mu$ S $\mathrm{cm}^{-1}$ ) (a) conductivity magnitude, (b) phase. 


\subsection{Cole-Cole modelling}

As discussed previously, in order to further analyse the data it is necessary to model the measured spectra in terms of a polarization model. Given the likelihood of a range of relaxation times, but to retain a relative simplicity of representation, the Cole-Cole model has been used, for which the bulk complex conductivity of a sample as a function of frequency is given by

$$
\sigma^{*}=\sigma_{0}\left\{1+M\left(\frac{\left(i \omega \tau_{P M}\right)^{c}}{1+(1-m)\left(i \omega \tau_{P M}\right)^{c}}\right)\right\}
$$

Note that equation (5.4) is equivalent to equation (3.22). Here $\sigma_{0}$ is the dc conductivity, $\tau_{P M}$ is the mean relaxation time, the exponent $c$ is a reflection of the spread of relaxation times, and $M$ is the total chargeability which is related to $\sigma_{0}$ and the high frequency conductivity $\sigma_{\infty}$ by

$$
M=1-\frac{\sigma_{0}}{\sigma_{\infty}}
$$

Equation (5.4) is derived by taking the reciprocal of the Cole-Cole model for complex resistivity as introduced by Pelton et al., (1978). The alternative approach is to apply a Cole-Cole model directly to the complex conductivity, in which case $\sigma^{*}$ is represented by

$$
\sigma^{*}=\sigma_{\infty}+\frac{\sigma_{0}-\sigma_{\infty}}{1+\left(i \omega \tau_{C C}\right)^{c}}
$$

Equation (5.6) is the formulation used by, for example, (Revil and Florsch, 2010). Although the relaxation times in each formulation are different (Tarasov and Titov, 2013) they are related by

$$
\tau_{C C}=\tau_{P M}(1-M)^{1 / c}
$$

As the high frequency rise in the SIP phase is not related to the surface polarization effects of interest, and requires a more complex model to adequately represent it, equation (5.4) has been used to fit the measurements on both samples only in the frequency range from 0.01-3 Hz. Modelling was carried out in MS Excel using the data analysis package Solver. The results of this fitting, in terms of the variations in parameters $\sigma_{0}, \tau_{C C}, M$ and $c$ with fluid conductivity are shown in Figures 5.4, 5.5, 5.6 and 5.7. The derived SIP parameters and $\tau_{C C}$ calculated using equation (5.7) are listed in 


\begin{tabular}{|c|c|c|c|c|c|c|}
\hline & $\begin{array}{c}\sigma_{w} \\
\left(\mu \mathrm{Scm}^{-1}\right)\end{array}$ & $\begin{array}{c}\sigma_{o} \\
\left(\mu \mathrm{Scm}^{-1}\right)\end{array}$ & $\tau_{P M}(\mathbf{s})$ & $M$ & $c$ & $\tau_{C C}(\mathbf{s})$ \\
\hline \multirow{11}{*}{ Sample 1} & 55 & 19.7 & 2.61 & 0.146 & 0.379 & 1.72 \\
\hline & 80 & 33.2 & 1.87 & 0.09 & 0.424 & 1.5 \\
\hline & 110 & 36.6 & 1.99 & 0.082 & 0.445 & 1.64 \\
\hline & 150 & 43.5 & 2.77 & 0.082 & 0.376 & 2.22 \\
\hline & 200 & 56.6 & 2.38 & 0.06 & 0.471 & 2.09 \\
\hline & 265 & 75 & 3.18 & 0.055 & 0.424 & 2.78 \\
\hline & 305 & 90.7 & 2.88 & 0.046 & 0.429 & 2.58 \\
\hline & 400 & 115.6 & 2.36 & 0.039 & 0.413 & 2.14 \\
\hline & 500 & 143.7 & 2.08 & 0.035 & 0.397 & 1.9 \\
\hline & 700 & 197.2 & 2.66 & 0.03 & 0.414 & 2.47 \\
\hline & 900 & 248.2 & 1.5 & 0.029 & 0.355 & 1.39 \\
\hline \multirow{10}{*}{ Sample 2} & 12 & 11.4 & 7.36 & 0.231 & 0.347 & 3.46 \\
\hline & 20 & 14 & 2.15 & 0.17 & 0.373 & 1.3 \\
\hline & 42 & 19.5 & 1.64 & 0.143 & 0.388 & 1.1 \\
\hline & 60 & 26.3 & 1.34 & 0.111 & 0.411 & 1.01 \\
\hline & 80 & 32.8 & 1.94 & 0.101 & 0.387 & 1.47 \\
\hline & 100 & 42.1 & 1.44 & 0.077 & 0.41 & 1.19 \\
\hline & 200 & 74.1 & 1.21 & 0.043 & 0.424 & 1.09 \\
\hline & 400 & 143.6 & 1.01 & 0.02 & 0.431 & 0.961 \\
\hline & 600 & 208.9 & 0.62 & 0.01 & 0.492 & 0.605 \\
\hline & 800 & 278.3 & 0.46 & 0.005 & 0.648 & 0.453 \\
\hline
\end{tabular}

Table 5.1: Cole-Cole parameters $\sigma_{0}, \tau_{P M}, \mathrm{M}$ and $\mathrm{c}$ from fitting the measured SIP spectra for samples with different fluid conductivity $\sigma_{w}$ with equation (5.4). $\tau_{C C}$ is the relaxation time corresponding to equation (5.6) calculated from equation (5.7). The uncertainty in $\sigma_{w}$ is $\pm 1 \mu \mathrm{Scm}^{-1}$. The uncertainty in estimated Cole-Cole parameters is $\pm 7 \%$. 


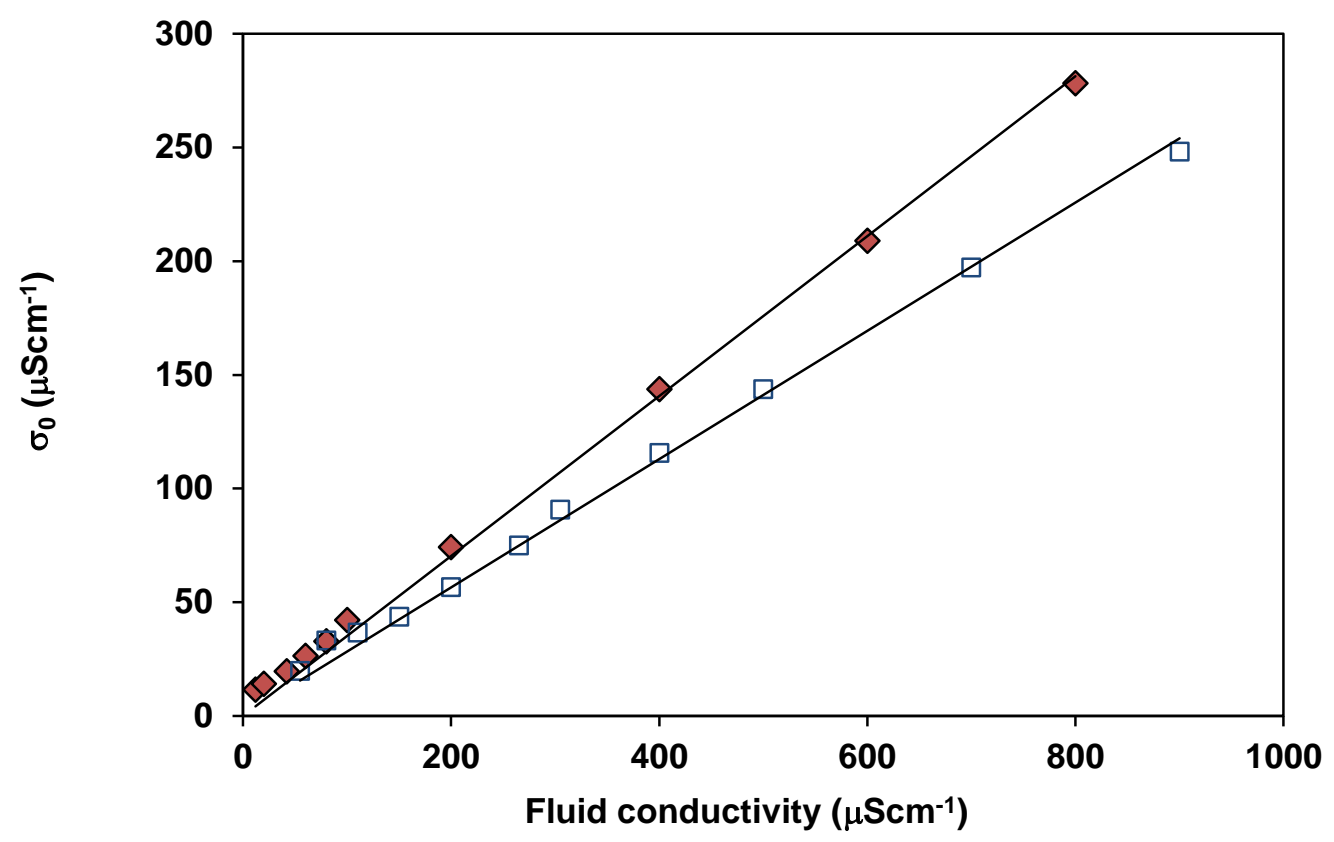

Figure 5.4: Variation of Cole-Cole parameter DC resistivity $\left(\sigma_{0}\right)$ with fluid conductivity Sample 1 - squares; Sample 2 diamonds.

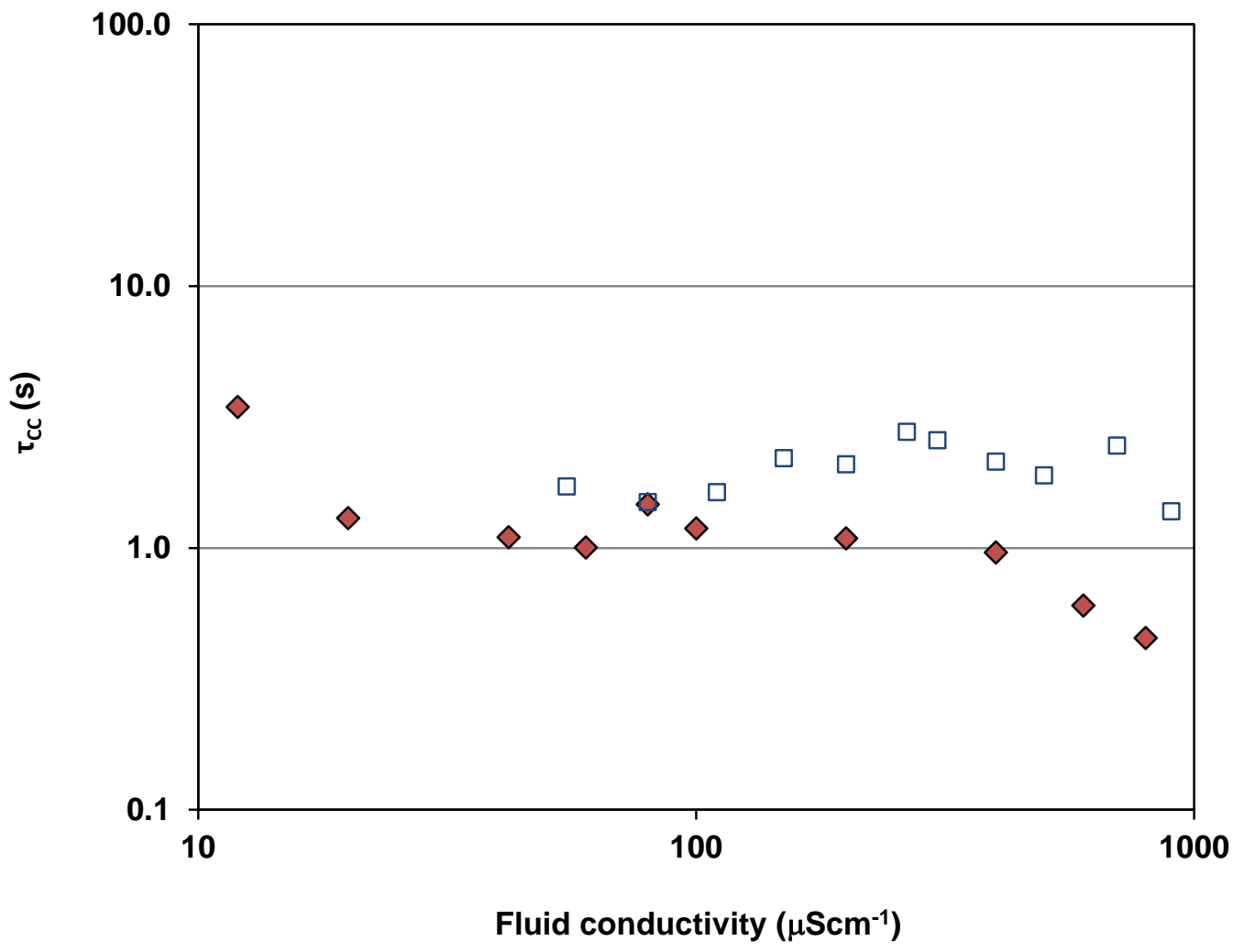

Figure 5.5: Variation of Cole-Cole parameter relaxation time $\left(\tau_{C C}\right)$ with fluid conductivity Sample 1 -squares; Sample 2 diamonds. 


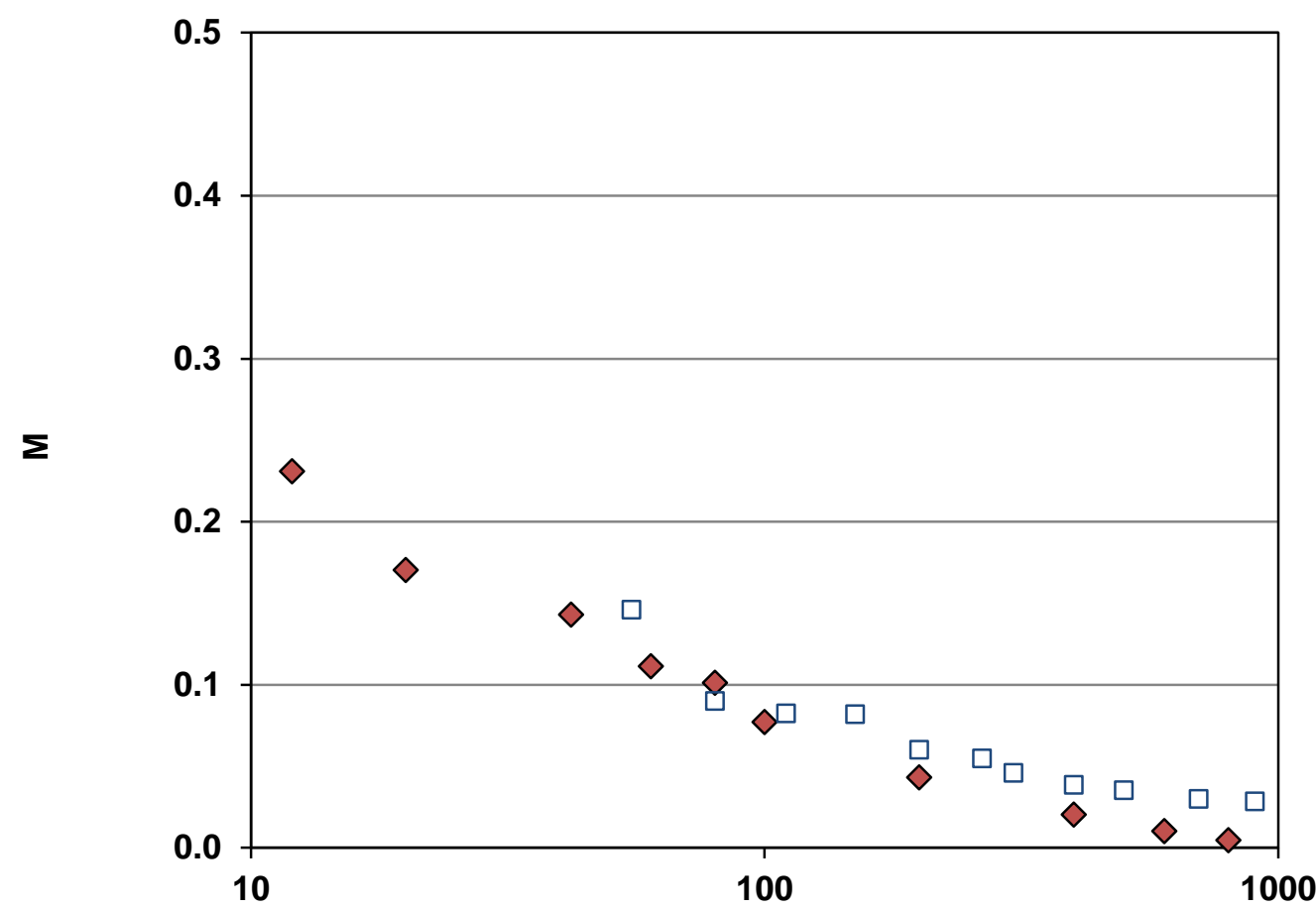

Fluid conductivity $\left(\mu \mathrm{Scm}^{-1}\right)$

Figure 5.6: Variation of Cole-Cole parameter chargeability $(M)$ with fluid conductivity Sample 1 - squares; Sample 2 diamonds.

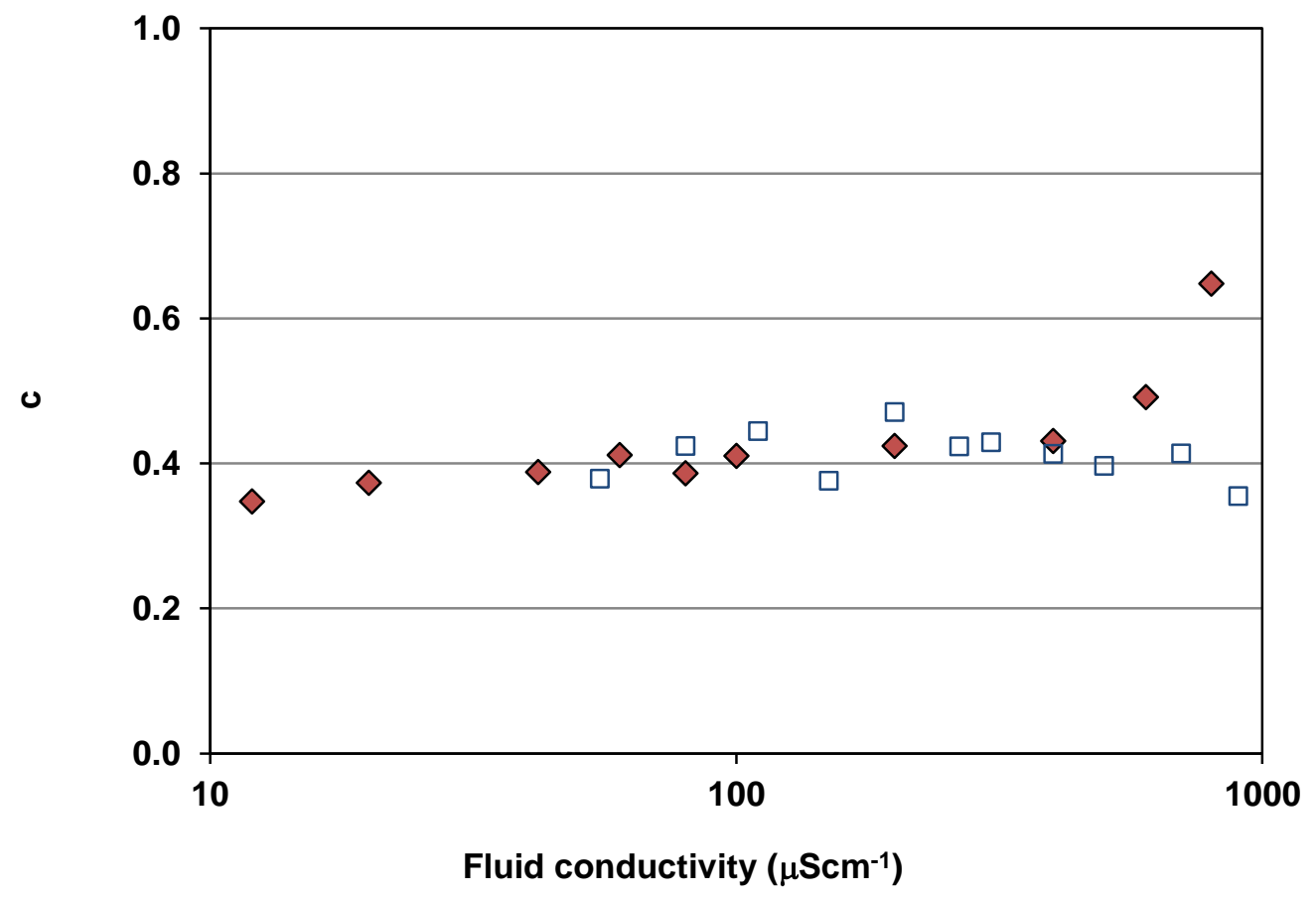

Figure 5.7: Variation of Cole-Cole parameter c with fluid conductivity Sample 1 squares; Sample 2 diamonds. 
Table 5.1.

The linearity of the plot of $\sigma_{0}$ as a function of the fluid conductivity $\sigma_{w}$ (Figure 5.4) allows estimation of the formation factor $F$ for each of the samples, and gives values of $F=$ $3.54 \pm 0.02$ for Sample 1, and $F=2.84 \pm 0.02$ for Sample 2. Assuming the validity of equation (5.2) the measured value of porosity of the Sample 2 of $47 \pm 1 \%$ allows the cementation exponent $m$ to be calculated as $1.37 \pm 0.04$. Given the broadness of the phase peaks shown in Figures 5.2(b) and 5.3(b), the results shown in Figure 5.5 demonstrate that the relaxation time is not only independent of $\sigma_{w}$, but is also essentially the same for both samples. Similarly, both sets of data show a similar monotonic decrease in chargeability with increasing fluid conductivity (Figure 5.6), and a constant value for the exponent $c$ (Figure 5.7). Several authors (Leroy et al., 2008); (Revil and Florsch, 2010); (Breede et al., 2012) have previously reported that the relaxation time is related to the square of the grain diameter. Thus, given that the data are all measured on the same sand, the independence of $\tau_{C C}$ with fluid conductivity is unsurprising. Similarly, the constancy of $c$, related to the spread of relaxation times, is expected. As for the decrease in SIP phase with increasing fluid conductivity, the decrease in $M$ can be explained by the increasing dominance of ionic conductivity over polarization effects as the fluid becomes increasingly conductive. As the Cole-Cole modelling provides a value for the dc conductivity, assuming that the ionic conductivity is much greater than the DC surface conductivity, equation (5.3) may be rewritten as

$$
\sigma^{*}=\sigma_{0}+\sigma_{\text {surf }}^{\prime}+i \sigma_{\text {surf }}^{\prime \prime}
$$

and thus, for any fluid conductivity, $\sigma_{\text {surf }}^{\prime}$ may be estimated as

$$
\sigma_{\text {surf }}^{\prime}=\operatorname{Re}\left(\sigma^{*}\right)-\sigma_{0}
$$

The imaginary part of $\sigma^{*}$ gives $\sigma_{\text {surf }}^{\prime \prime}$. Plots of $\sigma_{\text {surf }}^{\prime}$ and $\sigma_{\text {surf }}^{\prime \prime}$ as functions of $\sigma_{w}$ at a frequency of $0.1 \mathrm{~Hz}$ (the phase peak), and the variation in $\sigma_{0}\left(\sigma_{\text {ionic }}\right)$ with $\sigma_{w}$ are shown in Figure 5.8.

For Sample 1 values of $\sigma_{\text {surf }}^{\prime}$ and $\sigma_{\text {surf }}^{\prime \prime}$ show a gradual increase with fluid conductivity. At lower fluid conductivities the same trend is shown by values for Sample 2. However, for Sample 2 the two highest fluid conductivity measurements suggest that a maximum in both the real and imaginary parts of $\sigma_{\text {surf }}$ exist. Although this is a similar behaviour 
to that noted by Slater and Lesmes, (2002), who reported a broad maximum in $\sigma_{\text {surf }}^{\prime \prime}$ over a fluid conductivity range of $10-2000 \mu \mathrm{Scm}^{-1}$, and further measurements at high conductivity are clearly desirable, the same measurements also lead to anomalously high values of $\tau_{C C}$, and low values of $c$ compared to the other measurements (Figure 5.7). These may result from difficulty in fitting a distinct model to these measurements due to the very low phase values and extremely broad maximum in the phase spectrum (Figure 5.2(b)).

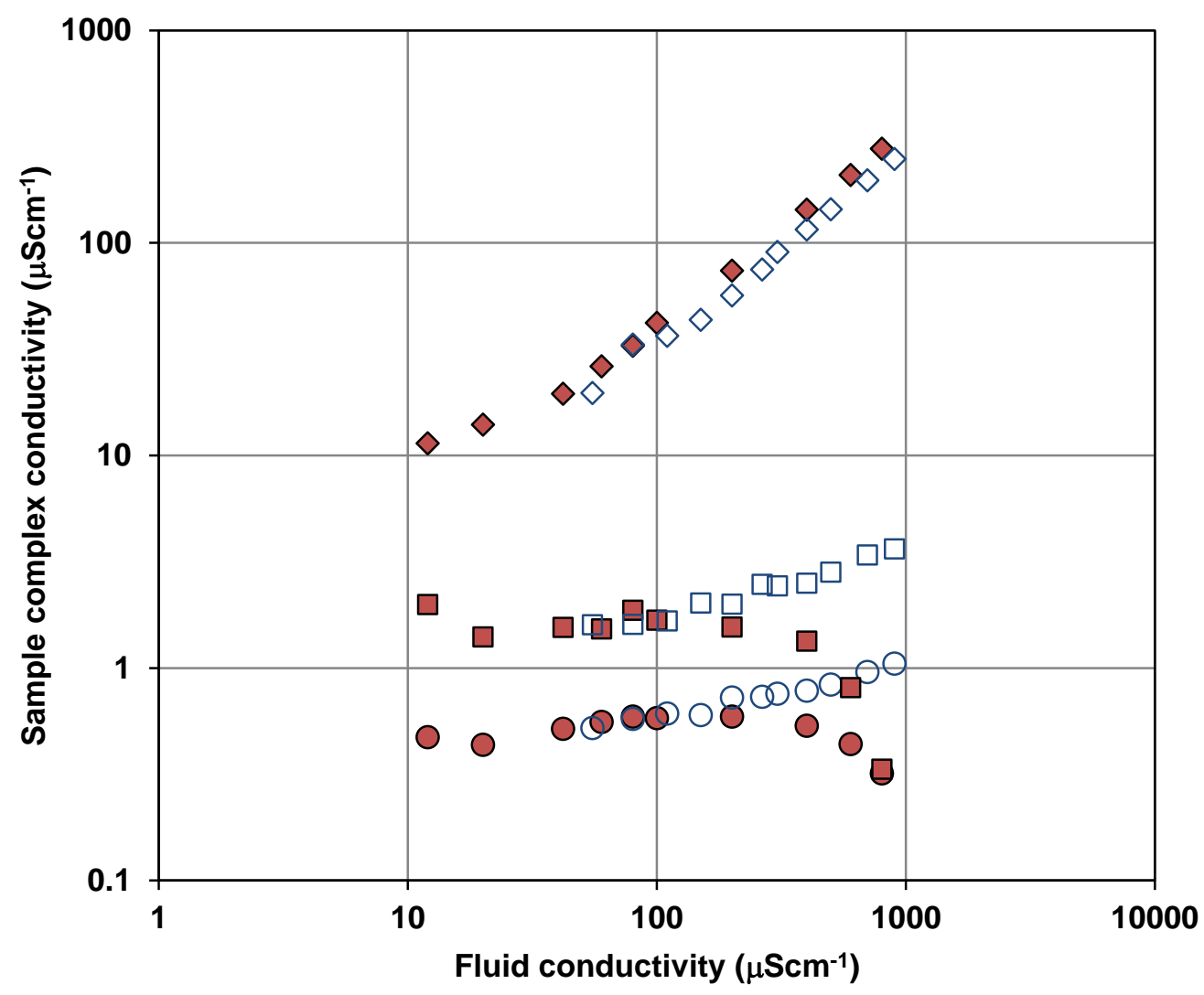

Figure 5.8: Variation with fluid conductivity $\left(\sigma_{w}\right)$ of the in-phase ( $\sigma_{\text {surf }}^{\prime}$ - squares) and quadrature-phase ( $\sigma_{\text {surf }}^{\prime \prime}$ - circles) parts of the surface conductivity. Diamonds show the variation of the ionic conductivity $\left(\sigma_{\text {ionic }}\right)$. Open symbols are sample 1 shaded symbols are sample 2 . The uncertainty in $\sigma_{w}$ is $\pm 1 \mu \mathrm{Scm}^{-1}$. The uncertainty in estimated $\sigma_{\text {surf }}^{\prime}$ and $\sigma_{\text {surf }}^{\prime \prime}$ is $\pm 7 \%$.

\subsection{Conclusion}

The results of the above set of measurements on the influence of pore fluid conductivity on SIP spectra show that the variation of Cole-Cole relaxation time with pore fluid conductivity is very small for fluid conductivities in the range $100 \mu \mathrm{Scm}^{-1}$ to $2000 \mu \mathrm{Scm}^{-}$ 
1. Data from table 5.1 suggests that there is a possibility of significant variation of relaxation time constant below $100 \mu \mathrm{Scm}^{-1}$ and above $2000 \mu \mathrm{Scm}^{-1}$. Such variation of Cole-Cole relaxation time constant has been reported by Munch (2009).

Further investigations were not conducted as those pore fluid conductivities were outside the range of interest for the current project. It should be noted that it may be difficult to stabilise the pore fluid conductivity to a particular value at conductivities below $100 \mu \mathrm{Scm}^{-1}$ for natural samples that have minerals dissolving into the pore fluid. Such small variations in Cole-Cole relaxation constant are insignificant in the case of field measurements. But they may have relevance when it comes to the explanation of the phenomenon of SIP. Further observations, related to the samples presented in chapter 6 , reiterate the possibility of a noticeable variation of relaxation time constant at pore fluid conductivities less than $100 \mu \mathrm{Scm}^{-1}$. 


\section{Variation of hydraulic conductivity with grain size.}

\subsection{Introduction}

Groundwater is a fragile natural resource that demands careful handling to maintain sustainable use. The development, management and protection of ground water resources requires a good knowledge of the transmissivity, storage coefficient and hydraulic conductivity. These parameters help to assess the potential yield of aquifers over a long period of exploitation. The hydraulic conductivity is of great importance in geotechnical fields like determination of seepage losses, settlement computation, stability analyses, and virtually every other hydrogeologic investigation.

The hydraulic conductivity is the most important hydraulic property of an aquifer. But determination of hydraulic conductivity is challenging because of the size of aquifers, and the spatial variation of hydraulic conductivity. The methods of determination of hydraulic conductivity include field measurements like pumping tests, laboratory measurements on core samples, and analysis of grain size distribution. Each of these methods has its advantages and disadvantages. Field measurements are costly and time consuming and cover only a very small portion of the aquifer. Measurements on core samples and other samples collected from the field provide only localised information. Hydraulic conductivity measurements can be performed on repacked or core sediments in the laboratory, but such measurements may not yield accurate measurements as sediment properties that affect hydraulic conductivity, such as grain cohesion, density and layering may be disturbed during laboratory measurements (Eggleston and Rojstaczer, 2001). In geophysical surveys, although there may be no direct relationship between the geophysical quantity under study and the hydraulic conductivity, there is the advantage that they can cover a large area at reduced cost and time.

\subsection{Permeability from grain size distribution.}

It has been widely recognised that the hydraulic conductivity of a porous medium is controlled by the grain size distribution. There exist various models that try to predict the permeability from the grain size distribution. These consist of empirical relationships, capillary models, statistical models, and hydraulic radius theories. These 
predictions are usually based on properties of the medium such as the porosity, the size of the pores, the tortuosity and the pore connectivity (Chapuis and Aubertin, 2003). Rosas et al., (2014) tabulate 20 such equations that are in use. In all these equations the hydraulic conductivity is given by a general form

$$
k=c d^{n}
$$

where $k$ is the intrinsic permeability, $c$ is a dimensionless constant representing the shape and packing of grains, $d$ is the representative diameter of the grains and $n$ is an exponent often equal to or very close to 2 .

The representative grain diameter used in various models include: (1) the diameter at which $10 \%$ of the particles are smaller $\left(d_{10}\right) ;(2)$ the median diameter $\left(d_{50}\right) ;(3)$ the geometric mean; and, (4) the harmonic mean (Koltermann and Gorelick, 1995).

Attempts have been made to accommodate the effects of grain size distribution with a third term that contains the standard deviation of grain size distribution (Masch and Denny, 1966). Among these equations the Kozeny-Carman equation has been successful in the case of soils and sediments (Porter et al., 2013), (Koltermann and Gorelick, 1995) and takes the form

$$
k=\frac{d^{2} \Phi^{3}}{180(1-\Phi)^{2}}
$$

where $k$ is the intrinsic permeability, $d$ is a representative grain diameter, and $\Phi$ is the effective porosity. The intrinsic permeability is related to the hydraulic conductivity as shown in equation (4.3).

When using the Kozeny-Carman formula the choice of the representative grain diameter is critical to the successful prediction of the permeability (Boadu, 2000). In case of bimodal mixtures of grains, the effective value of $d$ is computed as follows. When there are not enough finer grains to occupy the pores formed between the larger grains $d$ is taken as the geometric mean of the diameters of the finer and coarser grains weighted by their fraction of the mixture. If there are enough finer grains to occupy all coarse pores, then the representative diameter is calculated as the harmonic mean of the diameters of finer and coarser grains weighed by their fractions of the mixture (Porter et al., 2013). 


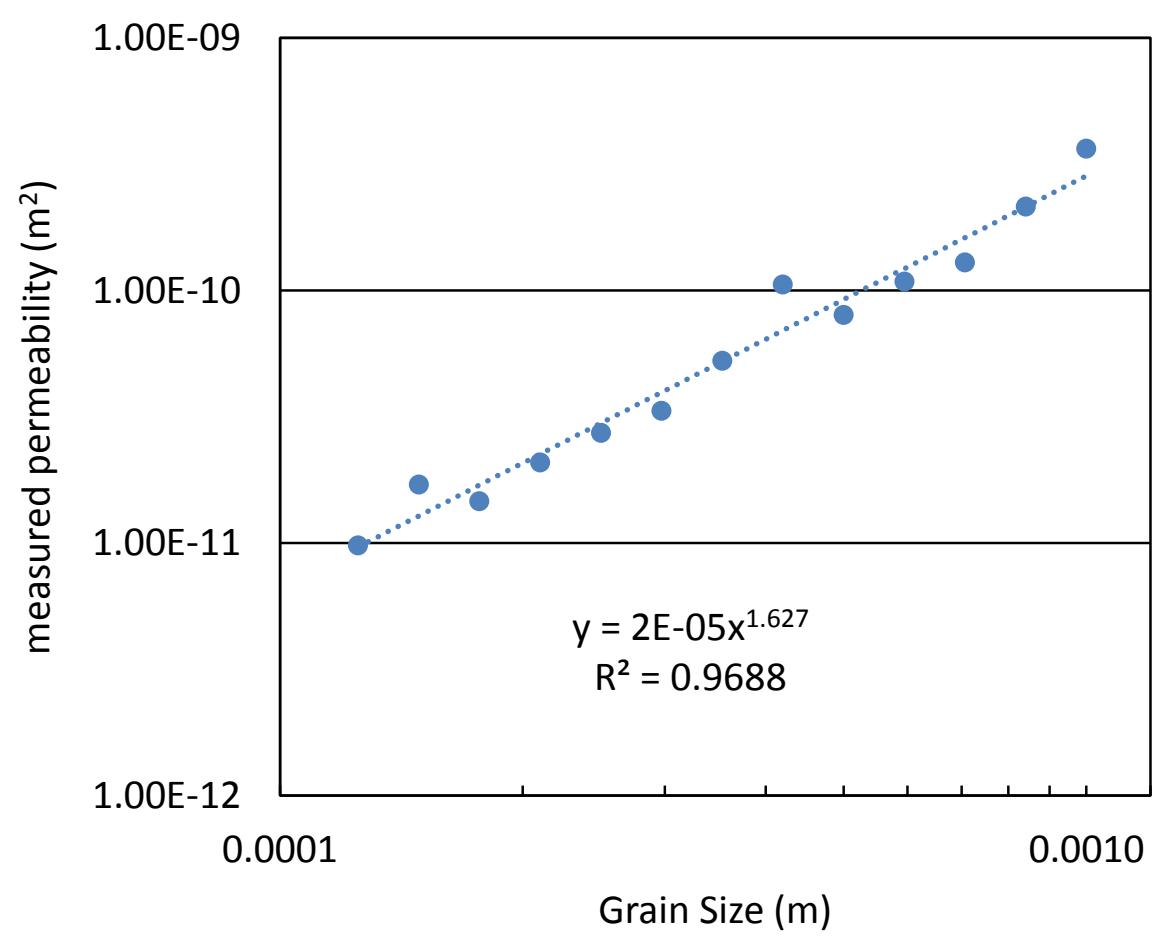

Figure 6.1: Measured permeability plotted against grain size for series 1 samples

During this project hydraulic conductivity and SIP measurements were conducted on the two series of samples that are made up sand sieved using sieves that differ by $0.25 \varphi$. As the sieves differ $0.25 \varphi$, the smallest grain and largest grain of sand differ in size by about $16 \%$ only. Therefore, the grain size can be taken as equal to the sieve mesh size. Figure 6.1 shows a plot of the measured permeability against the grain size of the series 1 samples which are explained in section 6.4. When the data are fitted to the model given by equation (6.1) a values of $1.62 \pm 0.09$ and $(2.16 \pm 0.15) \times 10^{-5}$ respectively are found for the constants $n$ and $c$

Figure 6.2 shows a plot of the measured permeability and the permeability estimated using equation (6.2) plotted against the grain size. It can be seen that equation (6.2) overestimates the permeability by a factor of about 3. Figure 6.2, however, clearly indicates that the samples under study shows a permeability variation as expected and hence they are suitable for investigating the relationship between permeability and grain size, or any other proxy that can be used to estimate permeability like Cole-Cole relaxation time. 


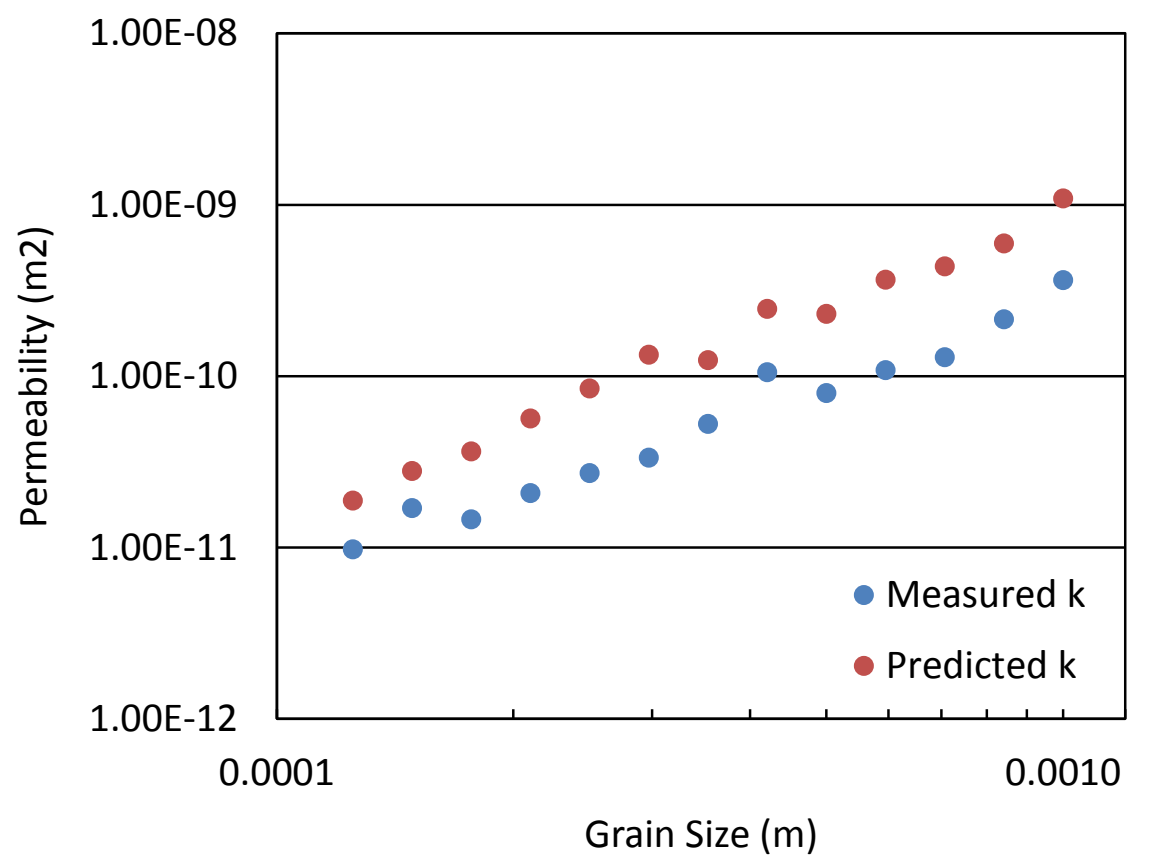

Figure 6.2: Measured permeability and permeability predicted using KozenyKarman equation plotted against grain size for Series 1 samples.

\subsection{SIP measurements as a predictor of permeability}

There have been many attempts to use electrical methods for hydrogeophysical applications. The similarity between the flow of water and current through a porous medium encourages the use electrical methods for hydraulic conductivity/permeability estimation. Slater (2007) gives a summary of the efforts to relate electrical properties to hydrological properties like hydraulic conductivity. The conductivity of a saturated porous material is a complex quantity - that is, when an electric current is injected into such a material a measured potential difference has both in-phase and quadraturephase components

$$
\sigma^{*}=\sigma^{\prime}+i \sigma^{\prime \prime}
$$

The real part of the complex conductivity $\left(\sigma^{\prime}\right)$ is the result of a combination of ionic conductivity through the pore fluid and charge migration along grain/pore surfaces. The imaginary part $\left(\sigma^{\prime \prime}\right)$ is the result of polarization processes resulting from the existence of an electrical double layer adjacent to the grain/pore surfaces. Equation (6.3) may also therefore be expressed as

$$
\sigma^{*}=\sigma_{\text {ionic }}+\sigma_{\text {surf }}=\sigma_{\text {ionic }}+\sigma_{\text {surf }}^{\prime}+i \sigma_{\text {surf }}^{\prime \prime}
$$


where the surface conductivity $\left(\sigma_{\text {surf }}\right)$ is complex.

As the different components of the complex electrical conductivity are controlled by different mechanisms that operate during the flow of current, they provide information about different properties of the porous medium as shown in Figure 6.3. Compared to other electrical methods, SIP has promise to provide information more specific to a hydraulic length scale that controls the hydraulic conductivity.

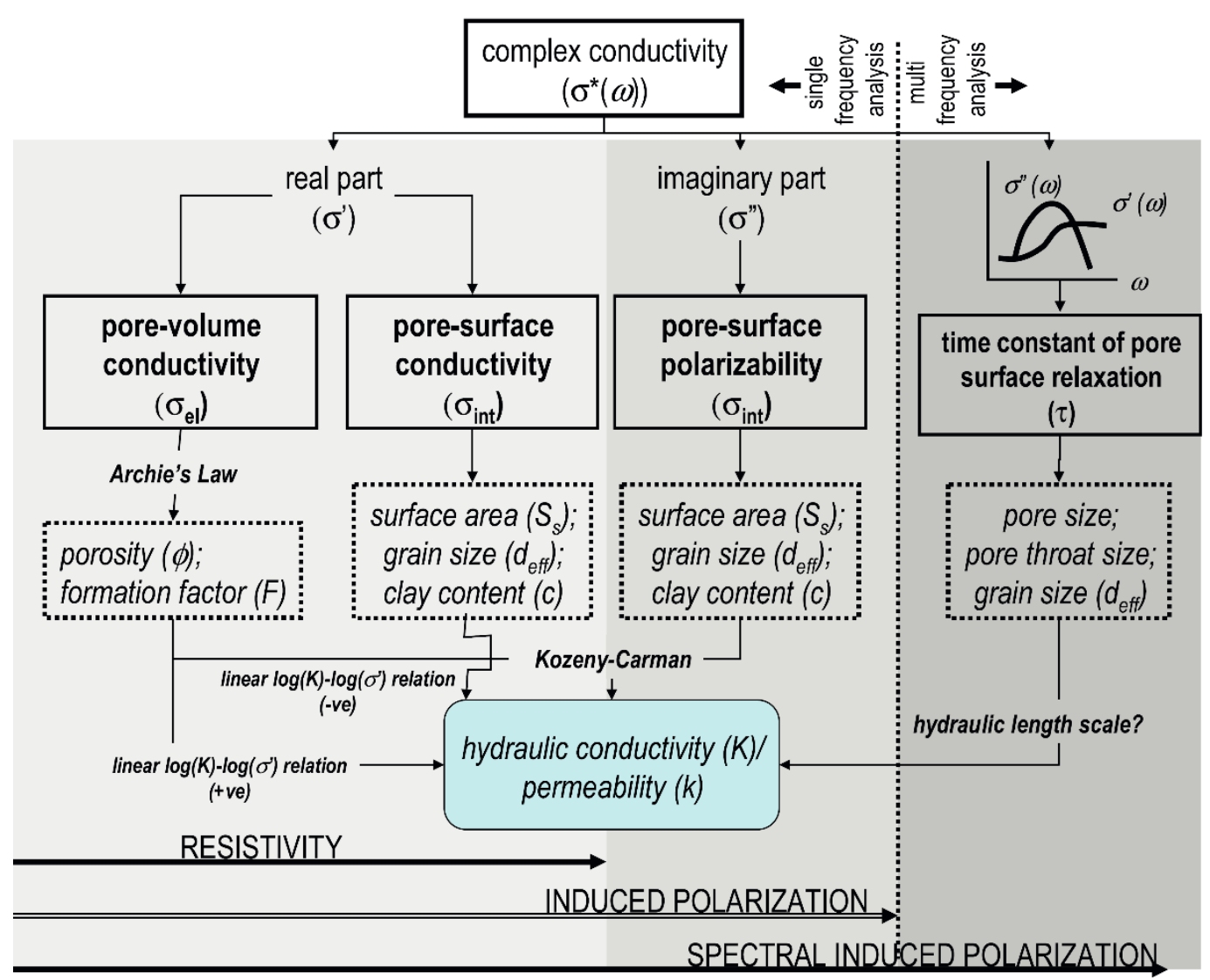

Figure: 6.3: Flowchart summarising efforts to predict hydraulic conductivity (K) from petrophysical relationships established for electrical measurements (from Slater, 2007).

The phase part of the complex conductivity of a porous medium when plotted against frequency leads to a characteristics peak at lower frequencies as shown in Figure 6.4. This has been noted by many authors (Binley et al., 2005); (Koch et al., 2012); (Nordsiek and Weller, 2008); (Revil et al., 2013); (Weller et al., 2011). The knowledge that polarization processes that lead to this characteristic peak are taking place at grain/pore surfaces has led to the belief that such measurements may be a suitable proxy for the 
hydraulic properties of a porous material. This has generated a renewed interest in using SIP for permeability prediction. (Avellaneda and Torquato, 1991); (Borner et al., 1996); (Hordt at al., 2007); (Koch et al., 2012); (Revil and Florsch, 2010); (Revil at al., 2012a); (Slater, 2007); (Slater and Lesmes, 2002); (Tong et al., 2006a;b); (Tong and Tao, 2008); (Zisser et al., 2010a;b).

As detailed in Chapter 3 there are many possible mechanisms that contribute to the SIP response. While authors differ on the exact nature of polarization, it is generally accepted that at least in unconsolidated sediments like sands it is the polarization of the electrical double layer that gives rise to the characteristic SIP response as shown in Figure 6.4. A relationship between SIP spectra and hydraulic properties is established by fitting the observed spectra to one of the many available phenomenological models as explained in Chapter 3.

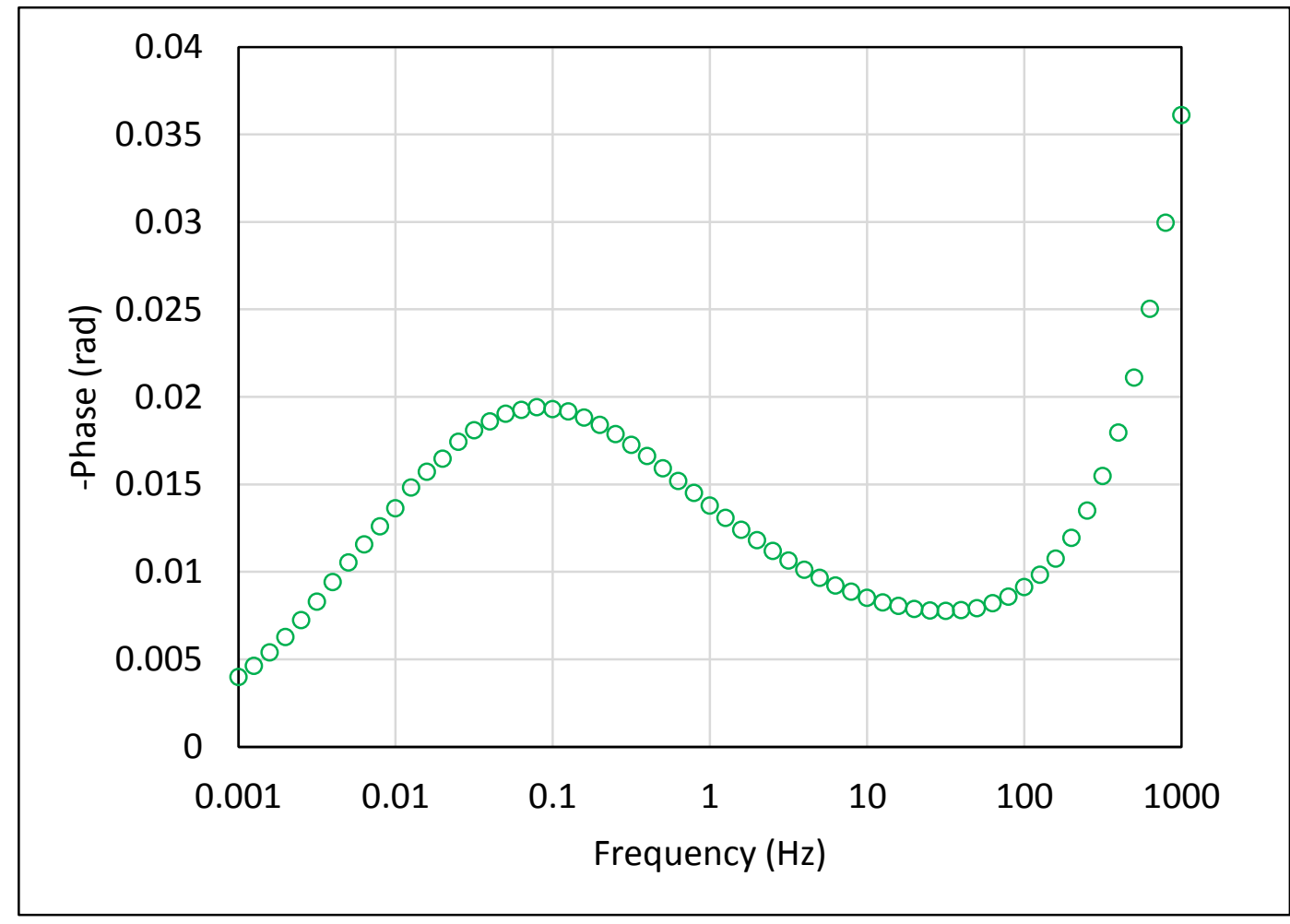

Figure 6.4: Typical example of SIP phase spectrum showing a peak in the phase of the complex conductivity at low frequency.

In the Cole-Cole model the complex conductivity is represented by

$$
\sigma^{*}=\sigma_{o}\left\{1+\frac{M}{1-M}\left(1-\frac{1}{1+\left(i \omega \tau_{C C}\right)^{c}}\right)\right\}
$$


(Tarasov \& Titov, 2013) where $\sigma_{o}$ is the dc conductivity, $\omega$ is the angular frequency (= $2 \pi f), \tau_{c c}$ is the Cole-Cole relaxation time, $c$ the Cole-Cole exponent, and $M$ is the chargeability, related to both $\sigma_{o}$ and the high frequency conductivity $\sigma_{\infty}$ by $M=\left(\sigma_{\infty}\right.$ $\left.\sigma_{o}\right) / \sigma_{\infty}$. The relaxation time, $\tau_{C c}$, is related to the frequency at which the peak in the phase spectrum occurs. It is this model for complex conductivity which is used in this thesis.

The samples under investigation are prepared by tight packing of very well sieved sand into a cylindrical sample holder. The porosity of all the samples is around $40 \%$. Two relationships connecting the SIP parameter $\tau_{C C}$ and permeability have recently been investigated by Revil et al. (2012). Given the conditions under which these relationships were developed, and the nature of the samples in this study, these relationships appear promising as possible predictors of permeability. These two relationships are based on the suggestion by Johnson et al. (1986) that the permeability $(k)$ of a porous material can be related to the dynamic pore radius $(\Lambda)$, a weighted pore volume to surface area ratio, and the electrical formation factor $(F)$ by the approximation that

$$
k \approx \frac{\Lambda^{2}}{8 F}
$$

The formation factor represents the ratio of the pore fluid conductivity to the dc bulk conductivity of the porous material and, through Archie's Law (Archie, 1942), for a saturated material is related to the porosity $(\phi)$ by

$$
F=\frac{\sigma_{f}}{\sigma_{0}}=\phi^{-m}
$$

where $m$, known as the Archie's exponent or cementation exponent, takes particular values for different types of consolidated or unconsolidated materials. An equation similar to (6.6) was developed by Avellaneda and Torquato (1991)

$$
k=\frac{L^{2}}{8 F}
$$

where $k$ is the permeability $L$ is a parameter having dimensions of length and $F$ is the electrical formation factor. The parameter $L$ is related to eigen function expansion coefficients of the electric field in the pore space. For porous rocks when averaged over the distribution of pore aspect ratios it turns out that $\frac{L}{\Lambda} \approx 1$ 
According to Johnson et al. (1986) the bulk conductivity of a porous material saturated with fluid of conductivity $\sigma_{f}$ can be written in the form

$$
\sigma=\frac{1}{F}\left(\sigma_{f}+\frac{2}{\Lambda} \Sigma_{S}\right)
$$

where $\Sigma_{S}$ is the surface conductance. Taking the high salinity limit and using a binomial expansion of the bulk conductivity equation proposed by Bussian (1983), Revil \& Cathles (1999) suggested that the bulk conductivity as given by equation (6.9) can also be written as

$$
\sigma=\frac{1}{F}\left(\sigma_{f}+m(F-1) \sigma_{s}\right)
$$

where $m$ is the Archie's exponent and $\sigma_{s}$ is the surface conductivity of the grains. Based on the suggestion by Revil \& Glover (1997) that $\sigma_{s}=4 \Sigma_{S} / d$ where $d$ is the diameter of a spherical grain, equation (6.10) can be written as

$\sigma=\frac{1}{F}\left(\sigma_{f}+m(F-1) 4 \frac{\Sigma_{s}}{d}\right)$

Now equating equation (6.9) and (6.11) $\Lambda$ can be expressed in terms of $d, m$ and $F$ as

$\frac{1}{\Lambda}=2 m \frac{(F-1)}{d}$

Equation (6.12) along with (6.6) allows the permeability to be expressed in terms of the grain diameter and parameters related to the bulk electrical conductivity

$$
k=\frac{d^{2}}{32 m^{2} F(F-1)^{2}}
$$

The polarization process from which the SIP response is believed to be originating is characterised by the relaxation time constant which is related to the grain/pore size. Such a relationship between the relaxation time constant and grain size is given for colloids by Schurr (1964) as

$$
\tau=\frac{d^{2}}{8 D}
$$


where $d$ is the dimater of the grain and $D$ is the diffusion coefficient of ions in the fluid that saturates the grains.

The work of Leroy et al. (2008) and Revil \& Florsch (2010) suggests that equation (6.14) can be adopted for the relaxation mechanism in porous media also, and the relaxation time is then identified with the Cole-Cole relaxation time $\tau_{c c}$, and the diffusion coefficient with that of the diffusion coefficient $\left(D_{i}\right)$ of counter ions attached to the grain surface in the Stern layer.

$$
\tau_{C C}=\frac{d^{2}}{8 D_{i}}
$$

Combining equation (6.15) with (6.13) gives an expression that relates permeability to the Cole-Cole relaxation time of the SIP phase

$$
k=\frac{\tau_{C C} D_{i}}{4 m^{2} F(F-1)^{2}}
$$

The circumstances under which the above equation was developed indicate that it may be reliable only for unconsolidated sediments. A more promising relationship for consolidated sediments may be the one suggested by Revil et al. (2012).

$$
\tau_{C C}=\frac{\Lambda^{2}}{2 D_{i}}
$$

To arrive at the equation (6.17) Revil et al. (2012) hypothesised that the dynamic pore radius can be equated to $d / 2$. The suggestion by Binley et al. (2010) that for consolidated materials it could be the pore size rather than the grain size that could be the controlling factor as far as the Cole-Cole relaxation time is concerned supports the use of equation (6.17).

Equation (6.17) and (6.6) together provides an alternative predictor for permeability as

$$
k=\frac{\tau_{C C} D_{i}}{4 F}
$$

Equations (6.16) and (6.18) can be considered to be based on the assumptions that the relaxation time is dependent on either the grain size or the pore size 
Not all porous materials display the characteristic peak at low frequencies. For such samples the surface conductivity $\left(\sigma_{\text {surf }}\right)$, or the imaginary part of the conductivity $\left(\sigma^{\prime \prime}\right)$, measured at a certain frequency has been used for permeability prediction. The frequency typically used is $1 \mathrm{~Hz}$. This approach is seen in the work of Börner et al., (1996), and Weller et al. (2010). They consider that $\sigma^{\prime \prime}$ is related to the specific internal surface ( $S_{\text {por }}-$ the internal pore surface area per unit volume of material). Weller et al. (2015) have summarized this approach to predicting permeability and suggested fitting measured data with relationships of the form

$$
k=\frac{a}{F^{b} S^{c}}
$$

where $a$ is a constant, $b$ and $c$ are empirically determined exponents. $S$ may be either $\sigma_{\text {surf }}$ or $\sigma^{\prime \prime}$ measured at some appropriate frequency.

\subsection{Sample preparation and measurement}

For this study, sand samples were sourced from two locations in the lower North Island of New Zealand. These were then sieved into 13 different grain size fractions ranging from $\varphi=0.0$ to $\varphi=3.0$ that differ by $0.25 \varphi$ on the Krumbein scale (Krumbein \& Aberdeen, 1937). Table 6.1 shows the diameter of the sieve meshes and the resulting range of grain sizes in each sample. Hydraulic conductivity and SIP measurements were conducted on these 13 sieved fractions of sand. These 13 fractions form the series 1 samples. After the first set of measurements seven of these sieved fractions, from $\varphi=$ 0.0 to $\varphi=3.0$ that differ by $0.50 \varphi$ were sieved once again to check for consistancy. As the sands were sieved using a sieve shaker it is possible that the first series of samples may had slightly bigger or smaller grains sticking to them. When sieved for the second time it was found that there indeed was a small amount of smaller grains in the series 1 samples. These re-sieved sands of seven fractions, $\varphi=0.00,0.50,1.00,1.50,2.00,2.50$ and 3.00 , form the series 2 samples.

The samples of both series 1 and series 2 were prepared by the same procedure as explained in chapter 4 . Tight packing of all the samples ensures that there is no significant variation in hydraulic conductivity due to settling or compaction during the flow of water through the sample. Series 1 samples were saturated with $\mathrm{NaCl}$ solution of electrical conductivity $100 \mu \mathrm{Scm}^{-1}$. The solution is prepared by adding table salt to 
distilled water. After the determination of hydraulic conductivity by the constant head method the DC electrical resistivity is measured. Using the DC resistivity value and the electrical conductivity of the pore water the apparent formation factor is calculated. As explained in chapter 4 the formation factor measured at an electrical conductivity of pore water of around $100 \mu \mathrm{Scm}^{-1}$ could be an underestimate of the actual or intrinsic formation factor. Hence a correction was applied to calculate the actual formation factor as explained in section 4.9.2. The procedure for the second series of measurements differed in that the formation factor was calculated using DC measurements with fluid of three separate conductivities $\left(50 \mu \mathrm{Scm}^{-1}, 200 \mu \mathrm{Scm}^{-1}\right.$ and $\left.400 \mu \mathrm{Scm}^{-1}\right)$ and then applying the method explained in section 4.9.1. A summary of the measurements of $\varphi, F$ and $m$ is given in Table 6.1. The known mass and volume of the sample holder and sand, both when dry and when saturated, were used to calculate the porosity of the sample.

\begin{tabular}{|c|c|c|c|c|c|c|c|c|c|c|}
\hline \multicolumn{7}{|c|}{ Series 1} & \multicolumn{4}{|c|}{ Series 2} \\
\hline$\varphi$ & $\phi$ & $F$ & $M$ & $\begin{array}{c}k^{*} 10^{-10} \\
(\mathrm{~m} 2)\end{array}$ & $F_{c o r r}$ & $M_{\text {corr }}$ & $\phi$ & $F$ & $M$ & $\begin{array}{c}k^{*} 10^{-10} \\
(\mathrm{~m} 2)\end{array}$ \\
\hline 0 & 0.409 & 3.11 & 1.27 & 3.64 & 3.86 & 1.51 & 0.419 & 4.22 & 1.59 & 1.78 \\
\hline 0.25 & 0.386 & 2.97 & 1.14 & 2.15 & 3.7 & 1.37 & & & & \\
\hline 0.5 & 0.389 & 3.09 & 1.2 & 1.29 & 3.85 & 1.43 & 0.374 & 4.48 & 1.51 & 1.56 \\
\hline 0.75 & 0.404 & 2.9 & 1.18 & 1.09 & 3.61 & 1.42 & & & & \\
\hline 1 & 0.394 & 3 & 1.18 & 0.8 & 3.73 & 1.41 & 0.415 & 3.47 & 1.41 & 1.02 \\
\hline 1.25 & 0.433 & 2.75 & 1.21 & 1.06 & 3.42 & 1.47 & & & & \\
\hline 1.5 & 0.401 & 3.11 & 1.24 & 0.527 & 3.87 & 1.48 & 0.452 & 3.58 & 1.61 & 0.447 \\
\hline 1.75 & 0.44 & 3.05 & 1.36 & 0.335 & 3.79 & 1.63 & & & & \\
\hline 2 & 0.43 & 3.13 & 1.35 & 0.273 & 3.89 & 1.61 & 0.452 & 3.8 & 1.68 & 0.315 \\
\hline 2.25 & 0.424 & 3.07 & 1.31 & 0.209 & 3.82 & 1.56 & & & & \\
\hline 2.5 & 0.415 & 3.1 & 1.29 & 0.147 & 3.86 & 1.54 & 0.452 & 3.86 & 1.7 & 0.194 \\
\hline 2.75 & 0.424 & 3.16 & 1.34 & 0.171 & 3.93 & 1.59 & & & & \\
\hline 3 & 0.419 & 3.09 & 1.3 & 0.0979 & 3.85 & 1.55 & 0.452 & 3.83 & 1.69 & 0.0811 \\
\hline
\end{tabular}

Table 6.1: Porosity $(\phi)$, formation factor $(F)$ and cementation exponent $(M)$ for Series 1 and Series 2 samples, and measured values of $(k)$. For the first series of measurements corrected values of $F$ and $m$ are discussed in the text and are also listed. The uncertainties are $8 \%, 3 \%, 9 \%$ and $7 \%$ respectively for porosity, formation factor, cementation exponent and measured permeability. Uncertainty for corrected formation factor and cementation exponents are $6 \%$ and $9 \%$ respectively. 


\subsection{SIP and hydraulic conductivity measurements on sieved sands}

The measurements on each sample start with recording the dry weight of the sample for porosity calculations. Then the hydraulic conductivity is measured while water of known electrical conductivity is flowing through the sample. Once the hydraulic conductivity measurements are completed the DC resistivity is recorded at that particular pore water conductivity. For Series 1 samples DC resistivity was recorded only at one pore water conductivity, while it was recorded for three different values for the Series 2 samples. After this the sample holder was made tight at both ends and moved to a chamber made up of thermally insulating material for keeping the temperature stable. The temperature fluctuations in the chamber were of the order of $\pm 1^{\circ} \mathrm{C}$. The sample was left for about 48 hours then repeated SIP measurements were conducted.

Figure 6.5 (a) and (b) show the recorded SIP phase spectra of Series 1 and Series 2 samples respectively. All of the measured a samples exhibit a clear peak in measured phase. The position of this peak is spread between 0.001 and $1 \mathrm{~Hz}$. The position of the peak keeps moving towards the higher frequencies as the grain size decreases.

The measured spectra are then fitted to the Cole-Cole model as given by equation (6.5) using the data analysis routine Solver in MS Excel. The fitting was done between the frequencies $0.001 \mathrm{~Hz}$ to $1 \mathrm{~Hz}$ as the response above $1 \mathrm{~Hz}$ is suspected to be due to instrumentation effects (Breede et al., 2012) and possibly because of Maxwell-Wagner Polarization (Chelidze and Guéguen, 1999). The fit to the phase response has been refined using the curve fitting package in MATLAB which yields $93 \%$ confidence limits on the recovered parameters. Values of the fit parameter $R^{2}$ for the phase data is in all cases in excess of 0.975 . Figure 6.6 shows an example of the quality of fit. The Cole-Cole parameters estimated from the Cole-Cole modelling are listed in Table 6.2.

It can be seen from Table 6.2 that there is a notable difference between the estimated values of $\tau_{C C}$ for samples with the same $\varphi$ values. The $\tau_{C C}$ values for the Series 2 samples are larger than the respective values of Series 1 by a factor of 1.5 to 3 . This is discussed later in section 6.7. To remove dependence on the nature of the fluid, the measurements of hydraulic conductivity $(K)$ made on each sample have been converted to values of permeability ( $k$ ) using equation (4.3). The values of $k$ are listed in Table 6.1. 
Uncertainties in these values, derived from the repeat measurements, are typically about $7 \%$.
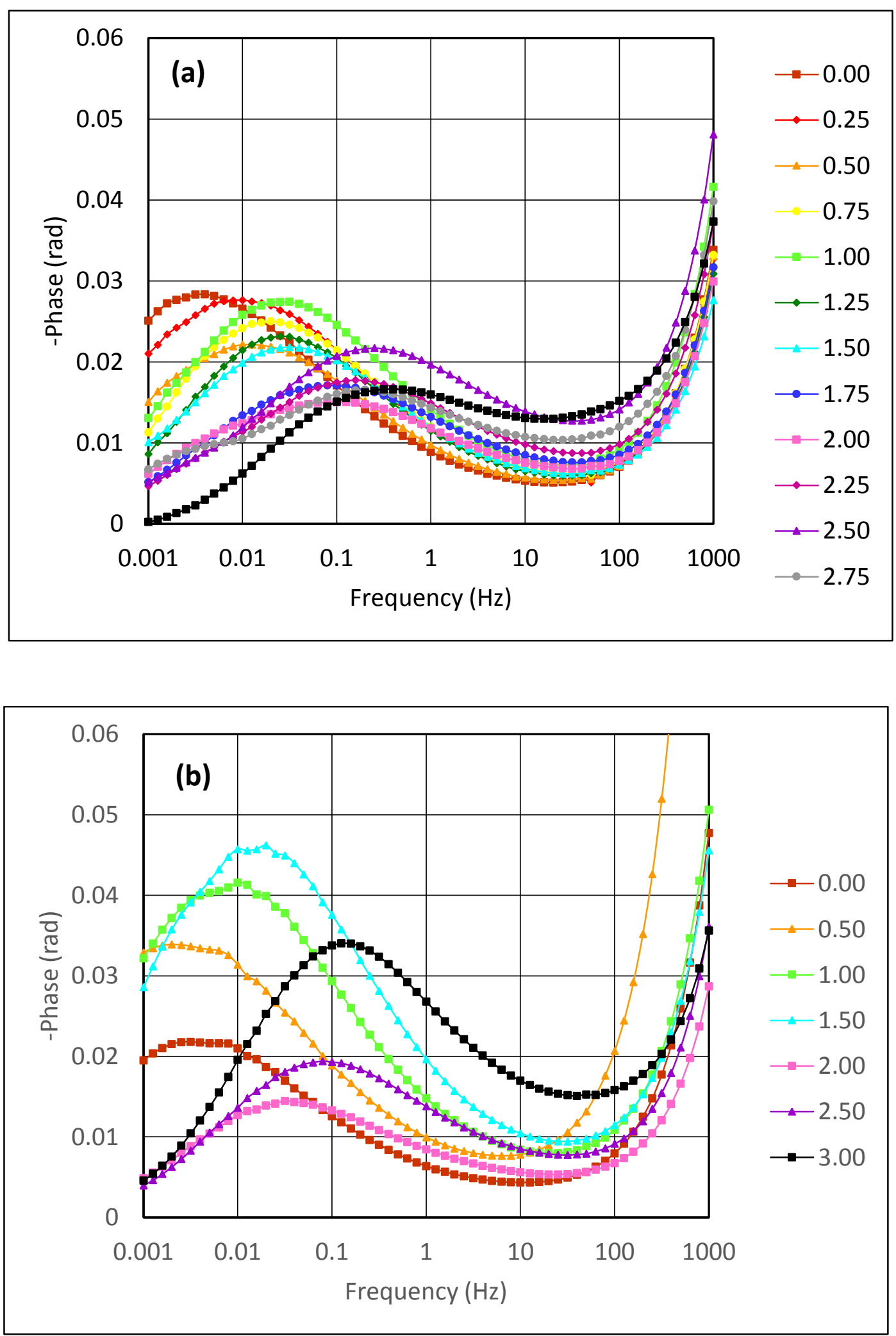

Figure 6.5: SIP spectra measured on sands of specified grain sizes (a) series 1 (b) series 2. 


\begin{tabular}{|c|c|c|c|c|}
\hline$\varphi$ & $\begin{array}{c}\sigma_{o} \\
\left(\mu \mathrm{Scm}^{-1}\right)\end{array}$ & $\tau_{\mathrm{CC}}(\mathrm{s})$ & $c$ & $M$ \\
\hline \multicolumn{5}{|c|}{ Series 1} \\
\hline 0 & 39.8 & $36.4 \pm 2.2$ & 0.414 & 0.153 \\
\hline 0.25 & 42.7 & $12.9 \pm 0.2$ & 0.424 & 0.148 \\
\hline 0.5 & 47.3 & $10.3 \pm 0.3$ & 0.439 & 0.115 \\
\hline 0.75 & 46.4 & $5.60 \pm 0.36$ & 0.493 & 0.116 \\
\hline 1 & 37.9 & $4.60 \pm 0.14$ & 0.482 & 0.129 \\
\hline 1.25 & 49.8 & $4.37 \pm 0.26$ & 0.523 & 0.101 \\
\hline 1.5 & 54.9 & $3.86 \pm 0.11$ & 0.481 & 0.104 \\
\hline 1.75 & 50.3 & $1.50 \pm 0.11$ & 0.465 & 0.087 \\
\hline 2 & 49.5 & $1.60 \pm 0.11$ & 0.401 & 0.089 \\
\hline 2.25 & 43.6 & $0.863 \pm 0.033$ & 0.46 & 0.09 \\
\hline 2.5 & 31.7 & $0.549 \pm 0.021$ & 0.467 & 0.107 \\
\hline 2.75 & 36.9 & $0.525 \pm 0.152$ & 0.356 & 0.106 \\
\hline 3 & 43.5 & $0.508 \pm 0.088$ & 0.605 & 0.065 \\
\hline \multicolumn{5}{|c|}{ Series 2} \\
\hline 0 & 42.7 & $34.5 \pm 2.5$ & 0.444 & 0.114 \\
\hline 0.5 & 36.6 & $51.6 \pm 3.4$ & 0.406 & 0.187 \\
\hline 1 & 45.1 & $15.1 \pm 0.4$ & 0.448 & 0.202 \\
\hline 1.5 & 47.6 & $7.44 \pm 0.13$ & 0.465 & 0.214 \\
\hline 2 & 70.6 & $3.26 \pm 0.22$ & 0.508 & 0.066 \\
\hline 2.5 & 60.1 & $1.52 \pm 0.11$ & 0.536 & 0.084 \\
\hline 3 & 51.2 & $0.931 \pm 0.072$ & 0.564 & 0.137 \\
\hline
\end{tabular}

Table 6.2: Results of Cole-Cole fitting to SIP amplitude and phase spectra between 0.001 and $1 \mathrm{~Hz}$ for Series 1 and Series 2 samples. The uncertainty in estimated Cole-Cole parameters is $\pm 7 \%$.

\subsection{Correction to the formation factor for Series 1 samples.}

Using the values of formation factor $F$, and cementation exponent $m$ listed in Table 6.1, and taking the grain size $d$ as the size of the respective sieves in which they are collected during the sieving, the permeability of both Series 1 and Series 2 can be calculated using equation (6.13). The comparison between the predicted permeability given by (6.13) and the measured value is shown in Figure 6.7(a). The large uncertainties in the 

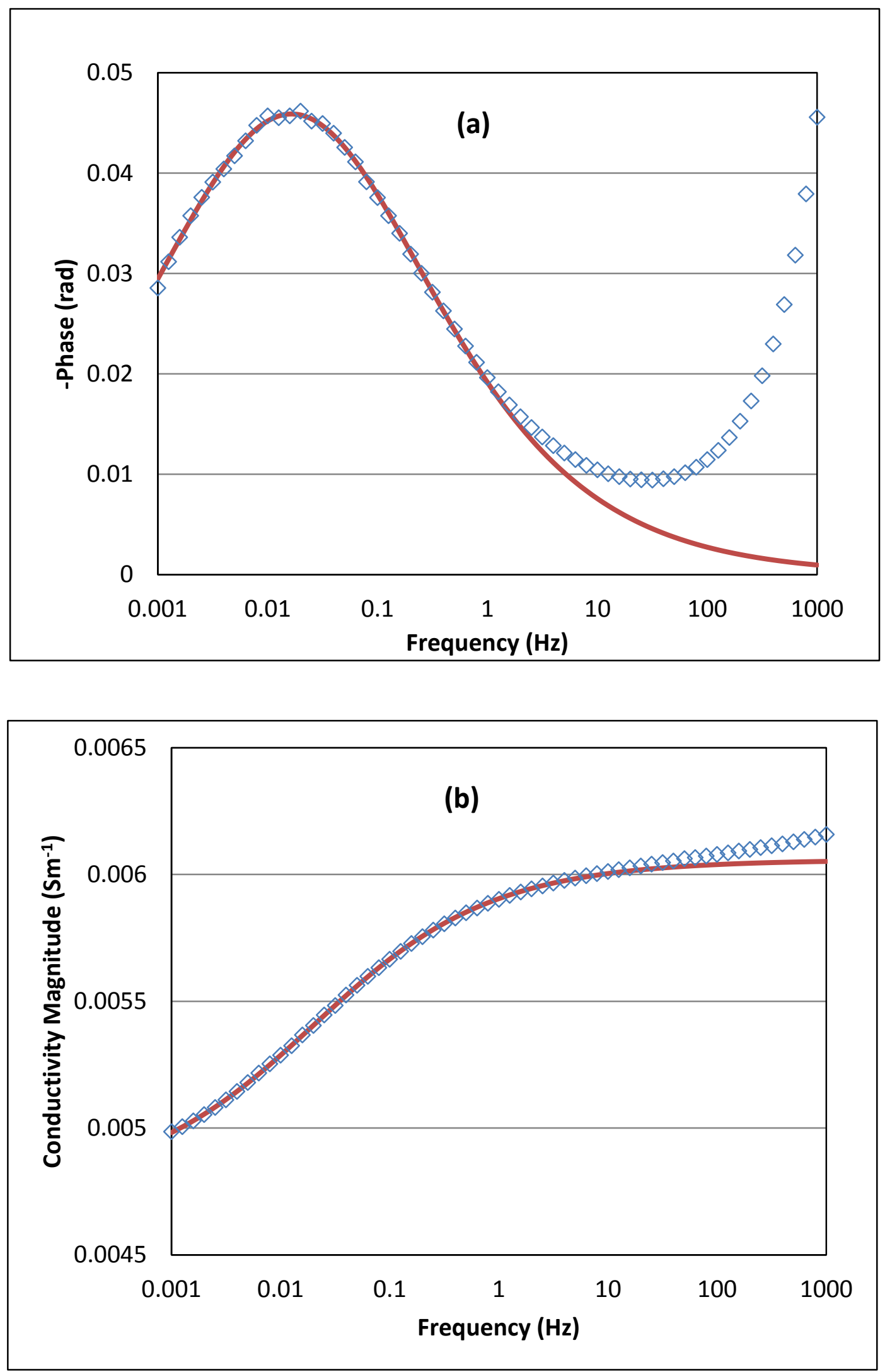

Figure 6.6: Fit of a Cole-Cole model to SIP amplitude and phase for $\varphi=1.5$. Fitting is in the range of $0.001-1 \mathrm{~Hz}$. (a) phase, (b) conductivity magnitude 
Predicted permeability arise the appearance of the term $F(F-1)^{2}$ in the denominator. While the calculated permeabilities for both Series 1 and Series 2 are close to a 1:1 line they are offset from each other by an amount significantly more than the uncertainties involved in both permeability and formation factor. Again a look at Table 6.1 shows that even though the porosities are nearly the same for respective samples of both series, the formation factor of Series 1 is lower than that of Series 2. This points to the fact that the formation factor measured at around $100 \mu \mathrm{Scm}^{-1}$ is an underestimate and hence require a correction. As the sand, except for removal of a tiny fraction of smaller size grains and the packing method involved are identical for both Series 1 and Series 2 a correction factor can be calculated based on formation factor for Series 2 samples. The apparent formation factor given by equation (4.14) is

$F_{a}=\frac{F \sigma_{w}}{\sigma_{s} F+\sigma_{w}}$

where $\sigma_{s}$ is the term indicating the contribution from surface conductivity. This surface conductivity term can be determined from a plot like the one shown in Figure 4.26 and then the apparent formation factor $F_{a}$ at any value of the pore water conductivity $\sigma_{w}$ can be calculated. When such an analysis was done on the seven samples of Series 2, it was found that the apparent resistivity measured at $100 \mu \mathrm{Scm}^{-1}$ is about $80 \pm 6 \%$ of the intrinsic or true value of the formation factor. The corrected formation factor $\left(F_{c o r r}\right)$, and the corresponding corrected cementation exponent $\left(m_{\text {corr }}\right)$, are listed in Table 6.1. A plot of measured permeability against permeability calculated using equation (6.13) with the corrected formation and cementation exponent are shown in Figure 6.7(b). As expected now equation (6.13) makes better prediction of permeability.

\subsection{Relationship between $\tau_{C C}$ and grain size}

Equation (6.14) indicates that $\tau_{C C}$ is proportional to the square of the grain diameter. A plot of the recovered values of $\tau_{C C}$ against the sieve mesh size is shown in Figure 6.8. Power law relationships provide excellent fits to the data from both series of measurements. The exponents obtained in the power law fit are, $1.97 \pm 0.26$ and $2.00 \pm$ 0.51 , respectively for the two data series. Both of these encompass the power of 2 predicted by equation (6.14). Calculated values for the diffusion coefficient are, respectively, $7.1 \times 10^{-9}$ and $2.2 \times 10^{-9} \mathrm{~m}^{2} \mathrm{~s}^{-1}$. These are both larger than values generally 

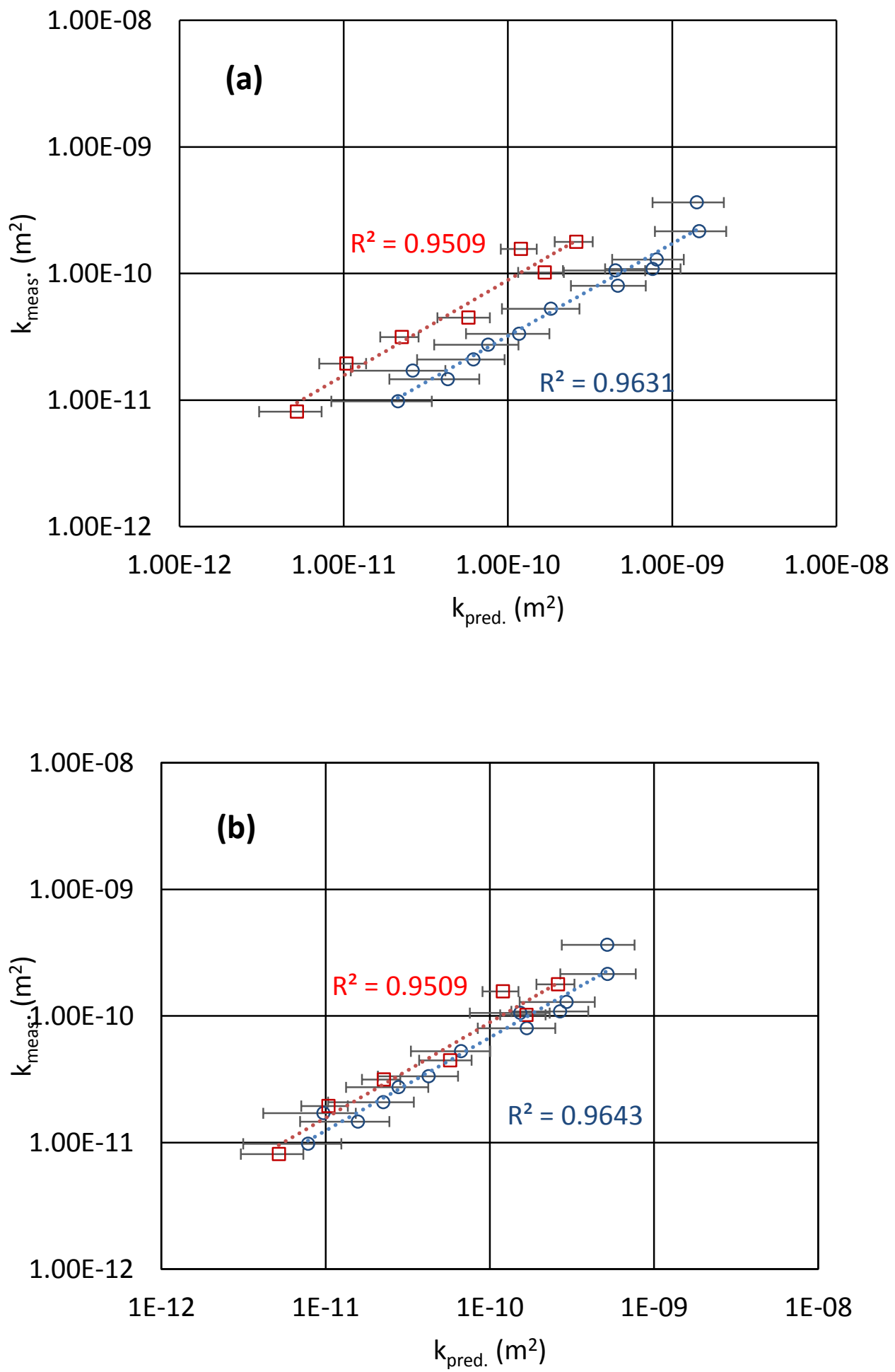

Figure 6.7: (a) Measured permeability plotted against predicted permeability given by equation (6.13) Series 1 open circles, Series 2 - squares. (b) as (a) after correction of the formation factor for data series 1 . Uncertainties in the predicted values for Series 2 data come from the fit of equation (54) to apparent formation factor data. Uncertainties in the corrected values for series 1 data come from the estimate of uncertainty in the degree to which the intrinsic formation factor is underestimated by a single determination at one fluid conductivity. 
quoted for $\mathrm{Na}^{+}$in clean sands (e.g. $1.3 \times 10^{-9} \mathrm{~m}^{2} \mathrm{~s}^{-1}$ at $25^{\circ} \mathrm{C}$, Revil et al., 2012) but have large uncertainties associated with them, which again encompass the expected value. The experimental data could therefore be considered to confirm the expected relationship between $\tau_{c c}$ and $d$. However, even though both series display an excellent power law relationship, there is a disparity in the recovered values of the $\tau_{c c}$. There are two possible reasons for this. The first is that the Series 1 samples had more of the smaller size grains sticking to them which may have had the effect of reducing the effective pore size which in turn predicts a decrease in the value of $\tau_{c c}$. The second reason may be the difference in electrical conductivity of the pore fluid as explained in section 6.10. For the second series, DC resistivity measurements were done at three different pore water conductivities starting with $400 \mu \mathrm{Scm}^{-1}$ and coming down to 50 $\mu \mathrm{Scm}^{-1}$. But for the first series the DC resistivity measurements were made only at 100 $\mu \mathrm{Scm}^{-1}$. Both series of samples were left for at least 48 hours before making SIP measurements. It has been observed for all samples that after they are saturated with pore water of a particular electrical conductivity there was a slow and steady increase in the pore fluid conductivity. Hence the pore water conductivity at which the Series 2 samples were stabilised could have been slightly less than that of the Series 1 samples and this might have contributed to a small increase in $\tau_{c c}$ of the Series 2 samples.

\subsection{SIP and hydraulic conductivity measurements on mixtures of grain sizes}

The observation of sensitivity of $\tau_{c c}$ to grain size indicate that it is worth investigating the effect of the presence of smaller grains along with larger grains. This is very important because in a natural setting the geological materials consist of a grain size distribution rather than a single grain size. An investigation was done with samples prepared by mixing grains of size $\varphi=0.25$ and $\varphi=2.50$. Table 6.3 shows the different proportions of the two grain sizes in each sample, the measured porosity, formation factor, cementation exponent and permeability, and also the recovered value of $\tau_{c c}$ determined from Cole-Cole modelling of the SIP responses. The SIP phase spectra measured for these samples are shown in Figure 6.9.

The two grain sizes chosen are such that the smaller grains can potentially fit in the void formed due to the larger grains. For this to occur the smaller grain diameter should be 


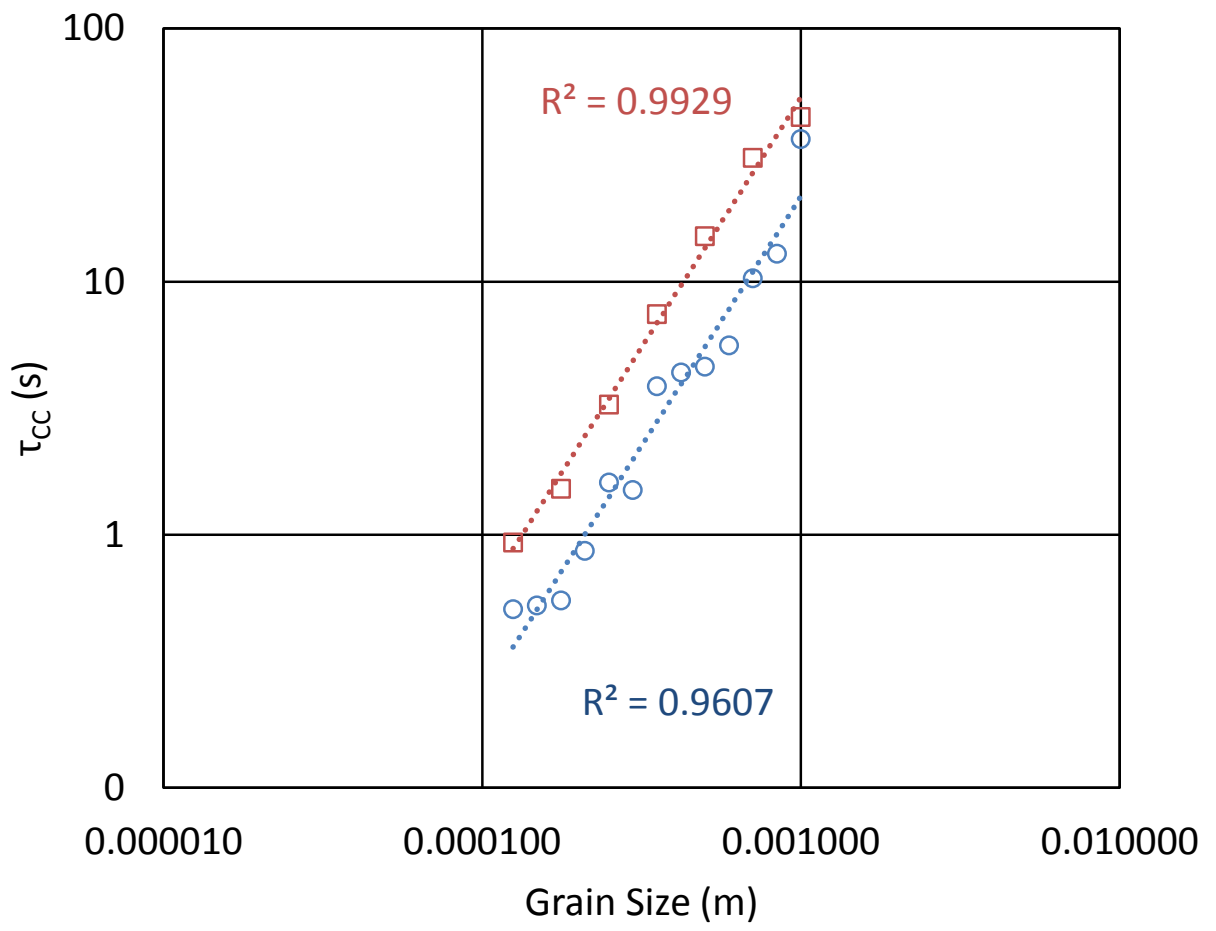

Figure 6.8: Relaxation time derived from Cole-Cole modelling plotted against the grain size. Series 1 - open circles, Series 2 -squares. Uncertainties in the relaxation time are generally too small to show.

at most 0.29 times smaller than the size of the larger grain (Zong, 2002). The range of ratio of diameter of the grains used for making the mixtures is in the range of 0.177 to 0.25. If spherical grains of uniform diameter are considered the smallest porosity that can be achieved by close-packing is approximately $26 \%$ (Hales, 2005). For loose random packing, such as achieved by the method used to fill the sample holder, the expected porosity is 40\% (Scott, 1960; Scott \& Kilgour, 1969). As can be seen from Table 6.3 and Figure 6.10(a) this corresponds closely to the measured porosity for both the samples consisting of a single grain size. As the smaller grains fill up the voids the porosity of the mixture decreases with increasing percentage of smaller grains. As can be seen from both Table 6.3 and Figure 6.10(a) the porosity reaches a minimum and then increases again to the expected $40 \%$, showing a variation that can be extremely well represented by a quadratic function. A variation in formation factor is also is expected with variation in the percentage of the smaller grain size. As more and more voids are filled the net resistance of the sample increases. This is because the resistance of the sand grains is much larger than the resistance of pore water. Figure 6.10(a) shows that the formation factor increases and then decreases as expected. 
The changes in both permeability and the calculated Cole-Cole relaxation time with increasing percentage of the smaller diameter grains are shown in Figure 6.10(b). The permeability keeps decreasing with the increasing percentage of smaller grain sizes. This is expected as the percentage of smaller grain increases more and more pore space is occupied by the smaller grain, and this reduces the space available for flow of water through the medium. The reduction in permeability observed is close to 1 order of magnitude. The relaxation time $\tau_{C C}$ behaves in a similar fashion, with even the addition of $10 \%$ of the smaller diameter grains leading to a nearly $50 \%$ decrease in $\tau_{c c}$

Such sensitivity of $\tau_{c c}$ to the presence of smaller grain support the hypothesis that the mismatch of the lines in Figure 6.8 could be a result of the presence of smaller grains. This suggests that there could be an "effective grain diameter" $\left(d_{e}\right)$ when it comes to mixtures of sand. For Series 1 samples the sieve mesh size multiplied by some constant reduction factor $C$ would bring consistency between the sets of measurements. It is found that a value of $C \approx 0.625$ brings the Series 1 data into best alignment with the Series 2 data for which $C=1$ is assumed. It can be calculated that the relaxation times have been reduced by about $40 \%$. It cannot be considered that the mismatch in Figure 6.8 is caused by the presence of smaller grains alone. There could be another factors like variations in pore fluid conductivity as discussed in section 6.7 and 6.10.

\subsection{Prediction of permeability from SIP for New Zealand coastal aquifers}

Equations (6.13) and (6.15) show that both the permeability and $\tau_{c c}$ are proportional to $d^{2}$. Plots of measured permeability and $\tau_{c c}$ against the grain size shown in Figure 6.1 and Figure 6.8 strongly support this. Hence given that the formation factor, porosity, and the counterion diffusion coefficient $D_{i}$ are known, equations (6.16) and (6.18) should be able to make good permeability predictions. The equations (6.16) and (6.18) differ in choosing the dynamic pore radius that is assumed to control the permeability as the grain diameter or pore radius respectively.

The result of plotting measured values of permeability against those predicted by these two equations is shown in Figure 6.11. In all the permeability calculations the value of $1.3 \times 10^{-9} \mathrm{~m}^{2} \mathrm{~s}^{-1}$, is used for the diffusion coefficient $\left(D_{i}\right)$. This value is suggested by Revil et al. (2012) for a pore fluid that consists of a majority of $\mathrm{Na}^{+}$ions. 


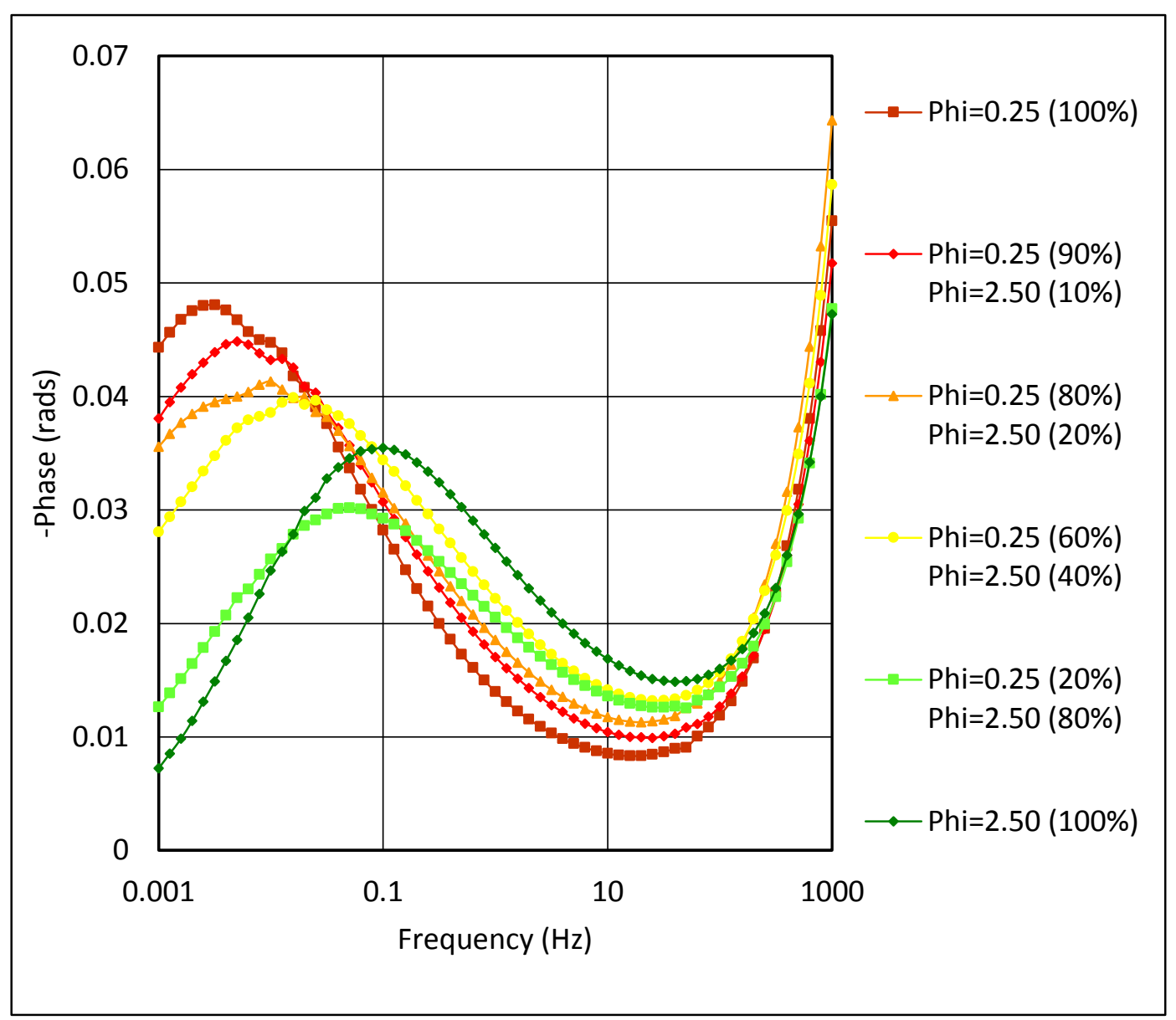

Figure 6.9: SIP phase spectra measured on mixtures of saturated sands with different proportions of grain sizes $\varphi=0.25$ and $\varphi=2.50$.

\begin{tabular}{|c|c|c|c|c|c|}
\hline $\begin{array}{c}\boldsymbol{\varphi} \boldsymbol{\varphi}=\mathbf{0 . 2 5}- \\
\boldsymbol{\%} \boldsymbol{\varphi}=\mathbf{2 . 5 0}\end{array}$ & $\boldsymbol{\phi}$ & $\boldsymbol{F}$ & $\boldsymbol{m}$ & $\begin{array}{c}\boldsymbol{k}^{*} \mathbf{1 0}^{-\mathbf{1 0}} \\
\mathbf{( m}^{\mathbf{2}}\end{array}$ & $\begin{array}{c}\boldsymbol{\tau}_{\mathbf{C C}} \\
(\mathbf{s})\end{array}$ \\
\hline $100-0$ & 0.409 & 3.83 & 1.5 & 1.39 & $36.7 \pm 1.5$ \\
\hline $90-10$ & 0.384 & 4.38 & 1.55 & 0.583 & $20.1 \pm 0.9$ \\
\hline $80-20$ & 0.371 & 4.74 & 1.57 & 0.57 & $15.4 \pm 0.5$ \\
\hline $60-40$ & 0.35 & 4.81 & 1.5 & 0.282 & $6.43 \pm 0.18$ \\
\hline $20-80$ & 0.358 & 4.66 & 1.5 & 0.098 & $2.27 \pm 0.09$ \\
\hline $0-100$ & 0.4 & 4.23 & 1.57 & 0.119 & $1.25 \pm 0.09$ \\
\hline
\end{tabular}

Table 6.3: Porosity $(\phi)$, formation factor $(F)$, cementation exponent $(m)$, permeability $(k)$ and Cole-Cole relaxation time $\left(\tau_{C C}\right)$ of samples consisting of mixtures of grain sizes $\varphi=0.25$ and $\varphi=2.50$. The uncertainties are $8 \%, 3 \%, 9 \%$ and $7 \%$ respectively for porosity, formation factor, cementation exponent and measured permeability 

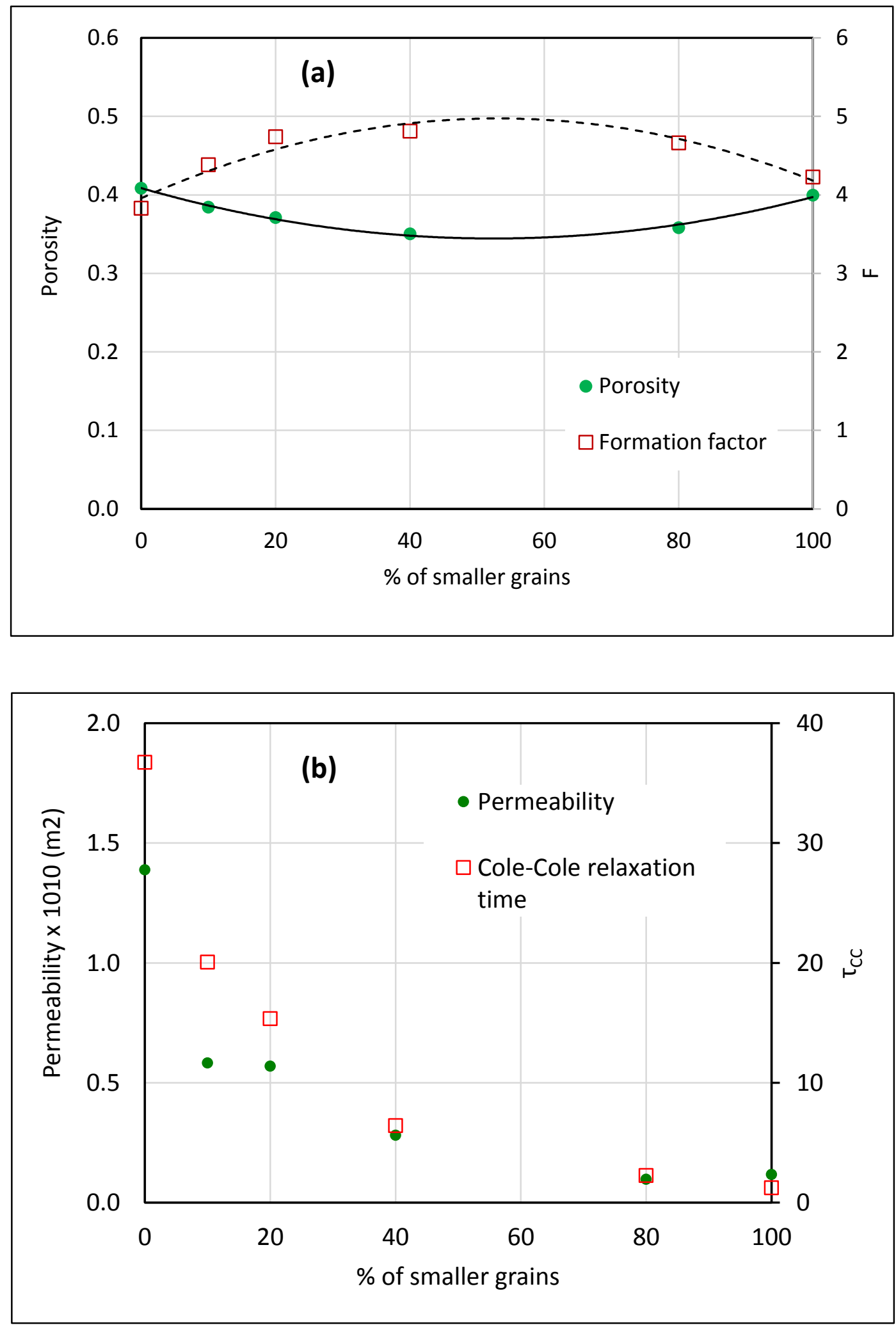

Figure 6.10: Variation in (a) measured porosity and formation factor, (b) measured permeability and Cole-Cole relaxation time, for mixtures of saturated sands with different proportions of grain sizes $\varphi=0.25$ and $\varphi=2.50$. 
Figure 6.11 shows that both equations (6.16) and (6.18) are possibly useful predictors for all the three types of samples used, i.e. Series 1, Series 2 and the mixtures. While equation (6.16) under-estimates the permeability slightly, equation (6.18) overestimates it by close to an order of magnitude. This is not surprising as a look at the denominator of equation (6.16) and (6.18) shows that the only difference between the two equation is an absence of the term $m^{2}(F-1)^{2}$. For unconsolidated sands, the average value of formation factor is around 4 and that of the cementation exponent is about 1.5. With these values it can be seen that for the same value of $\tau_{C C}$ and $D_{i}$ the predicted permeabilities differ by a factor of 20 between equations (6.16) and (6.18). A measure of the goodness of fit is given by the RMS defined as

$$
\varepsilon_{\text {rms }}=\sqrt{\frac{1}{N} \sum\left(\log _{10}\left(k_{\text {pred }}\right)-\log _{10}\left(k_{\text {meas }}\right)\right)^{2}}
$$

with values of $\varepsilon_{r m s}$ of 0.473 for equation (6.16) (Figure 6.11(a)) and 0.958 for equation (6.18) (Figure 6.11(b)).

While equation (6.16) and (6.18) make reasonable predictions compared to any other method, it is worth investigating what is it that prevents both equations from making predictions closer to the $1: 1$ line. It has to be remembered that approximations were involved in the formulation of these equations that connect the permeability to the dynamic pore radius. Given the nature of the assumptions in equation (6.16), and the plots in Figure 6.11, it is worth investigating a correction in the form of a constant of multiplication for both equations (6.16) and (6.18) as follows.

$$
k=A \frac{\tau_{C C} D_{i}}{4 m^{2} F(F-1)^{2}}
$$

and

$$
k=B \frac{\tau_{C C} D_{i}}{4 F}
$$

Fitting equations (6.21) and (6.22) to the measured data gives the value of constants as $A=2.49 \pm 0.62$ with $\varepsilon_{r m s}=0.258$ and $B=0.122 \pm 0.034$ with $\varepsilon_{r m s}=0.291$. Figure 6.12 (a) and (b) shows the result of the fit. One factor that can affect the constants $A$ and $B$ is the diffusion coefficient $D_{i}$. 
Not all samples show a significant peak in phase response as reported by Weller et al. (2015). For such samples an empirical relationship like the one given by equation (6.19) is used to relate $k$ and $\sigma^{\prime \prime}$ - the imaginary conductivity measured at a given frequency usually at $1 \mathrm{~Hz}$. Such differences in spectra may arise from the nature of the porous medium and especially the leading mechanism that contributes to the SIP response as detailed with Figure 3.8 could be responsible for the lack of a significant peak. Even though the nature of the spectra is different a look at Figure 6.5(a) and(b) and 6.9 shows that there is indeed a variation in $\sigma^{\prime \prime}$ at $1 \mathrm{~Hz}$ corresponding to variation in grain size. This variation arises from the fact that as the grain size varies the position of the phase peak also varies and since all of them have similar shapes a variation in imaginary conductivity is expected. Hence if the Cole-Cole relaxation time is plotted against the value of the imaginary conductivity at $1 \mathrm{~Hz}$ there is a degree of correlation between $\tau_{C C}$ and $\sigma^{\prime \prime}$.This correlation suggests that, even though the low frequency phase is not flat, relationships of the kind

$$
k=\frac{a}{F^{b} \sigma^{\prime c}}
$$

as suggested by Weller et al. (2015) may still be a useful empirical predictor for permeability. The values for $a, b$ and $c$ that give the best fit for this relationship (with $k$ expressed in $\mathrm{m}^{2}$ and $\sigma^{\prime \prime}$ in $\mathrm{mSm}^{-1}$ ) are poorly constrained but yield (Figure 6.13(a)) $\log _{10} a$ $=-11.7 \pm 2.4, b=1.93 \pm 0.37$ and $c=2.21 \pm 0.91$. The misfit for this, defined as in (6.20), is $\varepsilon_{r m s}=0.315$. For samples measured at the same pore fluid conductivity then the formation factor may be replaced by dependence on the bulk DC conductivity $\left(\sigma_{0}\right)$, as outlined in Weller et al. (2015).,

$$
k=\frac{d \sigma_{o}^{b}}{\sigma^{\prime c}}
$$

where the constant $d$ is equal to $a / \sigma_{f}^{b}$. Since the SIP measurements on Series 2 and mixtures were conducted at the same fluid conductivity $50 \mu \mathrm{Scm}^{-1}$, the equation (6.23) could be tested on them. Using the exponents determined by fitting equation (6.24) to the measured permeabilities the best fitting value of $d$ is estimated as $(4.07 \pm 1.06) \mathrm{x}$ $10^{-14}$, compared to a value of $a / \sigma_{f}^{b}$ of about $9 \times 10^{-14}$, the difference most likely being 

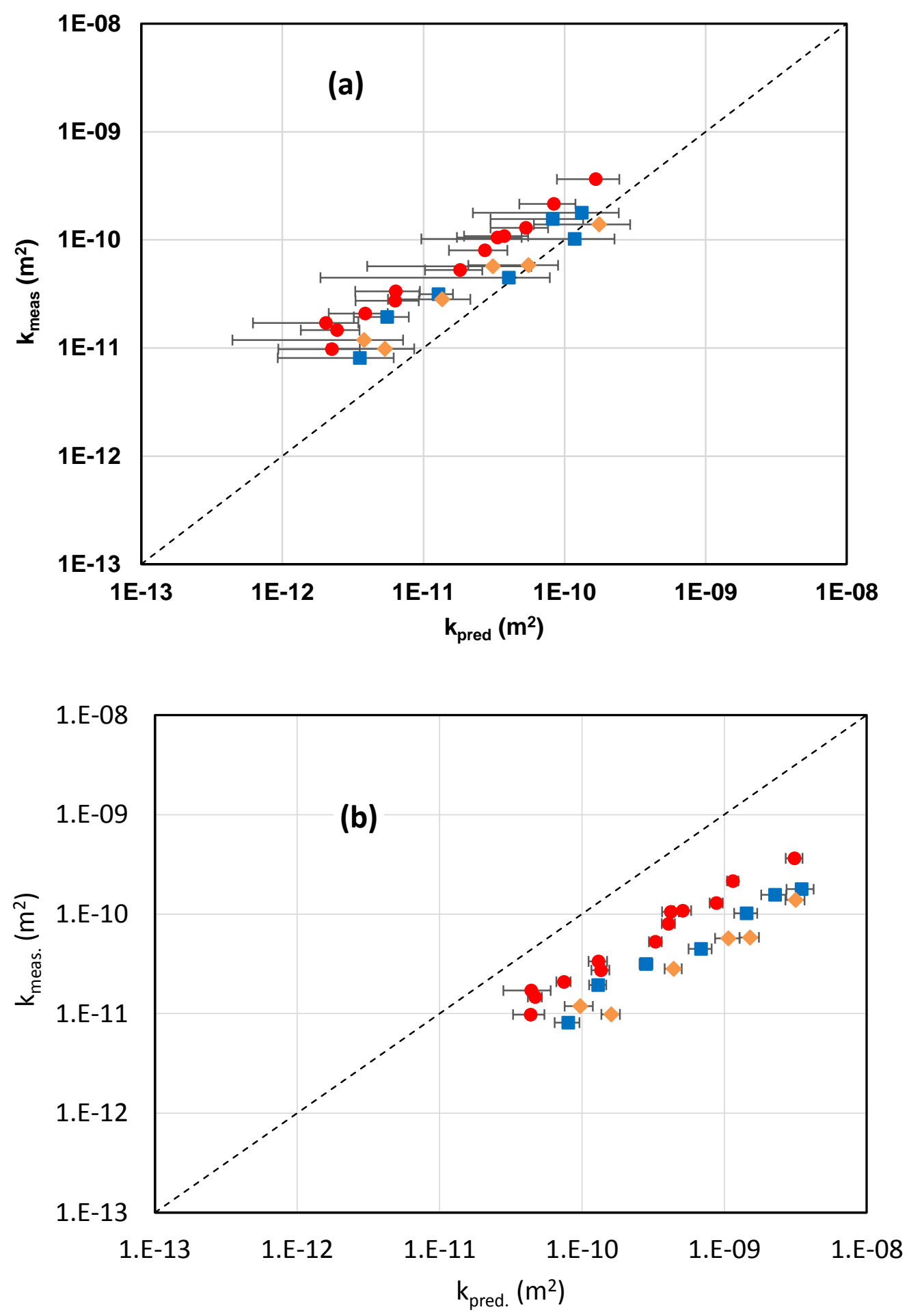

Figure 6.11: Measured permeability plotted against permeability predicted by (a) $k=\frac{\tau_{C C} D_{i}}{4 m^{2} F(F-1)^{2}}$, and (b) by $k=\frac{\tau_{C C} D_{i}}{4 F}$, Series $1-$ circles, Series $2-$ squares Mixtures - diamonds. The dashed lines show the 1:1 correspondence between measured and predicted permeability. Uncertainties are those based on uncertainties in $\tau_{C C}, F$ and $m$. 

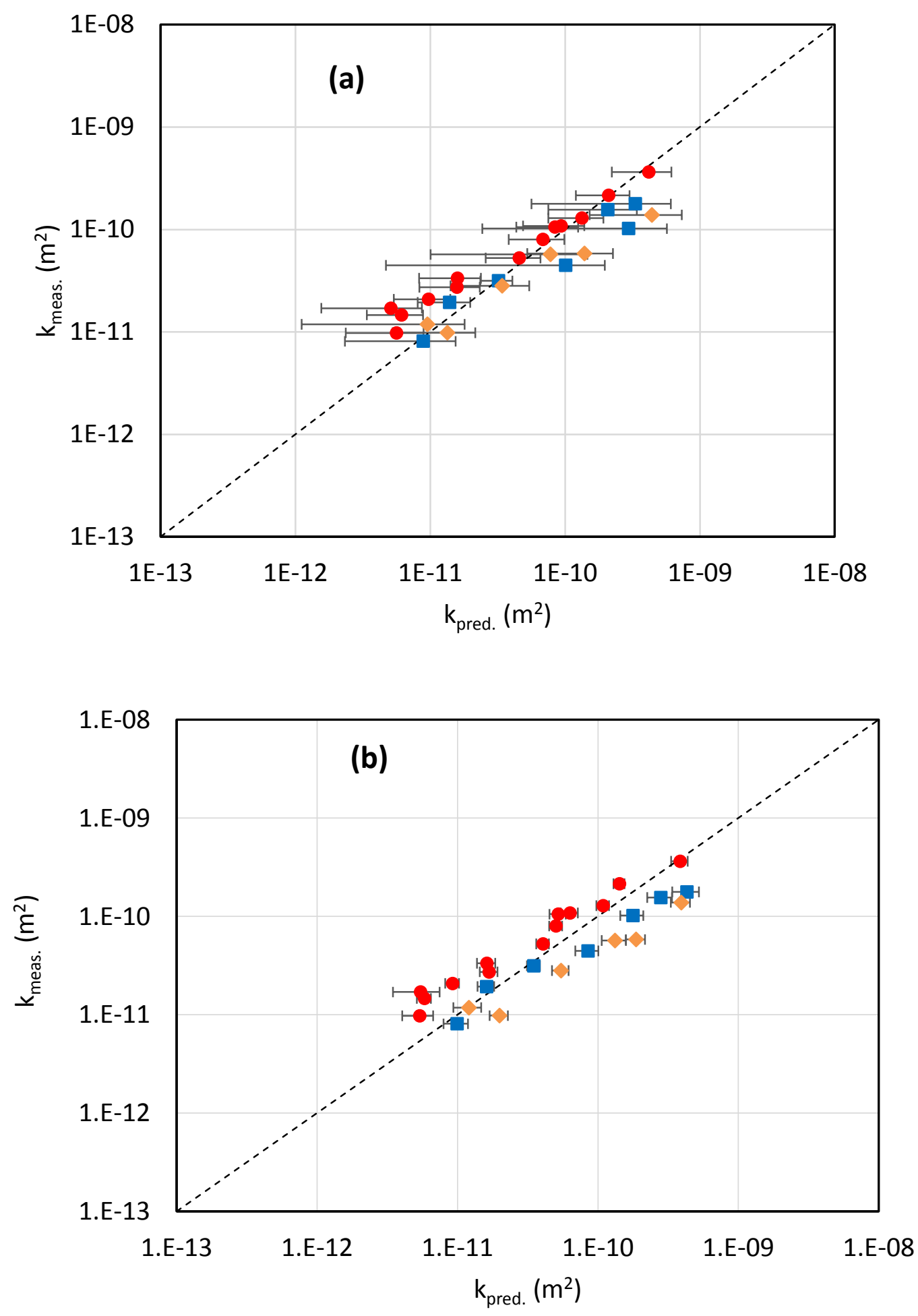

Figure 6.12: Measured permeability plotted against permeability predicted (a) by $k=A \frac{\tau_{C C} D_{i}}{4 m^{2} F(F-1)^{2}}$, and (b) by $k=B \frac{\tau_{C C} D_{i}}{4 F}$. Series $1-$ circles, Series $2-$ squares, Mixtures - diamonds. The dashed line shows the 1:1 correspondence between measured and predicted permeability. Uncertainties are those based on the uncertainties in $\tau_{C C}, F$ and $m$. 
attributable to the use of Series 1 data in the determination of $a$. The result of this fit is shown in Figure 6.13(b) and gives a misfit of $\varepsilon_{r m s}=0.195$. At first sight therefore (6.24) actually appears to be the best predictor of permeability. However, there are some significant riders to this as discussed below.

It can be considered that equations (6.21)-(6.24) are suitable for predicting the permeability of unconsolidated sands that are typical of New Zealand's shallow coastal aquifers. However, while measurements on consolidated materials like rock and sandstones are relatively abundant there are few published works related to SIP measurements on unconsolidated sediments. Revil et al. (2012) compared both equations (6.16) and (6.18) against data from samples measured by Koch et al. (2012) who made measurements on both compacted and uncompacted samples. Both the method of preparation for the compacted samples and the mean grain sizes (between 180 and $860 \mu \mathrm{m}$ ) were similar to the present study. Figure 6.14 (a) and (b) shows a test of equation (6.16) and (6.18) using data from present study and that reported for the compacted sample by Koch et al. (2012.

Figure 6.15 shows a comparison of relaxation time of data reported by Koch et al. (2012) and the present study. Again it can be seen that the $\tau_{C C}$ values are smaller compared to the present study. As discussed earlier this could be because the samples in the study of Koch et al. (2012) were not sieved to the degree to which the present samples. Hence there may be finer grains present. This in turn can bring down the value of $\tau_{C C}$. Unsurprisingly the prediction of permeability through equation (6.16), shown in Figure 6.14(a), is worse for the data of Koch et al. (2012) than for the samples in the present study; having an RMS misfit of 1.021 compared to 0.473 . When equation (6.21) is applied with the value for A determined from the data in the present study, this misfit reduces to 0.669 . The permeability predicted by $(6.18)$, shown in Figure $6.14(b)$, gives a much better prediction for Koch et al. (2012) data. This indicates that developing a single predictive expression is not easy.

An alternative predictor, which takes into account the fact that predicted values given by (6.16) are under-estimates for low values of permeability, is the empirical relationship given by fitting a power law to the measured permeability as a function of predicted permeability: 

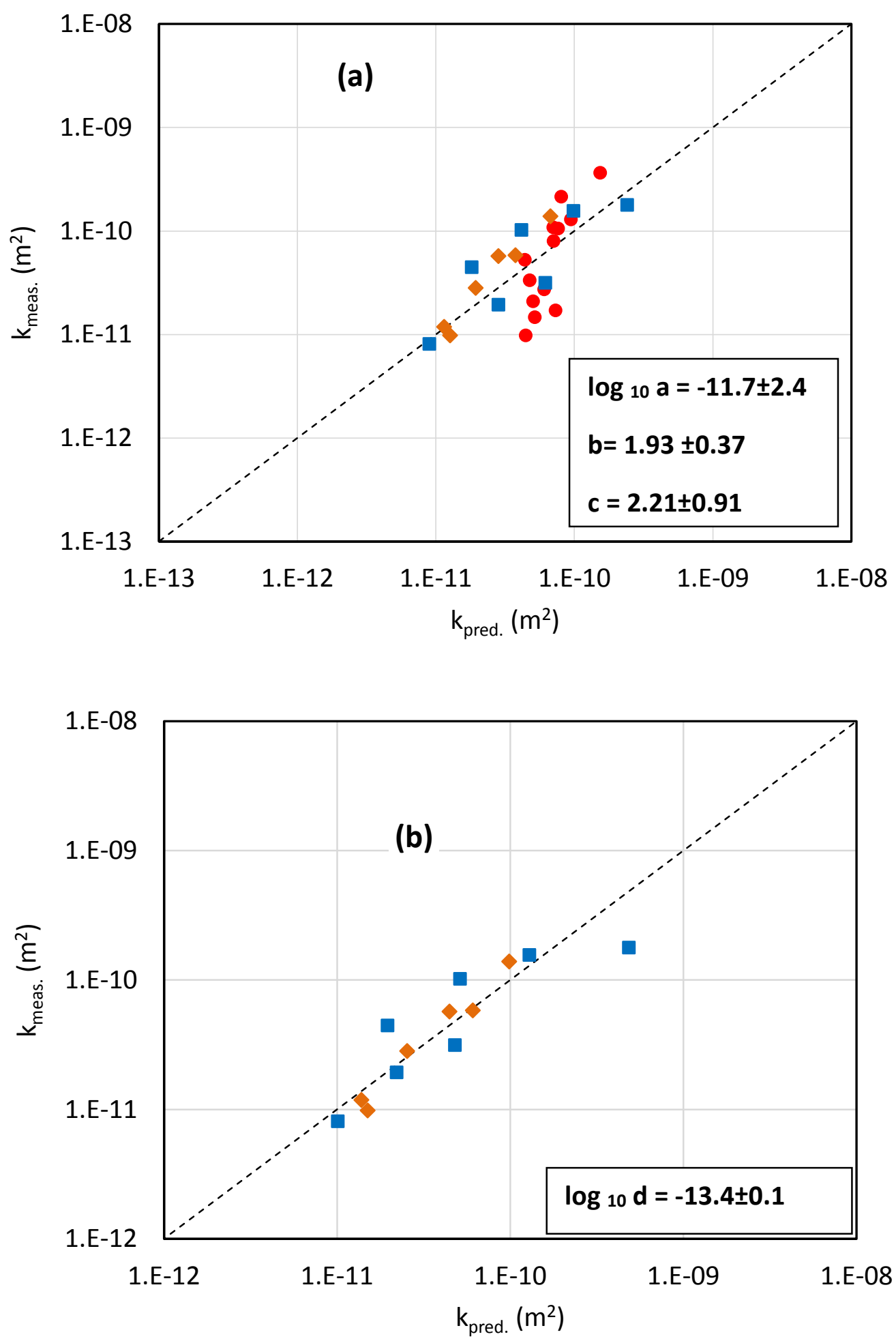

Figure 6.13: Measured permeability plotted against permeability predicted (a) by $k=\frac{a}{F^{b} \sigma^{\prime \prime c}}$ and (b) by $k=\frac{d \sigma_{o}^{b}}{\sigma^{\prime \prime c}}$. Best fitting values of $\log _{10} \mathrm{a}, \mathrm{b}, \mathrm{c}$ and $\log 10 \mathrm{~d}$ are shown. Series 1 - circles, Series 2 squares, Mixtures diamonds. The dashed line show the 1:1 correspondence between measured and predicted permeability. Uncertainities in the predicted values are not shown as these depend upon the derived values of the exponent $b$. 

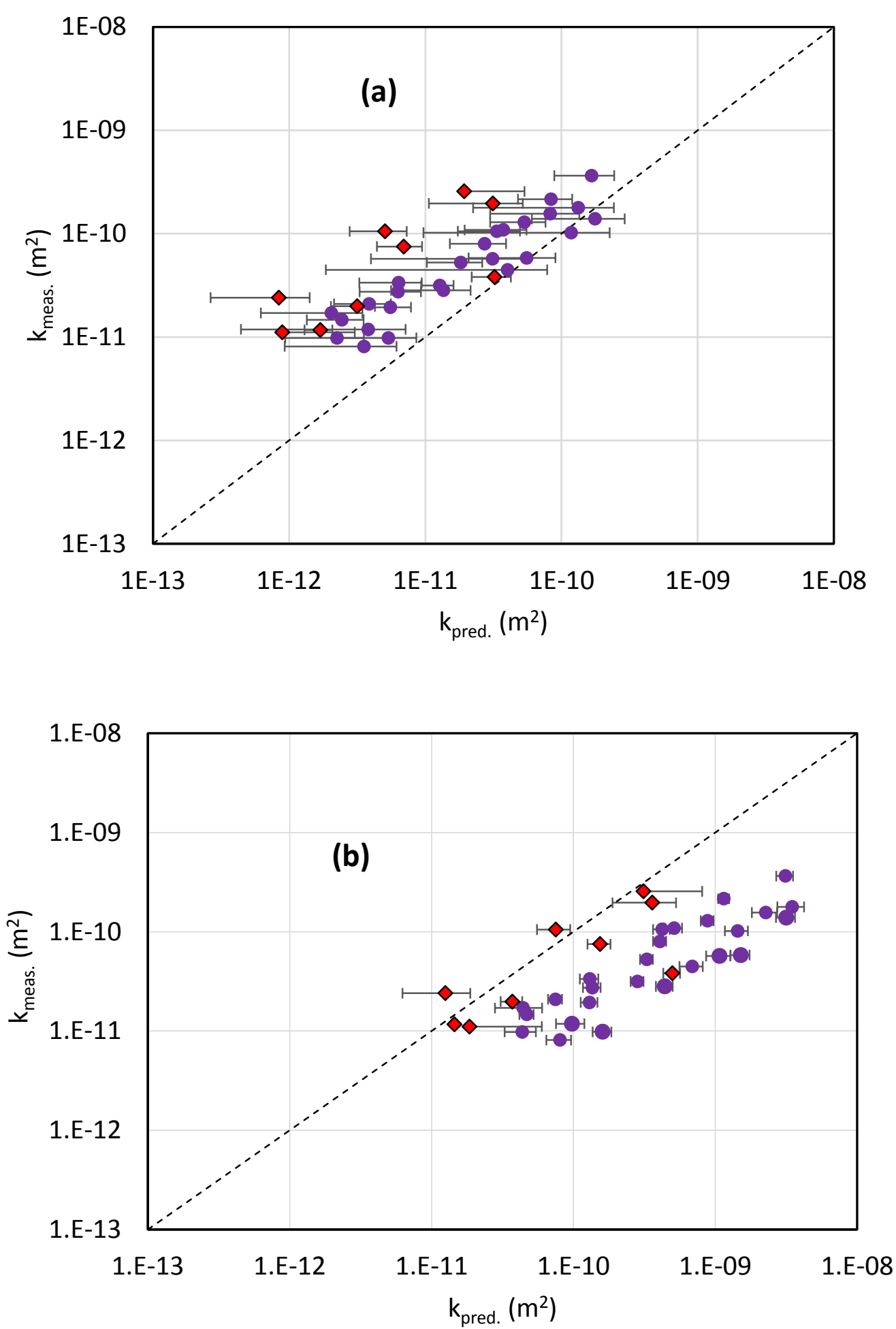

Figure 6.14: Measured permeability plotted against permeability (a) measured by $k=\frac{\tau_{C C} D_{i}}{4 m^{2} F(F-1)^{2}}$, and (b) by $k=\frac{\tau_{C C} D_{i}}{4 F}$. Circles data from this study, diamonds - data from Koch et al. (2012). The dashed lines show 1:1 correspondence between measured and predicted permeability. Uncertainties in Koch et al. (2012) data are as given by the authors. 


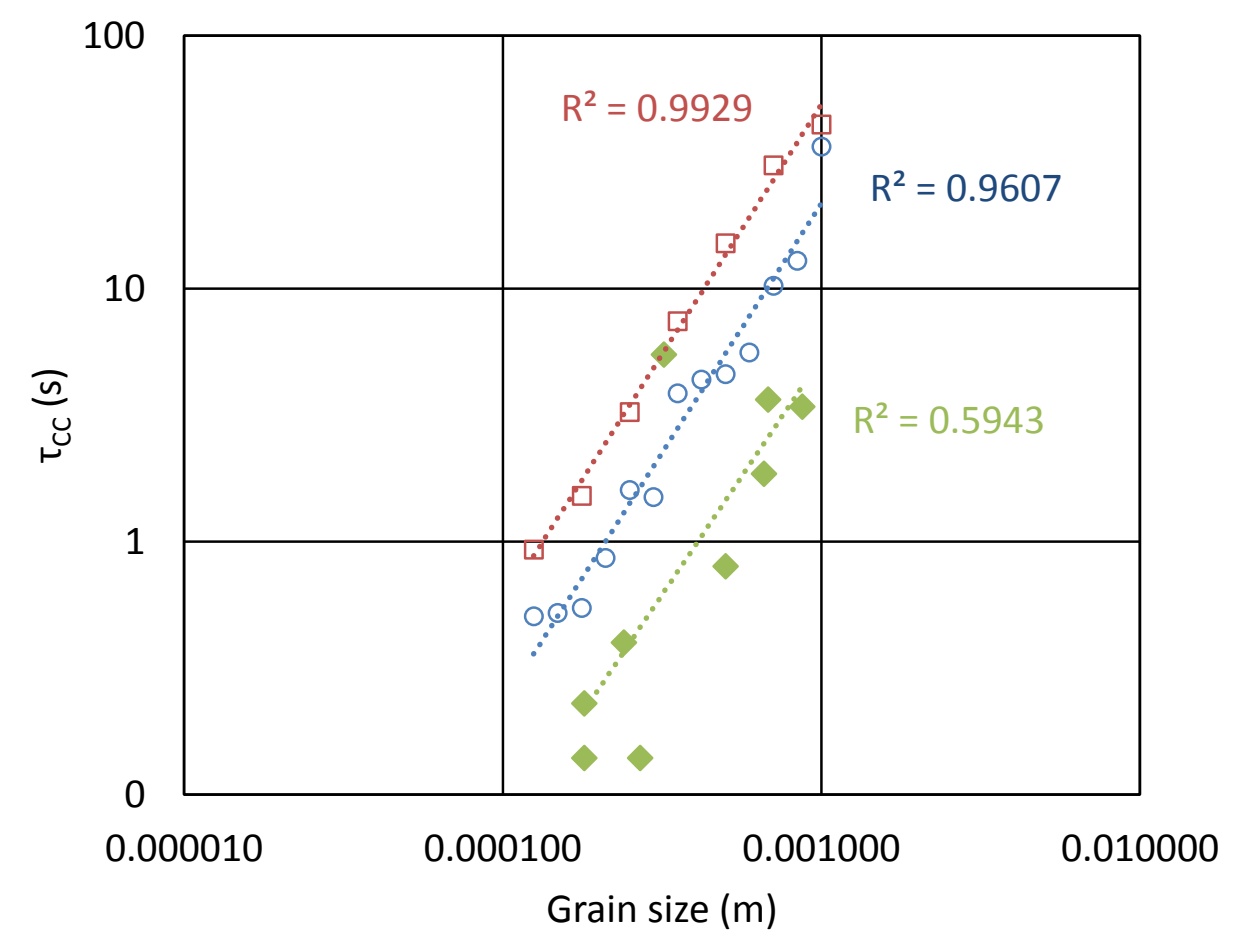

Figure 6.15: Plot of $\tau_{C C}$ against grain size. Open circles - Series 1 data from the present study; open squares Series 2 data from the present study; filled diamonds - data from Koch et al. (2012). For data from present study the sieve size is taken as the grain size. For data from Koch et al. (2012) the $d_{50}$ value determined by laser diffraction measurements is taken as the grain size.

$$
k=1.01 \times 10^{-3} k_{\text {pred }}^{0.684}
$$

where $k_{\text {pred }}$ is given by (6.16). The power law fitting was done using Microsoft Excel Solver.

Figure 6.16 shows a plot of permeability predicted using equation (6.23) with data from the present study and reported by Weller et al. (2015). In their study Weller et al. (2015) used data from a number of sources, including Weller et al. (2013), Slater et al. (2014) and $\mathrm{Xu}$ (2014), to test expressions of the kind given by equations (6.23) and (6.24). The data selected for comparison are for sands and gravels whose SIP response could be similar to that of the samples in the present study. Figure 6.16 shows that there is a good agreement between the measured permeability and the predicted permeability using $a /\left(F^{b} \sigma^{\prime c}\right)$ (with $a, b$ and $c$ as given above and in Figure 6.13(a)). The overall misfit 
is $\varepsilon_{r m s}=0.439$. Other samples used by Weller et al. (2015), including floodplain samples with low permeability and samples with clay content, were not compatible with this fit. Permeability prediction with equation (6.24) with the data reported by Weller et al. (2015) was not attempted because the measurements were conducted at different fluid conductivities.

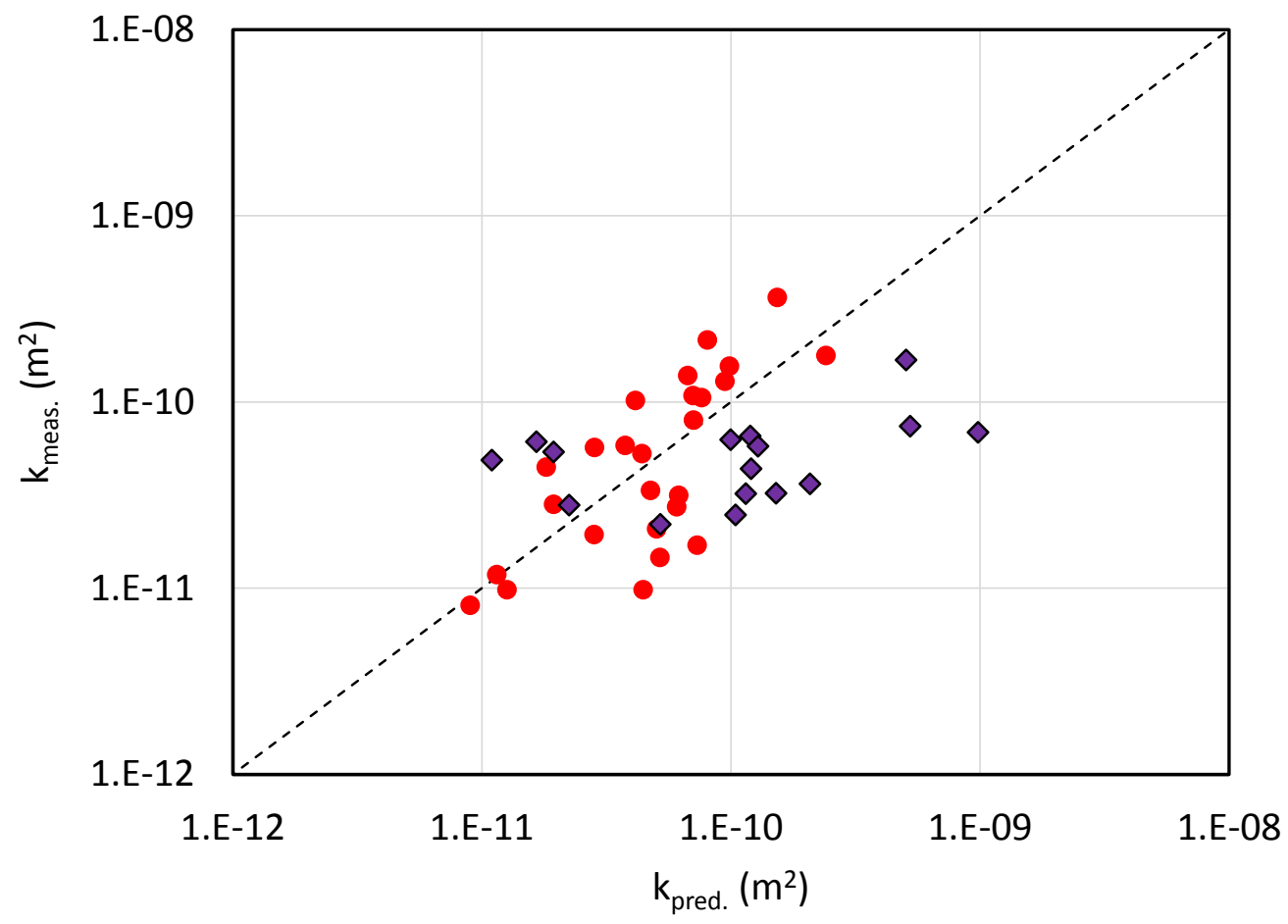

Figure 6.16. Measured permeability plotted against permeability predicted by $k=\frac{a}{F^{b} \sigma^{\prime \prime c}}$ with values of $\mathrm{a}, \mathrm{b}, \mathrm{c}$ as given in Figure 6.13(a). Filled circles data from this study; diamonds data from Weller et al. (2015). The dashed lines show 1:1 correspondence between measured and predicted permeability

While this study cannot claim to have found a single predictor for permeability, it appears that equations (6.21), (6.22) and (6.23) may all be usable predictors for permeability of New Zealand shallow sand aquifers. The constants A, B, a, b and c may be applicable only for the New Zealand aquifers.

Field measurements pose some more challenges. Permeability prediction using equations (6.21) - (6.23) require an accurate measurement of the intrinsic formation factor. Additionally, use of equation (6.21) also requires the porosity for the calculation of the cementation exponent. This may not be easy. Except in the case of highly saline aquifers, the measured formation factor will be an under-estimate of the intrinsic 
formation factor. This necessitates the need for some alternate methods for correcting the formation factor. Equation (6.24) has the advantage that it does not depend on formation factor and porosity. However, the constants estimated in the laboratory may not be applicable for the field measurements.

\subsection{Dependence of SIP on pore fluid conductivity - further observations}

The Series 1 and Series 2 samples were saturated with pore water of a given conductivity by passing about 20 litres of fluid through the sample for determining the hydraulic conductivity. This takes about 10-20 minutes depending on the grain size of the sample. Normally SIP measurements are made on the sample after a further period of at least 48 hours. However, if continuous measurements are made during this 48 -hour period a gradual increase in the pore water conductivity can be inferred from the fact that the electrical conductivity of the sample keeps increasing. This variation in the conductivity of the sample may be a result of the dissolution of ions into the pore water from the minerals in the sand.

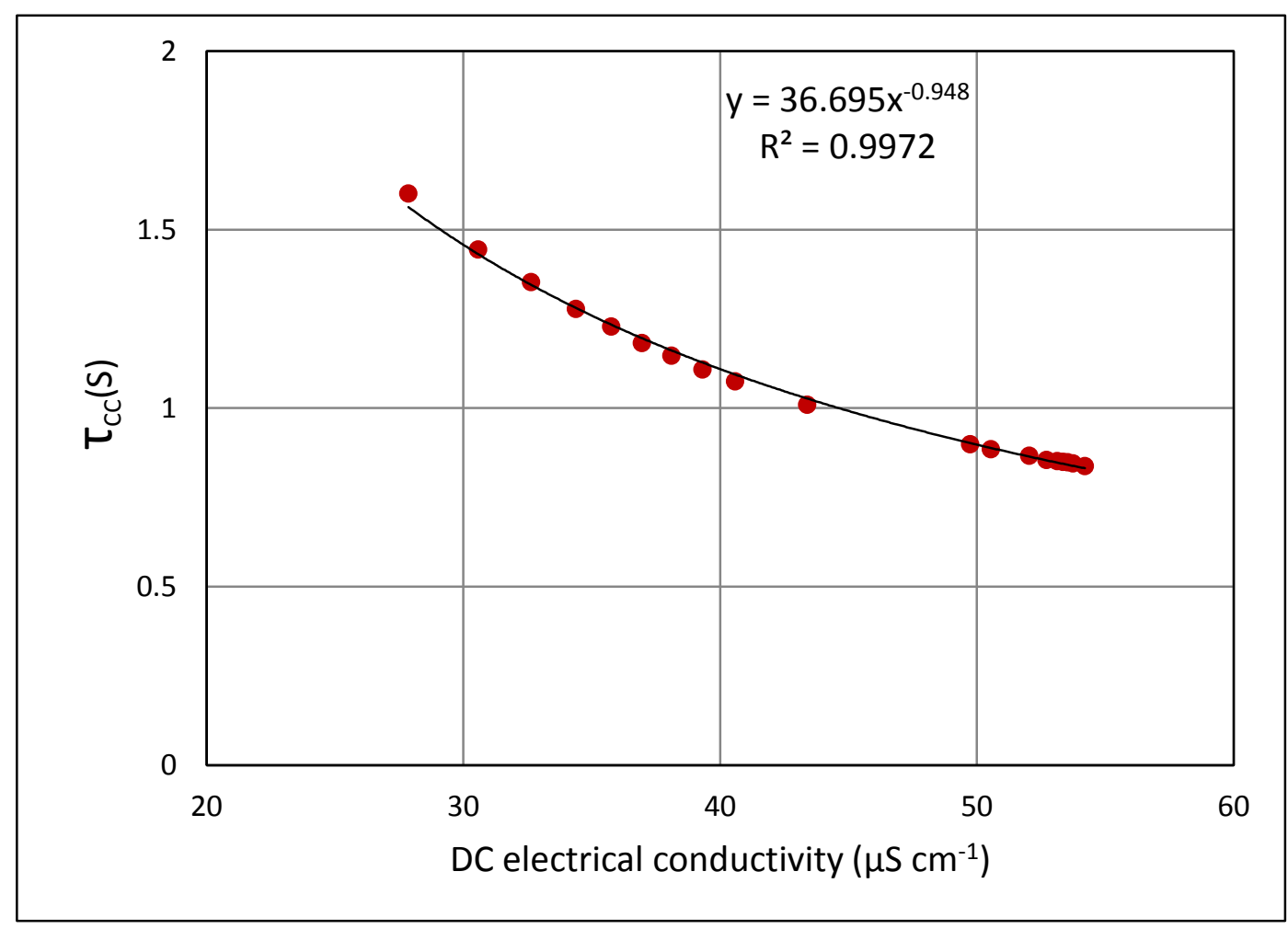

Figure 6.17: Cole-Cole relaxation time constant plotted against the DC electrical resistivity for the sample $\varphi=2.5$ in Series 2 . 
As the conductivity increases, there is a corresponding decrease in the value of the ColeCole relaxation time constant as shown in Figure 6.17. Such a variation has been observed for all the samples which are described as Series 1 and Series 2. But the extent of variation in pore water conductivity, and hence the Cole-Cole relaxation time constant, varies among samples. It should be noted that a somewhat significant variation in Cole-Cole relaxation time occurs only at pore water conductivities below 100 $\mu \mathrm{Scm}^{-1}$. For some of the measurements, but not all, this variation can be well represented by a power law. Using the power law equation that fits the data for the sample with $\varphi=2.5$ in Series 2 , an attempt has been made to extrapolate the value of Cole-Cole relaxation time to lower and higher conductivities of pore water. The result of one such fit for data presented in Figure 6.17 is shown in Figure 6.18. While it cannot be assured that the relationship observed in a short range of pore fluid conductivity holds good for a much larger range, it can be reasonably concluded that at higher salinities there is not much effect on the estimated Cole-Cole relaxation time; while it can be significant at lower conductivities. As the pore water conductivity of all samples when

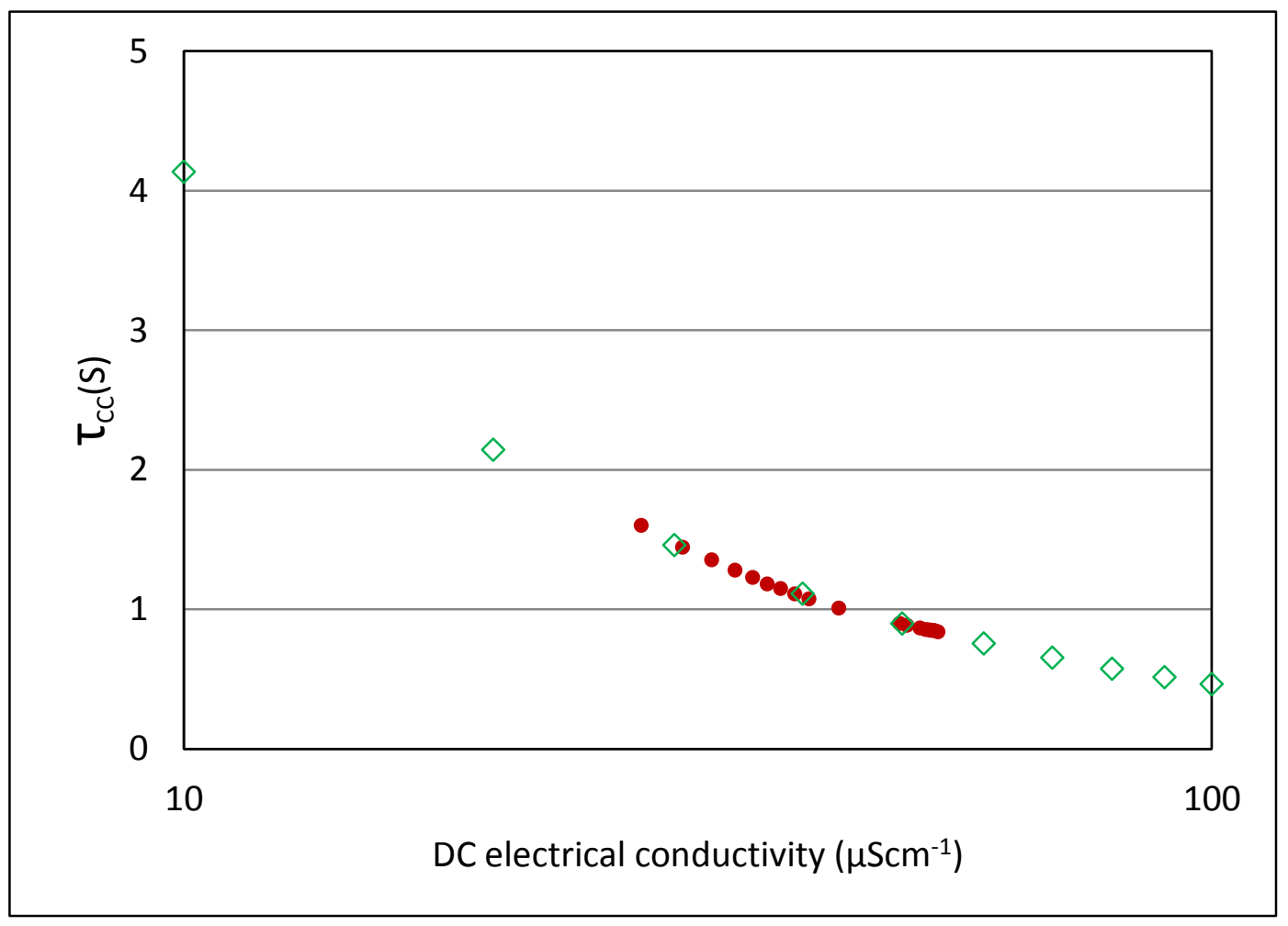

Figure 6.18: Cole-Cole relaxation time constant $\left(\tau_{C C}\right)$ plotted against DC electrical resistivity for the sample $\varphi=2.5$ in Series 2 . The circles are experimentally determined values while the diamonds are calculated using the power equation (shown in Figure 6.17) that fit the experimentally measured data. 
their SIP spectra was recorded was above $100 \mu \mathrm{Scm}^{-1}$ the impact of variation in ColeCole relaxation time due to slightly different fluid conductivities of the sample is inferred to be insignificant.

\subsection{Conclusion}

SIP and hydraulic conductivity measurements were made on sieved fractions of sand and mixtures of sieved fractions of sand in various proportions. The measured hydraulic conductivity shows a good correlation to the grain size, relaxation time constant and hydraulic conductivity predicted by various relationships. While none of these relationships can be taken as a universal predictor for hydraulic conductivity the correlation displayed suggests that the relaxation time constant is sensitive to the variation of hydraulic conductivity due to variation in grain size. In many cases the difference between the measured and predicted permeability is small though this close prediction is not sufficient to conclude that precise predictions are necessarily possible. However, it is promising to see the excellent sensitivity of the relaxation time constant to the hydraulic conductivity in the case of mixtures of sieved fractions of sand. This is a strong indicator the relaxation time will be a good predictor for hydraulic conductivity for natural samples which have a distribution of grain sizes.

For the samples prepared in the laboratory the best prediction of permeability comes from equation (6.16). It has to be kept in mind that these samples are very well sorted and hence it is not surprising that equation (6.16) is able to give good predictions. Equation (6.18) may be more appropriate to predict the permeability of more densely packed media like sandstones. The purpose of this study was to see how well the relaxation time constant can replace grain size in an equation that predicts permeability. It is in this context that equations (6.21) and (6.22) are tested. The constants involved take care of all factors that influence permeability other than the grain size/pore size. Although it appears that developing a single universal equation to predict permeability may not be possible, it is beyond any doubt that SIP parameters can give an estimate of grain/pore size at least in unconsolidated sediments. It is to be investigated that how much this can be extended to other natural pores systems. 


\section{Summary}

This thesis reports on an investigation into the possibility of using the Spectral Induced Polarization (SIP) method for the determination of hydraulic conductivity of materials that are typically found in New Zealand shallow coastal aquifers. The ability of the SIP response to provide additional information about the grain-pore fluid interface has been known for a long time. SIP was in use from the 1950s but except for one or two attempts there was not much effort to use it for hydrogeophysical applications. The fact that the SIP method has not yet found its place as a viable tool in hydrogeophysics and in prominent geophysical organisations, as there are only very few manufactures of SIP instrumentation. The very low polarizability of many geological materials, especially those which do not have metallic minerals, demands a very good quality of impedance spectrometer that can measure a phase difference as small as few milliradians.

The custom built impedance spectrometer developed at Victoria University of Wellington has proved to be a success in making laboratory measurements on unconsolidated sand samples. Although the upper limits of $20 \mathrm{mApp}_{\mathrm{p}}$ in current, and $12 \mathrm{~V}_{\mathrm{pp}}$ in voltage, restricts the use of the impedance spectrometer in the field. The next generation instrument which can provide $1 A_{p p}$ and $70 V_{p p}$ respectively in current and voltage, will be able to address this drawback.

The measurements made on two series of sieved fractions of sand and mixtures of different fractions are encouraging. The correlation between the Cole-Cole relaxation time constant recovered by fitting the measured SIP response with a Cole-Cole model shows an excellent fit to the expected relationship with grain size. They are very well explained by a power law fit and the power of the fit is found to be close to 2 . The results also show that the Cole-Cole relaxation time constant is extremely sensitive to the presence of smaller grains in a well sorted sand.

Four different models for predicting the permeability from SIP spectra have been tested on the data obtained in the present study. All the models show a good fit, although the goodness of fit varies. The predicting ability of the models was also tested on the data available in the literature. It should be noted that very few data are available on SIP measurements on unconsolidated sediments. The results of the present study show that the pore fluid conductivity at which measurements are conducted can influence the 
permeability prediction. This is caused by a slight influence on relaxation time constant, and a much bigger influence through the formation factor. Such variations may have very little relevance in a field setting but could be important when making accurate measurements in the laboratory to investigate the polarization mechanisms. The presence of the electrical formation factor, and its dependence on the pore fluid conductivity, means that these models should be used with caution. This is because the formation factor estimated for freshwater will be definitely an under-estimate of the intrinsic formation factor. By adding a single multiplicative constant as a correction term all the models are able to give an excellent fit to measured values of hydraulic conductivity. This shows that irrespective of the excellent correlation between permeability and the Cole-Cole relaxation time recovered from the fitting of measured SIP responses to the Cole-Cole model, predicting the exact hydraulic conductivity is difficult. This means that there may not be a universal model that relates an SIP response to hydraulic conductivity.

Notwithstanding this there is no doubt that SIP is a viable method to determine the hydraulic conductivity of New Zealand shallow coastal aquifers. It is interesting to note that the all samples used in the study have a much larger SIP response compared to that of pure silica sand. This is something that needs further investigation and may potentially throw light on the polarization of the electrical double layer. If this large SIP response is characteristic of most of the aquifer materials, then this is a factor that further encourages the use of SIP on New Zealand shallow coastal aquifers.

This is the first work reported on SIP measurements made on New Zealand sands, whilst there are very few work published on SIP measurements on unconsolidated sediments. This work represents the first measurements on sands that have been well sieved and in the range of grain size $1000 \mu \mathrm{m}$ to $125 \mu \mathrm{m}$. This project has also further developed and established the construction of a simple sample holder that permits the SIP and hydraulic conductivity measurements to be made without the need to repack the sample as occurs in many studies reported in the literature. The use of a low cost custom made impedance spectrometer is another highlight. Such low cost instruments can help in promoting more work in this field.

All the samples measured during this study except the pure silica sand sample showed a SIP response much larger than that for typical silica sands. This may indicate the 
presence of minerals that can enhance the SIP response, but also is partly due to the use of pore fluids of relatively low conductivity. However this observation indicates that a detailed study including characterising the mineralogy of the samples is necessary.

The SIP measurements on mixtures with a systematic variation in their grain size fraction is the first of its kind. While this study has presented a general trend in the results it is recommended that there is further investigation to try and establish a model that explains how the SIP response of different grain sizes combine/average out to form a resultant spectrum and whether that can give an insight into the grain size distribution.

The author recommends to extend the measurements on the impact of compaction on SIP spectra. Combining the result of such a study with the current measurements can throw light on whether it is the grain size or pore size that controls the SIP mechanism in unconsolidated sediments, or more importantly, whether there is a transformation from a grain size controlled phenomenon to pore size based phenomenon at some critical compaction level.

While it has not been recorded and reported in this thesis it was observed that temperature does affect the relaxation time. As measurements for this study were made at constant temperature the impact of such a change is negligible in the present data, but it underlines the importance of maintaining the temperature constant during SIP measurements where the expected change in SIP spectra is small.

Another interesting study will be the influence of salinity on SIP spectra at very low and very high salinity levels. It may be very difficult to maintain the salinity steady at salinity levels less than $30 \mu \mathrm{Scm}^{-1}$ in the case of natural samples as they may contain dissolvable minerals. This can be overcome by the use of chemically stable spherical objects like glass beads. 


\section{Appendix 1}

\section{Table of sampling rates for data acquisition}

\begin{tabular}{|c|c|c|c|c|c|}
\hline \multirow[t]{2}{*}{ Serial No. } & \multirow[t]{2}{*}{ Frequency in $\mathrm{Hz}$} & \multirow[t]{2}{*}{ Sampling rate $\mathrm{S} / \mathrm{s}$} & \multicolumn{3}{|c|}{ No. of Samples collected for } \\
\hline & & & 1 Cycle & 2 Cycles & 3 Cycles \\
\hline 1 & 1000 & 23997.07067 & 131072 & 262144 & 393216 \\
\hline 2 & 794.3 & 23999.65182 & 131072 & 262144 & 393216 \\
\hline 3 & 631 & 23993.74296 & 131072 & 262144 & 393216 \\
\hline 4 & 501.2 & 23993.16523 & 131072 & 262144 & 393216 \\
\hline 5 & 398.1 & 23990.69572 & 131072 & 262144 & 393216 \\
\hline 6 & 316.2 & 23998.24343 & 131072 & 262144 & 393216 \\
\hline 7 & 251.2 & 23998.02216 & 131072 & 262144 & 393216 \\
\hline 8 & 199.5 & 23989.78349 & 131072 & 262144 & 393216 \\
\hline 9 & 158.5 & 23989.50577 & 131072 & 262144 & 393216 \\
\hline 10 & 125.9 & 23985.41395 & 131072 & 262144 & 393216 \\
\hline 11 & 100 & 23961.97441 & 131072 & 262144 & 393216 \\
\hline 12 & 79.43 & 23988.59207 & 131072 & 262144 & 393216 \\
\hline 13 & 63.1 & 23972.87884 & 131072 & 262144 & 393216 \\
\hline 14 & 50.12 & 23975.65197 & 131072 & 262144 & 393216 \\
\hline 15 & 39.81 & 23935.67119 & 131072 & 262144 & 393216 \\
\hline 16 & 31.62 & 23956.62798 & 131072 & 262144 & 393216 \\
\hline 17 & 25.12 & 23858.90319 & 131072 & 262144 & 393216 \\
\hline 18 & 19.95 & 23989.78349 & 131072 & 262144 & 393216 \\
\hline 19 & 15.85 & 23879.2092 & 131072 & 262144 & 393216 \\
\hline 20 & 12.59 & 23915.89101 & 131072 & 262144 & 393216 \\
\hline 21 & 10 & 23831.27273 & 131072 & 262144 & 393216 \\
\hline 22 & 7.943 & 23661.47491 & 131072 & 262144 & 393216 \\
\hline 23 & 6.31 & 23630.40914 & 131072 & 262144 & 393216 \\
\hline 24 & 5.012 & 23461.888 & 131072 & 262144 & 393216 \\
\hline 25 & 3.981 & 23718.07418 & 131072 & 262144 & 393216 \\
\hline 26 & 3.162 & 23024.98133 & 131072 & 262144 & 393216 \\
\hline 27 & 2.512 & 23518.06171 & 131072 & 262144 & 393216 \\
\hline 28 & 1.995 & 23771.69455 & 131072 & 262144 & 393216 \\
\hline 29 & 1.585 & 23083.23556 & 131072 & 262144 & 393216 \\
\hline 30 & 1.259 & 23574.23543 & 131072 & 262144 & 393216 \\
\hline 31 & 1 & 21845.33333 & 131072 & 262144 & 393216 \\
\hline 32 & 0.7943 & 20822.09792 & 131072 & 262144 & 393216 \\
\hline 33 & 0.631 & 20676.608 & 131072 & 262144 & 393216 \\
\hline 34 & 0.5012 & 21897.76213 & 131072 & 262144 & 393216 \\
\hline 35 & 0.3981 & 17393.2544 & 131072 & 262144 & 393216 \\
\hline 36 & 0.3162 & 20722.4832 & 131072 & 262144 & 393216 \\
\hline 37 & 0.2512 & 16462.6432 & 131072 & 262144 & 393216 \\
\hline 38 & 0.1995 & 13074.432 & 131072 & 262144 & 393216 \\
\hline 39 & 0.1585 & 20774.912 & 131072 & 262144 & 393216 \\
\hline 40 & 0.1259 & 16501.9648 & 131072 & 262144 & 393216 \\
\hline
\end{tabular}




\begin{tabular}{|l|l|l|l|l|l|}
\hline Serial No. & Frequency in Hz & Sampling rate S/s & \multicolumn{3}{|c|}{ No. of Samples collected for } \\
\cline { 4 - 6 } & & & $\mathbf{1}$ Cycle & $\mathbf{2}$ Cycles & 3 Cycles \\
\hline 42 & 0.07943 & 10411.04896 & 131072 & 262144 & 393216 \\
\hline 43 & 0.0631 & 8270.6432 & 131072 & 262144 & 393216 \\
\hline 44 & 0.05012 & 6569.32864 & 131072 & 262144 & 393216 \\
\hline 45 & 0.03981 & 5217.97632 & 131072 & 262144 & 393216 \\
\hline 46 & 0.03162 & 4144.49664 & 131072 & 262144 & 393216 \\
\hline 47 & 0.02512 & 3292.52864 & 131072 & 262144 & 393216 \\
\hline 48 & 0.01995 & 2614.8864 & 131072 & 262144 & 393216 \\
\hline 49 & 0.01585 & 2077.4912 & 131072 & 262144 & 393216 \\
\hline 50 & 0.01259 & 1650.19648 & 131072 & 262144 & 393216 \\
\hline 51 & 0.01 & 1310.72 & 131072 & 262144 & 393216 \\
\hline 52 & 0.007943 & 1041.104896 & 131072 & 262144 & 393216 \\
\hline 53 & 0.00631 & 827.06432 & 131072 & 262144 & 393216 \\
\hline 54 & 0.005012 & 656.932864 & 131072 & 262144 & 393216 \\
\hline 55 & 0.003981 & 521.797632 & 131072 & 262144 & 393216 \\
\hline 56 & 0.003162 & 414.449664 & 131072 & 262144 & 393216 \\
\hline 57 & 0.002512 & 329.252864 & 131072 & 262144 & 393216 \\
\hline 58 & 0.001995 & 261.48864 & 131072 & 262144 & 393216 \\
\hline 59 & 0.001585 & 207.74912 & 131072 & 262144 & 393216 \\
\hline 60 & 0.001259 & 165.019648 & 131072 & 262144 & 393216 \\
\hline 61 & 0.001 & 131.072 & 131072 & 262144 & 393216 \\
\hline
\end{tabular}




\section{Appendix 2}

\section{Publications and conference presentations relating to this research}

\section{Publications}

1. Joseph, S., Ingham, M. \& Gouws, G., 2015. Spectral Induced Polarization measurements on New Zealand Sands - dependence on fluid conductivity. Near Surface Geophysics, 2015, 13 169-177.

http://nsg.eage.org/publication/publicationdetails/?publication=78521

\section{Conference Presentations}

1. Joseph, S., Ingham, M. \& Gouws, G., 2012. Spectral Induced polarization measurements on New Zealand sands. Fall Meeting, American Geophysical Union, San Francisco, 3-7 December 2012.

2. Joseph, S., Ingham, M. \& Gouws, G., 2013. Spectral Induced Polarization Measurements on New Zealand Sands - Dependence on Fluid Conductivity. Proceedings of Near Surface Geoscience 2013 - 19th European Meeting of Environmental and Engineering Geophysics Bochum, Germany, 9-11 September 2013.

http://earthdoc.eage.org/publication/publicationdetails/?publication=70924

3. Joseph, S., Ingham, M. \& Gouws, G., 2013. Spectral induced polarization (SIP) measurements on New Zealand unconsolidated. Proceedings of The NZ hydrological society and The meteorological society of NZ joint conference Palmerston North, 19-22 November 2013. Pages 108-109.

4. Ingham, M. Joseph, S., \& Gouws, G., 2013 IP and SIP - The missing link to hydraulic conductivity. Proceedings of The NZ hydrological society and The meteorological society of NZ joint conference Palmerston North, 19-22 November 2013. Pages 102-103 
5. Joseph, S., Ingham, M. \& Gouws, G., 2014. Spectral induced polarization and hydraulic conductivity measurements on New Zealand unconsolidated sediments. Proceedings of $3^{\text {rd }}$ international workshop on induced polarization, I le dOleron, Charente-Maritime, France, 6-9 April 2014.

http://ip.geosciences.mines-paristech.fr/s1 joseph

6. Ingham, M. Joseph, S., K. Ilse \& Gouws, G., 2014. IP and SIP the practical link? Proceedings of $3^{\text {rd }}$ international workshop on induced polarization, I le dOleron, Charente-Maritime, France, 6-9 April 2014.

http://ip.geosciences.mines-paristech.fr/s1 ingham

7. Joseph, S. Ingham, M., 2014. Spectral Induced Polarization and hydraulic properties of New Zealand Sands. Fall meeting, American Geophysical Union, San Francisco, 15-19 Dec 2014. 


\section{References}

Angelos, N. F. (2011), Highlights and common themes of groundwater management practices around the World, in Groundwater Management Practices, edited, pp. 1-16, CRC Press.

Archie, G., E., (1942), The electrical resistivity log as an aid indetermining some reservoir characteristics., Transactions of the American Institute of Mining and Metallurgical Engineers, 146(1), 54-62.

Avellaneda, M., and S. Torquato (1991), Rigourous link between fluid permeability, electrical conductivity and relaxation times for transport in porous media, Physics of Fluids A, 3(11), 2529-2540.

Bairlein, K., A. Hördt, and S. Nordsiek (2014), The influence of sample preparation on spectral induced polarization of unconsolidated sediments, Near Surf Geophys, 12(5), 667-677.

Barker, R., D. (2007), Electrical Resistivity Methods for Borehole Siting in Hardrock Region, in Groundwater Resource Evaluation, Augmentation, Contamination, Restoration, Modeling and Management, edited by M. Thangarajan, pp. 26-60, Springer, Dordrecht.

Bear, J. (1972), Dynamics of Fluids in Porous Media, American Elsevier Publishing Company Inc., New York.

Beck, A., E. (1981), Physical Principles of Exploration Methods, THE MACMILLAN PRESS LTD.

Binley, A., and A. Kemna (2005), DC Resistivity and Induced Polarization methods, in Hydrogeophysics, edited by Y. Rubin and S. S. Hubbard, pp. 129-156, Springer, Dordrecht.

Binley, A., L. Slater, D., K. Fukes, and G. Cassiani (2005), Relationship between spectral induced polarization and hydraulic properties of saturated and unsaturated sandstone, Water Resources Research, 41(12), W1247.

Binley, A., S. Kruschwitz, D. Lesmes, and N. Ketteridge (2010), Exploiting the temperature effects on low frequency electrical spectra of sandstone: a comparison of effective diffusion path lengths, Geophysics, 75(6), A43-A46.

Bleil, D., F. (1953), Induced Polarization: A Method of Geophysical Prospecting, Geophysics, 18(3), 636-661. 
Boadu, F. K. (2000), Hudraulic Conductivity of Soils from Grain-Size Distribution: New Models, Journal of Geotechnical and Environment Engineering, 126(8), 739-746.

Börner, F., D. (1991), Untersuchungen zur komplexen elektrischen Leitfähigkeit von Gesteinen im Frequenzbereich von 1 Milliherz bis 10 Kiloherz, Unpublished Dissertation, Bergakademie Freiberg, Germany. Börner, F., D. (1992), Complex Conductivity Measurements of Reservoir Properties, in Advances in Core Evaluation III Reservoir Management, edited by P. Worthington, F. and C. Chardaire-Rivière, Gordon and Breach Science Publishers.

Börner, F., D., J. Schopper, R., and A. Weller (1996), Evaluation of transport and storage properties in the soil and groundwater zone from induced polarization measurements, Geophysical Prospecting, 44(4), 583-601.

Breede, K., A. Kemna, O. Esser, E. Zimmermann, H. Vereecken, and J. Huisman, A. (2012), Spectral induced polarization measurements on variably saturated sand-clay mixtures, Near Surf Geophys, 10(6), 479-489.

Burger, R. H., A. F. Sheehan, and C. H. Jones (2006), Introduction to Applied Geophysics, W. N. Norton \& Company, New York.

Bussian, A. E. (1983), Electrical conductance in a porous medium, Geophysics, 48(9), 1258-1268.

Cech, T. V. (2003), Principles of water resources: history, development, managent and policy, Wiley, New York.

Chapuis, R., P., and M. Aubertin (2003), On the use of the Kozeny-Carman equationh to predict the hydraulic conductivity of soils, Canadian Geotechnical Journal, 40(3), 616628.

Chelidze, T., L., and Y. Gueguen (1999), Electrical spectroscopy of porous rocks: a review - I. Theoretical models, Geophys J Int, 137(1), 1-15.

Chilton, J. (1992), Groundwater, in Water Quality Assessments - A Guide to Use of Biota, Sediments and Water in Environmental Monitoring - Second Edition, edited by D. Chapman, E\&FN Spon, London.

Cole, K., S., and R. Cole, H. (1941), Dispersion and Absorption in Dielectrics I. Alternating Current Characteristics, Journal of Chemical Physics, 9, 341 - 351. Custodio, E. (2002), Aquifer overexploitation: What does it mean?, Hydrogeology Journal, 10(2), 254-277. 
Dahlin, T., and M. Loke, H. (1997), Quasi-3D resistivity imaging -mapping of three dimensional structures using two dimensional structures using two dimensional DC resistivity techniques, Procs. 3rd meeting Environmental and Engineering Geophysics, Aarhus, Denmark, 8-11 September 1997, 143-146.

Davidson, D. W., and R. Cole, H. (1951), Dielectric Relaxation in Glycerol, Propylene Glycol and n-propanol, The Journal of Chemical Physics, 19(12).

de Lima, O. A., and M. M. Sharma (1992), A generalized Maxwell-Wagner theory for membrane polarization in shaly sands., Geophysics, 57(3), 431-440.

Debye, P. (1929), Polar Molecules, Chemical Catalog Company, New york.

Dias, C., A. (2000), Developments in a model to describe low-frequency electrical polarization of rocks, Geophysics, 65(2), 437-451.

Eggleston, J., and S. Rojstaczer (2001), The Value of Grain-size Hydraulic Conductivity Estimates: Comparison with High Resolution In-situ Field Hydraulic Conductivity, Geophysical Research Letters, 28(22).

Florsch, N., M. Llubes, E. Tereygeol, A. Ghorbani, and P. Roblet (2011), Quantification of slag heap volumes and masses through the use of induced polarization: application to the Castel-Minier site, J Archaeol Sci, 38(2), 438-451.

Fox, W., Robert (1830), On the Electro-Magnetic Properties of Metalliferous Veins in the Mines of Cornwall, Philos. Trans. R. Soc. Lond., 120, 399-414.

Guéguen, Y., and V. Palciauskas (1994), Introduction to the physics of rocks, Princeton University Press.

Hales, T. C. (2005), A proof of the Kepler conjecture, Annals of Mathematics. Second Series,, 162(3), 1065-1185.

Hawke, R., M., and J. A. McConchie (2005), The source, age, and stabilization of Kaputaroa dunes, Otaki-Te Horo, New Zealand Journal of Geology and Geophysics, 48, 517-522.

Hawke, R., M., and J. A. McConchie (2006), Dune Phases in the Otaki-Te Horo area ( New Zealand): a geomorphic history, Earth Surface Processes and Landforms, 31, 633645.

Hördt, A., R. Blaschek, A. Kemna, and N. Zisser (2007), Hydraulic conductivity estimation from induced polarization data at the field scale - the Krauthausen case history, Journal of Applied Geophysics, 62(1), 33-46. 
Hunt, C., P., B. Moskowitz, M., and S. Banerjee, K. (1995), Magnetic properties of Rocks and minerals, in Rock Physics and Phase relations. A handbook of Physicl Constants., edited, pp. 189-204, American Geophysical Union.

Huntley, D. (1986), Relations Between Permeability and Electrical Resistivity in Granular Aquifers, Groundwater, 24(4), 466-474.

Ingham, M., G. Gouws, S. Buchanan, R. Brown, and T. Haskell (2012), In-situ measurements of the low frequency dielectric permittivity of first-year Antartic sea ice, Cold Regions Science and Technology, 83-84, 139-146.

Johnson, D. L., J. Koplik, and L. M. Schwarz (1986), New pore-size parameter characterizing transport in porous media, Physical Review Letters, 57(20), 2564-2567. Joseph, S., M. Ingham, and G. Gouws (2015), Spectral Induced Polarization measurements on New Zealand sands - dependence on fluid conductivity, Near Surf Geophys, 13(2), 169-177.

Keller, G., V., and F. Frischknecht, C. (1966), Electrical Methods in Geophysical Prospecting, Pergamon Press Inc.

Kemna, A. (2000), Tomographic inversion of complex resistivity: theory and application, PhD thesis, Bochum University.

Kemna, A., et al. (2012), An overview of the spectral induced polarization method for near-surface applications, Near Surf Geophys, 10(6), 453-468.

Koch, K., A. Revil, and K. Holliger (2012), Relating the permeability of quartz sands to their grain size and spectral induced polarization characteristics, Geophys J Int, 190(1), 230-242.

Koltermann, C., E., and S. Gorelick, M. (1995), Fractional packing model for hydraulic conductivity derived from sediment mixtures, Water Resources Research, 31(12), 3283-3297.

Krumbein, W. C., and E. Aberdeen (1937), The Sediments of Barataria Bay, Journal of Sedimentary Petrology, 7(3-17).

Kruschwitz, S., A. Binley, D. Lesmes, and A. Elshenawy (2010), Textural controls on lowfrequency electrical spectra of porous media, Geophysics, 75(4), WA113-WA123.

Leroy, P., A. Revil, A. Kemna, P. Cosenza, and A. Ghorbani (2008), Complex conductivity of water-saturated packs of glass beads, Journal of Colloid and Interface Science, 321(1), 103-117.

Loke, M., H. (1999), A practical guide to 2-D and 3-D surveys. 
Loke, M., H. (2004), Tutorial : 2-D and 3-D electrical imaging surveys.

Loke, M., H., and R. Barker, D. (1996), Practical technique for 3D resistivity surveys and data inversion, Geophysical Prospecting, 44, 499-523.

Mansoor, N., and L. Slater (2007), On the relationship between iron concentration and induced polarization in marsh soils., Geophysics, 72(1), A1-A5.

Masch, F., D., and K. Denny, J. (1966), Grain Size Distribution and its Effects on The Permeability of Unconsolidated Sands, Water Resources Research, 2(4), 665-677. Mathews, P., and K. Zonge, L. (2003), 50 years State of the art in IP and complex resistivity, in KEGS 50th Anniversary Symposium Mining and Environmental Geophysics - Past,Present \&Future, edited, Toronto, Ontario.

Nielsen, D., M. (1991), Practical Handbook of Ground-Water Monitoring, Lewis Publishers.

Nordsiek, S., and A. Weller (2008), A new approach to fitting induced polarization spectra, Geophysics, 73(6), F235-F245.

O’Konsky, C. T. (1960), Electric properties of macromolecules. V. Theory of ionic polarization in polyelectrolytes., Journal of Physical Chemistry, 64, 605-619.

Osborne, A. M. (2006), Movement of water within the Waikanae Shallow Gravel Aquifer and its Interaction with the Waikanae River., Unpublished MSc thesis, School of Geography, Environmental and Earth Sciences, Victoria University of Wellington, Wellington, $165 p$.

Pelton, W., H., W. Sill, R., and B. Smith, D. (1983), Interpretations of complex resistivity and dielectric data, Part I, Geophysical Transactions, 29, 297-330.

Pelton, W., H., S. H. Ward, P. Hallof, G, W. Sill, R., and P. Nelson, H. (1978), Mineral Discrimination and Removal of Inductive Coupling with Multifrequency IP, GEOPHYSICS, 43(3), 588-609.

Porter, L., B., R. Ritzi, W., L. Mastera, J., D. Dominic, F., and B. Ghanbarian-Alavijeh (2013), The Kozeny-Carman Equation with a Percolation Threshold, Groundwater, 51(1), 92-99.

Revil, A., and P. W. J. Glover (1997), Nature of surface electrical conductivity in natural sands, sandstones and clays. , Geophysical Research Letters, 25, 691-694.

Revil, A., and N. Florsch (2010), Determination of permeability from spectral induced polarization in granular media, Geophys J Int, 181(3), 1480-1498. 
Revil, A., and P. Cosenza (2010), Comment on 'Generalized effective-medium theory of induced polarization' (Michael Zhdanov, 2008, Geophysics 73(2), F197-F211), Geophysics, 75(2), X7-X9.

Revil, A., and M. Skold (2011), Salinity dependence of spectral induced polarization in sands and sandstones., Geophys J Int, 187(2), 813-824.

Revil, A., K. Koch, and K. Holliger (2012a), Is it the grain size or the characteristic pore size that controls the induced polarization relaxation time of clean sands and sandstone?, Water Resources Research, 48(5).

Revil, A., K. Titov, C. Doussan, and V. Lapenna (2006), Application of self-potential method to hydrological problems, in Applied Hydrogeophysics, edited by H. Vereecken, A. Binley, G. Cassiani, A. Revil and K. Titov, Springer, Dordrecht.

Revil, A., M. Karaoulis, T. Johnson, and A. Kemna (2012b), Review: Some low-frequency electrical methods for subsurface characterization and monitoring in hydrogeology, Hydrogeology Journal, 20(4), 617-658.

Revil, A., J. Eppehhimer, D., M. Skold, M. Karaoulis, L. Godinez, and M. Prasad (2013), Low-frequency complex conductivity of sand and clayey materials, Journal of Colloid and Interface Science, 398, 193-209.

Reynolds, J. M. (2011), An introduction to applied and environmental geophysics, 2nd ed., xiii, 696 p. pp., Wiley-Blackwell, Chichester, West Sussex ; Malden, Mass. Rosas, J., O. Lopez, T. Missimer, M., K. Coulibaly, M., A. Dehwah, H.A., K. Sesler, L. Lujan, R., and D. Mantilla (2014), Determination of Hydraulic conductivity from GrainSize Distribution for Different Depositional Environments, Groundwater, 52(3), 399413.

Rubin, Y., and S. S. Hubbard (2005), Introduction to Hydrogeophysics, in Hydrogeophysics, edited by Y. a. H. Rubin, Susan S., Springer, Dordrecht. Schurr, J., M. (1964), On the theory of the dielectric dispersion of spherical colloidal particles in electrolytic solutions, Journal of Physical Chemistry, 68, 2407-2413. Schwarz, G. (1962), A theory of the low-frequency dielectric dispersion of colloidal particles in electrolyte solutions, Journal of Physical Chemistry, 66, 2636-2642.

Scott, G., D. (1960), Packing of spheres, Nature, 188, 908-909.

Scott, G., D. , and D. M. Kilgour (1969), The density of random close packing of spheres, Journal of Physics D, 2, 863-866. 
Seidel, K., and G. Lange (2007), Direct Current Resistivity Methods, in Environmental Geology Handbook of Field Methods and Case Studies, edited by K. Knödel, G. Lange and H.-J. Voigt, Springer.

Seigel, H., O., M. Nabighian, D. Parasnis, S., and K. Vozoff (2007), The early history of induced polarization method, The Leading Edge, 26(3), 312-321.

Slater, L., D. (2007), Near surface electrical characterization of hydraulic conductivity: from petrophysical properties to aquifer geometries - a review, Surveys in Geophysics, 28(2-3), 169-197.

Slater, L., D., and D. Lesmes (2002), IP interpretations in environmental investigations, Geophysics, 67(1), 77-88.

Slater, L., D., and D. Glaser, R. (2003), Controls on induced polarization in sandy unconsolidated sediments and application to aquifer chracterization, Geophysics, 68(5), 1547-1558.

Slater, L., D., W. Barrash, J. Montrey, and A. Binley (2014), Electrical-hydraulic relationships observed for unconsolidated sediments in the presence of a cobble framework, Water Resources Research, 50, 5721-5742.

Sumner, J. S. (1976), Principles of induced polarization for geophysical exploration, Elsevier Scientific Publishing Company.

Szalai, S., and L. Szarka (2008), On the classification of surface geoelectric arrays, Geophysical Prospecting, 56, 159-175.

Tarasov, A., and K. Titov (2013), On the use of Cole-Cole equations in spectral induced polarization, Geophys J Int, 195(1), 352-356.

Telford, W. M., L. P. Geldart, R. E. Sheriff, and D. A. Keys (1976), Applied Geophysics, Cambrdge University Press, London.

Titov, K., V. Komarov, V. Tarasov, and A. Levitski (2002), Theoretical and experimental study of time domain-induced polarization in water-saturated sands, Journal of Applied Geophysics, 50(4), 417-433.

Todd, D. K. (1959), Groundwater Hydrology.

Tong, M., and H. Tao (2008), Permeability estimating from complex resistivity measurement of shaly sand reservoir, Geophys J Int, 173(2), 733-739.

Tong, M., L. Li, W. Wang, and Y. Jiang (2006a), Determining capillary-pressure curve, pore-size distribution and permeability from induced polarization of shaley sand., Geophysics, 71(3), N33-N40. 
Tong, M., L. Li, W. Wang, and Y. Jiang (2006b), A time-domain induced-polarization method for estimating permeability in a shaly sand reservoir, Geophysical Prospecting, 54(5), 623-631.

Tsourlos, P., R. Ogilvy, C. papazachos, and P. Meldrum (2011), Measurement and inversion schemes for single borehole-to-surface electrical resistivity tomography surveys, JOURNAL OF GEOPHYSICS AND ENGINEERING, 8, 487-497.

Vacquier, V., C. Holmes, R., P. Kintzinger, R., and M. Lavergne (1957), Prospecting for ground water by induced electrical polarization, Geophysics, 22(3), 660-687.

Van Nostrand, R. G., and K. L. Cook (1966), Interpretation of Resistivity Data, United States Geological Survey Professional Paper, 499.

Vineagar, H., J., and M. Waxman, H. (1984), Induced polarization of shaly sands, Geophysics, 49(8), 1267-1287.

Wait,James, R., (1959), Overvoltage Research and Geophysical Applications, Pergamon Press.

Ward, S. H. (1990), Resistivity and Induced Polarization methods, in Geotechnical and Environmental geophysics, Volume 1 : Review and Tutorial, edited by S. H. Ward, pp. 147-189, Society of Exploration Geophysicists.

Weller, A., L. Slater, D., and S. Nordsiek (2013), On the relationship between induced polarization and surface conductivity: Implications for petrophysical interpretation of electrical measurements, Geophysics, 78(5), D315-D325.

Weller, A., L. Slater, S. Nordsiek, and D. Ntarlagiannis (2010), On the estimation of specific surface per unit pore volume from induced polarization: A robust empirical relation fits multiple data sets Geophysics, 75(4), WA105-WA112.

Weller, A., K. Breede, L. Slater, D., and S. Nordsiek (2011), Effect of changing water salinity on complex conductivity spectra of sandstones, Geophysics, 76(5), F315-F327. Weller, A., L. Slater, A. Binley, S. Nordsiek, and S. Xu (2015), Permeability prediction based on induced polarization: insights from measurements on sandstone and unconsolidated samples spanning a wide permeability range, Geophysics, 80(2), D161D173.

Whiteley, R., J. (1973), Electrode arrays in resistivity and IP prospecting, Bull. Aust. Soc. Explor. Geophys., 4(1), 1-29.

WHO (1996), Chloride in drinking water, in Guidelines for drinking -water quality, edited, World Health Organization, Geneva. 
Xu, S. (2014), Hydraulic conductivity estimation through complex electrical conductivity in unconsolidated soil., M.S. thesis, Lancaster University, UK.

Y. Lasne, Philippe Paillou, Gilles Ruffi'e, Carlos Serradilla, Francois Demontoux, et al. (2008), Effect of salinity on the dielectric properties of geological materials: Implication for soil moisture detection by means of remote sensing, IEEE Transactions on Geoscience and Remote Sensing, Institute of Electrical and Electronics Engineers, 46 (6), pp.1674-1688.

Zisser, N., A. Kemna, and G. Nover (2010a), Relationship between low-frequency electrical properties and hydraulic permeability of low-permeability sandstones., Geophysics, 75(3), E131-E141.

Zisser, N., A. Kemna, and G. Nover (2010b), Dependence of spectral induced polarization response of sandstone on temperature and its relevance to permeability estimation, Journal of Geophysical Research, 115(B9).

Zong, C. (2002), From deep holes to free planes, Bulletin of the American Mathematical Society, 39(4), 533-555. 